\title{
Pain : attention, emotion, prediction and control
}

Citation for published version (APA):

Arntz, A. R. (1991). Pain : attention, emotion, prediction and control. [Doctoral Thesis, Maastricht University]. Rijksuniversiteit Limburg. https://doi.org/10.26481/dis.19910628aa

Document status and date:

Published: 01/01/1991

DOI:

10.26481/dis.19910628aa

Document Version:

Publisher's PDF, also known as Version of record

\section{Please check the document version of this publication:}

- A submitted manuscript is the version of the article upon submission and before peer-review. There can be important differences between the submitted version and the official published version of record.

People interested in the research are advised to contact the author for the final version of the publication, or visit the DOI to the publisher's website.

- The final author version and the galley proof are versions of the publication after peer review.

- The final published version features the final layout of the paper including the volume, issue and page numbers.

Link to publication

\footnotetext{
General rights rights.

- You may freely distribute the URL identifying the publication in the public portal. please follow below link for the End User Agreement:

www.umlib.nl/taverne-license

Take down policy

If you believe that this document breaches copyright please contact us at:

repository@maastrichtuniversity.nl

providing details and we will investigate your claim.
}

Copyright and moral rights for the publications made accessible in the public portal are retained by the authors and/or other copyright owners and it is a condition of accessing publications that users recognise and abide by the legal requirements associated with these

- Users may download and print one copy of any publication from the public portal for the purpose of private study or research.

- You may not further distribute the material or use it for any profit-making activity or commercial gain

If the publication is distributed under the terms of Article $25 \mathrm{fa}$ of the Dutch Copyright Act, indicated by the "Taverne" license above, 


\section{PAIN:}

ATTENTION, EMOTION, PREDICTION AND CONTROL 


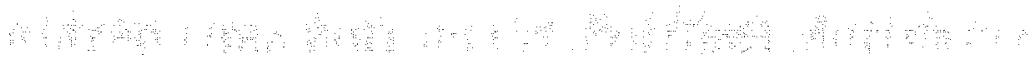




\section{PAIN:}

\section{ATTENTION, EMOTION, PREDICTION AND CONTROL}

\section{PROEFSCHRIFT}

ter verkrijging van de graad van doctor

aan de Rijksuniversiteit Limburg te Maastricht, op gezag van de Rector Magnificus, Prof. Mr. M.J. Cohen, volgens het besluit van het College van Dekanen, in het openbaar te verdedigen op vrijdag 28 juni 1991 om 16.00 uur

door

Arnoud Roger Arntz 
Prof, Dr. M.A, van den Hout

Prof, Dr. SI. Rachman (University of British Columbia, Canada)

\section{Beoordelingscommissie}

Prof. Dr. H.G. Schmidt (voorzitter)

Prof. Dr. W.Th.A.M. Everaerd (Universiteit van Amsterdam)

Prof. Dr, J. Jolles

Prof. Dr. G.J. Kok

Prof. Dr. G. Sartory (University of Wuppertal, Germany)

Lay-out

Isel van Noppen

\section{Druk}

Datawyse Maastricht / Krips Repro Meppel

\section{CIP-DATA KONINKLUJEE BIBLIOTHEEK. DEN HAAG}

Arntz, Arnoud Roger

Pain : attention, emotion, prediction and control / Arnoud

Roger Arntz. - Maastricht: Datawyse. - III.

Thesis Maastricht. - With ref. - With summary in Dutch.

ISBN 90-5291-056-1

NUGI 712

Subject headings: psychology and pain / anxiety and pain / pain : experimental approaches. 


\section{CONTENTS}

Chapter 1. Introduction 7

Dimensions of pain 7

Psychological influences on pain 8

Psychological effects of pain 25

$\begin{array}{ll}\text { Overview of research questions } & 28\end{array}$

Chapter 2. Pain experiences, pain expectations and fear of pain 31

2.1 Generalizability of the match/mismatch model of fear 33

2.2 Is the match/mismatch model based on a statistical 51 artefact?

2.3 Effects of incorrect pain expectations on acquired fear and 59 pain responses

2.4 Predictions of dental pain: the fear of any expected evil, 75 is worse than the fear itself

Chapter 3. Effects of unpredictable increases in pain intensity 91

3.1 The effects of underestimated pain and their relationship to 93 habituation

3.2 The relationship between underpredicted pain and escape 109

3.3 Avoidance of pain of unpredictable intensity 115

3.4 Unpredictable sudden increases in intensity of pain and 121 acquired fear

Chapter 4. Influences of control, attention and emotions on pain 133

4.1 Perceived control and the experience of pain 135

4.2 The interaction between mood and controllability 157

4.3 Attention, not anxiety, influences pain 173

4.4 Attention, anxiety and pain 185

$\begin{array}{lll}\text { Chapter 5. General Discussion } & 195\end{array}$

Psychological influences on pain 195

Psychological effects of painful experiences 202

$\begin{array}{ll}\text { Summary } & 209\end{array}$

$\begin{array}{ll}\text { Samenvatting } & 211\end{array}$

$\begin{array}{lr}\text { References } & 215\end{array}$

List of Abbreviations $\quad 233$

$\begin{array}{lr}\text { Acknowledgements } & 235\end{array}$

$\begin{array}{ll}\text { Curriculum Vitae } & 237\end{array}$ 


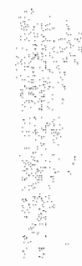

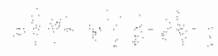

a

$\therefore \quad-\quad \cdots$

a

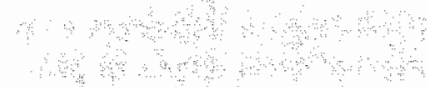

$\therefore \quad \therefore \quad \therefore \quad \cdots$

8
3
3

a : $\quad \because \quad \therefore$ का

ब ब क

$\begin{array}{lll}2 & \\ 4 & \end{array}$ 


\section{CHAPTER 1. \\ INTRODUCTION}

Pain is not merely a physical phenomenon. In a restricted sense, pain refers to a neurological signal indicating that the body has been damaged; it seems therefore to be based on a purely physicall process. However, pain can also be experienced without any discernable injury, and a severe wound may not lead to any pain experience or expression at all (Wall, 1979). In less extreme cases, clear psychological influences can be observed on pain. Thus, there is no one-to-one relationship between physical damage and experiences or expressions of pain.

If there is no direct relationship between tissue damage and pain, then what is pain? The International Association for the Study of Pain (IASP) has defined pain primarily as a psychological phenomenon: 'an unpleasant sensory and emotional experience, associated with actual or potential tissue damage or described in terms of such damage' (IASP, 1979). Various psychological dimensions of pain are distinguished in this definition: perception (sensation), evaluation, emotion and behaviour (description). The relationship with tissue damage is described as being loose. Thus, it is generally accepted that pain is a multidimensional psychological phenomenon, experienced by the subject as if there is tissue damage (which sometimes there is, and sometimes there is not). Therefore, we should expect that pain shows the same sort of plasticity (i.e. the experience of pain and pain responses can be strongly influenced by various psychological factors) as other psychological phenomena, notably the emotions with which it has much in common considering the IASP definition. There are clear neurological reasons to expect such plasticity, since the signal that finally leads to the various pain phenomena can be amplified or reduced at various levels of the nervous system, starting at the spinal level, by higher processes (Wall \& Melzack, 1985).

Within the psychological domain, three factors relating to pain can be discerned: (1) the psychological dimensions of pain itself; (2) the psychological influences on pain; and (3) the psychological effects of pain. In this thesis, research on some aspects of the latter two factors will be reported. The first factor is important, however, and given that pain is not a unidimensional phenomenon, any study on pain should take into account its multidimensionality.

\section{DIMENSIONS OF PAIN}

The multidimensionality of pain refers to the loosely interrelated aspects of pain. Various distinctions have been put forward. The differentiation between the intensity of the experienced stimulation, referring to a more objective, nonevaluative dimension, and the affective aspect of the experience, referring to the evaluative, emotional reactions (pleasure, anxiety, anger, etc.) is well-known (e.g. Gracely, 1979; Price et al., 1980). Others have further differentiated the affective domain into an evaluative and a motivational dimension (e.g., Melzack \& Wall, 1965). Three factor models, well known from the behavioural studies of emotions (Lang, 1985), add overt behaviour and physiological reactions to the cognitive-experiential domain (Vlaeyen et al., 1989). Typical behavioural pain reactions are: withdrawal reflexes, flight, avoidance of stimuli or activities (that are expected to cause or worsen pain), recuperative activities, verbal utterances, grimaces, 
etc. Examples of physiological reactions are: heart rate acceleration, skin conductance responises, vasoconstriction, blood pressure increase, etc. In psychophysiology, three kinds of "reflexes' to acule aversive stimuli, like pain, have been distinguished, each characterized by a distinctive combination of physiological responses: startle, defensive and orienting reflexes (Turpin, 1986). In addition to intensity and affective aspects of the cognitiveexperiential factor, various other types of pain cognitions have been described: e.g. thoughts about causes of the pain, about the development of pain, about the significance of the pain, about the coping possibilities, ete.

The various dimensions of pain are not necessarily strongly interrelated, and therefore each dimension has its own meaning. In most of the experiments reported in this thesis, pain has been measured multidimensionally, without striving for completeness. The emphasis is on subjective experiences of pain and physiological reactions to pain. The subjective experience of pain has been measured by asking the $S$ to rate the experienced painfulness, for instance of an electrically produced aversive stimulation. Most studies did not employ separate intensity and affective/evaluative ratings, because in our laboratory circumstances these dimensions, when separately measured, were found to be strongly interrelated (Arntz \& Lousberg, 1990).

\section{PSYCHOLOGICAL INFLUENCES ON PAIN}

Much research has been done on various psychological influences on pain. In the following, an overview of various psychological factors assumed to influence pain will be presented, resulting in the selection of some factors that will be explored further. Only those factors are presented that are elaborated upon in the present thesis. Thus, a factor such as personallity will not be discussed.

\section{Arousal}

In one of the most important theories of habituation, Groves and Thompson"s (1970) dual-process theory, it is assumed that two processes play a major part in response decrement. Firstly, it is assumed that in the direct neural pathway between stimulus detector and response effector a gradual decline in efficiency of transmission builds up, leading to a decrement in responding. Secondly, a more tonic state of the organism is assumed to influence the general level of responding. This state is not very precisely defined by Groves \& Thompson, but apart from circular definitions (this "state is the general level of excitation, (..) tendency to respond (..) of the organism", p.440), arousal is the most precise definition given by Groves \& Thompson (p.440). The dual-process theory predicts that with high arousal, pain responses are stronger and habituate less. In particular, sensitization (increase in responding) is assumed to be caused by increased arousal.

Without a precise definition of 'arousal' as an independent variable from its effects, it is of course difficult to test the contribution of this factor to the responses to noxious stimuli. However, if we assume that arousal manifests itself in physiological activation, indicators like Skin Conductance Level (SCL) and Heart Rate (HR), and possibly the size of physiological responses like the Skin Conductance Response (SCR), vasoconstriction and the Heart Rate Response (HRR) to painful stimulation, should be related to reduced habituation (see Groves \& Thompson, 1970, p.439 \& 441, for similar operationalization of "state"). The same might be expected from subjective anxiety, which as most would agree, is accompanied by heightened arousal. However, in an experiment in our laborato- 
ry these variables appeared to be unrelated to subjective habituation to pain (Arntz \& Peters, 1990). Moreover, there is no evidence that chronic pain patients, clearly displaying a lack of (subjective) habituation to their own pain, have higher arousal levels as indicated by physiological or subjective indices (Arntz et al., 199/c; Stembach, 1974). This thesis includes an experiment on the influence of anxiety and attention on pain, which is even more relevant for the arousal hypothesis because arousal was experimentally manipulated via manipulating anxiety. It should be noted, however, that the dual-process theory has been developed on the basis of experiments with spinal reflexes. Therefore, the theory might be applicable to habituation and sensitization of spinal reflexes, without being applicable to habituation on higher neurological levels. Eysenck (1967) has differentiated the concept of arousal into physiological activation and cortical arousal. Especially cortical arousal is assumed to be related to pain sensitivity. Although autonomic activation is different from cortical arousal, it is assumed that "Autonomic activation, as in fear, produces higher degrees of (cortical) arousal, and hence lowers sensory thresholds for pain, and also lowers pain tolerance." (Eysenck, 1980, p.308). According to Eysenck, the personality dimension of extraversion-introversion corresponds with cortical arousal, introverts displaying high levels of cortical arousal. It has been reported that this dimension is connected to pain sensitivity, high arousal (introversion) being related to lower pain thresholds and lower tolerance levels (Eysenck, 1980). Thus, like the dual-process theory, Eysencks view predicts that arousal. (and fear) are related to pain sensitivity.

\section{Emotions}

Of the various emotions, anxiety has attracted most attention with respect to its influence on pain. Clinical views often assume that anxiety increases pain impact (e.g., Chapman \& Turner, 1986; Turk \& Fernandez, 1990), as do some theories (e.g., Eysenck, 1980; Furedy \& Doob, 1971a; Melzack, 1973; Melzack \& Wall 1982). However, in some cases it has been observed that anxiety was accompanied with an absence of pain (for instance in severely wounded, scared soldiers; Wail, 1979). There has even been formulated a theory which states that fear and anxiety inhibit pain (Bolles \& Fanselow, 1980). Empirical evidence for either view is inconclusive (see the sections $4.1,4.3 \& 4.4$ for more extensive overviews of these studies and the theoretical views). Therefore, the hypothesis was formulated that a third, hitherto overlooked, factor is responsible for the lack of empirical convergence: attention. This factor might explain why anxiety sometimes is accompanied by increased pain impact (for instance when the $S$ is attending to the pain because of pain related anxiety), and why anxiety is sometimes accompanied by reduced pain impact (when attention is diverted away from pain by anxiety about another stimilus). The three views on the anxiety-pain relationship are put to test in two crucial experiments (see sections $4.3 \& 4.4$ ).

Depressed mood and its counterpart, elation, have received less attention. This is amazing, given the wide domain of psychological processes that is affected by this affective dimension (Clark, 1983). Moreover, depressed mood and affective disorders are very common in chronic pain problems (Romano \& Turner, 1985), leading some authors to hypothesize that chronic pain problems characterized by an absence of underlying physical pathology are instances of masked depression (Blumer \& Heilbronn, 1982). Although there might be more theoretical (and empirical) reasons to assume that chronic, uncontrollable pain leads to depressed affect, for instance via learned helplessness, instead of depression leading to pain (Turk \& Salovey (1984), see also section 4.1), it is interesting to investigate whether depressed mood influences pain. Instead of depression causing pain, 
depressed mood might intensify pain, whereas elated mood might decrease the impact of pain. Only one experimental study addressing this issue was found. In this study the mood of headache patients was influenced by means of the musical mood induction procedure. Elated $S$ s reported less pain than depressed $S$ s. (Martin $e t$ al, 1988). In this thesis one study is included that explores the influence of depressed versus elated mood on pain, in conjunction with the influence of control over pain (see section 4.2).

Even less is known about the influence of anger on pain. On the other hand, it is well known that pain might lead to increased levels of anger, especially when the $S$ fails to find a controlling response (Wall, 1979). For this reason, measurements of angry mood were included in the abovementioned study on the effects of mood and control on pain.

\section{Attention}

There is clear evidence that attention directed to pain results in a larger pain impact than attention distracted from pain (e.g., Blitz \& Dinnerstein, 1971; Bloom et al., 1977; lacono \& Lykken, 1984). Of spontaneously employed pain coping strategies vivid distraction appears to be the most effective (Rosenbaum, 1980). Research comparing several coping strategies indicates that attending to vivid distracting (internal or external) stimuli leads to the most effective pain reductions (Beers \& Karoly, 1979; Grimm \& Kanfer, 1976; Kanfer \& Goldfoot, 1966; Worthington, 1978).

Attention might be an important mediating factor in the effects of other psychological influences on pain. As was hypothesized above, the influence of anxiety on pain might be mediated by attentional factors. In the theoretical section (4.1) on the effects of control on pain it is hypothesized that the pain-reducing effect of controllability might be related to attention. In short, it is argued that when the $S$ can execute control over important aspects of pain, the $S$ is more certain that the pain can be endured without becoming dangerous, intolerable, or neverending. Consequently, the pain becomes less significant for the $S$ and less (unintentional) attention is directed to the pain. Attentional effects might also play an important role in the effects of predictability of occurrence. Miller (1981) has argued that most effects of predictabilitity of occurrence are only found when the $S$ cannot divert his/ her attention (or in some other way 'blunt' the aversive situation). According to Miller, predictable aversive events generally attract more attention than unpredictable aversive events. In contrast, Matthews et al. (1980) have demonstrated that predictable aversive noise is less attended to than unpredictable noise, and that the positive effects of predictability disappear when the $S$ has to attend to the aversive stimulus. Thus, on the one hand warning signals or temporal regularity might attract attention to the coming aversive event, on the other hand, signaled or regularly occurring aversive events might be less surprising, thereby leading to a more superficial processing (Berlyne, 1960).

Representational and cognitive theories also predict that more attention is directed to the pain stimulus when it does not match an internal representation (Chapman, 1980; Sokolov, 1963). Wagner's theory (1981), for instance, assumes that the responses to a stimulus are proportional to the amount of the stimulus representation activated. Since the capacity of activation is limited (cf. the notions of the limited capacity of working memory, Atkinson \& Shiffrin, 1968, and of the 'central processor', Broadbent, 1958, Kahneman, 1973) distractors reduce the amount of stimulus activation and consequently result in smaller responses. Moreover, Wagner's theory states that the responses to a stimulus are reduced proportionally to the amount of stimulus representation which has already been activated by automatic (e.g. conditioning), or perhaps controlled processes, and is in a phase of deactivation. Consequently, the larger the already activated representation, the 
smaller the responses elicited by the stimulus. Thus, "surprising' stimuli (the more surpris" ing a stimulus is, the less its representation is already activated, i.e expected) require more attentional capacity and result in stronger responding and stronger learning.'

In conclusion, attentional factors appear to be very important for the impact of pain, and might play a mediating role in various psychological influences on pain. In some instances, attentional processes may obscure the influence of other factors, a problem frequently encountered in laboratory research with human $S$ s. The clinical relevance of attention seems evident: when pain is not in awareness, it cannot be felt and consequently there is no subjective suffering due to pain. Much of the suffering that is caused by pain is directly related to the claim it lays to attention: when in pain, the $S$ is less free to pay attention to other stimuli.

\section{Operant Conditioning}

The large numbers of patients with chronic pain problems that are apparently unrelated to, or excessive compared to, any underlying physical pathology, have prompted behavjour theorists and therapists to develop conditioning theories to account for these phenomena. Of the operant conceptualizations, Fordyce's is the best-known (Fordyce, 1974). According to this view, pain behaviours can come under control of non-pain stimuli, like any behaviour. Thus, factors like attention from others and avoidance of negatively evaluated situations might reinforce pain behaviour, which might then be displayed in excessive form, given the physical constraints. In addition, healthy behaviour might not receive positive reinforcement, or even criticism. A variable more closely related to pain that might contribute to pain behaviour is the expectation of the patient that healthy behaviours, like physical exercise, work, social activities etc., may worsen the pain problem, and that taking rest and avoidance of certain activities is the best strategy. Such expectations may have been formed on the basis of experiences during the acute phase of pain, but they do not necessarily hold later. Treatment programs for chronic pain based on manipulation of operant factors are well-developed and seem to be especially suited for patients with excessive overt pain behaviour (like avoidance, postures and complaining). The operant theory is silent about the subjective experience of pain: no prediction can be made about the effect of operant conditioning of either pain behaviour or healthy behaviour on the subjective experience of pain. In treatment programs, generally much success is achieved on the behavioural level, but the results on the subjective levels are disappointing (e.g. Linton, 1986; Vlaeyen et al., 1989). The operant views are beyond the scope of this thesis. Nevertheless, some factors that may be related to the acquisition of pain behaviours, notably avoidance behaviour, are treated: pain expectations (chapter 2), experiences with unpredictable pain (chapter $2 \& 3$ ) and learned helplessness (chapter 4).

\section{Classical conditioning}

Three issues can be raised pertaining to the influence of classical conditioning processes on pain. Firstly, can the experience of pain be classically conditioned? Secondly, can re-

\footnotetext{
'For the sake of simplicity, the fact that expectations or anticipatory activation of stimulus representations themselves cause responses is left out of consideration. It should be noted that in case of pain these responses are not pain responses, but anticipatory or conditioned responses. Whether these responses add to the pain responses is discussed under 'predictability".
} 
sponses opposite to pain be conditioned? And thirdly, what is the influence of responses conditioned to waming stimulus on subsequent pain?

The first issue has not received much attention in basic research, but some clinicians claim, and sometimes explain to patients, that pain can be felt without any noxious stimulation because it has becone conditioned (e.g., a dentist claimed that he had observed that some wery frightened patients report pain just before he started drilling (personal communicationy; a self-lielp book for chronic pain patients explains that the experience of pain can be classically conditioned to situational stimuli and activities, Corey, 1989, p.40-41). Most learning theorsts would however seriously doubt the existence of classically condiwoned pain. In a classical paper, Mowrer (1939) defined fear as the conditioned form of the pain reaction. Thus, by definition fear is conditioned to the CS, and not pain. According to the so-called stimulus substitution rule of classical conditioning (Gray, 1975) the CS becomes capable of eliciting (a subset of) the same responses as the UCS. But, the experience of pain is strictly speaking not a response, although it is often treated as such by the experimenter. As has been pointed out by Eysenck (1979), the operational definitions of UCS and UCR as employed by the experimenter should be distinghuished from the phenomenological UCS and UCR in the vilew of the subject. For the experimenter, the objective stimulus is the UCS, and the observed responses (including verbalizations and ratings of scales) are the UCRs. For the subject, however, there is no objective UCS, there is only the experience of the UCS (and sometimes the experience of UCRs): the subject experiences sensations and pain, and possibly bodily reactions (sweating, heart rate acceleration, etc.). Thus, for the subject, the expertence of pain is not an UCR, but the phenomenological UCS. Consequently, the experience of pain cannot be conditioned. The unconditioned responses to pain that are conditioned are, following the nomenclature of Mowrer, fear responses, accompanied by the subjective experiences of fear (apprehension, tension, etc.). It should be noted, however, that some unconditioned responses, like muscle tension or intestinal spasms, when conditioned might themselves cause pain. Thus, some conditioned responses might cause pain, but this does not implicate that the experience of pain has been conditioned (see also Eikelboom \& Stewart (1982) for a theoretical rejection of the possibility that phenomena at the input side of the central nervous system can be conditioned). ${ }^{2}$

In modern views of classical conditioning, it is assumed that the CS becomes capable of eliciting a representation of the UCS, and that the organism reacts to this representation taking into account other momentary factors (e.g. context, information, possibility of avoidance/escape, etc.). On the basis of this modern classical conditioning theory, Chapman \& Gagliardi (1980) raise the question whether somatic conditioned stimuli (CS) might be able to elicit a vivid memory of a painful unconditioned stimulus (UCS), leading to an experience of pain. Thus, the question remains open whether under certain conditions re-

${ }^{2}$ In the past there have been put forward claims that perceptions can be classically conditioned. Most of the studies are poorly documented or have severely methodological shortcomings (see Davies, 1987). More recentlly, Davies (1987) claimed that perceptions can be conditioned. This claim cannot be taken literally because Davies demonstrated that visual after-images can be conditioned. Since an after-image is an experience of a UCR and not a UCS (as is acknowledged by Davies), it is hardly astonishing that it can be conditioned. Thus, this does not imply that perceptions can be conditioned (Paul Eelen is acknowledged for mentioning the studies by Davies). 
presentations of pain might be experienced so vividly, that they lead to an experience of pain. ${ }^{3}$ However, most theorists would object that the representation activated by a CS in its most emotional form would lead to the expectation of a new encounter with the UCS, and consequently lead to fear; or to a memony of pain with reactivation of associated emotional responses. Moreover, to the best of the present authors" knowledge, nobody has ever observed, in controlled circumstances, that CSs are experienced as painful. But, in most laboratory experiments the CS is obviously incapable of eliciting pain (e.g. tones, light signals, slides) ${ }^{4}$. Therefore, a CS should be used that is intrinsically more capable of eliciting pain: the belongingness should be as large as possible. In a pilot study Anja Meijboom and I tried to test this conditioning hypothesis. The control group received 25 stimulif just below pain threshold (produced by means of electrical stimulation at the $S$ 's ankle). The experimental group also received these 25 stimuli, but at the beginning of the series the low intensity stimulus was followed by stimulation of higher intensities, at clearly painful levels a number of times, so that the stimulation at threshold was functionally a CS for the painful stimulation that followed it. We succeeded in inducing elevated subjective fear, but there was no indication of increased subjective pain experiences of the CS or increased physiological responding (figure 1). In this thesis the issue of classically conditioned pain is not explored further. For theoretical and empirical reasons such an enterprise seems unfruitful.

The second issue, the possibility that responses that oppose pain can be conditioned, is much clearer. Classically conditioned responses that counteract pain (responses) have been found: the release of endorphins, morphine-like substances produced by the organism, can be classically conditioned (Bolles \& Fanselow, 1980; Maier et al., 1980; Watkins \& Mayer, 1982). An important factor in the initial release of endorphins is uncontrollability: exposure to escapable shocks does not result in endorphin release, and consequently no conditioning occurs, whereas exposure to unescapable shock does result in conditioning (Dantzer, 1989; Jackson et al., 1979). So far, only contextual cues have been used as CSs. It is unknown whether signals preceding the UCS with a short interval can lead to

\footnotetext{
3In more general terms, Melzack (1973) claims that pain is partly learned - not merely the responses, but the perception of pain. This claim implies that not only the reactions to pain are potentially subjected to learning processes (e.g. operant conditioning, information, modeling), but that the $S$ (in many cases a child) has to learn to perceive what is pain and what is not. A possible classical conditioning pathway for such learning is the experience that certain somatic stimuli are associated with somatic stimuli that already are perceived as painful. See for a test the pillot study discussed in the following.

"There have been reports, however, that painful stimuli can become less aversive by association with strongly appetitive UCSs. Pavlow (1928) for instance, observed that dogs took an electric shock calmly and salivated to it, after it had been paired with food a number of times. At first, the dogs reacted to the shock with howling, barking and struggling to get out of the apparatus. This observation does, however, not imply the reverse, i.e. that a non-painful stimulus can become painful, or a mildly painful stimulus can become intensely painfull. Nevertheless, whereas signalconditioning might not lead to an increased pain experience, evaluative conditioning might. However, evaluative conditioning is supposed to influence the evaluative dimension of a painful stimulus, and probably not its experienced intensity.
} 
temporary endorphin releases. If not, it can be concluded that the designs of predictability studies aiming at testing the pain reducing effects of warning stimuli are incapable of detecting effects of endogenous opioid release (see below). Endogenous analgesia can also be caused by other substances than endorphins (Watkins \& Mayer, 1982), which possibly can also be conditioned to contextual cues (cf. Dantzer, 1989).

The third issue, the influence of a conditioned warning stimulus on responses to the pain stimulus, has received much attention from researchers, albeit under different names (predictability, conditioned diminution, perception, informational control, etc.). This issue is also intrinsically linked to the theory that classically conditioned responses should have a function, namely that of reducing the impact of the aversive UCS. This interpretation of the function of classical conditioning has been the subject of heated debate for more than 20 years, without much progress (cf. Turkkan, 1989).

Besides the functionality theory, there are at least three other conditioning theories that would predict reduced pain impact when the painful stimulus is preceded by a CS. Firstly, the possibility of opposing responses conditioned to associated stimuli has already been mentioned. It should be noted that it is unclear whether pain-opposing responses can be conditioned to warning stimuli preceding the UCS with a short interval. For the time being, it seems safe to limit the class of stimuli capable of eliciting pain-opposing responses like the release of endorphins to contextual stimuli. Secondly, the preparatory set hypothesis (see Miller, 1981) states that conditioned responses (arousal) dampen the impact of the UCS (note the contrast between this theory on the one hand and on the other hand Groves \& Thompson's two-process habituation theory and theories about the alleged pain increasing influence of anxiety). Thirdly, representational theories (Sokolov, 1963; Donegan \& Wagner, 1987; Wagner, 1981) assume that stimuli that are correctly expected are less surprising and are therefore inhibited more. According to Sokolov's theory, the socalled neuronal model of a stimulus growths more rapidly the more the stimulus is predictable (especially regularly occurring stimuli of constant quality). The more a stimulus matches this neuronal model, the more its responses are inhibited. A related, but quite differently stated theory has been developed by Wagner and associates. According to Wagner, the CS automatically leads to responses by bringing the stimulus representation into the deactivation phase (A2). Hence, less stimulus representation is left for activation by the UCS presentation, resulting in less strong responding (in other words, because the stimulus is expected, it is less surprising). The net response depends, however, on the summation of the responses evoked by the CS (during UCS presentation) and the UCS. If the responses evoked by the CS mimick the responses evoked by the UCS, only strong UCSs will lead to weaker UCRs when preceded by a CS; a CS preceding a weak UCS can even lead to stronger UCRs compared to an unsignaled UCS (see Donegan \& Wagner,

\footnotetext{
"See, however, Fanselow \& Bolles (1979) for the endorphin dependency of conditioning of reduction in post-shock freezing of rats to a tone CS preceding the UCS. It is however unclear whether reduction in post shock freezing is an adequate pain reactivity measure (MacLennan et al., 1980). Ross (1986) has demonstrated in 3 experiments that analgesia can be conditioned to a long-lasting CS which starts $30 \mathrm{sec}$ before UCS onset. It is unclear whether this conditioned analgesia was endorphin-mediated, and whether this conditioning is still found with shorter intervals between CS onset and UCS onset. Finally, Fanselow (1979) found that maloxone attennated rats' preference for signaled shock, which suggests that preference for signaled shock is endorphin-mediated and that endorphin-release can be conditioned to the signal of the shock. However, no direct pain-responses were tested.
} 
1987, for a mathematical derivation). When the CS evokes an opposing response, the effect of a CS will always be to diminish the related UCR.
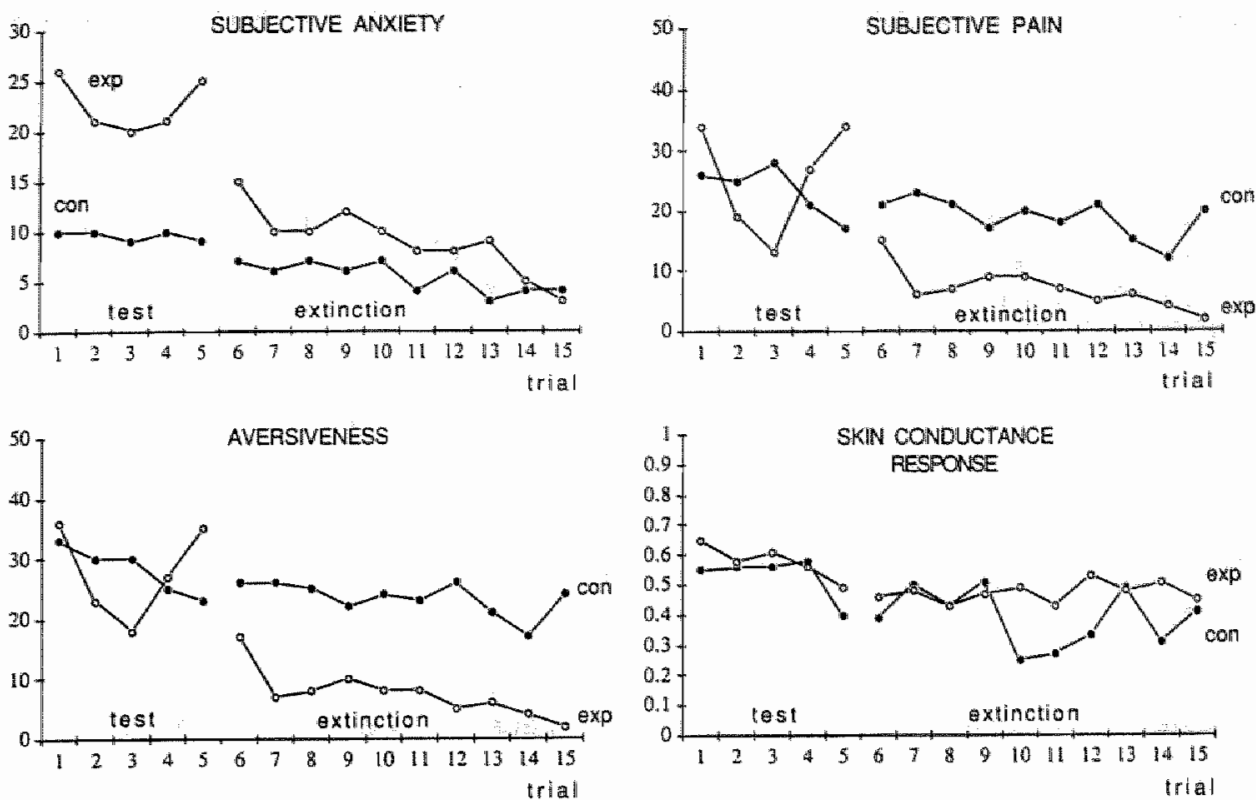

Figure 1. Responses to a stimulus at pain threshold intensity in an experimental $(n=12)$ and in a control group $(n=11)$. During the acquisition phase, the threshold pain stimulus was during 10 trials followed by a very painful stimulus in the experimental group (this strong stimulus was applied to the S's ankle via the same stimulation electrodes at as the threshold stimulus by increasing the intensity level), whereas the control group received unpaired trials only. Five test trials during acquisition are depicted, which were not followed by the strong stimulus (denoted by "test"). During extinction, none of the threshold stimuli was followed by a strong stimulus. During acquisition, subjective anxiety (0-100 Visual Analogue Scale (VAS)) was significantly higher in the experimental group $(F(1,21)=4.63, p<0.05)$ than in the control group, but skin conductance responses (in microSiemens $\left.{ }^{1 / 2}\right)$, subjective pain (0-100 VAS) and subjective aversiveness (0-100 VAS) were n.s. different from the control group $(F s<1)$. During extinction, subjective anxiety in the experimental group quickly diminished to a level comparable to that of the control group $(F(1,21)=1.18, p=0.29)$, SCRs remained comparable $(F<1)$, and subjective pain and aversiveness were even lower in the experimental group than in the control group $(F(1,21)=3.88, p=0.06$; $F(1,21)=4.37, p<0.05)$. These findings do not support the hypothesis that pain at threshold level can be intensified by means of classical conditioning (pairing with a very painful stimulus). On the contrary, a contrast effect seems to describe the results: the threshold stimulus is evaluated as less painful and aversive after having been paired with a more painful stimulus.

Wagner's theory is silent about subjective impact. But, if we assume that activated representations are equivallent to, or at least have high probability to become, the object of awareness, two possibilities follow. First, we can assume that only representations in the so-called active state A1 correspond with awareness. Then it follows that activation of the pain stimulus by conditioned stimuli automatically leads to a reduced subjective pain experience, since CSs are assumed to bring the representation directly into the deactivation A2 
state, which is inaccessible for activation. If, however, it is assumed that representations In the $A 2$ state can also be the object of awareness (which seems unlikely in Wagner"s model; however, it should be noted that Wagner's model has been developed for animal learning processes; note that human $S$ s clearly have the capacity to vividly remember an experience with the help of associated stimuli), the same analysis can be made as above for the other UCRs: only strong pain stimuli are experienced as less intense when signaled.

To summarize, several conditioning theories predict that CSs should be capable to reduce the impact of pain; especially when the pain is intense. Some theories predict that the subjective impact will also be reduced with a $\mathrm{CS}$, others are silent about this issue. The empirical evidence will be presented below, since this issue overlaps for the most part with the issue of predictability.

\section{Predictability}

Various theories have been proposed implying that aversive stimuli, like pain, are experienced as less distressing when their occurrence is predictable (see above; see also Miller, 1981, for a rewiew). Predictabillity of occurrence has been operationalized by presenting a warning signal before the presentation of the UCS vs no warning signal or a randomly occurring signal; by regular vs irregular presentation of the UCS; by immediate vs delayed application of the UCS after a certain point in time; by giving the $S$ s a way to track time until the UCS is presented vs absence of this possibility; or by letting the $S$ s count up to a specified number which coincides with the UCS ws cownting with unrelated presentation of the UCS; etc.

Two ways of testing this prediction have been employed. Firstly, by assessing the preference of $S s$ for predictable $v s$ unpredictable aversive events (either by actual choice or by rating preference (which is sometimes done after the exposure to predictable or unpredictable aversive events, assuming that preference is based upon less distressing impact). Secondly, by measuring subjective impact and physiological responses to the aversive events under predictable and unpredictable conditions. Table 1 presents a summary of the results of various preference studies with human $S$ s that were either found in review articles (Furedy, 1975; Miller, 1981) or in a (limited) search in the literature. It will be clear that most studies have found a preference for signaled aversive events. The studies that failed to find such a preference are mainly from Furedy's laboratory, and there are several reasons why they might divert (e.g. low intensities of shock (just at pain threshold); post exposure ratings; designs that mix predictable and unpredictable UCSs in a unpredictable way; designs that might have been confusing for $S$ s; see also Miller, 1981). Three studies, not included in the table, found that $S$ s prefer unpredictable occurrences of the UCS when a distractor is available (Averill \& Rosen, 1972; Miller (cited in Miller, 1981); Rothbat \& Mellinga, 1972). It should be noted that all these studies involved low-intensity UCSs, thus it remains to be demonstrated which condition $S$ s prefer with really painful stimuli. But, most importantly, the designs were asymmetrical: in the predictable conditions a distractor was not available - which leaves the possibility open that $S$ s prefer predictability when they have the opportunity to use the same coping technique (distracting) with the predictable UCS.

Furedy (1975) has furiously criticized the belief that most $S$ s prefer predictability, but now seems to believe that this notion may be true only for strong stimuli. The reason for post-test measured preference of unpredictable low intensity stimuli might be, as is also suggested by Miller (1981), that under certain circumstances it is more difficult to pay 
little attention to predictable UCSs than to unpredictable UCSs. Most importantly, howey. er, it should be noted that the preference studies cannot answer our question whether predictable pain is less painful than unpredictable pain.

Table 1. Preference for predictable occurrence of aversive events

\begin{tabular}{|c|c|c|}
\hline Badia et al. (1966) & $\begin{array}{l}\exp .1 \\
\exp .2\end{array}$ & $\begin{array}{l}+ \\
+ \\
\end{array}$ \\
\hline Badia et al. (1967) & & + \\
\hline Biederman et al. (1984) & & - \\
\hline Cook \& Barnes (1964) & & + \\
\hline D'Amato \& Gumenik (1960) & & + \\
\hline Elliot (1969) & & + \\
\hline Furedy (1975) & & + (only for high intensity UCS) \\
\hline Furedy \& Doob (1971a) & & $=$ \\
\hline Furedy \& Daob (1971b) & & $=$ \\
\hline Furedy \& Doob (1972) & & $=$ \\
\hline Furedy \& Klajner (1972a) & & + \\
\hline Furedy et al. (1972) & & + \\
\hline Jones et al. (1966) & & + \\
\hline Katz (1984) & & + \\
\hline Lanzetta \& Driscoll (1966) & & + \\
\hline Maltzman \& Wolff (1970) & & + \\
\hline Monat et al. (1972) & & + \\
\hline Perkins et al. (1966) & & + \\
\hline Pervin (1963) & & + \\
\hline
\end{tabular}

+ denotes significantly $(p<0.05)$ more preference for predictability than for unpredicatability.

= denotes NS difference between preference for predictability and for unpredictability.

- denotes significantly $(p<0.05)$ more preference for unpredictability than for predictability.

Only studies with human $5 s$ are included. Various aversive events have been used, mostly shocks and loud tones. Generally three forms of predictability have been employed: a CS preceding the UCS; regular ws irregular occurrence of the UCS; and immediate vs delayed UCS.

The $S$ might prefer predictability for other reasons: the total distress during UCS and nonUCS periods might be less with predictable aversive events, because the $S$ knows he/she is safe when there is no CS, because then there will be no UCS (safety-signal hypothesils, cf. 
Seligman, $\left.1968^{\circ}\right)$; or there may be an intrinsic drive to choose situations which yield the highest amount of information (information seeking hypothesis, cf. Mineka \& Hendersen, 1985). Therefore, impact studies are more suitable for our question.

Table 2 presents an overview of impact studies with human $S s$ which were found in the abovementioned reviews or in our own search. The two most employed impact measures are presented, i.e. subjective intensity ratings and SCRs to the UCS. Subjective and physiological parameters reflecting stress, anxiety etc. during non-UCS periods are not presented. The non UCS-period should be divided into a "safe" period and the anticipation period following the CS (or immediately before the UCS in case of regular UCS presentation). In most research only the anticipation period has been investigated. There is evidence that in the anticipation period arousal is higher with predictable UCSs than with unpredictable UCSs (Miller, 1981), which is of course obvious given the process of conditioning. Considering the possibility that with predictability stress is higher during anticipation, but during safe periods stress is lower, the net result depends on the proportion between safe and anticipation periods (see Epstein, 1973, p.101, for a similar conclusion). Thus, the safety hypothesis would predict that with relatively long safe periods, predictability leads to reduced net stress; but that with relatively short safe periods, the advantages of the safe period may be outweighed by the disadwantage of the anticipatory stress (a related point was made by Miller (1981), but she - implicitly - concluded that with each proportion between safe and anticipation periods the safety hypothesis would predict reduced stress a conclusion that cannot be made in our view). To the best of the present authors knowledge, no study has systematically investigated this factor. It should be noted that most laboratory studies use relatively short intertrial periods, whereas life outside the laboratory is probably more characterized by long periods between important aversive events. In sum, the finding that predictability leads to more stress during the non-UCS period is probably biased by not considering the real safe periods and by designs with short safe periods.

As will be clear from table 2, there is sufficient evidence for the hypothesis that predictable aversive events lead to reduced SCRs. With respect to subjective impact, however, the results are more equivocal. There is a tendency towards reduced impact with predictability, but this might be an artefact of biased publishing (Furedy, 1975). On the other hand, there might be several reasons why some studies failed to find positive impact effects of predictability. Firstly, the intensity of the UCS might have been too low, or not appropriately calibrated to the individual $S$ (e.g. Katz, 1984). Two theories predict that

\footnotetext{
there is evidence that the absence of the warning signal should not be considered as a safety signal, as was originally done in the safety signal theory (Seligman, 1968). Explicit safety signals do not lead to preference of rats for predictability, whereas danger signals do (Fanselow, 1980). Fanselow has proposed an explanation based on learning theory: safety signals may inhibit extinction of fear to contextual cues; whereas danger signals - being explicitly associated with the UCS reduce the association between context cues and the UCS, promoting extinction of fear to contextual cues. Thus, rats are assumed to prefer the situation with the safest context cues. It should be noted that the safety signals in the study of Fanselow were presented only for a small part of the safe period. With safety signals presented during the whole safe period, a simpler explanation is based on the assumption that presentation of a non-noxious stimulus is more easily detected than decontinuation. In other words, it might be so that with an almost permanent safety signal, the organism has to remain vigilant to perceive its absence. Danger signals, on the other hand, are more easily perceived because they are presented instead of stopped, and therefore the organism needs less vigilance to detect them.
} 
with low intensities a decrease of UCS impact will not take place: (1) Wagner's theory (see above) and (2) the notion that warning signals (and counting till UCS delivery, etc.) may direct attention to the UCS: with low intensities it might be easier to direct attention away from unpredictable UCSs (see also Miller, 1981). If an attention-attracting warning. stimulus is presented in both conditions, but in the unpredictable condition there is much more temporal uncertainty about the occutrence of the pain stimulus, predictability results in less pain impact (Katz, 1984; Peeke \& Grings, 1968). It should be noted that this criticism applies to all studies from Furedy's laboratory, which mainly found negative results. Secondly, the subjective pain impact might be reduced by predictability as far as the emotional dimension is concerned. In contrast, objective intensity estimations might be identical in predictable and unpredictable circumstances (see Lykken \& Tellegen (1974) for the same hypothesis). Thus, ratings of aversiveness or pain might be more easily influenced by predictability than objective intensity estimations, a result observed by Price et al. (1980). Since most studies used intensity ratings rather than aversiveness/annoyance/pain ratings, there may be in fact more positive effects of predictability on subjective impact than is suggested in table 2 . Thirdly, there may be an even more specific effect of unpredictable (strong) aversive stimuli that has not received much attention yet: the startle response might be larger with unpredictability. Thus, a specific, hitherto overlooked aspect of the UCS impact might be reduced by predictability. Fourthly, many studies employed within $S$ alternations between (blocks of) warned and unwarned aversive stimuli, or enployed unsignalled changes in UCS intensity, which may have led to such high levels of (perceived) unpredictability that the potential positive effects of the warning signals may have been nullified. It should be noted that predictablility is defined by a strong and reliable CS-UCS relationship (see below for a discussion of the effects of unpredictability of UCS intensity). Fifthly, with low UCS intensities regular presentation of the UCS might be more helpful than a warning signal, presumably because of attentional effects. Warning stimuli might have only positive effects with strong, painful stimuli. Sixthly, the laboratory in which the $S$ s received the aversive stimuli may have functioned as a contextual CS, making the UCS relatively predictable (within the time scope of the laboratory study, which is very small compared to life outside the laboratory). Thus, a more global kind of predictability may have overridden the experimental predictability manipulation (see also the section on classical conditioning, notably the part on endorphins).

In conclusion, despite the large number of negative findings, there is some reason not to reject the hypothesis that predictable pain has less impact than unpredictable pain. This hypothesis may hold for relatively intense pain, for emotional aspects of the pain impact rather than for the judgment of its objective intensity, and when attentional effects are adequately controlled for. Moreover, indirect measurements (performance, reported symptoms, attentional load) suggest that predictability generally leads to less disruption than unpredictability, an effect, however, which may also be attributable to other effects than reduced UCS impact. Given the large number of studies on the subject, and the absence of real progress in the heated discussion about effects of predictability, this thesis does not include any study on predictability of occurrence of pain. The focus is directed to other dimensions of predictability, notably pain intensity (see below). 
Table 2. Impact of averive ewents reduced with predictability?

\begin{tabular}{|c|c|c|c|}
\hline rtudy & subjective impact & $S C R$ & other findings \\
\hline Averill \& Rosenn (1972) & $=$ & & \\
\hline Averill et al, (1977) & - & & 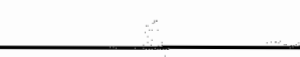 \\
\hline Bowers (1971) & - & & \\
\hline Breitner (1971) & & + & \\
\hline Burger \& Arkin (1980) & $={ }^{4}$ & +8 & $\therefore$ \\
\hline Coles et al (1975) & $=$ & 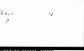 & \\
\hline D'Amato \& Gumenik (1960) & + & & \\
\hline Dimitriivic et al. (1972) & + & & reduced flexor reflex \\
\hline Epstein \& King (Epstein, (1972) & $t^{3}$ & & reduced HRR \\
\hline Epstein \& Roupenian (1970) & & + & reduced HRR \\
\hline Furedy $(1970)^{1,2,5}$ & $=$ & & \\
\hline Furedy $(1973)^{5}$ & $\doteq$ & & \\
\hline Furedy \& Chan (1971) $\quad \begin{array}{l}\exp \cdot 1^{1,2} \\
\exp 2^{1,2}\end{array}$ & $\begin{array}{l}= \\
=\end{array}$ & $\begin{array}{l}= \\
=\end{array}$ & $\begin{array}{l}\text { equal HRR } \\
\text { equal HRR }\end{array}$ \\
\hline Furedy \& Doob $(1971)^{\mathbf{I}}$ & $=$ & $=$ & \\
\hline Furedy \& Doob $(1972)^{2}$ & $=$ & & \\
\hline Furedy \& Ginsberg (1973) & $=$ & . & \\
\hline Furedy \& Klajner (1972b) & $=$ & $=$ & equal HRR \\
\hline Gaebelein et al. (1974) & & + & \\
\hline Geer \& Maisel (1972) & & $=$ & \\
\hline Glass et al. (1969) & $=$ & & \\
\hline Grings \& Schell (1971) & & + & \\
\hline Jones \& Furedy (1989)" & $=$ & a & \\
\hline Katz (1984) & + & + & \\
\hline Kimmel (1966) & & + & \\
\hline Kimmel (1967) & & + & \\
\hline Kimmel \& Pennypacker $(1962)^{2}$ & & + & \\
\hline Klemp \& Rodin $(1976)^{5}$ & $=$ & & \\
\hline Lanzetta \& Driscoll (1966) & & $=$ & \\
\hline Lovibond (1968) & + & + & \\
\hline Lykken (1959) & + & + & \\
\hline Lykken (1968) & & + & \\
\hline Lykken et al. (1972) & $=$ & + & reduced HRR \& P300 \\
\hline Maltzman \& Wolff (1970) & + & + & \\
\hline Merckelbach \& De Jong (1988) & & + & reduced FPV \\
\hline Orlebeke \& van Doornen (1977) & & & increased HRR \\
\hline
\end{tabular}




\begin{tabular}{|l|l|l|l|}
\hline Peeke \& Grings (1968) & & + & \\
\hline Pervin (1963) & + & & \\
\hline Price \& Geer (1972) & & $\begin{array}{l}\text { (=with longer CS } \\
\text { UCS intervals) }\end{array}$ \\
\hline Price et al. (1980) & $+^{3}$ & + & \\
\hline Schell \& Grings (1971) & + & + & reduced HRR \\
\hline Suboski et al. (1972) & & + & \\
\hline Waid (1979) & effect of predictability \\
\hline \hline related findings & less errors on task & $\begin{array}{l}\text { faster reaction times (predictable UCS attracts } \\
\text { less attention) } \\
\text { Finkelman \& Glass (1970) }\end{array}$ \\
\hline Matthews et al. (1980) exp.1 & less physical symptoms \\
\hline Roth (1973) & reduced P300 \\
\hline Sanders (1961) & less variable performance \\
\hline Weidner \& Matthews (1978) & less physical symptoms \\
\hline
\end{tabular}

+ denotes reduced impact with predictable UCS $(p<0.05)$

$=$ denotes equal impact with predictable and unpredictable UCS $(p>0.05)$

- denotes larger impact with predictable UCS $(p<0.05)$

SCR: skin conductance response to the painfull UCS; HRR; heart rate response to the painful UCS; FPV: finger pulse volume response to the painful UCS; P300: specific evoked potential component.

1) UCS intensity varied umpredictably, which may have nullified possible effects of predictability of accurrence.

2) Predictable and unpredictable UCSs were alternated within each $S$ (sometimes in blocks), possibly in a perceived unpredictable manner, which may have resulted in (perceived) noncontingency between CS and UCS.

3) Emotional impact ratings were positively influenced by predictability, intensity ratings were not (e.g. unpleasantness $v s$ intensity).

4) The lack of effect of predictability may be attributed to the presence of a strong distractor in all conditions.

5) Below pain threshold UCS

\section{Other dimensions of predictability and the concept of certainty.}

In contrast to predictability of occurrence, predictability of other pain-related aspects has received less attention. Given the importance that other aspects of pain presumably have, this is, perhaps, amazing. For example, consider dental treatment. Most patients know when to expect pain (e.g. with drilling), but are quite uncertain about how much pain, and for how long, to expect. Pain intensity, duration, locus, and quality are some aspects that are important for the organism, and that are presumably integrated into a mental representation of the pain stimulus (Sokolov, 1963). These relevant aspects may be 
unpredictable for the organism, and to the extent that they are unpredictable, they may lead to a stronger pain impact.

Some research has been done with animals, indicating that (un)predictability of such aspects might be related to pain impact or to preference for information about them. In a review of animal research, Imada \& Nageishi (1982) concluded that rats prefer situations involving certainty to those involving uncertainty about a host of pain related dimensions, such as occurrence, frequency, duration, intensity, quality, pattem, locus, etc.

Even less is known about effects of (un)predictability of these pain aspects in human Ss. Stochastically varying intensity of pain has been observed to be related to an absence of habituation of the flexor reflex (Dimitrijevic et al., 1972). Jones et al. (1966) found that allhough $S$ s asked for information about shock intensity, the number of information requests was much higher for shock occurrence. It should be noted howewer that the proportion of information requests for occurrence ws intensity is probably dependent on the proportion of uncertainties of these aspects. Thus, uncertainty about shock intensity might have been relatively small. Lykken et al. (1972) investigated effects of predictability of locus of pain stimulation (which limb), but found virtually no effect of it, in contrast to the positive effects of predictability of occurrence. Note, however, the relatively low degree of uncertainty with respect to bodily locus in this study. More research has been done on the effects of verbal information about painful stimuli and the responses that are likely to be evoked. The general conclusion from this research is that information reduces pain impact and emotional suffering (see below).

In this thesis effects of unpredictable intensity of pain are explored. Several theories predict that unpredictable intensity is related to a larger impact. For instance theories that assume that the inhibition of the processing of a stimulus is a function of the degree in which it is already mentally represented would consider the modelling of such a representation more difficult for unpredictable varying intensities. Even the two-process theory of habituation would predict reduced habituation. According to Groves and Thompson $(1970)$, for each intensity (partially) different nervous pathways are involved, and because the habituation has to build up in every pathway, this process takes much more time with varying intensity than with constant intensity. Moreover, the arousal would be higher with varying intensity (because of the lack of habituation), which in turn results in stronger responding. However, the two-process theory would not predict reduced responding when the pain intensity is still varying but is made predictable in some other way.

In sum, the investigation of effects of unpredictable intensity on pain impact seems worthwhille for several reasons. Firstly, the intensity dimension has largely been ignored in research on (un)predictability, despite its importance with respect to pain and other aversive events. Secondly, if varying intensity has less impact when it is made predictable, then this finding supports the notion that pain representations at higher levels of the CNS are important with respect to the impact of pain. If however the impact of pain of varying intensity is not influenced by increasing predictability of intensity, the assumption of these influences is redundant and a simpler theory, like Groves and Thompson's two-process theory of habituation, would suffice.

\section{The effects of pain expectations on pain impact.}

So far, the terms 'stimulus representation' and 'expectation' have been used as referring to theoretical constructs, not necessarily corresponding to anything that the human $S$ can report upon. These terms reflect presumed processes within the neuronal system.; which may or may not be related to expectations and stimulus representations that 
are formed by other ways than by direct experience. Thus, it may be possible that on different levels of the central nervous system, (partially) independent stimulus representa tions develop. To what extent do stimulus representations and expectations formed by higher mental processes influence the impact of pain?

The growing interest in cognitive processes has stimulated research exploring the influence of other forms of information than direct experience on pain impact. This research produces strong evidence that human $S$ s can form a pain stimulus representation on the basis of verbally transmitted information, which in turn influences the impact of the pain stimulus. The research of Johnson and coworkers (Johnson, 1973; Johnson et al., 1973) is perhaps the best known in this respect. For instance in the first experiment reported by Johnson (1973) it was found that $S$ s who received accurate descriptions of the sensations experienced during ischemic pain before application of the painful stimulation reacted with less distress to the pain stimulus. This effeet of stimulus information appeared to be independent of subjectively experienced fear, perceived danger or (as assessed in a third replication) anticipated distress. Moreover, it was found that the positive effects of information were especially effective at the start of the pain stimulation, possibly because $S$ s who did not receive the information also formed an accurate stimulus (-sensation) representation by means of their own experience with the stimulus.

Many other laboratory and field studies have been done to assess the effect of information on the impact of painful experiences. Two main dimensions of information have been distinguished: procedural information, that is information about which events will occur; and sensory information, which describes the sensations the $S$ will probably experience. In a meta-analysis of 21 laboratory and field studies on the effects of sensory and procedural information on coping with stressful and painful experiences, Suls \& Wan (1989) concluded that there was clear evidence that sensory preparation is associated with less pain and less distress. Moreover, sensory information appears to have a significantly stronger pain-reducing effect than procedural information. Interestingly, a warning about the extent to which the $S$ can anticipate pain does not lead to weaker pain-reducing effects of the information (as has been hypothesized by Leventhal et al, 1979). Thus, there is ample evidence that the $S$ can form an accurate representation of the pain stimulus (comprising how the stimulus feels) without direct experience with the stimulus, and that this representation reduces pain impact, at least on the emotional and behavioural dimensions.

Whereas the effects of sensory and procedural information on pain have been extensively studied, much less is known about the isolated effects of information about one important dimension of the noxious stimulus: its intensity. Various theoretical predictions can be made. Firstly, accurate information about the intensity might lead to reduced pain impact. Secondly, information that leads to inaccurately hight pain intensity expectations might lead to an ewen more reduced pain impact, either because of the (possible) pain reducing effects of fear, or because of stronger inhibition of the pain stimulus (assuming that the strength of inhibiting influences is correlated with the expected pain intensity, see for instance Wagtuer's theory), or because of the contrast between expectation and experience which might influence the stimulus evaluation. However, it can also be hypothesized that information leading to inaccurately high intensity expectations might lead to an increased pain impact: fear might increase pain, or the final pain impact might be the result of some combination of expectation and experience (as opposed to the contrast between expectation and experience), or increased intensity expectations might direct more attention to the pain stimulus. Similarly, information that leads to inaccurately low pain intensity expectations might lead to increased or reduced pain impact. 
Two relevant experiments were found. Epstein \& Clarke (1970) manipulated $S s^{*}$ expectations about the intensity of a loud noise burst. The results were mixed. The group led to overestimiate the loudness reacted with stronger SCR and HRR to the noise, but with smaller loudness ratings than the group receiving accurate information. The group led to underestimate the loudness reacted with smaller SCRs, higher HRRs and with similar loudness ratings to those of the accurate information group. Thus, there is evidence that two processes influence the effect of intensity information on pain impact: firstly, an immediate effect (reflected by physiological responses) of intensity expectations, and secondly, a later effect which becomes apparent with reassessment of the stimulus (reflected by the subjective ratings), influenced by the contrast between expectation and real stimulus. It should however be noted that the results of Epstein \& Clarke's underestimation group give only partial evidence for this conclusion. This may be related to the limited success of their experimental manipulation in inducing underestimations in this experiment. In a second experiment, Epstein (1971) compared physiological and subjective habituation to two aversive stimuli, electric shock and a noxious sound, ower 5 days. According to Epstein (1971), most people have highly different expectations about a shock (most $S$ s are very concerned) and a sound (most $S$ s are not concerned). In the early period of expsoure self-fulfilling influences of (presented) expectancy were found, whereas in later periods a contrast effect became apparent. However, no direct evidence of the alleged incorrect expectations was reported and the experimental manipulation was done by using two qualitatively different stimuli.

In conclusion, there is clear evidence that stimulus representations formed via higher mental processes can reduce pain impact, and this is especially clear for the formation of sensory impact representations. There is less known about the effects of expectations about the stimulus intensity formed at higher mental levels. It will be clear that this issue is of both practical and theoretical importance. In this thesis this issue is further explored in an experiment in which it is attermpted to lead $S$ s to either overestimate, underestimate or correctly estimate the intensity of a pain stimulus by giving information about the coming pain stimulus.

\section{Controllability}

A long tradition of psychological research has been directed to the issue of whether pain that can be controlled by the $S$ has less impact than uncontrollable pain. This issue is not further treated in this introduction, because a separate section is devoted to it. Suffice to say that a review of the experimental and clinical literature indicates that there is, albeit not unequivocal, evidence that controllability can reduce pain impact. The underlying mechanisms of this effect of controllability are largely unknown. In section 4.1 five theories are discussed. Two possible explanations are rejected: the theory that perceiving control reduces pain impact by reducing anxiety is rejected because it seems unlikely that anxiety increases pain impact and because there have been reports of experiments in which control reduced pain without reducing anxiety; the theory that control reduces pain impact via promoting endogenous opioid release is rejected mainly because uncontrollable pain appears to be related to endogenous opioid release. Three other explanations are proposed: perceiving control changes the meaning of pain; perceiving control is a positive experience per se which can compensate negative experiences; and perceiving control allows people to distract their attention from pain by increasing certainty.

The second of the remaining explanations is further explored in an experiment on the interaction between mood and controllability. If perceiving control is a positive experience 
per se, this might be evident from emotional indices, in particular on the elation-depression dimension. Furthermore, if the presumed positive subjective state as produced by perceiving control is a positive mood state, such a state, induced by other ways than by controlling pain, should also be capable of reducing pain impact: In section 4.2 an experiment is reported in which the interaction between induced mood state (elated ws depressed) and executing control vs failing to execute control over pain is investigated. If control influences pain by inducing a positive mood state, additive effects of mood and control should be observed.

The positive effects of control have been assumed to be mediated by perceptions of control, that is, not actual control but the perception of control is assumed to determine the effects. It has been found that the perception of control, as measured after the task, is influenced by the mood of the $S$ : positive mood is related to higher, even overestinated, perceptions of control, whereas negative mood is related to lower, often accurate, perceptions of control. This research (most has been done by Alloy and coworkers) did not employ aversive stimuli. Moreover, it did not test whether the perceptions of control as influenced by mood resulted in differential effects of control on pain impact. Thus, it might be so that elated mood leads to higher perceptions of control, and that these increased control perceptions lead to a smaller pain impact compared to the lower control perceptions that might be caused by depressed mood. This hypothesis is also put to the test in section 4.2 .

\section{PSYCHOLOGICAL EFFECTS OF PAIN}

Several after-effects of painful experiences can be distinguished. Some effects manifest themselves during anticipation of a following painful experience: subjective-emotional and physiological responses, avoidance behaviour, pain expectations, and efforts to acquire control over the pain stimulus or to cope with the pain. Other effects may be less restricted to anticipatory periods: emotional effects, learned helplessness and memories of pain.

\section{Pain expectations and memories of pain}

In cognitive and cognitive-learning theories expectations play an important role. The expectation of an aversive stimulus is assumed to be a major factor in emotional and behavioural reactions (Alloy \& Tabachnik, 1984; Davey, 1989; Gray, 1975; Beck et al., 1985). In cognitive theories of neurotic disorders it is often assumed that the inappropriately strong affective reactions are based upon inaccurately negative (catastrophic) expectations (Beck et al., 1985; Butler \& Mathews, 1983). In addition, the study of pain expectations might be of interest in itself, as expectations form an important subject in human consciousness. It seems obvious that expectations are influenced by experiences, but often they seem to be less influenced by experiences than could have been expected (e.g., Nisbett \& Ross, 1980). In neurotic problems for instance, overestimation often persists in spite of disconfirmatory experiences (Kent, 1986; Rachman \& Bichard, 1988). In this thesis the hypothesis is explored whether the process of change in expectations of pain is asymmetrical: are pain expectations more easily increased after an underestimated experience than decreased after an overestimated experience? Our investigation of pain expectations is limited to expectations of intensity and aversiveness of the painful experience, although other dimensions can be distinguished (duration for instance). 
The starting point for studying the change process of pain expectations is Rachman's match/mismatch model (Rachman \& Levitt, 1985; Rachman \& Lopatka, 1986a). It states that inaccurately high anxiety or pain predictions ${ }^{?}$ are followed by reductions in the prediction of the next encounter, that accurate predictions are followed by no change in the prediction level, and that inaccurately low predictions are followed by increases in predictions. In several studies it is explored whether this model holds for pain predictions, and various statistical ways to test the model are used. The main interest is however on the deviations from the model: the possible asymmetry between underpredicted and overpredicted experiences in influencing subsequent predictions and fear; and the assumed persistence of overestimation in neurotic problems.

The study of predictions of pain might profit from Alloy \& Tabachnik's (1984) work on covariation assessment by humans and animals. According to these authors, the estimation of covariation the organism makes depends on two sources: the current situational information, and the organisms prior expectations (for instance based on prior experiences). Similarly, expectations about intensity of an aversive event may be based on prior expectations and on current information. In the laboratory, prior expectations are in most cases weak and the hypothesis follows that most $S s$ base their pain predictions on current experiences. Outside the laboratory, prior expectations might be strong, and current information might be less capable of influencing expectations. Rachman and Eyrl (1989) found that women suffering from menstrual pain reduced their prediction of pain over their period, even after an underestimated painful day. The authors conclude that the women use a 'superordinate predictive pattern': based on experience, they expect that pain diminishes towards the end of their period. Similarly, pain expected during dental treatment may be based more upon prior expectations than on the pain that can be expected from the (modern) treatment at hand (Lindsay, 1983; Kent, 1984). Cognitive theorists have stressed that strongly believed ideas are often maintained despite disconfirmatory information (e.g. Nisbett \& Ross, 1980). One of the aims of the study on pain predictions of dental trealment (section 2.3) is to test this hypothesis. A distinction is made between short- and long-term changes in pain predictions. It might be so that current situational information has only a temporary effect, but that in the long run the original prior expectations dominate. It is also hypothesized that memories of pain are similarly influenced by these strong original expectations: in the long run, memories of pain due to dental treatment are assumed to be influenced more by the prior expectations than by the experience proper (Kent, 1985; section 2.4).

In addition to the influence of strong prior expectations on the processing of current information, other factors might be important. Anxiety is a candidate: it has been found that anxiety can disturb attentional and other cognitive processes, which leads to the hypothesis that anxious $S$ s poorly process new information (Wachtel, 1968). According to Eysencks incubation theory (1968), anxiety may become capable to function as a reinforcer (despite the absence of any external aversive reinforcer), reinforcing the emotional reactions to the CS. The incubation theory is silent about expectations, but it is possible that the expectation of the aversive event is also reinforced by experienced anxiety. Lastly, Beck et al. (1985) have similarly suggested that experienced anxiety might function in a positive feedback loop, strengthening the expectations of the feared aversive events.

\footnotetext{
The term expectation is used for the theoretical construct, the term prediction for the rating of expected pain.
} 
To summarize, pain expectations might be an interesting research object, for inherent reasons and because of their (presumed) importance for other psychological variables. In this thesis change processes of pain expectations and the relationship between fear and pain expectations are explored (chapter 2 ).

\section{Emotional effects and learned helplessness}

It is well-known that painful experiences can lead to fear and/or avoidance of a next encounter. In this thesis two possible influences on fear of pain are investigated. Firstly, it is tested whether there is a separate contribution of the discongruency between expected. pain and experienced pain to changes in fear that can be differentiated from the influence of the experienced pain intensity itself (see also above). Such an influence has been found by Kent \& Warren (1986) in a study on changes in fear of dental treatment. Chapter 2 offers studies on this issue. Secondly, the hypothesis that unpredictability of the intensity of the painful stimulus results in increases in fear is put to several tests in chapter 3 . It has already been argued that unpredictability of pain intensity might be an interesting research topic and that it has received little attention so far (p.21-22). In chapter 3 the hypotheses are tested that unpredictable increases in pain intensity lead to increased fear on subjective, behavioural and physiological levels. Increased fear might result from stronger pain experiences, and not from the unpredictability per se. Therefore, in sections $3.2,3.3$ and 3.4 this attempt is made to control for the effect of objective pain intensity.

The reason for distinguishing between the two influences on fear is that the first pertains to discongruencies between subjective pain predictions and pain experiences, whereas the second pertains to procedural unpredictability. Procedural unpredictability of pain is defined here as an unsignalled deviation of the stimulus' intensity from a regular pattern. The influences of these two factors may or may not be based upon one underlying process.

Whereas brief painful experiences lead to fear of a next experience, long-lasting pain can have quite different emotional consequences. It has been described that longlasting pain leads to deactivation and healing behaviours (Wall, 1979; Bolles \& Fanselow, 1980). Depressed affect and even clinical depression might be related to this deactivation, and seems to be quite common in chronic pain patients (see reviews by Romano \& Turner, 1985; Roy et al., 1984). A possible factor intervening between long-lasting pain and depression might be learned helplessness. According to learned helplessness theory, uncontrollable aversive experiences might have a number of negative consequences, which resemble symptoms which characterize depression (Abramson et al., 1978). Pain that is uncontrollable and which leads to disruption of daily functioning (thus reducing control over daily functioning), might lead to depression via learned helplessness ${ }^{8}$. This hypothesis is further discussed in section 4.1 .

\footnotetext{
'This hypothesis has recently received further support in a field study by Rudy et al. (1988) which was not yet published when section 4.1 was written. In this study it was found that the relationship between depression and pain in chronic pain patients was mediated by perceived life interference and self-control.
} 


\section{OVERVIEW OF RESEARCH QUESTIONS}

The research questions addressed in this thesis can be globally divided into three groups. The first group of research questions pertains to the interrelations between pain experiences, pain expectations and fear of pain (chapter 2); the second group to effects of unpredictability of pain intensity (chapter 3); and the last group to influences of control, attention and emotions on pain (chapter 4). All studies investigated repeated pain experiences, allowing for studying change processes over time.

Chapter 2 is devoted to the relationships between pain experiences, pain expectations, and fear of pain. Two main issues are addressed:

(1) in what way do incorrectly predicted pain experiences influence pain expectations and fear of pain?

(2) what is the relationship between pain expectations and fear of pain?

In section 2.1 the influence of incorrectly predicted pain experiences, so-called mismatches, on pain expectations and on fear of pain is explored by investigating the effects of spontaneous mismatches and the effects of an experimentally induced mismatch. Pain expectations are measured by letting $S s$ rate expected pain intensity (pain prediction, a point estimate) and by letting $S$ s rate their subjective certainty about these estimates. Fear of pain is measured on two dimensions: subjectively by means of ratings and behiaviourly by measuring slowing (avoidance) or quickening (escape from the experiment) of $S s^{\prime}$ indications to be ready for the next trial. It is hypothesized that underpredicted pain results in increased pain predictions and in increased fear of pain, whereas overpredicted pain is hypothesized to result in decreased pain predictions and in decreased fear of pain.

In section 2.2 it is questioned whether Rachman's match/mismatch model as applied to spontaneous mismatches reflects 'true' psychological processes or is based on a statistical artefact. More stringent statistical tests are employed to test the model.

In section 2.3 methodological criticism on previous experiments (notably sections 2.1 \& 3.1) on incorrect pain predictions is discussed. A more stringent experimental procedure is used to investigate the effects of incorrect pain predictions on fear of pain, pain expectations, certainty, physiological responding and habituation.

In section 2.4 the relationships between pain experiences, pain predictions and fear of pain are investigated in a clinical setting: during two successive dental treatment sessions. This study aims at (1) validating laboratory findings on the interrelations between these variables in a clinical setting; (2) testing the hypothesis that fearful (phobic) patients are characterized by a chronic tendency to overestimate pain due to dental treatment; (3) investigating change processes in this tendency and in fear of dental treatment; (4) clarifying the relationship between pain predictions and pain memories.

Chapter 3 is devoted to effects of unexpected changes in pain intensity. In the studies reported in this chapter, the pain intensity is objectively increased at one or several trials without the $S$ 's knowledge. Effects of these unpredicted increases in pain intensity on habituation, fear of pain and pain predictions are investigated. The general theory that is tested is that unpredictability of pain intensity has a number of negative effects, notably on fear of pain and on pain predictions.

In section 3.1 the differential effects of an early $v s$ a late unpredicted increase in pain intensity on physiological and subjective habituation, fear of pain and pain predictions are tested. It is hypothesized that a late heightened pain stimulus has relatively larger effects 
than an early heightened stimulus, because the stimulus representation is less developed in the early phase of the sequence.

In section 3.2 the hypothesis is tested that unexpectedly increased pain is related to escape behaviour, controlling for the level of objective intensity.

In section 3.3 the hypothesis is tested that an unpredicted increase in pain is related to subsequent avoidance behaviour.

In section 3.4 the relationship between unpredictable increases in pain and physiological and subjective indications of fear is further explored. In this study, the hypothesis is tested that subjective fear and physiological reactions to a warning signal of the pain stimulus are more profound in a condition with unpredictable increases in pain intensity.

Chapter 4 addresses the influences of control, attention and emotions on pain.

Section 4.1 offers a review of experimental and clinical research on the effects of perceived control over pain. The question is also addressed whether the suffering of chronic pain patients can be understood as resulting from their being in a state of learned helplessness caused by a lack of control over pain and life in general. Finally, possible mechanisms underlying positive effects of perceiving control over pain are discussed. From this discussion a number of hypotheses follow. Some are relevant for the experimental studies reported in this chapter. The first (executing control over pain might be a positive experience per se which can compensate pain) is further explored in section 4.2. The second (anxiety does not influence pain, but attention does) is addressed in sections 4.3 and 4.4 .

In section 4.2 the interaction between mood and control over pain is investigated. The hypothesis is tested that mood influences perceptions of control, and through these perceptions the effects of (lack of) control on pain impact. An alternative hypothesis is that executing control over pain results in a positive mood state which interacts with mood induced in another way. The resultant mood might influence pain impact. Finally, the hypothesis is tested that executing control increases certainty over how much pain can be expected and decreases pain impact by increasing certainty.

In sections $4.3 \& 4.4$ the influences of anxiety and attention on pain are the matter of interest. The hypothesis is tested that not anxiety, but attention influences pain impact. The lack of convergent results of previous studies on the influences of anxiety on pain may have been caused by the operation of this third factor, attention. 
02

ant 9 


\section{CHAPTER 2. \\ PAIN EXPERIENCES, PAIN EXPECTATIONS AND FEAR OF PAIN}

Chapter 2 present four studies on the relationships between pain experiences, pain expectations and fear of pain. Two main issues are addressed: (1) in what way do incorrectly predicted pain experiences influence pain expectations and fear of pain? (2) what is the relationship between pain expectations and fear of pain?

The role of conscious pain expectations is a central issue in this chapter. These expectations are measured by letting $S$ s rate expected pain intensity (pain prediction, a point estimate) and by letting $S$ s rate their subjective certainty about these estimates. Section 2.3 is the most important section of this chapter, because it discusses the role of conscious pain expectations most critically and offers the most stringent experimental test.

In section 2.1 the influence of incorrectly predicted pain experiences, so-called mismatches, on pain expectations and on fear of pain is explored by investigating the effects of spontaneous mismatches and the effects of an experimentally induced mismatch. Fear of pain is measured on two dimensions: a subjective dimension (VAS ratings) and a behavioural dimension (by measuring slowing (avoidance) or quickening (escape from the experiment) of $S s^{\prime}$ indications to be ready for the next trial). It is hypothesized that underpredicted pain results in increased pain predictions and in increased fear of pain, whereas overpredicted pain is hypothesized to result in decreased pain predictions and in decreased fear of pain.

In section 2.2 it is questioned whether Rachman's match/mismatch model as applied to spontaneous mismatches reflects 'true' psychological processes or is based on a statistical artefact. More stringent statistical tests are employed to test the model.

In section 2.3 methodological criticism on previous experiments (notably sections 2.1 \& 3.1) on incorrect pain predictions is discussed. It is argued that unless pain predictions are experimentally manipulated independently from changes in objective pain intensity, it cannot be concluded that incorrect conscious pain expectations play a causal role in the development of fear and in the disruption of habituation processes of pain responses. An experimental procedure is used to manipulate pain expectations independently from objective pain intensity. The effects of incorrect pain expectations on anticipatory variables (fear of pain, pain expectations, certainty, physiological responding) and pain reactions (subjective and physiological reactions) are studied.

In section 2.4 the relationships between pain experiences, pain predictions and fear are investigated in a clinical setting: during two successive dental treatment sessions. This study aims at (1) validating laboratory findings on the interrelations between these variables in a clinical setting; (2) testing the hypothesis that fearful (phobic) patients are characterized by a chronic tendency to overestimate pain due to treatment; (3) investigating change processes in this tendency and in fear of dental treatment; (4) clarifying the relationships between pain predictions and pain memories, including the issue whether the hypothesized bias in pain predictions of fearful patients can also be observed in pain memories. 


\section{SECTION 2.1. \\ GENERALIZABILITY OF THE MATCH/MISMATCH \\ MODEL OF FEAR ${ }^{1}$}

\section{SUMMARY}

An experiment was carried out with 19 normals to investigate the generalizability of the match/mismatch model of fear towards a general model of human information processing of experiences with aversive stimuli. The subjects received 10 painful electric shocks and rated experienced pain, predicted pain at the next trial, certainty of prediction and fear of shock. The time used to fill out the rating scales was also measured. An experimental mismatch was induced in $10 \mathrm{Ss}$. Since the Rachman et al. studies used an unsatisfactory statistical procedure, alternative analyses were tried. Results of the experimental mismatch and of spontaneous matches/mismatches strongly support the core of the model: underpredictions were followed by increases in predicted pain, overpredictions by decreases. The experimental mismatch showed long-term effects on fear and certainty. After underpredictions, acceleration tendencies were found. The results indicate that the $S \mathrm{~s}$ ' information processing is characterized by a strong recency effect and an asymmetry between the two types of mismatch that is not self-evident. The model seems to offer a powerful paradigm for aversive information processing.

\section{INTRODUCTION}

Unpredictable aversive events can have profoundly disturbing effects by producing motivational and learning deficits and emotional disturbances (Seligman, Maier \& Solomon, 1971; Seligman, 1968; Mineka \& Kihlstrom, 1978; Mineka \& Henderson, 1985). Acknowledging that anxiety and panic often appear to come out of the blue and have therefore not been predicted, especially in agoraphobia and panic disorder, Rachman and Levitt (1985) and Rachman and Lopatka (1986a, b) developed new theoretical notions about panic and anxiety disorders. Central in their ideas is the hypothesized negative effect of the underprediction of the fear that phobics expect to experience in a fear-provoking situation: when the anxiety experienced is higher than expected, a strong anticipatory anxiety will develop, leading to avoidance tendencies. The model states that in spite of the anticipation of a high level of anxiety, the anxiety experienced during the next exposure will not necessarily be as high as expected and habituation will occur. Rachman and co-workers report empirical evidence for their match/mismatch model. Rachman and Levitt (1985) conducted a study with 13 mild claustrophobics. They observed that unexpected panic attacks experienced in a small dark room which the Ss were requested to enter, were related to increased in the fear that was predicted to be experienced in the next trial. The fear that was actually experienced was not influenced by previous, unexpected panic. Disconfirmation of predicted fear by lower experienced fear resulted in decreases of predicted fear. The match/mismatch model of panic was subsequently put to the test by Rachman and Lopatka (1986a). Forty-four snake phobics were asked to approach an innocuous live

'Reprinted with permission from Behaviour Research and Therapy, 26, Arntz, A. \& Hout, van den M. Generalizability of the match/mismatch model of fear, 1988, Pergamon Press PLC. 
snake during 10 trialls of 15 sec. Rachman and Lopatka found that predictions of fear tended to increase after underpredictions, and tended to decrease after overpredictions. Reports of actually experienced fear declined steadily and were not related to the occurrence of mismatches. $\$ s$ learned to predict their fear more accurately after a number of trials. Thus, the match/mismatch model seems to explain the results of the panic study as well as those of the "moderate" fear study.

Finally, Rachman and Lopatka (19866) tested the applicability of Gray"s theory on the Behavioural Inhibition System (BIS) (cf. Gray, 1975, 1976, 1982, 1985), using 40 snake phobics in a design similar to that of their previous study. Findings of the previous study were replicated. In addition, the authors conclude that the effects of (mis)matches on speed and hesitation may indicate that the BIS is only triggered by an underprediction and not by an overprediction.

The stimulating findings of Rachman and co-workers raise the question of generalizability of the model. Does the match/mismatch model apply solely to phobic $S$ s, phobic stimuli and phobic anxiety, or does it reflect a more general way of information processing of experiences with aversive stimuli? Generalization of the model is also of interest concerning avoidance- and escape-behaviour. Rachman (1988) rightly points out that the consequences of fear and panic may be more serious than fear or panic episodes themselves. Avoidance and escape rank among the most probable and disabling results of fear.

Recently, a fear-avoidance model of strategies for coping with pain has been proposed (Lethem, Slade, Troup \& Bently, 1983; Slade, Troup, Lethem \& Bently, 1983). Analogous to avoidance models for fear, this model states that avoidance of pain-provoking activities may lead to chronic pain problems. Thus, also with respect to pain, avoidance and escape are important issues. Therefore, the question arises how matches and mismatches generally affect escape and avoidance from aversive stimuli. The third issue of generalization relates to certainty. It has been reported that certainty of prediction decreases as mismatches between prediction and result occur, and that certainty of prediction influences the effects of match/mismatch (Albersnagel, Arntz \& Gerlsma, 1987). It was decided to investigate not only how matches/mismatches affect the direction of predictions (more aversive or less aversive), but also whether the certainty of the predictions decreases after mismatches.

Furthermore, the Rachman et al. studies give rise to a number of methodological and statistical questions. Firstly, the occurrence of underpredictions, overpredictions and matches was not experimentally controlled, leaving unclear whether the observed effects after underprediction were "truly" the result of the underprediction. Secondly, the hypothesis testing used by Rachman et al. is problematic. For determining whether frequencies of matches, underpredictions and overpredictions, as well as frequencies of changes in fear, differed from chance, Rachman et al. employed $\chi^{2}$ goodness of fit tests ${ }^{2}$. The H0 hypothesis stated equal probabilities of each of the three categories ( $\mathrm{H} 0: \pi_{1}=\pi_{2}=\pi_{3}=$ 0.33) (cf. Rachman, 1987). However, it is not clear why under HO matched defined by the arbitrary $2 \mathrm{~mm}$ criterion would occur in $33 \%$, and why matches defined by another criterion, say $3 \mathrm{~mm}$, would not occur in $33 \%$ of the cases. Stated otherwise, the distribution under $\mathrm{HO}$ over the three categories defined by an arbitrary criterion cannot be known a prion. Lastly, the use of VAS scores gives possibilities for analyses on higher levels,

'Underprediction was defined when the experienced fear VAS score exceeded predicted fear by $>$ $2 \mathrm{~mm}$; overprediction was defined when experienced fear was $<2 \mathrm{~mm}$ under predicted fear; otherwise, a match was concluded. 
such as ANOVA and regression analysis because of the continuous level of the responses.

The aim of the present study is two-fold:

1. to study the generalizability of the match/mismatch model; and

2. to overcome some of the methodological and statistical problems of the Rachman $e t$ al. studies.

In order to achieve the aim, the experiment was conducted with normal $S$ s. An aversive (painful) stimulus was used that could be controlled by the experimenters: electric shock. $S$ s were divided into a control group and an experimental group. In the latter group an experimentally controlled underprediction was induced by increasing the intensity of the shock at one of the trials, without the Ss' knowledge. Criteria for match and mismatch were as follows: differences in VAS score between predicted pain and experienced pain of $>+2 \mathrm{~mm}$ on the scale were defined as underpredictions, of $<-2 \mathrm{~mm}$ as overpredictions and of $|\leq \pm 2 \mathrm{~mm}|$ as matches. In addition to the point predictions of pain, $S \mathrm{~s}$ were requested to rate how certain they felt about their expectation and how fearful they were of the next shock. In the present context, avoidance is hard to operationalize. An attempt was made, however, to measure hesitation and escape tendencies, by registering slowing or quickening of $S s^{\prime}$ indications that they were ready for the next trial. Relationships between match/mismatch and changes in other variables were planned to be analyzed by means of contingency tables using $x^{2}$ statistics. Since in this type of analysis the HO probabilities are defined by the marginals, no arbitrary assumptions about them have to be made.

Statistical analyses based on continuous responses were also used. Hypotheses 1-10 (see below) were based on the match/mismatch model and were analogous to the hypotheses confirmed in the Rachman and Lopatka $(1986 \mathrm{a}, \mathrm{b})$ studies. Two hypotheses concerning certainty of prediction and avoidance/escape were added. The hypotheses were formulated as follows:

\section{Pain and predicted pain}

1. Underpredictions will be followed by increases in predicted pain.

2. Overpredictions will be followed by decreases in predicted pain.

3. Overpredictions will be followed by decreases in reported pain.

4. Predicted pain will not change after a correct match.

5. Reported pain will decrease over trials regardless of the occurrence of correct or incorrect matches.

6. Accuracy of prediction of pain will increase with practice.

\section{Fear of shock}

7. Fear of shock will increase after an underprediction.

8. Fear of shock will decrease after an overprediction.

9. Fear of shock will not change after a correct match.

10. Fear of shock will decrease over trials regardless of the occurrence of correct and incorrect matches.

\section{Certainty}

11. Certainty of prediction will increase after a correct match and decrease after a mismatch. 
Speed

12. Time after shock until the $S$ indicates that he/she is ready for the next trial will change after an underprediction;

a. more slowly: evidence for hesitation with respect to the next trial;

b. more quickly: evidence for escape (tendency to terminate the experiment more quickly).

\section{METHODS}

\section{Subjects}

The Ss were 19 volunteers who participated for money. One was a secretary, 18 were students. The average age was 21.74 years (range $18-34$, median $=21, S D=3.68$ ). There were 12 men and 7 women.

\section{Materials}

Materials used included a personality questionnaire (ABV; De Wilde, 1983) that has four subscales: Neuroticism, Psychosomatic Complaints, Extraversion and Defensiveness. The $S$ s indicated experienced as well as predicted pain on visual analogue scales (VAS), $10 \mathrm{~cm}$ horizontal lines ranging from 0 ("not at all painful") to 100 ("extremely painful"). Certainty of prediction was also measured by means of a VAS ("0, not certain at all" to " 100 , completely certain"), as was fear of the next shock (" 0 , not afraid at all" to " 100 , extremely afraid").

\section{Apparatus}

Electric shocks had a duration of 0.5 seconds and were delivered by an a.c. stimulator approved for use with patients. Shocks were given via a pair of Beckman electrodes, pasted on to the middle finger of the non-dominant hand. The skin of this finger was thoroughly rubbed with Hewlett-Packard Redux creme in order to reduce the electrical resistance of the skin and keep it constant (cf. Tursky, 1974).

\section{Procedure}

Ss were randomly assigned to control group $(n=9)$ or experimental condition $(n=10)$. After introduction to the laboratory and obtaining informed consent, the shock level that was to be used was determined (calibration phase). From $0.0 \mathrm{~mA}$ on, the level was heightened in steps of $5 \mathrm{~mA}$ until the $S$ indicated that the shock became aversive. The $S$ was then asked to rate its painfulness on the VAS. The shock level was used in the experiment. After this, the $S \mathrm{~s}$ were asked to complete the personality questionnaire. After completion $S$ s were given a small booklet containing the VAS. The Ss were instructed to complete four VASs after each shock, rating experienced pain, expected pain of the next shock, certainty of expectation and fear of the next shock. Before the first shock, Ss rated only those scales that concerned the shock that had to come. The Ss were asked to indicate after each trial that they were ready to receive the next shock by saying "yes". The time between the delivery of the shock and the $S s^{\prime}$ indication that they were ready was measured in seconds. The $S s$ were neither given any information about the shock levels and 
the number of shocks they would receive, nor about the time they had to wait between their indication that they were ready and the shock. The booklet contained scales for a series of 12 shocks. Ten unannounced shocks were given after random time of waiting (mean time of waiting $30 \mathrm{sec}$, range $15-45 \mathrm{sec}$ ). $S$ s of the control group were given shocks of the level that was determined in the calibration phase (mean level $=43.56 \mathrm{~mA}$, $\mathrm{SD}=23.11 \mathrm{~mA}) . S \mathrm{~s}$ of the experimental group were also given shocks of the callibration level (mean $=54.50 \mathrm{~mA}, S D=16.41 \mathrm{~mA}$ ), with the exception of the third trial. At this trial, the intensity level was heightened with $50 \%$, using the psychophysiological relationship

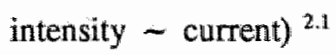

(cf. Lindsay \& Norman, 1977) ${ }^{3}$. From the fourth pain scores, 10 time scores, 11 prediction scores, 11 certainty scores and 11 fear scores were obtained.

\section{RESULTS}

The results will be presented in two parts:

A. the results of the experimentally induced mismatch, and

B. the results concerning matches and mismatches during the whole experiment.

In the first part, the control group and experimental group will be contrasted. In the second part, no separation is made between these groups.

\section{A. The effects of the experimentally induced underprediction}

VAS scores were analyzed by means of MANOVA for repeated measures, testing the changes within each condition as well as comparing the experimental condition and the control group. Two types of pre-test scores were used:

1. scores just before the third shock, and

2. the average of scores at the trials before the third shock.

Hypotheses 1, 5-7, 10-12 will be tested in this part, except for 6 and 12 with one-tailed tests. In other cases, two-tailed tests will be used.

\section{Preliminary: Did the induction succeed?}

The pain-scores of both groups are depicted in figure 1 . After the third shock, $S \mathrm{~s}$ in the experimental group report a mean increase in pain compared to the second shock (10.2 $\mathrm{mm}, t=3.29, p=0.002$, one-tailed), as well as compared to the average first and second shock $(9.6 \mathrm{~mm}, t=3.84, p<0.006$, one-tailed). The control group did not show significant changes in pain scores. The between group effect is significant $(t=2.69, p<0.01$, one-tailed). Thus, the induction succeeded in increasing the subjectively experienced pain.

${ }^{3}$ Note that a $50 \%$ increase in intensity is equivalent to a current that is 1.213 times higher. 
When the criteria mentioned in the introduction ${ }^{4}$ are applied, $8 \mathrm{Ss}$ of the experimental group made underpredictions and two made a match. In the control group, $2 \mathrm{Ss}$ made an underprediction, 2 made at match and 5 made an overprediction (if the matches are excluded and correction for small numbers is used, the groups differ significantly: $\chi^{2}=5.66$, $p<0.02$ ). The differences between predicted and experienced pain for each trial are shown in table 1. As can be seen, the underprediction of the experimental group at trial 3 is significant $(p<0.01$, one-tailed). In sum, the experimental mantpulation succeeded in inducing underpredictions.

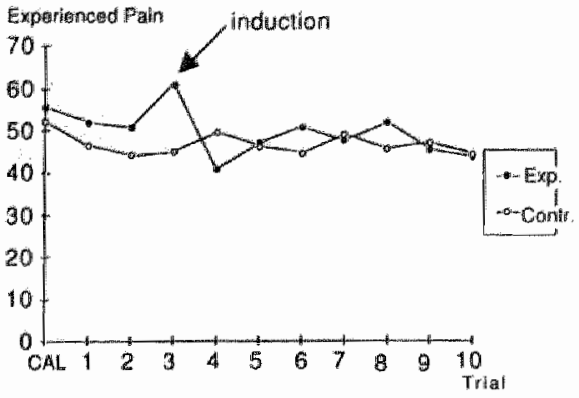

Figure 1. VAS scores of experienced pain for experimental and control groups. CAL indicates the final VAS score at the calibration phase.

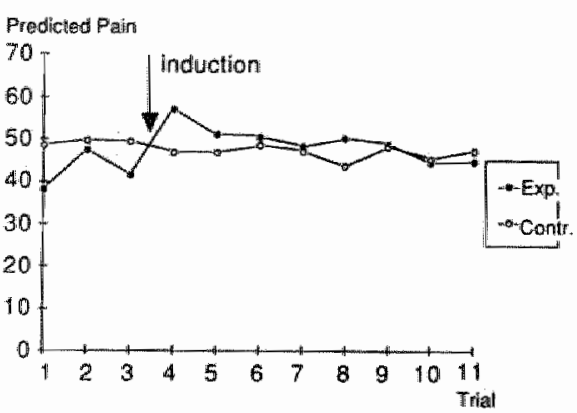

Figure 2. VAS scores of predicted pain for both groups.

\section{Effect on predictions (hypothesis 1)}

All experimental $S$ s showed an increase $(>2)$ in predicted pain after trial 3 . In the control group, $5 S \mathrm{~s}$ showed decreases and 4 increases or no change $\alpha^{2}$ corrected $=8.54$, $p<0.01$ ). The mean predictions at each trial are shown in figure 2 . The between-groups effect of the change from trial 3 to trial 4 was significant $(t=3.34, p=0.002$, one-tailed), as well as the between-groups effect of the change from the average pre-test scores to trial $4(t=2.54, p=0.01$, one-tailed). When the average pre-test predictions were contrasted with the post-test predictions, it was found that in the experimental group prediction 5 was still significantly heightened ( $t=1.75, p<0.05$, one-tailed). From trial 6 onwards, predictions are not longer significantly higher than in the pre-test. To summarize, hypothesis 1 was confirmed: predictions 4 and 5, after the experimentally induced underprediction, were increased. The effect was only temporary.

\section{Long-term effect on pain (hypothesis 5)}

As can be seen in figure 1, the experimental condition shows a rebound effect at trial 4. At this trial, the shock level was reduced to the pre-test level, but $S \$$ reported it as significantly less painful than the pre-test shocks $(t=-2.37, p=0.03)$. Contrasted with the control group, this effect was also significant $(t=-2.30, p<0.04)$. From trial 5 onwards, no significant effects emerged. There was no significant habituation to shocks (trial 9

The criteria are:

underprediction: reported pain - predicted pain $>2$

match:

$-2 \leq$ reported pain - predicted pain $\leq 2$

overprediction:

reported pain - predicted pain $<-2$ 
compared to pre-test: $p=0.44$; trial 10 compared to pretest: $p=0.35$ ). Compared to the calibration pain ratings, pain ratings at trials 9 and 10 were lower to a marginally significant degree (resp. $t=-1.69, p=0.055 ; t=-1.67, p=0.057$; both onemtailed between groups effects were not significant at these trials). Thus, there was no clear evidence for the hypothesized decrease in reported pain (hypothesis 5). However, the experimental induction had no long-term effect on (habituation to) pain.

\section{Long-term effect on matches and mismatches (hypothesis 6)}

As shown in table 1 , the experimental condition shows a mean overprediction at trial 4 $(t=2.03, p=0.073)$ : prediction increased due to the mismatch at trial 3 , but the experienced pain of shock 4 was even lower than at the pre-test. This effect was only temporary: from trial 5 onwards, the experimental condition - as a group - does not show any significant differences between predicted and experienced pain. The number of underpredictions, matches and overpredictions after trial 4 were also analyzed. The groups did not differ significantly $\left(x^{2}=0.33\right.$, df $\left.=2\right)$. In both groups, approx. $34 \%$ were underpredictions, $26 \%$ were matches, and $40 \%$ were overpredictions.

Table 1. Means (and standard deviations) and correlations of predicted and reported pain on a trial-by-trial basis

\begin{tabular}{|c|c|c|c|c|c|c|}
\hline \multicolumn{4}{|c|}{ Control group $(n=9)$} & \multicolumn{3}{|c|}{ Experimental group $(n=10)$} \\
\hline Trial & $\begin{array}{l}\text { Predicted } \\
\text { pain }\end{array}$ & $\begin{array}{l}\text { Reported } \\
\text { pain }\end{array}$ & $r$ & $\begin{array}{l}\text { Predicted } \\
\text { pain }\end{array}$ & $\begin{array}{l}\text { Reported } \\
\text { pain }\end{array}$ & $r$ \\
\hline 1 & $48.56(26.94)$ & $46.56(14.14) 0.25$ & 0.43 & $38.10(13.55)$ & $52.10(12.11)-2.90^{*}$ & 0.30 \\
\hline 2 & $49.78(19.51)$ & $44.22(20.63) 0.93$ & $0.61+$ & $47,40(13,34)$ & $50.80(12.24)-0.64$ & 0.14 \\
\hline 3 & $49.44(21.74)$ & $45.22(12.05) 0.86$ & $0.77^{*}$ & $41.60(15.65)$ & $61.00(11.09)-2.98 *$ & -0.17 \\
\hline 4 & $47.00(20.45)$ & $49.67(17.44)-0.61$ & $0.78^{*}$ & $57.20(18.88)$ & $41.00(17.28) 2.30+$ & 0.03 \\
\hline 5 & $47.00(27.24)$ & $46.44(25.43) 0.14$ & $0.91 * * *$ & $51.10(9.87)$ & $47.10(11.24) \quad 1.51$ & $0.69 *$ \\
\hline 6 & $48.67(29.43)$ & $44.78(25.32) 0.64$ & $0.79 *$ & $50.60(13.99)$ & $50.90(17.52)-0.07$ & $0.59+$ \\
\hline 7 & $47.33(30.25)$ & $49.22(25.44)-0.43$ & $0.90 * * *$ & $48.40(17.62)$ & $47.70(19.51) \quad 0.32$ & $0.93^{* * * *}$ \\
\hline 8 & $43.89(31.79)$ & $45.67(29.75)-0.77$ & $0.98 * * *$ & $50.30(17.53)$ & $51.90(20.59)-0.61$ & $0.92 * * *$ \\
\hline 9 & $48.22(32.38)$ & $47.11(29.12) 0.24$ & $0.91^{* * * *}$ & $49.00(23.65)$ & $42.50(18.61) \quad 1.39$ & $0.78 * *$ \\
\hline 10 & $45.56(32.47)$ & $44.44(30.23) 0.30$ & $0.94 * * *$ & $44.60(20.85)$ & $43.80(19.55)$ & 0.97 事球物 \\
\hline
\end{tabular}

$+p<0.10, p<0.05, * * 0<0.01, * * * 0.001$ (two-tailed)

There is some ewidence that predictions were better at the end of the experiment than at the beginning. Table 1 shows that the average differences between predicted and reported pain tend to decrease. In addition, the correlations between predicted and reported pain increase over the trials. Thus, hypothesis 6 could be confirmed: the accuracy of prediction did increase during the experiment. The experimentally induced underprediction did not have any long-term negative effect on the accuracy.

\section{Effect on fear of shock (hypotheses 7 and 10)}

The mean fear scores of each trial are shown in figure 3. There were no significant between-groups effects.

Within the experimental condition, the change from pre-test to trial 4 was not significant. However, the control group showed significant decreases in fear of shock compared to the pre-test at trials $5,6,8-11$ (all $p^{\prime} s<0.026$ ). Within the experimental condition, only trials 10 and 11 show significantly lower fear of shock scores $(t=-2.46, p<0.03 ; t=$ 


\section{$-2.49, p=0.02)$.}

Hypothesis 10 , which stated that fear of shock would decrease in the long run, regardless of the underpredicted mismatch, was supported: at the end of the experiment both groups show significant decreases in fear. Hypothesis 7, which stated that underprediction would be followed by increases in fear, was not supported. However, the experimental induction seems to have delayed the reduction in fear of shock.

\section{Effects on certainty (hypothesis 11)}

The mean certainty VAS scores for each trial are depicted in figure 4 . There was no significant between-groups effect. Unilike the hypothesis, the experimental condition shows a significant increase in certainty after trial $3(t=2.85, p=0.01)$. The control group also showed a significant increase $(t=2.30, p<0.04)$. The between-groups effect was not significant $(F(1,17)=0.09)$. However, the experimental manipulation seems to have caused a long-term effect: at trials 9-11 the control group shows significant increases in certainty compared to the pre-test $\left(t^{\prime} s>2.30, p^{\prime} s<0.04\right)$, while the experimental group does not show significant changes in certainty at these trials $(p>0.11)$.

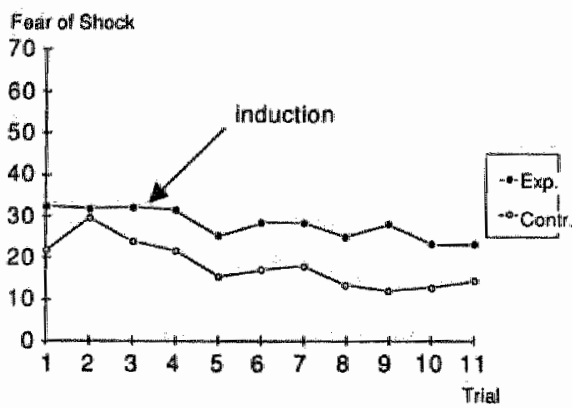

Figure 3. VAS scores of fear of shock for both groups.

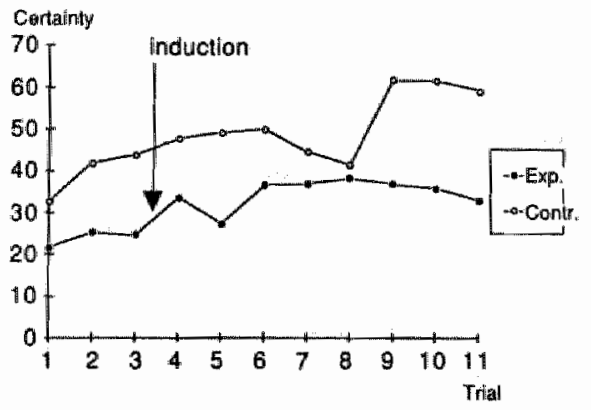

Figure 4. VAS scores of certainty of prediction for both groups.

In sum, hypothesis 11 , which stated that the experimental manipulation would be followed by an immediate decrease in certainty, was contradicted. However, the manipulation seems to have worked against the long-term increase in certainty that $S$ s felt about their predictions: experimental subjects remained more uncertain about their predictions at the last trials.

\section{Effect on avoidance/escape (Mypothesis 12)}

The average time that $S$ s used to complete the scales and to indicate to be ready for the next trial are depicted in figure 5. Both groups show a significant reduction in this time from trial 4 onwards (main effect: $t=-2.44, p<0.03$; between effect: $n . s$. ). From trial 2 to trial 3, the experimental group shows a significant decrease in time $(t=-2.72, p<0.02)$, whereas the control group does not change $(t=0.00, p=1.00)$. However, the between effect is only marginally significant $(t=1.87, p<0.08)$, due to small degrees of freedom and large variances. Contrasting trial 3 with the averaged pre-test times (trials 1 and 2) yields essentially identical results. Thus, there are indications that hypothesis $12 \mathrm{~b}$ can be confirmed: after the experimental induction $S \mathrm{~s}$ show an earlier acceleration in speed. 


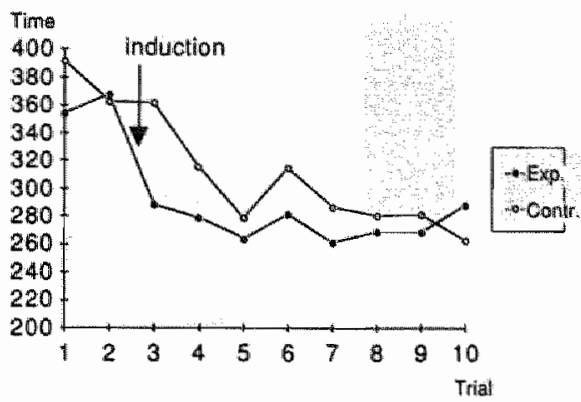

Figure 5. Time (in sec) following shock at Trial $i$ until Ss: indication that they are ready for the next shock.

\section{B. The effects of match and mismatch leaving the experimental manipulation out of consideration}

In this section, the results concerning the effects of match and mismatch will be presented, leaving the differences between the control group and the experimental condition out of consideration. All trials will be analyzed, since it was reasoned that there is no phenomenological difference between an experimentally induced mismatch and a "spontaneous" one. Hypotheses $1-4,7-9,11$ and 12 will be tested in this section.

Effects of match and mismatch on predictions (hypotheses 1,2 and 4)

For each trial the differences (D) between experienced and predicted pain were calculated and classified as underprediction (D>2), overprediction $(\mathrm{D}<-2)$ or match ($2 \leq \mathrm{D} \leq 2)$. Of the $190 \mathrm{D}$ scores, 71 were underpredictions, 47 were matches and 72 were overpredictions. Changes in predictions from this trial to the next trial were also calculated and classified by means of the same criteria. The results of all $S \mathrm{~s}$ and all trials are combined in figure 6. A $\chi^{2}$ test performed on the $3 \times 3$ cross-tabulation yielded a highly significant result $\left(\chi^{2}=84.15, \mathrm{p}<10^{-4}\right)$. As will be clear, underpredictions tend to be followed by increases in predictions $(66.2 \%$ of the underpredictions), overpredictions by decreases (77.8\% of the overpredictions) and matches by minimal changes in predicted pain (34.0\% of the matches) or increases in predicted pain (44.7\%).

For each separate trial, regression analyses were performed with D scores as predictors and change scores in predictions as dependent variables. Figure 7 shows the estimated regression lines. All trials show significantly positive slopes $(r$ 's ranging from 0.43 to 0.86 , $p$ 's ranging from 0.03 to $<10^{-4}$, one-tailed). The average explained variance is $45 \%$. When the regression lines of the 10 trials are averaged, the result suggests that the change in predicted pain may be written as a continuous function of the discrepancy (D) between experienced and predicted pain:

$$
\text { change in expectancy }=0.22+0.57 \times \mathrm{D}
$$

In sum, hypotheses 1,2 en 4 are supported. 


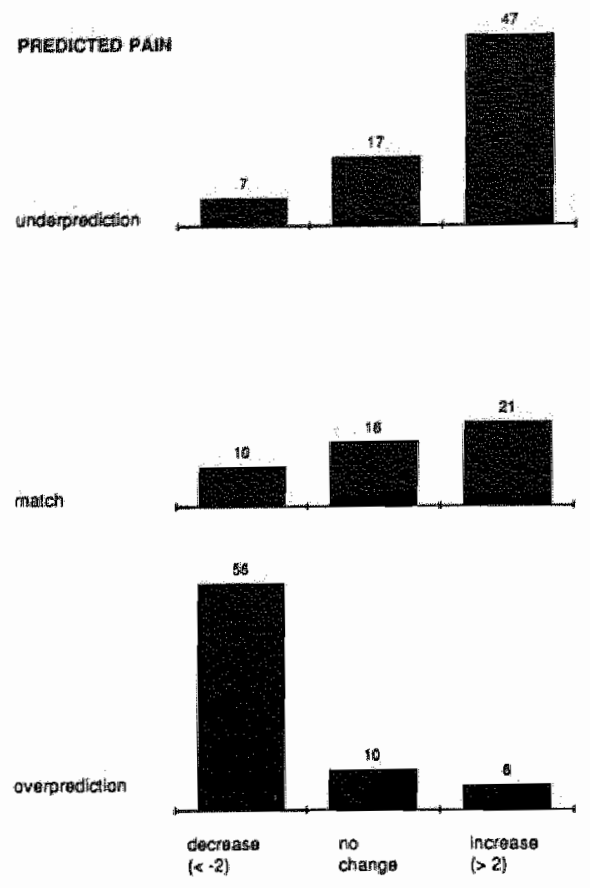

Figure 6 . Number of changes in predicted pain after match/mismatch. Underpredictions were mainly followed by increases in predictions, overpredictions by decreases $\left(p<10^{-4}\right)$.

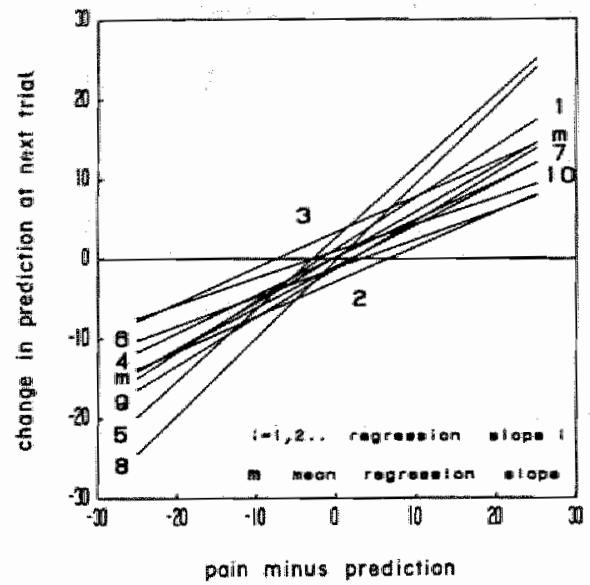

Figure 7. Regression lines of change in predicted pain (vertical axis) as a function of the discrepancy between predicted and reported pain (horizontal axis). Depicted for separate trials and averaged over all trials (M). To the right, underpredictions; to the left, overpredictions.

\section{Effect on subsequent pain (hypothesis 3)}

The relationship between match/mismatch and the number of changes in experienced pain at the next trial is depicted in figure 8 .

As can be seen, $50 \%$ of the underpredictions were followed by a decrease $(<-2)$ in pain at the next trial. After a match, there was also a tendency towards decrease in pain $(45 \%)$. After an overprediction, most of the pain scores were higher $(>2)$ at the next trial $(57 \%)$. The $\chi^{2}$ test yielded a significant result $\left(x^{2}=13.02, p=0.011\right)$. Thus, there was no evidence for hypothesis 3 , which stated that overpredictions would be followed by decreases in pain. In sum, the results seem to reflect a "regression to the mean" effect: after an underpredicted (i.e. a relatively high level of) experienced pain, the next score will be lower; after an overpredicted (i.e. a relatively low level of) experienced pain, the next score will be higher.

Figure 9 illustrates this relationship by means of the regression lines that were estimated in regression analyses of the separate trials. D scores served as predictors, changes in pain scores $\left(\mathrm{P}_{\mathrm{i}+1}-\mathrm{P}_{\mathrm{i}}\right)$ as dependent variables. Five out of nine regressions were significant $(p<0.05)$, as was the regression over all trials $\left(F(1,169)=34.38, p<10^{-4}\right)$. The mean regression slope is clearly negative. 
PAM OF NEXT TRIAL COMPARED TO MATCHMHSWATCH TRIAL
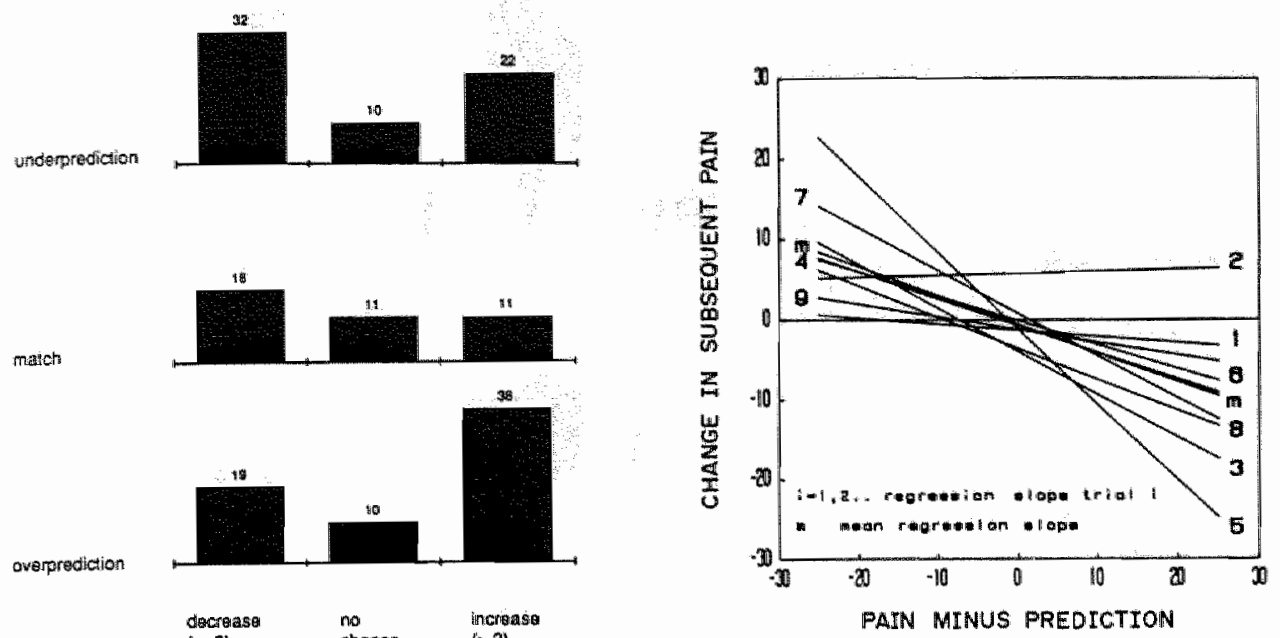

Figure 8. Number of changes in reported pain after match/mismatch. Underpredictions and matches tended to be followed by a reduction in pain level at the next trial. Overpredictions were mainly followed by an increase in pain at the next trial $(p=0.011)$.

Figure 9. Regression lines of changes in experienced pain (vertical axis) following Trial $i$ as function of the discrepancy $(D)$ between experienced and predicted pain (horizontal axis) at Trial i. Depicted for separate trial as well as averaged over all trials (M).

Effect of match and mismatch on fear of shock (hypotheses 7-9)

Figure 10 shows the relationship between match/mismatch and increases in fear (change score $>2)$, no change in fear $(-2 \leq$ change scores $\leq 2)$ and decreases in fear $(<-2)$.

A $\chi^{2}$ test on the $3 \times 3$ contingency table yielded a significant result $\left(x^{2}=11.75, p=\right.$ $0.019)$. After an overprediction, the chance is greater that fear will be reduced $(38.9 \%)$ than after a match $(29.8 \%$ ) or an underprediction $(14.1 \%)$. Of all reductions in fear, $53.8 \%$ was preceded by an overprediction. Increases in fear were not clearly related to match/mismatch. An ANOVA yielded comparable results: overpredictions were followed by a significant mean decrease in fear $(-2.84 \mathrm{~mm}, t=-2.39, p=0.018)$. This change was significantly different from the non significant changes after match and overprediction $(t=$ $-2.05, p=0.042$ ). For each trial regression analyses were performed with $D$ as predictor and fear change as the dependent variable. Only trial 9 showed a significant result $(p<$ 0.05 , one-tailed). When all trials are entered in one regression analysis, a positive relationship of little significance is found $(r=0.19, F(1,188)=6.87, p<0.05$, one-tailed). This result must be interpreted with caution, since scores at different trials of $1 S$ are not strictly independent.

Thus, there is little evidence that there is a linear relationship between change in fear of shock and the discrepancy between experienced and predicted pain. There is no support for hypothesis 7: fear of shock was not increased after underprediction. There is some support for hypothesis 8: there was a reasonable chance that fear was reduced after an 
overprediction. There was also some support for hypothesis 9: after a match, fear did not change substantially in $53.2 \%$ of the cases. However, after each type of mismatch, the chance of no change in fear was highest.

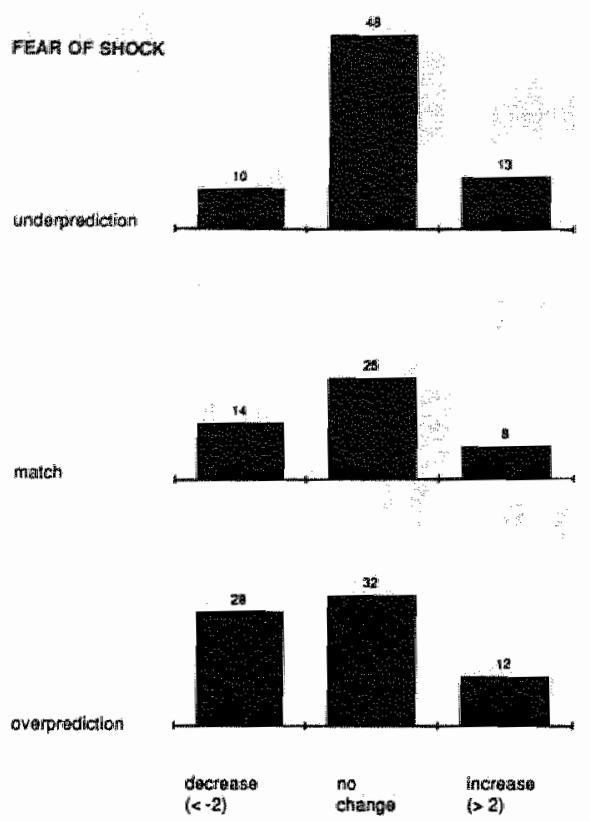

Figure 10. Number of changes in fear of shock after match/mismatch. Fear of shock did not change after most underpredictions and matches. After an overprediction, there were relatively more decreases $(p<0.02)$.

Effect on certainty of predictions (hypothesis 11)

Figure 11 shows the relationship between match/mismatch and the number of increases, decreases or no change in certainty of prediction.

Underpredictions were mainly followed by increases $(43.7 \%)$ or no change $(35.2 \%)$ in certainty. Correct matches were mainly followed by increases in certainty (46.8\%), and overpredictions by decreases $(38.9 \%)$ or no change in certainty $(34.7 \%)$. The $\chi^{2}$ test yielded a marginally significant result $\left(x^{2}=8.84, p<0.07\right)$. Thus, the results gave some evidence for hypothesis 11: certainty tended to increase after a correct match and to decrease after an overprediction. Contrary to the hypothesis, certainty tended to increase or remain stable after an underprediction. These results are also shown by regression analysis. Taking all trials together, changes in certainty were analyzed separately for experienced pain $\leq$ predictions $(D \leq 0)$ and $\geq$ predictions $(D \geq 0)$. For the first group $(\mathrm{D} \leq 0)$ a faint relationship $(r=0.25)$ was found that reflected that stronger overpredictions tended to be followed by larger reductions in certainty $(F(1,95)=6.39, p=0.01)$. For the second group $(\mathrm{D} \geq 0)$, no significant relationship was found $\left(F(1,99)<0.10^{-3}, p=0.99\right)$. As can be seen in figure 12 , matches as well as overpredictions tend to be followed by a small mean increase in certainty of marginal significance, that is to say, of approx. 3.6 $\mathrm{mm}(t=1.70, p=0.09)$. 


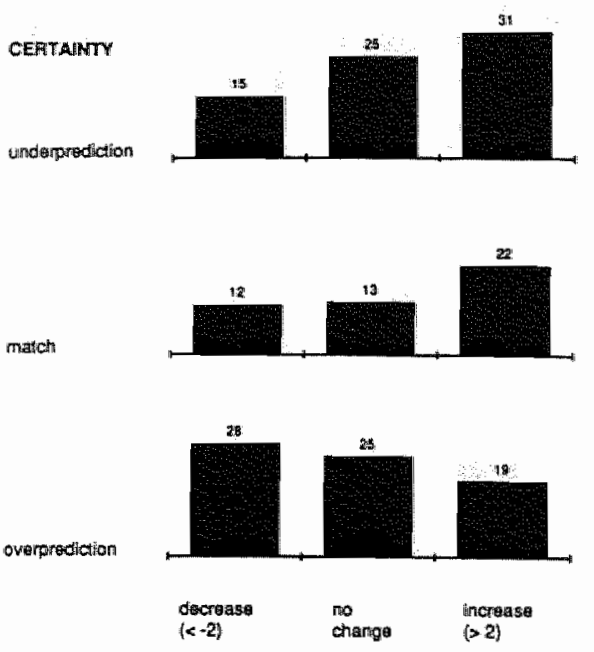

Figure 11. Number of ehanges in certainty after match/mismatch. Certainty of prediction tended to increase after a match and after an underprediction. After an overprediction, certainty tended to decrease. These effects were only marginally significant $(p<0.07)$.

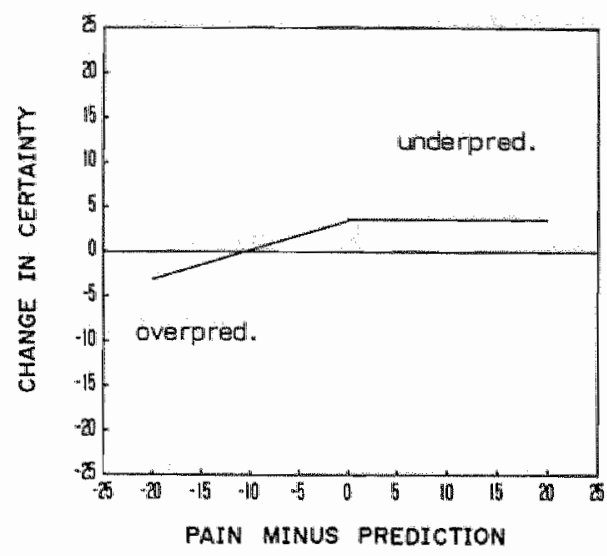

Figure 12. Regression lines of change in certainty (vertical axis) as function of discrepancy $(D)$ between reported and predicted pain. Underpredictions and perfect matches $(D \leq 0$, left-hand line) were analysed separately from overpredictions and perfect matches $(D \geq D$, right-hand line). To the right, underpredictions, followed by a small mean increase in certainty. To the left, overpredictions followed by decreases in certainty proportional to the discrepancy $D$.

\section{Effect on speed (hypothesis 12)}

Changes in time from the shock to the $S \mathrm{~s}$ indication that they were ready for the next shock were analyzed by means of ANOVA. As shown in figure 13, underpredictions were followed by a significant $(p<0.05)$ reduction in time. Matches and overpredictions are not followed by significant changes in time. However, the between effect is not significant ( $F$ $(168,2)=1.21, p=0.30$ ). Analyses of contingency tables and regression analyses yielded non significant results. Thus, there is onlly marginal evidence for hypothesis $12 \mathrm{~b}$, which stated that underpredictions would be followed by reduction in time.

\section{Further investigations}

Factors that might play a role in determining predictions and match/mismatch were investigated. Firstly, the difference between experienced pain and pain experienced at the previous trial was related to the occurrence of match/mismatch. As shown in figure 14, underpredictions were preceded by relatively lower pain scores $(62 \%)$, as were matches $(63 \%)$. Overpredictions, however, were preceded by pain scores that were higher than the pain experienced in the overprediction trial $\left(\chi^{2}=57.73, p<10^{-4}\right)$. 


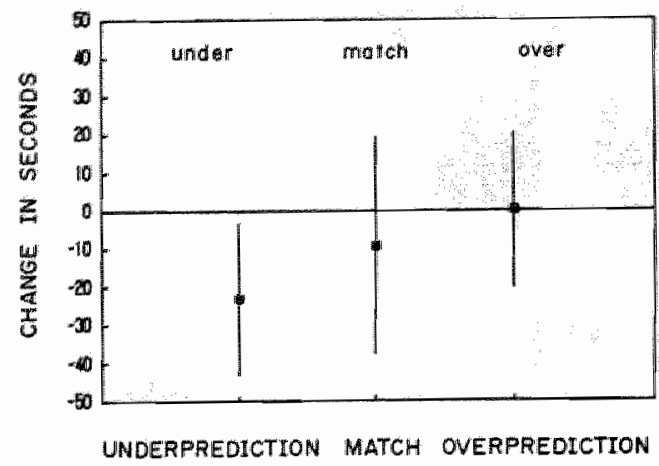

Figure 13. Mean change in time and $95 \%$ confidence intervals after match/mismatch. Underpredictions were followed by a significant mean acceleration $(p<0.05)$.

Regression analysis yielded a strongly linear relationship between the difference in experienced pain at the two trials as predictor $\left(\mathbf{P}_{i}-\mathbf{P}_{i-1}\right)$ and the difference $\mathrm{D}$ between experienced and predicted pain as dependent variable $\left(r=0.60, F(1,169)=93.80, p<10^{-4}\right.$, $\beta=0.71$ ). This result generated the post-hoc hypothesis, that $S s$ might have based their predictions $\left(\mathrm{E}_{\mathrm{i}}\right)$ on the previously experienced pain $\left(\mathrm{P}_{\mathrm{i}-1}\right)^{5}$. This linear relationship $\left(\mathrm{E}_{\mathrm{i}}=\mathrm{P}_{\mathrm{i}}\right.$ 1) was tested for each separate trial by means of regression analysis. The results are shown in figure 15.

The second trial yielded a marginally significant result $(B=0.40, t=1.41, p=0.09$, onetailed). All other trials yielded strongly positive slopes $(\beta=0.62-1.13 ; t=2.69-17.53$; $\left.p=0.01-<10^{-4} ; R=0.55-0.97\right)$. Over all trials, predictions may be estimated by the following linear function:

$$
\mathrm{E}_{\mathrm{i}}=3.89+.92 \mathrm{P}_{\mathrm{i}-1}
$$

This equation explained $65 \%$ of the total variance. Thus, strong evidence was found for the post-hoc hypothesis.

Another investigation was made into the influence of certainty of prediction on the effects of match/mismatch. Certainty scores were correlated with change scores in predicted pain, experienced pain, fear of shock and speed. In case of underprediction, higher certainty scores were related to less increase in predictions $(r=-0.20, p<0.05)$. In the case of overprediction, the opposite relationship was found: higher certainty scores were related to less decrease in prediction $(r=0.18, p<0.07)^{5}$. In case of a correct match, the relationship was zero $(r=-0.02)$. Thus, high certainty of prediction seerns to work against the effect of mismatch on the next prediction. In case of a match, certainty was related to a decrease in experienced pain at the next trial $(r=-0.29, p=0.02)$. In case of mismatch,

This can also be derived mathematically if it is assumed that changes in prediction are linearly related to the discrepancy between experienced and predicted pain: $\mathbb{E}_{\mathrm{i}}-\mathrm{E}_{\mathrm{k}-1}=\mathrm{P}_{\mathrm{k}-1}-\mathrm{E}_{\mathrm{h}-1}$ implies $E_{\mathrm{i}}=\mathrm{P}_{\mathrm{k}-1}$ (see section 2.3).

"Note that after an underprediction, $S \mathrm{~s}$ increased their predictions, and after an overprediction, $S \mathrm{~s}$ decreased their predictions. 
such relationships were absent. Thus, high certainty combined with a match between predicted and experienced pain seems to be related to habituation to the pain stimulus.

Finally, the finding of marginal habituation to the stimulus was investigated further. The mean pain score at calibration and trials 1 and 2 was compared to the mean pain scored at trialls 9 and 10 . Decreases of more than 10 points were assigned to the habituation group $(n=10$, mean change score $-21.00, \mathrm{SD}=6.77)$, other to the non-habituation group ( $n=9$, mean change score $10.65, \mathrm{SD}=13.84$ ). Differences between these groups were investigated. The habituation group did not differ significantly $(p>0.05)$ from the non-habituation group with respect to sex, shock level (mA), VAS pain score at calibra tion and trials 1 and 2, and VAS prediction and certainty scores at trials 1 and 2. Surprisingly, the habituation group showed a significantly higher anxiety score at trials 1 and $2(F(1,17)=4.44, p=0.05)$. The habituation group was also somewhat more neurotic $(F(1,17)=3.91, p<0.07)$, but did not differ from the non-habituators with respect to psychosomatic complaints, extraversion and defensiveness. There was neither a difference between these groups with respect to mismatches at the first two trials, nor with respect to the number of mismatches during the whole experiment $\left(\chi^{2}=3.37, p=0.19\right)$.

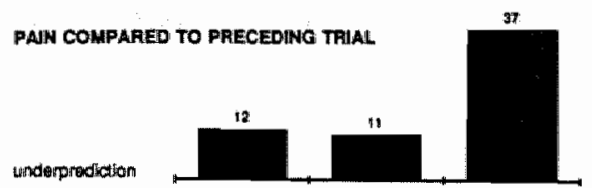

matich

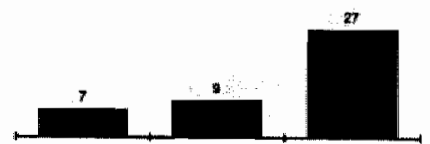

aveiprediction

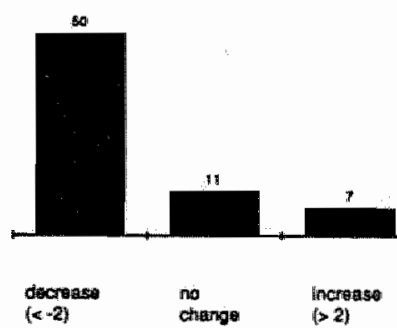

Figure 14. Number of changes in reported at Trial $i$ compared to the preceding trial (Trial i-1). Most underpredictions and matches were based on increases in pain compared to the preceding trial. Most overpredictions were based on pain levels that were lower than at the preceding trial $\left(p<10^{-4}\right)$.

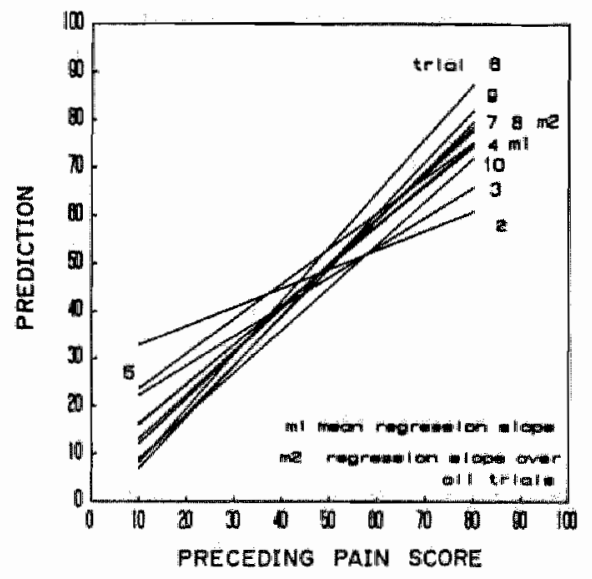

Figure 15. Regression lines of predicted pain at Trial $i$ (vertical axis) as function of experienced pain at Trial $i-1$ (horizontal axis). Depicted for each Trial $t$ as well as averaged over all trials $(\mathrm{M})$. 
Table 2. Summary of hypotheses and results from parts $\mathbf{A}$ and $\mathbf{B}$

Predicted patn

1. Underpredictions will be followed by increases in predicted pain

2 Owerpredictions will be followed by decreases in predicted pain

3. Overpredictions will be followed by decreases in reported pain

4. Predicted pain will not change after correct match

5. Reported pain will decrease over trials regardless of the occurrence of correct or incorrect matches

6. Accuracy of prediction of pain will increase with practice

\section{Fear of shock}

7. Fear of shock will increase after an underprediction

8. Fear of shock will decrease after an overprediction

9.Fear of shock will not change after a correct match

10. Fear of shock will decrease over trials regardless of the occurrence of correct and incorrect matches
A. Results of the experimental underprediction
1. Confirmed
1. Confirmed

2 Not tested

2. Confirmed

3. Not tested

3. Rejected

4.Not tested

5. Rejected. However, the manipulation had no longterm influence on reported pailn

6. Confirmed

4. Confirmed. However, crosstabulation also showed much chance of increase in predicted pain

5. Confirmed by the finding that habituators and non-habituators did not differ with respect to number of match/mismatch 6. Reported at A.
7.Rejected. However, re- 7.Rejected duction in fear was delayed after the experimental underprediction

8.Not tested

9. Not tested

10.Confirmed. However, re- 10. Not tested duction in fear was delayed after the experimental underprediction
8. Confirmed. However, there was considerable chance of no change

9.Confirmed

\section{Certainty of prediction}

11. Certainty of prediction will decrease after a mismatch and increase after a correct match.

11. Subhypothesis concerning decrease after underprediction was rejected. However, experimental Ss were more uncertain at the last trials

12. Time after shock until the $S$ indicates to be ready for the next trial will change after an underprediction
a. more slowly
b. more quickly

12b. Partially confirmed. (within effect significant, between effect marginally sïgnificant)

12a. Rejected
11.Partially confirmed, partially rejected. There was a reasonable chance of decrease in certainty after overprediction. However, underpredictions were mainly followed by increases or no change

12b.Partially confirmed. (within effect significant, between effect n.s.)

12a.Rejected 


\section{DISCUSSION}

Hypotheses and results are summarized in table 2 . On the whole, the match/mismatch model is supported. The core of the model, formulated by hypotheses 1,2 and 4 , is strongly supported. The experimentally induced underprediction was followed by a mean raise in prediction until it (57.2) was almost equal to the mean experienced pain (61.0). The results of the whole experiment also show that the hypothesized relationships (hypothesis 1,2 and 4) are not solely categorical: they are better described by linear functions relating the amount of change in prediction to the magnitude of the mismatch.

Regression analysis showed a strong linear relationship between the amount of change in predicted pain and the discrepancy between predicted and reported pain at the preceding trial. Since the average function (equation 2) almost crosses the origin, it may be concluded that predictions do not change after a correct match. However, cross-tabulation of the categorized differences showed that more matches were followed by increases $(44.7 \%$ ) than by no change $(34.0 \%)$ in predicted pain. On the whole, Ss may have remained guarded by somewhat overestimating the next shock.

Hypotheses 3 and 5 were rejected as far as habituation to the pain stimulus was concerned. No clear mean habituation effects were found. The discrepancy with the Rachman et al. studies may be attributed to differences between their (conditioned) fear stimuli and the (unconditioned) pain stimulus that was used in this study. However, hypothesis 5 was confirmed as far as the effect of match/mismatch concerned: the number of matches/mismatches and the experimental underprediction did not differentiate between $S s$ that habituated and $S$ s that did not habituate or were sensitized to the pain stimulus. Hypothesis 3 was strongly contradicted by the finding that overpredictions tended to be followed by increases in reported pain. Since underpredictions tended to be followed by decreases in pain, the findings may be described as regression to mean: random extreme fluctuations of experienced pain will, in most cases, be followed by a regression of experienced pain to the $S$ 's mean or "true" pain score. Since underpredictions were mainly followed by increases in prediction and overpredictions by decreases, and the next pain score tends to regress in the direction opposite to the change in prediction, the $S s$ created their own mismatches (of the opposite kind) at the next trial. In accordance with this notion was the finding that $S$ s based their prediction almost entirely on the pain experienced at the preceding trial. This relationship (equation 3 ) explained more variance $(65 \%)$ than the relationship of discrepancy between predicted and reported pain with change in prediction $(45 \%)$. The constant (3.89) again shows that Ss tended to be somewhat guarded in their predictions. These findings also suggest that human information processing of aversive stimuli and the generation of predictions is characterized by poor statistics (making no use of regressive tendencies) and a strong recency effect.

They are analogous to the findings in social-psychological and judgemental studies, showing poor human statistical capacities in making inferences (cf. Nisbett \& Ross, 1980). A better strategy from the statistical point of view would be a regressive technique that uses the mean experienced pain score at all preceding trials as prediction.

This recency effect, or not using a "regressive technique", was modulated by certainty of prediction. As in the Albersnagel et al. (1987) study, it was found that certainty of prediction influenced the effects of under- and overprediction on the next prediction: certain $S$ s were somewhat more stable in their predictions, and thus they used more "regressive techniques" in their inferences. It may be interesting to study the influence of a strong experimentally induced mismatch at later trials on the relationship between experienced pain and predicted pain at the next trial. How far does the $S$ 's experiences at earlier trials 
immunixe against the negative effects of such a mismatch? Although, as was discovered, predictions were based on the pain experienced at the previous trial, the accuracy of the predictions increased with practice, and predictions converged with pain scores. Thus, hypothesis 6 was confirmed.

Concerning fear of shock, the most salient deviation from the model that was found, was that after (experimental as well as spontaneous) underpredictions fear scores did not increase. Fear scores tended to remain stable after underpredictions and matches, and even after overpredictions most fear scores decreased only by small amounts. In the long run, fear scores decreased, though.

Underpredictions may, however, have a long-term negative influence on fear: it was found that the experimentally induced underprediction delayed the reduction of fear. The same was discovered about certainty of prediction: experimental underprediction was not followed by a decrease in certainty, but by an increase in certainty (about a higher prediction) and it had a long term negative influence on certainty.

The findings about certainty in the whole experiment suggest an asymmetry between the effects of the two types of mismatch. Underpredictions were mainly followed by increases or no change in certainty, and overpredictions by decreases in certainty, in which the amount of decrease is related to the amount of overprediction.

The two types of mismatch have quite different effects on certainty, indeed. The most puzzling effect is that of underprediction. There is no obvious reason why $S$ s felt more certain about their increased prediction of the next trial after an underpredicted mismatch. It may be theorized, however, that the human organism is inherently alert to underpredicted aversive experiences and weighs them more heavily than overpredicted aversive experiences. Thus, increased predictions based on underpredictions are more strongly believed than decreased predictions based on overpredictions, which raise doubt.

Lastly, we did not find any evidence for slowing/hesitation after an underprediction. The evidence for acceleration was mixed, but both the experimental and the spontaneous underpredictions tended to be followed by increases in speed. Differences from the Rachman et al. studies may be attributed to the different experimental situation. In the Rachman et al. studies $S$ s were asked to approach the stimulus (c.q. initiate and perform own behaviour). In the present study, $S$ s had to receive the shocks passively. By an acceleration Ss could terminate the experimental situation more quickly. Thus, the occurrence of acceleration may indicate the presence of escape tendencies. However, it is, of course, acknowledged that this measure may not be a valid experimental analogue of clinical escape.

On the whole, the results of the experimentally induced underprediction indicate that strong early underpredictions of aversive experiences may have profoundly negative effects: the next experience is expected to be as aversive as the underpredicted one with increased certainty, reduction in fear of the stimulus is delayed, the $S$ s remain more uncertain whether their expectations are right after repeated less aversive exposure. In the present situation this leads to acceleration. In free-choice situations, hesitation/avoidance tendencies will probably dominate. Thus, the experimentally controlled underprediction gave strong support to the generalizability of the match/mismatch model. Results from spontaneous matches and mismatches also gave strong evidence for the generalizability of the model. It seems to offer a powerful paradigm for the processing of information about experiences with aversive stimuli of phobics as well as of non-phobics. 


\section{SECTION 2.2. \\ IS THE MATCH/MISMATCH MODEL \\ BASED ON A STATISTICAL ARTEFACT ?!}

\section{SUMMARY}

In the match/mismatch model, recently formulated by Rachman and coworkers, it is stated that incorrectly predicted aversive experiences are generally followed by an immediate adjustment of the predictions concerning aversiveness of the next experience. This model can be considered to reflect a psychological process of the formation of expectations. In the present article it is argued that a simple HO model, assuming that predictions are completely randomly generated by the subject, may account for the same effects. This $\mathrm{H} 0$ model is used in a stringent test of empirical data to test if there are any effects of the discrepancy between prediction and experience on next prediction that exceed the effects explained by the HO model. Although the HO model produces effects very similar to the empirically observed effects, there is clear support for the hypothesized influence of the discrepancy between prediction and experience. Therefore, the model appears to reflect 'real' psychological processes and not chance findings.

\section{INTRODUCTION}

In 1985 and 1986 Rachman and coworkers published a series of articles on the processing of experiences of fear, emphasizing the part played by predictions of fear (Rachman \& Levitt, 1985; Rachman \& Lopatka, 1986a, 1986b). Central to their ideas are the effects of experiences which are inaccurately predicted. In the laboratory they asked $S$ s to predict how much fear they would experience when confronted with a fear-provoking stimulus. Following exposure to the stimulus, $S \mathrm{~s}$ were asked to rate the amount of fear actually experienced and to make a prediction for the next trial. It was found that overpredictions of fear (defined by predicted fear that is larger than actually experienced) were generally followed by decreases in predictions: $S$ s decreased their fear predictions for the next trial. Following underpredictions (defined as: predicted fear less than experienced fear), $S$ s generally increased their predictions. After a correct prediction (a match) Ss generally did not change their predictions. These findings form the core of the match/mismatch model. The model states that after mismatches (inaccurate predictions) the predictions are immediately corrected (overpredictions are followed by decreases in predictions, underpredictions are followed by increases), whereas matches (adequate predictions) do not lead to changes of prediction.

The model is stated generally, and appears to describe the effects of inaccurate predictions on next expectations not only for fear-provoking stimuli, but for all kinds of stimuli. Further research has indeed shown that the model also holds for pain (Arntz \& van den Hout, 1988; Rachman \& Lopatka, 1988; Arntz et al., 1990a) and for aversive sounds (Lavy et al., 1990). Probably, it also holds for pleasant experiences.

${ }^{1}$ Reprinted with permission from Behaviour Research and Therapy, 28, Arntx, A., Hout van den, M., Lousberg, R. \& Schouten, E. Is the match/mismatch model based on a statistical artefact? 1990, Pergamon Press PLC. 
The central assumption of the model is, that the inaccuracy of a prediction has an immediate influence on the next prediction. Appealing as the idea might be, it is not that easy to test it empirically. Spontaneous predictions and experiences of fear, pain, aversiweness etc. are outside the experimenters' control and may be statistically interrelated in such a way that it is difficult to find the right statistical testing procedure. In an attempt to bypass this problem, Amtz \& van den Hout (1988) tried to experimentally control the mismatch by unexpectedly increasing the level of painful electric shock. It was found that Ss increased their predictions for the next shock immediately, by which the model was supported. In following experiments using this manipulation this finding was replicated (Arntz Lousberg, 1990; Amtz et al., 1990b).

Although the experimentally controlled mismatches led to effects that are predicted by the model, the issue remains whether spontaneous mismatches do lead to 'real' corrections of predictions. Up to now, the attempts to test this hypothesis used statistical procedures (goodness of fit tests, crosstabulation with $x^{2}$ tests) that did not adequately control for possible chance effects (e.g., Rachman \& Lopatka, 1986a; Amtz \& Van den Hout, 1988). To get more insight into the problem we proposed a simple statistical model, which represents the null hypothesis.

\section{THE HO MODEL}

Let us assume that predictions and reports of the experience are independent, that they have the same statistical distribution, and that predictions are randomly generated by the $S$ (that is, they do not reflect any 'real" psychological process: predictions are not influenced by the experience $)^{2}$. Let us assume further - for the sake of simplicity - that $1 / 3$ of the predictions can be classified as 'high' (H), 1/3 as 'medium' (M) and 1/3 as "low' (L); and that the same classification can be made for the experiences. The random combinations of predictions and experiences can be classified as overpredictions $[(H, M),(H, L),(M, L)]$, as matches $[(H, H),(M, M),(L, L)]$, and as underpredictions $[(M, H),(L, H),(L, M)]$. It will be clear that the expected probabilities of these classifications are $1 / 3$ for each $(1 / 3$ is overprediction, etc.). ${ }^{3}$ Since we assumed that predictions are randomly generated, after each type of (mis)match $1 / 3$ of the predictions will be ' $H$ ', $1 / 3$ ' $\mathrm{M}^{\prime}$ ' and $1 / 3$ ' $L$ '. Computation shows that after an overprediction, the chance that the next prediction will be lower than the previous prediction is 5/9: that is, considerably higher than the chance that an overprediction is followed by no change in prediction (1/3) or by an increase (1/9). ${ }^{4} \mathrm{By}$

\footnotetext{
"Whether experiences are randomly generated or have a 'real' basis is not important for the model under $\mathrm{HO}$.

It is interesting to note that these frequencies approximate the empirically observed frequencies very well (see Rachman \& Lopatka, 1986a; Arntz \& van den Hout, 1988). There are, however, exceptions, in that the number of overpredictions is much higher in clinical problems (e.g., Arntz, wan Eck Heijmans 1990).
}

${ }^{4}$ Overpredictions are combinations of $\left(H_{2} M\right),(H, L)$ or $(M, L)$, all with a chance of $1 / 3$. After $(H$, $\mathrm{M}), 1 / 3$ of the predictions will be $\mathrm{H}, 1 / 3 \mathrm{M}$, and $1 / 3 \mathrm{~L}$. The last two predictions are lower than the previous predictions, each with a chance of $1 / 3 \times 1 / 3=1 / 9$. The (prediction-experience-next prediction) sequences $(H, M, M)$ and $(H, M, L)$, as well as $(H, L, M),(H, L, L)$ and $(M, L, L)$ are overpredictions that are followed by decreased prediction. Each has a chance of $1 / 3 \times 1 / 3=1 / 9$. Thus, the chance of decreased prediction after overprediction is the sum of the chances of these five sequences, $5 / 9$. 
the same logic, the chance that after an underprediction the next prediction will be higher is $5 / 9$, etc. These expected frequencies are depicted in figure 1 and are remarkably similar to the patterns that are empirically observed. The expected pattern derived from $\mathrm{HO}$ resembles the observed patterns.

PRAB B

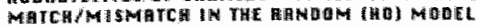

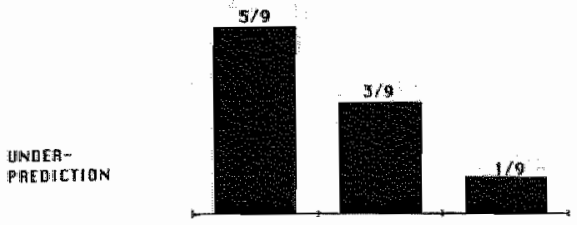

MATEH
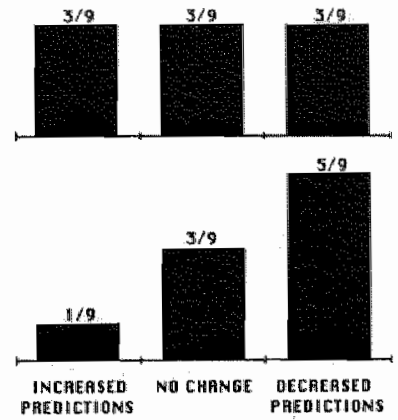

Figure 1. Probabilities of increase, no change, and decrease in prediction after underprediction, match and overprediction in random data (H0 model). The distribution resembles the empirically found distributions very much.

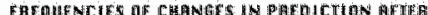

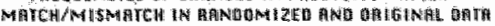

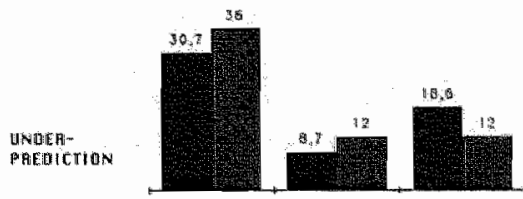

ARTCH

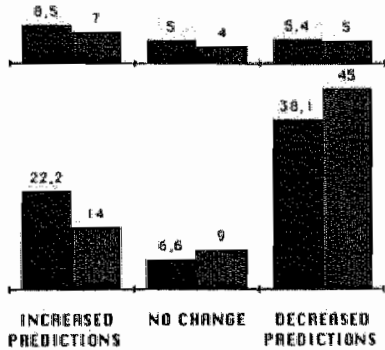

Figure 2. Distributions of changes in prediction of fear after (mis)-matches (1) as found after individually randomizing the predictions of agoraphobic patients (solid bars), and (2) as empirically found in the sample (hatched bars). The numbers of underprediction, overpredictions and matches in the randomized data were held equal to the empirical numbers. Although the randomization produced a distribution much alike the empirical distribution, the empirical findings differ significantly from the random distribution, supporting the match/mismatch mode $\left(x^{2}(4)=12.60, p=0.013\right)$.

This pattern of course reflects the 'regression to the mean effect' that occurs when a measurement is repeated after an extreme outcome as far as there is a random error in measurement: after a high prediction, it is most probable that the experience will be relatively lower (thus, that the prediction will be an overprediction), and this holds of course also for the next prediction, which will also with (the same) high probability be lower. Although the predictions are assumed to be statistically independent ${ }^{5}$, overpredictions and

${ }^{s}$ Generally, the regression to the mean effect occurs proportionally to the independent random error of the observations. Here it is assumed that each observation is a completely randorn "error". 
decreases in predictions are related by the nature of the random process generating the predictions, and the same holds of course for underpredictions and increases in predictions: Therefore, the question arises whether the observed effects of spontaneous mismatches reflect a psychological process or are based on regression to the mean. If there is any psychologically meaningful process underlying changes after mismatches, it must be demonstrated that changes in predictions after mismatches are more strongly influenced by the discrepancy between prediction and experience than can be predicted by a regression to the mean effect alone. In the following, two studies will be described that tried to test the hypothesis that there is such a process. The studies use real data as obtained in a study with agoraphobic patients (Van den Hout et al., 1989) and in a laboratory study on repeated painful experiences (Antz \& Lousberg, 1990).

\section{STUDY 1}

The data in this study were gathered from agoraphobic patients treated by means of exposure in vivo at the local mental health centre. Immediately after each exposure exercise, each patient rated anxiety experienced (on a $100 \mathrm{~mm}$ VAS) during the exposure exercise, and predicted anxiety of the next exercise. There were 15 patients, who all rated the first 10 exposure exercises of their treatment hierarchy. Because of missing data, there were 144 valid observations of predicted anxiety, reported anxiety, and predictions of anxiety for the next exercise. For further details the reader is referred to van den Hout $e t$ al. (1989).

In this study it was assumed that each $S$ generated his/her predictions randomly from his/her own distribution. The effects of mismatches on next predictions under this null hypothesis was tested by taking within each $S$ all possible combinations of two predictions and one experience (complete randomization). A criterion of a $7 \mathrm{~mm} .{ }^{6}$ difference between prediction $(\mathrm{P})$ and experience $(\mathrm{E})$ was used for classifying combinations as underpredictions ( $\mathrm{P}-\mathrm{E}<-7 \mathrm{~mm}$.), overpredictions (P-E $>7 \mathrm{~mm}$.) or matches $(-7 \mathrm{~mm} . \leq \mathrm{P}-\mathrm{E} \leq 7 \mathrm{~mm}$.). The same criterion was used for classifying increases in predictions $\left(\mathrm{P}_{\mathrm{i}}-\mathrm{P}_{\mathrm{i}+1}<-7 \mathrm{~mm}.\right)$, decreases $\left(\mathrm{P}_{\mathrm{i}}-\mathrm{P}_{\mathrm{i}+1}>7 \mathrm{~mm}\right.$.) and no change $\left(-7 \mathrm{~mm} . \leq \mathrm{P}_{\mathrm{i}}-\mathrm{P}_{\mathrm{i}+1} \leq 7 \mathrm{~mm}.\right)$.

The randomization was used to estimate the HO distribution of 144 (mis)matches. The randomization did not produce exactly the same frequencies of underpredictions, overpredictions and matches as the empirical distribution, the randomized distribution resembled the empirically distribution. If it is submitted to a $\chi^{2}$ test, as was done in the Amtz \& van den Hout study (1988), the result is significant $\left(\chi^{2}(4)=17.81, p<0.01\right)$, despite the fact that it was based entirely on chance.

The distribution produced by the randomization procedure was used as a reference to test the empirically observed distribution in a $\chi^{2}$ test (the frequencies produced by the randomization were used as estimates of the expected frequencies under $\mathrm{HO}$ ). The empirical relationship was stronger than the relationship produced by the randomization $\left(\chi^{2}(4)=16.33, p<0.01\right)$.

\footnotetext{
OThis criterion is higher than the $2 \mathrm{~mm}$. criterion proposed by Rachman. It was determined empirically in order to obtain approximately equal frequencies in each cell of the crosstabulation. With the $2 \mathrm{~mm}$. criterion the number of matches was very small. A reason for this deviation from laboratory studies may be the larger interval between ratings of prediction and experience in this study (up to several days).
} 
One could argue that the numbers of underpredictions, overpredictions and matches should be equal in both distributions. Therefore, the randomization was wsed to estimate the $\mathrm{HO}$ distribution assuming the same numbers of underpredictions, overpredictions and matches as in the empirical findings. The resulting distribution is depicted in figure 2 , as is the empirical distribution. As can be seen in the figure, the empirical distribution is still stronger than the HO distribution $\left(x^{2}(4)=12.60, p=0.013\right.$ ): after an underprediction, there were more increases in prediction and fewer decreases in prediction than expected under HO; whereas after overprediction, there were more decreases in prediction and fewer increases in predictions. However, the differences are small. Thus, there is some evidence that the patients were more influenced in forming new predictions by their experiences than can be explained by random processes only.

\section{STUDY 2}

The data in this study are from 46 normal $S$ s who participated in a laboratory study on pain (Amtz \& Lousberg, 1990). Each $S$ received 20 painful electric shocks and rated experienced and predicted pain on $100 \mathrm{~mm}$. VASs. As in study 1 , it was assumed that each $S$ generated predictions randomly from his/her own private distribution. It was decided to use a different statistical procedure to control for the regression effect that can be expected under HO. By means of a stepwise multiple regression analysis we tested if the experience of the $S$ added any significant information to the regression equation, when the previous prediction level was controlled for (that is, controlling for the regression to the mean effect). The variable expressing the regression effect was the individual difference between prediction $\mathrm{P}_{\mathrm{i}}$ and the mean (individual) prediction level $\mathrm{M}$. The change in prediction was expressed as $\mathrm{P}_{\mathrm{i}+1}-\mathrm{P}_{\mathrm{i}}$. Thus, first the regression model,

$$
P_{i+1}-P_{i}=B_{0}+B_{1} \times\left(P_{i}-M\right)
$$

was tested.

Table 1. Regression analysis of changes in prediction controlling for regression to the mean.

\begin{tabular}{lrrrr}
\hline variable & $\beta$ & $d$ & $d . f_{0}$ & $p$ \\
\hline step 1 $\left(\mathbf{R}^{2}=0.21\right)$ & & & & \\
$\mathbf{P}_{\mathrm{i}}-\mathrm{M}$ & -0.36 & -15.72 & 918 & $<10^{-4}$ \\
& & & & \\
$\operatorname{step~} 2\left(\mathbf{R}^{2}=0.57\right)$ & & & & \\
$\mathbf{P}_{\mathrm{i}}-\mathrm{M}$ & -0.14 & -7.49 & 917 & $<10^{-4}$ \\
$\mathbf{P}_{\mathrm{i}}-\mathrm{E}_{\mathrm{i}}$ & 0.54 & 27.40 & 917 & $<10^{-4}$ \\
\hline
\end{tabular}

Note: $\mathbb{P}_{i}-\mathbf{M}$ denotes the difference between prediction of trial $i$ and the mean prediction of the subject. This factor controls for regression to the mean. $P_{i}-E_{i}$ denotes the discrepancy between prediction and experience of trial $\mathrm{i}$. This factor represents the hypothesized mismatch effect. The dependent variable was $P_{i+1}-P_{i}$, the change in next prediction.

It was expected that $\mathbb{B}_{1}$ would be negative (a high $\mathbf{P}_{i}$ would be generally followed by a smaller $P_{i+1}$; a small $P_{i}$ by a larger $\left.P_{i+1}\right)$. And indeed, this was found, as is shown in table 
1. Again, the regression effect seems to explain the match/mismatch model.

However, entering the discrepancy between prediction $P_{i}$ and experience $E_{i}$ into the equation dramatically changed the equation, as is shown in table 1 . Thus, in the regression model

$$
P_{i+1}+P_{1}=B_{0}+B_{1} \times\left(P_{1}-M\right)+B_{2} \times\left(P_{i}-E_{i}\right)
$$

the most important information is yielded by the discrepancy between prediction and experience, precisely as the model states.

\section{DISCUSSION}

Although the $\mathrm{HO}$ model, assuming no 'real' influence of discrepancy between prediction and experience on next prediction, produced effects that are hypothesized by the match/mismatch model, there is clear evidence that the discrepancy is important in the adjustment of predictions as empirically found. That is, Ss generally adjust their predictions if there is such a discrepancy; even if they made an extreme prediction, they did not adjust their predictions if the experience had been correctly predicted. Furthermore, the regression analysis clearly indicates that the size of adjustment of prediction is not so much related to the extremeness of the prediction, as to its inaccuracy. Therefore, there is convincing evidence that the match/mismatch model reflects a 'real' psychological process and not some chance process.

Given the important part that is attributed to predictions in modern learning theory (e.g., Gray, 1975), and in cognitive theories, this is an important finding. It supports the notion that predictions are more than epiphenomena. With respect to fear, anxiety, pain and aversive experiences in general, it is supposed that predictions play an essential part in such areas as dishabituation, anticipatory anxiety, excessive emotional suffering, escape and avoidance behaviour. The match/mismatch model offers insight in the way predictions are changed. Of considerable importance are the conditions that do not lead to the adjustment of inaccurate predictions, as can be observed in clinical problems (Rachman \& Bichard, 1988; Arntz et al., 1990a). It is interesting to note that both the match/mismatch model and the $\mathrm{HO}$ (random) model would predict that very high inaccurate predictions should decrease quickly. In many clinical cases, however, this is not the case, which gives further evidence that the $\mathrm{H} 0$ model cannot be adequate and that there are psychological influences on expectation.

The results of study 1 gave less evidence for the model than those of study 2 . A number of reasons may be offered. In study 1, the patients had to make predictions of 10 different exercises, which may have produced excessive error variance, whereas in study 2 , ss had to make predictions about the same pain stimulus. However, the most important reason is probably that in study 1 patients had to make predictions several days before the exercise, whereas in study 2 the time interval between prediction and experience was less than one mintite. Since predictions probably are state dependent, asking $S$ s to predict how anxious they will be several days before the event is an imprecise procedure. Therefore, stronger support may have been found if the patients had been asked to rate predictions just before the exercise.

Finally, our research indicates that more stringent tests for analyzing the effects of spontaneous mismatches should be used than has been done in the past. It should be kept in mind that under $\mathrm{HO}$ the effects seem to support the model, whereas the model (if it 
does more than describe chance findings) is not implied. More stringent tests can be done in a number of ways. Probably the most simple way is to employ the regression analysis as described above, where the regression to the mean effect is controlled. If an analysis of a cross-tabulation is needed, the probabilities under HO should be estimated (for instance by randomization) and used as a reference to test the empirical distribution. 
$\because$

๖ 


\section{SECTION 2.3. \\ THE EFFECTS OF INCORRECT PAIN EXPECTATIONS ON ACQUIRED FEAR AND PAIN RESPONSES ${ }^{1}$}

\section{SUMMARY}

Previous experimental research on the effects of incorrect intensity expectations of aversive events can be criticized because intensity expectations were not manipulated independently from changes in objective intensity. The present study aims at investigating the effects of incorrect intensity expectations on the immediate and later responses to a painful stimulus, and on the acquisition of anticipatory responses, with proper experimental control. Subjects $(n=62)$ received 20 painful stimuli of varying intensity. In the control group intensities were correctly signalled at all trials by an analogue signal. On 3 trials the signal was too large in the overprediction condition, and too small in the underestimation condition. Underpredicted painful experiences were found to be related to subsequent higher pain responses on the physiological level, but not on the subjective level; and to increased anticipatory responses (increased pain expectations, uncertainty, subjective fear, skin conductance responses). Skin conductance level also indicated increased fear after underpredicted experiences. Overpredicted painful experiences were related to a faster decrease in subjective fear compared to the control group, but did not influence other variables. The findings support the notion that underpredictions contribute to the acquisition of fear and disrupt habituation processes. The asymmetrical processing of the two kinds of incorrectly predicted experiences is discussed.

\section{INTRODUCTION}

For more than three decades the effects of predictability of the occurrence of painful events have been investigated ${ }^{2}$. Recently, the effects of incorrect expectations of the intensity of aversive events have received renewed interest, starting with observations by Rachman and coworkers that underpredicted aversive experiences are followed by increases in predicted intensity of the aversive event and increased anticipatory fear; and that overpredicted experiences are followed by decreased predictions and decreased fear (Rachman \& Bichard, 1988). On the basis of these observations a mismatch model was formu-

'Submitted for publication by Arntz, A., van den Hout, M., van den Berg G. and Meijboom, A.

${ }^{2}$ The general conclusion that can been drawn from this research is that predictability of occurrence reduces fear of and physiological responding to the aversive event, but that it is unlikely that the experienced painfulness of the event is altered by making the occurrence of the painful UCS predictable (for reviews: Furedy, 1975; Miller, 1981; Mineka \& Hendersen, 1985). We traced 43 studies in the literature, 27 of them investigating the effects of predictability of occurrence on physiological pain responses, 28 of them investigating the effects of predictability of occurrence on subjective pain experience. Physiological responses are typically smaller when the stimulus is predictable (observed in 20 studies, whereas 6 studies found no difference and one study the opposite effect). Subjective experience is typically not influenced (as found in 15 studies; 11 studies found a positive effect of predictability, and 2 a negative effect). 
Jated, stating that the changes in predicted aversiveness and in anticipatory fear are a function of the discrepancy between predicted and experienced aversiveness.

It has been pointed out that the amounts of change in prediction and in fear are linearly related to the size of the mismatch (see Rachman \& Bichard, 1988, and Rachman \& Arntz, 1991, for reviews). Closer inspection suggests however that the model can be reduced to a simpler model, which does not need the intervening variable of expectation (which is called 'prediction' when the $S$ makes an explicit rating of it). For if the amount of change in prediction, say $P_{i}-P_{i-1}$, from the subsequent experiences $i-1$ to $i_{n}$ is equal to the discrepancy between experienced and predicted intensity of the event, say $\mathrm{E}_{i-1}-\mathrm{P}_{i-1}$, the model reduces to $P_{i}=E_{i-1}$, in other words: expectations are equal to the last experience (as already observed by Arntz \& van den Hout, 1988, p. 219). Similarly, if the change in subjective fear, $F_{i}-F_{i-1}$, is linearly related the size of the mismatch, $E_{i-1}-P_{i-1}$, the model can be reduced to $F_{i}=c x E_{i-1}+k$, where $c$ and $k$ are constants. In other words, fear of the next experience would be solely based on the previous experience, and there would be no influence of the discrepancy between experience and expectation. If this would be true, the effects of incorrectly predicted experiences would be reduced to the effects of the experience itself - the concept of expectancy would be theoretically empty, and the model would, although correctly, merely describe epiphenomena.

The objection can be made that this simple symmetrical model does not cover the facts. After an underpredicted experience, Ss generally increase their predictions to this lower level. The same asymmetry can be observed in subjective reports of fear. Still, the effects could be ascribed to the stronger pain experience per se, and not to the discrepancy between prediction and experience. It is conceivable that an intensity painful experience, whether correctly predicted or not, influences later pain predictions and fear of pain more strongly than a weaker pain experience. Although a theoretical construct of memory or expectation is needed to explain this asymmetry, it does not necessarily follow that the discrepancy between predicted and experienced pain is a causal factor in this process.

Previous tests of the effects of incorrect predictions on subsequent predictions and fear seem unsuitable to clarify this issue. Some studies investigated spontaneous mismatches. These studies are problematic because statistical artefacts may lead to spurious correlations (Arntz et al., 1990c), Furthermore, without experimental manipulation only correlational interpretations can be made. In other studies, underpredictions were experimentally induced by an unannounced temporary increase in objective stimulus intensity in the experimental conditions (Arntz \& van den Hout, 1988; Arntz \& Lousberg, 1990; Arntz et al., 1991b). Because the control groups did not receive this temporary increase, it is possible that the observed effects of the unannounced increased stimulus (namely, increased pain expectations, a disruption in the reduction of fear and uncertainty, and (some) disruption of habituation to the stimulus) are not caused by the discrepancy between expectation and experience, but by the stronger stimulus itself. A later study (Arntz et al., 1991c) used a better procedure to control for objective intensity (the control group received always strong stimuli, and the experimental condition only on a few (unannounced) trials). But, it is still possible that the observed effects (stronger fear in the experimental group), are caused by the sudden increases in intensity of the stimulus per se, instead of by the underprediction of the experience. It is conceivable that the (autonomic) nervous system is sensitive for sudden increases in intensity of a stimulus, and that such sudden changes cause fear, whereas the discrepancy between the consciously made prediction and the experience, though correlated to a sudden change, is not involved in the acquisition or extinction of fear. The only way to clarify this issue seems to be the experimental manipu- 
lation of incorrect expectations independent from the objective intensity of the aversive stimulus. This is the first aim of the present study.

The second aim of the present study is to investigate the effects of experimentally induced overpredictions (which was not done in previous studies). This may clarify whether the effects of overpredictions are identical to those of underpredictions, or whether there are asymmetries between both kinds of mismatches, as has been suggested by Arntz \& van den Hout (1988).

The third aim of the present study is the investigation of the effects of incorrect predictions on the immediate responses to the painful stimulus; and on the pain responses at later trials. It is still unclear whether incorrect pain-intensity expectations influence pain responses or not. Previous attempts to test this issue suffer from the same problem as the studies investigating the effects of mismatches on subsequent expectations and fear: incorrect predictions and pain experiences were not independently manipulated. It has been hypothesized that underprediction might disrupt habituation processes (Arntz \& Lousberg, 1990) and that overprediction may have a functional value (namely promoting habituation. to pain and avoidance of the possible disruptive effects of an underprediction; Rachman $\&$ Arntz, 1991). If underpredicted aversive experiences are in some way more aversive than overpredicted one's, it is understandable why $S$ s tend to overpredict aversive experiences for a considerable time after an underpredicted experience, and why anxious $S \mathrm{~s}$ tend to overpredict pain (as is clearly shown by anxious dental patients). However, as was pointed out by Rachman \& Armtz (1991), a direct test with a manipulation of pain expectations independent of objective intensity is needed to clarify the influence of pain expectations on pain responses and their habituation.

What should one presume about the effects of incorrect pain expectations on pain impact? There are at least four theories that suggest different answers. First, stimulus representation theories assume that the organism forms a mental model ('stimulus representation") of important aspects of a stimulus. If the incoming stimulus matches this model, it is assumed that the responses elicited by the stimulus are actively inhibited (suppressed). Thus, it has been proposed that the more the stimulus is predictable in important aspects, the better the stimulus can be matched against its model, and the better the inhibition of the responses will be (Gray, 1975; Miller, 1981). It should be noted, however, that this class of theories is mainly based on research into the so-called orienting reflex (elicited by all sorts of new stimuli) and it is less clear whether it also applies to responses typically elicited by painful stimuli, let alone to the experience of pain ${ }^{3}$.

\footnotetext{
3The suppression of pain responses (including habituation, referring to the waning of responses during repeated stimulation) can take place at various levels of the nerwous system, whereas the levels of the nervous system involved in the orienting reflex are more limited. Empirical evidence for pain-response decreasing effects of predictability is far from conclusive. As stated above, predictability of occurrence reduces fear and physiological responding to the aversive everit (notably SCRs), but it is less likely that the experienced painfulness of the event is altered by it. Making the kind of experience more predictable by giving information about the sensations that are triggered by the event is more likely to reduce the subjective impact of the ewent (Miller, 1981). In a metaanalysis of 21 laboratory and field studies, Suls and Wan (1989) concluded that there is clear evidence that sensory preparation is associated with less experienced pain and distress. "Thus, it is clear that (verbal) information about sensations can reduce the experience of pain, demonstrating that expectations formed in 'consciousness' can influence pain experiences. Generalizing the findings of the predictability of occurrence and of the predictability of sensations studies, it can be presumed that pain-intensity expectations at least influence physiological pain responses and possibly the experience of pain.
} 
Second, a contrast theory can be formulated. an aversive experience is in some way contrasted with expectation and impact is related to the contrast. This may be a "cognitive" effect: the experience is evaluated by comparing it to the expectation, or a "neuro-physiological" effect: the response suppression may be related to the expected pain intensity (such an effect is also predicted by Wagner's habituation theory $(1979,1981)$, if it is generalized to the domain of consciously formed expectations). The contrast theory predicts that pain responses are smaller in case of an overprediction, and larger in case of an underprediction, compared to a correct prediction. Such an effect would also be predicted by theories that state that anxiety decreases pain, for instance via naturally produced morphine-like substances, endorphins (Bolles \& Fanselow, 1980; Arntz et al., 1991a), because expectations of intense pain will be related to increased anxiety. Indirect evidence stems from an experiment by willer \& Emst (1986), in which $S$ s manifested a endorphin-mediated analgesia for painful stimuli of low intensity when waiting for a very intense pain stimulus.

Third, cognitive theories have stressed the influence of (strongly believed) expectations on perceptual and evaluative processes. Experiences may be processed in such a way that the discrepancy between expectation and experience is decreased. Moreover, there are also theoretical views that state that anxiety increases pain (Chapman \& Turner, 1986; Amtz et al., 1991a). Thus, if expectations of intense pain lead to increased anxiety, pain impact may be larger when the $S$ overpredicts the pain compared to a correct estimation.

Fourth, the notion that pain expectations don't influence pain responses at all should also be considered. It is conceivable that pain expectations, at least as formed at higher levels of the nervous system, do not play a part in pain-response suppressing or increasing processes.

Besides the above-mentioned study by Amtz \& Lousberg, we found only two studies experimentally assessing effects of incorrect intensity expectations on pain responses. In the first (Epstein \& Clarke, 1970), physiological responses to a noxious stimulus varied directly with anticipation, whereas the subjective rating of the stimulus was related to the contrast between the real and the expected stimulus (overestimated stimuli were rated as less intense than correctly estimated stimuli). The success of the underestimation manipulation was however questionable. In another experiment Epstein (1971) compared habituation over 5 days to aversive shocks and aversive sounds. According to Epstein, most $S$ s have initially highly different expectancies about a shock (most Ss are very concerned) and a sound (most $S$ s are not concerned). In the early period of exposure self-fulfilling influences of expectancy on physiological and subjective responses were found, whereas in later periods a contrast effect became apparent. However, no direct evidence of the alleged under-, and overpredictions was reported. Moreover, the experimental manipulation was done by using two qualitatively different aversive stimuli, thus it cannot be excluded that the observed effects relate to these differences and not to the incorrectness of the expectations. In other words, a new study with more stringent experimental control seems warranted.

To summarize, the aim of the present study is to overcome some of the difficulties of previous investigations into the effects of incorrect pain-intensity expectations by manipulating expectations independently of pain intensities. Specifically, it was investigated (1) whether incorrect pain-intensity expectations influence the immediate responses to the pain stimulus; (2) whether incorrect pain-intensity expectations influence the long-term pain responses [(dis)-habituation]; and (3) whether incorrect pain expectations influence the development of pain-anticipation responses (pain-intensity expectations, certainty of expectation, fear of the painful stimulus and autonomic anticipatory responses). With respect to 
the development of anticipatory responses, it was hypothesized that underpredictions would have negative effects compared with correct predictions, and that overpredictions would have relatively positive effects compared with correct predictions.

In order to accomplish this aim, pain expectations were manipulated by signalling the pain-intensity by means of an analogous signal. The control condition received correct signals. The $S \mathrm{~s}$ in the two experimental conditions (overestimation and underestimation) received incorrect signals (respectively, too large and too small) at three trials interspersed between 17 trials with signals corresponding perfectly to the objective pain-intensity. It was assumed that expectancies influenced by information provided by the signal are equivalent to expectancies that the $S$ forms by own reasoning.

\section{METHOD}

\section{Subjects}

Subjects were 63 female students, who vollunteered to participate. One $S$ (of the overestimation condition) had to be excluded because of a failure of the computer controlling the experiment: this $S$ did not receive the 13th trial. Mean age was 21.5 yr (SD 2.4). Subjects received a small remuneration for participating.

\section{Materials}

Subjects rated expected pain, certainty of this prediction, and fear of next trial, as well as subjective pain intensity and aversiveness experienced during the stimulus on $100 \mathrm{~mm}$ visual analogue scales (VAS). Ratings were measured in $\mathrm{mm}$. A loose-leaf file contained two alternating types of pages, the first with the expected pain, certainty and fear VASs, the second with the experienced pain and aversiveness VASs, for more than 50 trials.

\section{Apparatus}

Painful stimulation was delivered by a Siemens Neuroton 627 via two Beckman Ag$\mathrm{AgCl}$ electrodes (8 mm diameter), filled with HP Redux Creme, at the $S$ 's right ankle. The skin of the ankle was thoroughly rubbed with HP Redux Creme in order to reduce the resistance of the skin and keep it constant (cf. Tursky, 1974). The electrical stimulation consisted of a train of square waved pulses and had a duration of $2 \mathrm{sec}$. The stimulus had instantaneous rising and fall times. The stimulation produces a painful sensation, and is generally not described as an 'electric shock'.

A PC colour monitor was placed approx. $50 \mathrm{~cm}$ before the $S$, in her line of vision. On the monitor a centered horizontal bar could be shown as indication of the intensity of the next pain stimulus. The red-coloured bar had a height of $10 \mathrm{~mm}$ and a width which could be varied from 0 to 100 percent of $247 \mathrm{~mm}$. An Olivetti M250 controlled the experiment.

\section{Physiological recording}

Skin conductance was measured via two Beckman $\mathrm{Ag}-\mathrm{AgCl}$ electrodes ( $8 \mathrm{~mm}$ diameter), placed on the medial phalanges of the second and third finger of the non-dominant hand. The electrodes were filled with an isotonic paste and were connected with a Beckman Skin Conductance Coupler (type 9844, maximum sensitivity 0.05 microSiemens), 
using the method of constant voltage $(0.5$ Volts). The skin was cleaned with distilled water. The response window was 4 sec after stimulus onset for the pain SCR. Anticipatory SCR was defined as the largest fluctuation during anticipation (between onset of the bar signalling the next pain stimulus and pain onset). SCRs were square root transformed (Levey, 1980).

Respiration was recorded from a Beckman Respiration Belt fastened around the $S$ 's chest and connected to a Beckman Voltage/Pulse/Pressure Coupler (type 9853A). There were no SCRs to the pain stimulus attributable to respiratory irregularities: Anticipatory SCRs attributable to respiratory irregularities were excluded.

\section{Procedure}

After introduction to the laboratory, the $S$ was told that he/she could participate in a study on subjective and physiological reactions to pain. No $S$ refused to participate. The $S$ was seated at a table in a dimly lit, sound-attenuated room. Following fastening of the electrodes the stimulation level was determined by increasing the level in steps of $0.2 \mathrm{~mA}$. After reaching pain threshold, VAS pain-intensity ratings were obtained and the level was further raised until it was rated approximately $80 \mathrm{~mm}$ on the VAS. A 5 min resting period was announced and the $S$ was left alone. Following this, the $S$ was instructed by the experimenter to fill in the 3 anticipation VASs after the presentation of a bar on the screen, and the 2 pain-impact VASs after each stimulation. The $S s$ were told that the objective features of the pain stimulus would be different on various dimensions, such as frequency, wave form, intensity, etc. (in fact only intensity varied), from trial to trial and that the object of the study was to investigate subjective and physiological aspects of these dimensions. The $S \mathrm{~s}$ were further told that the coming stimulus intensity would be indicated by the width of the bar on the monitor in front of them, in order to help them prepare for the coming stimulus. It was however stressed that the widths of the bars were based upon the subjective experiences of previous $S$ s and that the $S$ should always make her own judgement of the pain stimulus, and not copy the judgement of the previous $S \mathrm{~s}$. No information was given about the number of stimuli they would receive. After these instructions were given, the experimenter left the room and the series started. Following the series of 20 trials, the $S$ was debriefed, thanked and paid.

A double-blind procedure was followed, in that the experimenter who instructed the $S$ was unaware of the experimental condition, which was determined by a second experimenter who had no contact with the $S$. This second experimenter formed matched trios on the basis of two variables (the objective stimulation levels corresponding to the $S$ 's pain threshold and the $S$ 'S VAS $=80$ rating) and allocated the first $S$ of a matched trio randomly to one of the 3 conditions (control, overestimation and underestimation), the second $S$ randomly to one of the 2 remaining conditions, and the third $S$ to the last remaining condition.

The stimulation-intensity levels that were used were based on the $S$ 's pain threshold level (denoted by ' $t$ ') and the level corresponding to a rating of appr. 80 on the VAS during the working-up procedure (denoted by ' $m$ '). Twenty stimulations were given with objective intensity levels in mA determined by

$$
t+y_{i} \times(m-t),
$$


with $y_{i}$ denoting the relative intensity of trial $i$. The values of $y_{1}$ were for $i=1,2,20$ : $0.5,0.8,0.2,1.0,0.0,0.7,0.3,1.3,0.9,0.6,0.1,0.7,1.2,0.4,0.9,0.1,1.1,0.5,0.2$, 1.0 .

In the control condition, these levels were signalled via bars on the monitor with widths perfectly correllating with $y_{\text {is }}$ namely $30,42,18,50,10,38,22,62,46,34,14,38,58$, $26,46,14,54,30,18$, and 50 percent of the maximum width of the bar. In the overes timation condition, intensity levels higher than the actual level were signaled at trials 8,12 and 17. The widths of these bars were 99,72 and 99 percent, respectively. In the underestimation condition, intensity levels lower than the actual level were signalled at these trials: bars of 18,14 and 18 percent were used.

The interval during which the bar was shown (the bar disappeared with pain stimulus onset) varied from 11 to $29 \mathrm{sec}$ with a mean of $20 \mathrm{sec}$. After pain offset an interval varying from 21 to $39 \mathrm{sec}$ with a mean of $30 \mathrm{sec}$ was employed before the next bar was presented. Intervals were quasi-randomly varied so that each $S$ s, and - within each condition each trial, had a mean interstimulus interval of $20 \mathrm{sec}$ and a mean intertrial interval of 30 sec.

\section{Statistical analysis}

It was planned to use for each variable the mean response to the first seven trials (before the experimental manipulation) as a covariate. The hypotheses were tested by means of MANCOVA's with two planned contrasts, namely the overestimation condition ws the control condition and the underestimation condition is the control condition. The responses of the nonexperimental trials were collapsed in four blocks (block I: trial 1-7; block II: trial 9-11; block III: trial 13-16; block IV: trial 18-20) and analyzed by means of MANOVA trend analyses with the first block as a covariate. Linear and higher order trends will only be reported if the experimental effects on them are not already reflected by the zeroorder trend.

\section{RESULTS}

\section{Pretest differences}

The objective intensity levels of pain threshold and of the VAS $=80$ level differed $n . s_{\text {n }}$ between conditions (mean $=1.05 \mathrm{~mA}, F(2,59)=0.08$; mean $=2.74 \mathrm{~mA}, F(2,59)=0.28$ ), as did the sikin conductance level (SCL) at the end of the resting period $(F(2,59)=0.36)$. The mean responses to the first seven trials (before any experimental manipulation was done) differed $n, s$. between conditions (predicted pain $F(2,59)=0.14$; certainty $F(2,59)=1.10$, $p=0.34$; fear $F(2,59)=0.35$; anticipation SCR $F(2,59)=0.06$; experienced pain-intensity $F(2,59)=0.09$; experienced aversiveness $F(2,59)=0.59$; pain SCR $F(2,59)=1.68$, $p=0.20$ ). Because of the $n . s$. condition effects and the relatively large between $S$ variances, it was decided to use the mean responses to the first seven trials as covariates for the analysis of the later trials.

\section{Manipulation check}

Figure 1 depicts the mean expected pain and fear ratings, as well as the anticipatory SCRs at the experimental trials 8, 12 and 17 (adjusted for the covariates). The experimen- 
tal effects were significant at each of these trials. Therefore, responses were collapsed over these trials. Expected pain was significantly higher in the overestimation condition than in the control condition $(t(38)=7.26, p<0.0001)$, and significantly lower in the underestimation condition compared to the control condition $(t(39)=-18.3, p<0.0001)$. Subjective fear was also higher in the overestimation condition than in the control group $(r(38)=2.86, p<0.01)$. Ss in the underestimation condition rated less fear than the control $S s$ at the experimental trials $(t(39)=-6.98, p=0.0001)$. Anticipatory SCRs were stronger in the overestimation condition than in the control group $(t(38)=2.65, p=0.01)$, and weaker in the underestimation condition than in the control condition $(t(39)=-2.90$, $p<0.01$ ). In sum, subjective expectancy and fear ratings as well as anticipatory SCRs indicate that the experimental manipulation was effective.

\section{ANTICIPATION OF EXPERIMENTAL TRIALS}

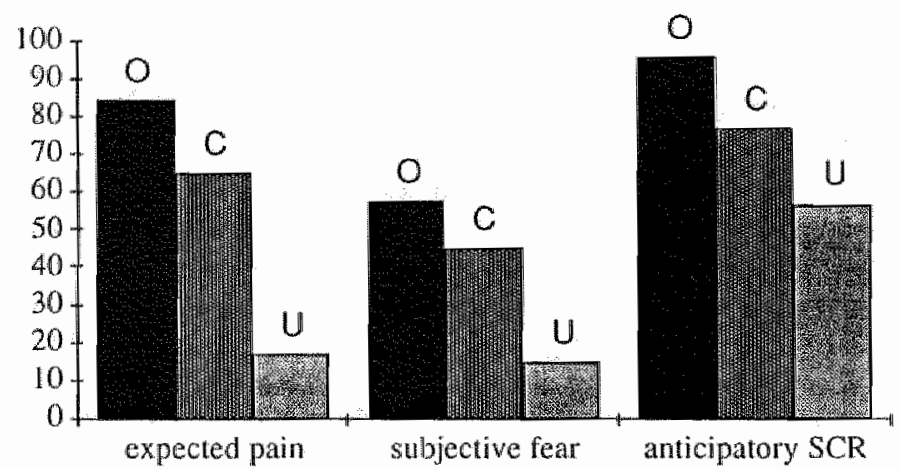

Figure 1. Mean anticipation of the experimental trials (adjusted means). Pain-intensity predictions, subjective fear and anticipatory SCRs were significantly influenced by the experimental induction: they were higher in the overestimation condition $(\mathrm{O})$, and lower in the underestimation condition (U), compared to the control condition, which received correct signals. (SCRs are expressed in microSiemens ${ }^{1 / 2} \times 100$ ).

\section{Immediate influences of incorrect information}

\section{Subjective pain}

The mean pain ratings of the experimental trials are depicted in figure 2 (adjusted for the covariate). Ratings were collapsed over these trials. The planned contrasts revealed that the overestimation condition did not differ from the control condition $(t(38)=0.13)$. The underestimation condition had lower mean pain ratings than the control group $(t(39)=-3.37, p<0.002)$. To summarize, the underestimation information was associated with lower pain ratings compared to the control condition. There was no effect of overestimation information.

\section{Subjective aversiveness}

As is shown in figure 2, the mean aversiveness ratings of trials 8,12 and 17 were less influenced by the experimental manipulation than the pain-intensity ratings. The overestimation condition did not differ from the control condition $(t(38)=-0.15, p=0.61)$ and the underestimation condition did just differ significantly from the control condition $(t(39)=-$ $2.01, p<0.05$ ), showing relatively lower aversiveness ratings. 


\section{Impact SCR}

Figure 2 allso depicts the mean SCRs to the pain stimulation at the experimental trials. Both experimental conditions showed stronger SCRs to the pain stimulus at the experimental trials than the control condition, but the differences were n.s. (overestimation ws control: $t(38)=0.87)$; underestimation vs control $t(39)=0.58$ ). Thus, incorrect pain-intensity expectancies did not result in significantly larger or weaker SCRs compared to correct expectancies.

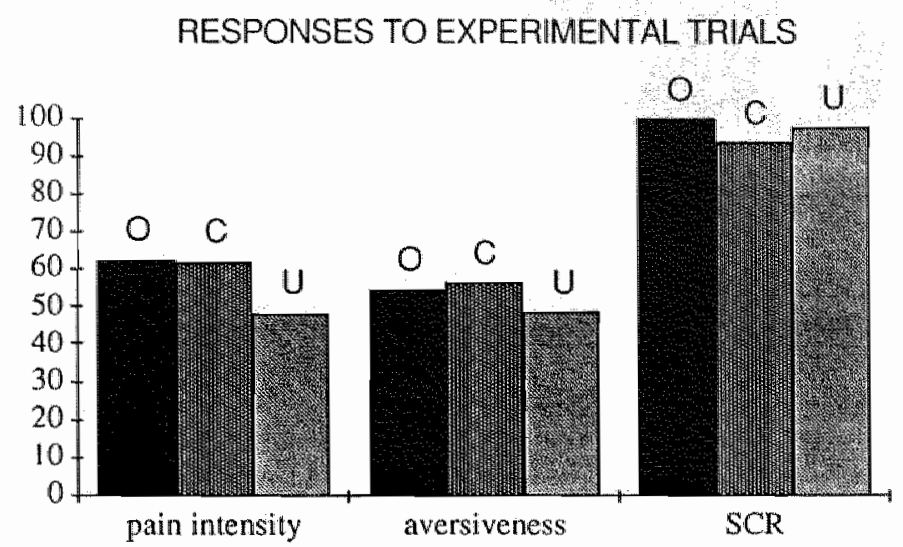

Figure 2. Mean responses to the experimental trials (adjusted means). Subjective ratings were lower when the stimulus was underpredicted (U) than when correctly expected (C) or overpredicted $(\mathrm{O})$. SCRs were not influenced by incorrect expectations. (SCRs are expressed in microSiemens $\left.^{1 / 2} \times 100\right)$.

\section{Indirect influences of the incorrect information}

\section{Subjective pain}

The mean pain ratings of the non-experimental trials 9-11, 13-16, 18-20 are depicted in figure 3 (adjusted for the covariate). There was no experimental effect on the mean pain ratings of these trials: both contrasts were n.s. ( $p$ 's $>0.35$ ).

\section{Subjective aversiveness}

Figure 3 shows that aversiveness ratings of the non-experimental trials were not influenced by incorrect information. There was no experimental effect on the mean aversiveness ratings $(F(2,58)<1$; planned contrasts n.s.).

\section{Impact SCR}

A trend analysis on the SCRs to non-experimental trials collapsed in 4 blocks showed that the underestimation condition differed significantly from the control condition with respect to the linear trend $(t(39)=2.24, p<0.05)$. The underestimation condition showed a slower decrease in SCRs to the pain stimuli than the control group (figure 4). 
RESPONSESTONON-

EXPERIMENTAL TRIALS

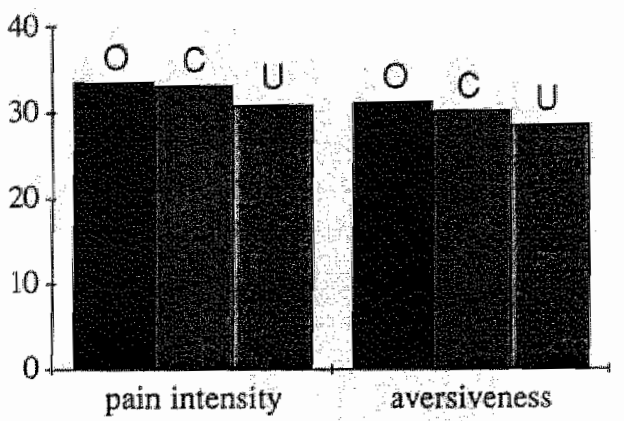

Figure 3. Mean subjective ratings of the nonexperimental trials 9-11, 13-16, \& 18-20 (adjusted means). The overestimation $(O)$ and the underestimation $(U)$ condition did not differ signifeantly from the control group (C).
CHANGES IN SCRS TO PAIN

STIMULUS

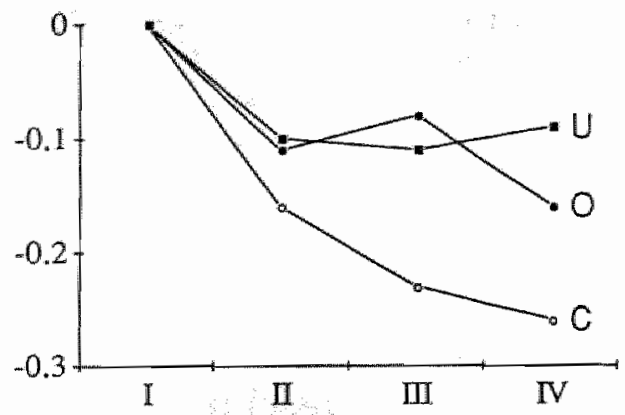

Figure 4. Mean pain SCRs to the nonexperimental trials depicted in 4 blocks (I: trial 17; II: trial 9-11: trial 13-16; IV: trial 18-20). For illustrative reasons, the SCRs are depicted as changes (in microSiemens ${ }^{1 / 4}$ ) with respect to black I. Compared to the control condition (C), the underestimation condition (U) shows less habituation of the SCR to the pain stimulus. The overestimation condition (O) did not differ significantly from the control condition, nor from the underprediction condition.

Although the overestimation condition showed a pattern initially similar to that of the underestimation condition (figure 4), the trends did not differ significantly from those of the control group $(t$ 's $>0.10)$. More specifically, the linear trend did not differ significantly between these conditions $(t(38)=1.56, p=0.12$; note the smaller responses in the overestimation condition during block IV). On the other hand, post-hoc tests revealed that the linear trends in both experimental conditions did not differ significantly $(t(38)<1)$, whereas the contrast between both experimental conditions $v s$ the control condition was significant $(t=2.20, p<0.05)$. Thus, a clear disruption of habituation of SCRs to the pain stimulus was observed in the underestimation condition, whereas the findings in the overestimation condition were equivocal.

\section{Subjective fear of the next stimulus}

Figure 5 depicts subjective fear ratings in four blocks of non-experimental trials. The planned contrasts revealed that the overestimation condition did not differ from the control group as to the average fear rating $(t(38)=-1.33, p=0.19)$, but the underestimation condition had on the average higher fear ratings than the control group $(t(39)=3.73, p<0.001)$. A trend analysis revealed significant experimental effects on the linear trends $(F(2,58)=10.04, p<0.001)$. The overestimation condition differed from the control condition with respect to the linear trend $(t(39)=-2.30, p<0.05)$. The overestimation condition showed a decrease in fear (the linear decrease within this condition was significant, 
$t(18)=-2.67, p<0.01$ ), which was not shown by the control condition $(t(19)=0.55$, $p=0.58$; figure 5). The underestimation condition also differed from the control condition with respect to the linear trend $(a(39)=2.22, p<0.05)$, which was positive within the underestimation condition $(t(19)=3.69, p<0.001)$ and $n . s$. within the control group.

To summarize, overestimation was related to a stronger decrease in fear at the nonexperimental trials than the correct information, whereas the underestimation led to a steady increase in fear.

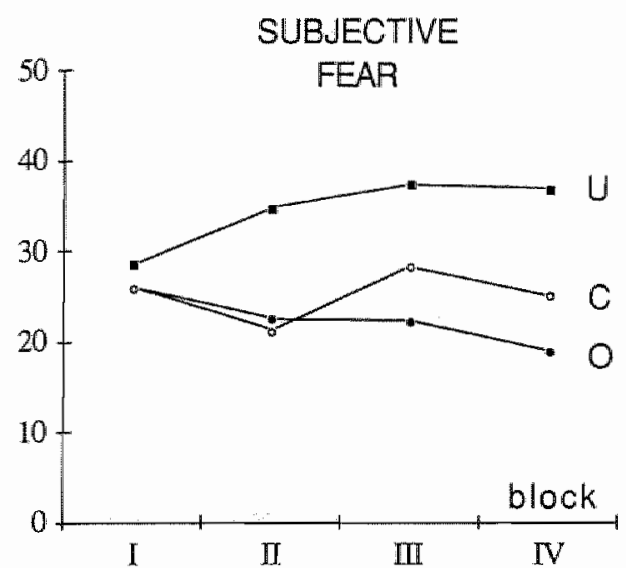

Figure 5. Subjective fear of the next trial in 4 blocks corresponding with the nonexperimental trials $1-7,9-11,13-16, \& 18-20$. The underestimation condition (U) shows an increase in fear, whereas the overestimation condition shows a decrease in fear. Both conditions differed significantly from the control condition (C), which shows a constant level of fear.

\section{PAIN EXPECTATIONS}

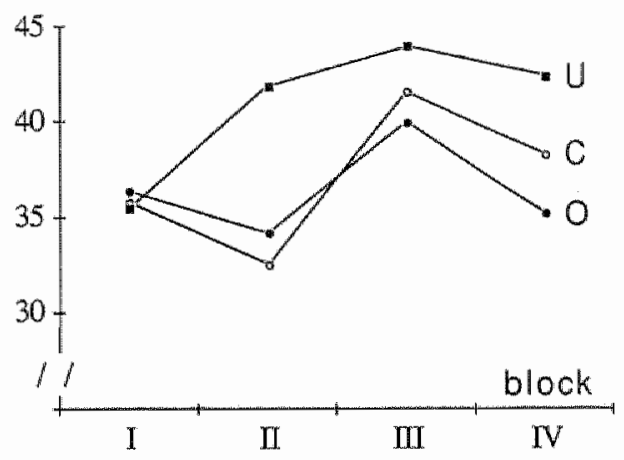

Figure 6. Expected pain in 4 blocks of nonexperimental trials. Pain expectations were higher in the underestimation condition (U) than in the control condition (C). Pain expectations in the overestimation condition (O) did not differ from those of the control group.

\section{Expected pain ratings}

Figure 6 depicts the mean expected pain ratings in 4 blocks of non-experimental trials. The overestimation condition did not differ significantly from the control condition $(t(38)=-0.93, p=0.36)$, but the underestimation condition had higher pain predictions during the last 3 blocks than the control condition $(t(39)=2.93, p<0.005)$. The discrepancy between expected and experienced pain also differed significantly between the underestimation and the control condition during these blocks $(t(39)=3.88, p<0.001)$. The contrast between overestimation and control condition was n.s. $(t(39)=-1.08, p=0.28)$. Thus, there is clear evidence that underestimation led the $S s$ to increase their pain expectations so that they overpredicted their experiences. There was no clear effect of the overestimation manipulation.

\section{Certainty of prediction}

The planned contrasts revealed a n.s. effect of overestimation $(t(38)=-0.11)$ and a significant effect of underestimation $(t(39)=-3.92, p<0.005)$ on the mean ratings of certainty of prediction of the nonexperimental trials. 


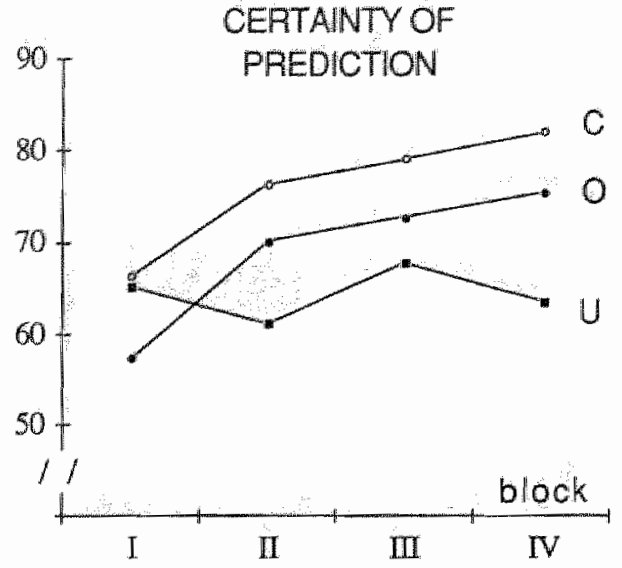

Figure 7. Certainty of pain-intensity prediction in 4 blocks of nonexperimental trials. The underestimation condition (U) remained at a lower level of certainty than the control condition (C), whichtogether with the overestimation condition $(O)$ - increased in certainty.

As is shown in figure 7 , the underestimation condition was more uncertain than the control condition, and did not show an increase in certainty, whereas the overestimation and the control conditions showed linear increases in certainty (linear trend: underestimation vs control contrast: $t(39)=-3.12, p<0.005$; overestimation vs control contrast: $t(38)=$ $0.49, p=0.43$ ). In sum, the hypothesis that underestimation leads to uncertainty about next pain predictions is supported.

\section{Anticipatory SCRs}

A trend analysis revealed no significant differences between overestimation and control conditions $(p ' s>0.40)$. The underestimation condition differed, however, from the controi condition in the mean $(t(39)=2.04, p<0.05)$, quadratic $(t(39)=-1.84, p=0.07)$ and the cubic trend $(r(39)=2.08, p<0.05)$. As is shown in figure 8 , anticipatory SCRs decreased more slowly in the underestimation condition than in the control condition.

$S C L$

As is shown in figure 9, the mean SCL during the intertrial intervals between trials 820 increased compared to trials $1-8$ in the underestimation condition (within effect $t(20)=2.06, p=0.05)$, whereas it did not change significantly in both other conditions $\left(t^{\prime} s<1, p\right.$ 's $\left.>0.50\right)$. The contrast between underestimation and control conditions was significant $(r(39)=2.10, p<0.05)$, whereas the overestimation condition did not differ significantly from the control group $(t(38)=0.39)$. In sum, the underprediction manipulation resulted in increased $\mathrm{SCL}$, in contrast to the overprediction manipulation and correct information. 


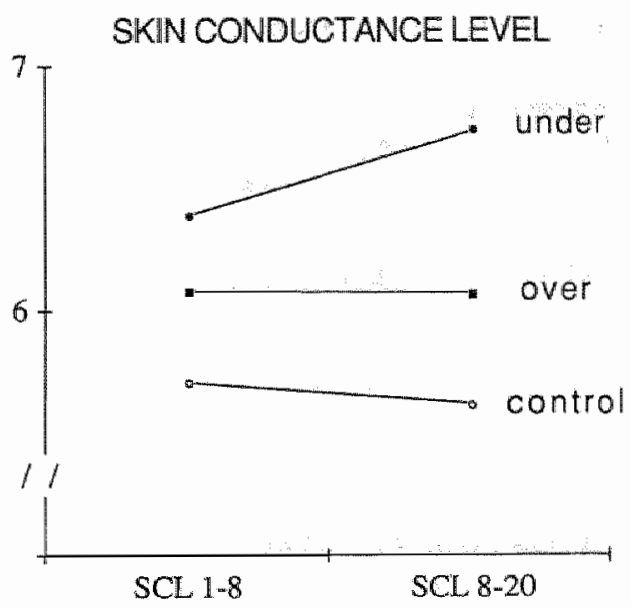

Figure 9. Mean intertrial SCL before (SCL 1-8) and after (SCL 8-20) the first experimentall trial. The underestimation condition showed an increase in SCL due to the experimental manipulation, the other two conditions remained constant.

\section{DISCUSSION}

The present study investigated the effects of incorrect pain-intensity expectations on the impact of the painful stimulus and on the development of anticipatory responses. The results can be readily summarized.

First, with regard to the immediate impact of an incorrectly expected painful stimulus, it was found that only the underestimation information influenced the subjective reports about the incorrectly expected pain stimulus. The overestimation information did not seem to have any influence. In addition, the physiological reaction to this stimulus was not influenced by incorrect information. Second, with regard to the responses to pain stimuli following the incorrectly signaled trials, a discordance between subjective and physiological reactions was found. Subjective reports about the pain stimulus were not influenced, but physiological reactions decreased more slowly in the underestimation condition than in the control condition, whereas the overestimation condition showed a pattern somewhere in between those two. Thus, habituation was interrupted by underestimated experiences, but only at the physiological level. Third, anticipatory responses were clearly influenced by incorrect pain expectations. Overall, the underestimation condition manifested stronger anticipatory responses than the control condition. Pain expectations, uncertainty, subjective fear and anticipatory SCRs were larger in this condition. The overestimation manipulation did not lead to negative effects compared to correct information. Positive effects of overprediction were only observed with respect to subjective fear, which decreased in the overestimation condition, whereas it remained constant in the control condition. That underestimation led to increased fear is also indicated by the increased SCL in this condition, whereas it remained constant in the other two conditions.

Returning to the issue of the influences of incorrect intensity expectations on the immediate impact of a painful stimulus, the results partly support the (cognitive) theory that discrepancies between expectations and experiences are decreased by distorting the experience into the direction of the expectation. This finding should be interpreted cautious- 
ly, however. First, a similar effect was not observed in the overestimation condition. And second, this effect was not found with the SCRs. Thus, the distortion of subjective pain ratings may have been a superficial effect, possibly caused by some sort of social conformity, which may have played a role despite the instruction emphasizing independent judgements. But why did the overestimation condition not manifest such an effect? It is conceivable that this was an effect of the specific series of intensities used in the present study. Before the first experimental trial, the 8th trial, weak intensities were presented, but high intensities were not given. Thus, the underestimation condition had experienced some references for weak intensity signals, whereas the control and overestimation conditions did not have any experience with intensities of the level that was signalled at the 8th trial. The absence of clear anchors for the high intensities signals at the experimental trials in the overestimation condition may have caused an undistorted subjective report of the experience. This possibility gives rise to the question whether expectations that are formed without such clear external anchors have the same influence on immediate pain responses. In fact, in the Epstein (1971) and Epstein \& Clarke (1970) studies, in which under- and overexpectations were induced without a clear external reference, results were found that divert from the present study. In the absence of a signal that serves as a strong external anchor there may be less neccessity to decrease the discrepancy between anchor and experience by distorting the experience, and a contrast effect may become apparent (as was observed in both Epstein studies). Clearly, further research is needed to clarify this issue.

Despite the lower subjective ratings of the underestimated trials, compared to the correctly and over-predicted trials, anticipatory fear and physiological responses to later pain stimuli were larger in the underestimation group than in the other two groups. Thus, the hypothesis that incorrect pain expectations, and especially underpredictions, disrupt habituation processes was supported only on the physiological level. Even for the (chronic) experience of pain this finding may have significance. Various physiological feedback systems, some involving the sympathetic nervous system, can play a role in amplifying pain experiences (Chapman \& Bonica, 1983). Thus, underpredicted painful experiences may in the long run lead to increased pain problems via increased autonomic activity which may influence physiological feedback mechanisms. However, the lack of direct influence of incorrect pain expectations on later pain experiences contradicts earlier suggestions of such an influence (Arntz \& Lousberg, 1990; Rachman \& Amtz, 1991).

The effects of underpredictions match in a considerable extent to the effects of unpredictability of occurrence of painful stimuli. Physiological responses to the pain stimulus, tonic physiological arousal (SCL) and various subjective fear indices were increased in the underestimation condition. The reason seems clear: the $S$ cannot trust the signals anymore, because they are sometimes followed by higher intensities than expected. Clearly, the processing of experiences that are oversignalled differs from those that are undersignaled. Overestimation does not have dramatic effects - more specifically, there is little effect on the subsequent pain predictions and on certainty of predictions. Can modem learning theory explain these findings? Rachman \& Bichard (1988) commented earlier on the similarities between the Rescorla-Wagner conditioning theory and the findings with respect to incorrect expectations of aversive events. In both models, the change in the strength of anticipatory variables is related to the difference between (in some formal way) expected and experienced event. However, Rachman's earlier formulation, as well as the RescorlaWagner (1972) model (and Wagner's later model, see Wagner, 1981, pp 39-40) predict symmetrical effects: discrepancies of both kinds lead to changes in opposite direction but of comparable size. The processing of aversive events follows probably different (asymmetrical) rules, as was already noted by others (Furedy et al., 1983; Rachman, 1991). 
Moreower, from conditioning theory one might speculate that in the underestimation condition fear responses are especially elicited by the small signals (because they were followed by high intensities at the experimental trials). However, a careful (post-hoc) analysis of the fear responses showed that responses to the signals immediately following the experimental trials were most elevated, and not the responses to signals of low intensities. The conclusion seems therefore warranted that the asymmetrical effects of incorrect expectations are not based on 'simple' conditioning to low intensities signals, but on a general distrust of the signal, which gradually reduces with disconfirmatory experiences.

All in all, the present study seems to give further support for various earlier claims. Because pain-predictions and objective stimulus intensities were independently manipulated in the present study, problems with the interpretion of earlier studies do not play a part now. It seems clear now that the discrepancy between expected and experienced pain plays a role in the development of anticipatory responses (and in (habituation of) physiom logical responses to the pain stimulus), and that this effect cannot be reduced to the effects of the experience itself. Moreover, underpredictions and overpredictions seem to have quite different effects: underpredictions are clearly related to increased fear and autonomic responding, but overpredictions neither have a similar effect, nor an opposite effect. The only specific effect of the overprediction manipulation seems to be the faster decrease of subjective fear. The absence of other effects may have various reasons. As was suggested earlier (Arntz \& van den Hout, 1988), the asymmetries between both kinds of incorrectly expected experiences suggest that after an overpredicted aversive experience, $S$ s keep at the safe side and are reluctant to lower their predictions as much as $S \mathrm{~s}$ increase their predictions after an underpredicted experience ${ }^{4}$. Such a reluctance would suggest that it is in some way more aversive to experience an underestimated aversive event than an overestimated/correctly estimated event of equal objective intensity. However, the present study indicates that the immediate and later experience is not negatively influenced by incorrect expectations (although in the long run autonomic responding is influenced). This 'functionality' explanation seems therefore unlikely.

Another possibility is that in some way underpredicted experiences receive a greater weight in the processing of the experience, or are more accessible from memory for formation of new expectations, than correctly and over-predicted events (cf. Rachman \& Amtz, 1991). But what may be the reason of this bias? If it is not related to decreased impact of correctly or overpredicted events, the reason for the asymmetrical processing is probably related to avoidance behaviour. Under conditions of lessened predictability caused by experiences that exceed expectation, it seems safe to avoid future encounters, since the direction of the discrepancy is towards the dangerous side and the stimulus cannot be trusted anymore. If, however, the experience is less aversive than expected, the possible consequences of this loss of predictability are not at all dangerous for the organism. The asymmetrical effects of the two kinds of incorrect prediction on certainty of prediction are in support with this view. It is therefore suggested that the asymmetrical processing of incorrectly expected experiences, manifested as a conservative bias, serves as the basis of conservative overt behaviour (avoidance, preparation for actions like fight/flight). It is interesting to note that in the present study this conservative bias was observed both on the physiological and the cognitive level. Cognitive variables related to

${ }^{4}$ Detailed inspection of the discrepancies between predicted and experienced pain showed that the $S s$ in the overestimation condition did not underpredict any of the trials after the experimental trials, not even the trials immediately after the experimentally induced overprediction. 
expected aversive events do not allways behave so 'rationally' as some authors suggest (e.g. Furedy et al., 1983), which has of course been observed before (e.g. de Jong, Merckelbach \& Arntz, 1991; Mineka \& Tomarken, 1989; Nisbett \& Ross, 1980). 


\title{
SECTION 2.4. \\ PREDICTIONS OF DENTAL PAIN: THE FEAR OF ANY \\ ANY EXPECTED EVIL, IS WORSE THAN THE EVIL ITSELF.*1
}

\author{
* Thomas Nashe (1594) Terrors of the Night.
}

\section{SUMMARY}

In a study of $40 \mathrm{Ss}$, who twice underwent extensive dental treatment, the relationships between expectations and experiences of pain and of anxiety were investigated. Inaccurate expectations were adjusted in the same way as observed in the laboratory. Especially anxious $S$ s expected more pain and anxiety than they experienced, and they appeared to need more experiences before their predictions became accurate. In the course of time, the expectations (and memories) of anxious $S$ s returned to their original more inaccurate level of prediction. The results suggest that the old schema is ultimately reinstated if disconfirmations are few and far between. Anxious Ss did not experience more pain, but they did experience more anxiety than fearless $S \mathrm{~s}$. Detailed investigation of processes of change after disconfirmation showed that anxiety experienced during treatment is a factor that plays a part in maintaining the problem of inaccurate expectations and fear of treatment. Theoretical and clinical implications of these findings are discussed.

\section{INTRODUCTION}

Painful experiences can be unpredictable, with respect to, for instance, moment of occurrence, intensity, duration and consequences. Recent laboratory research concentrating on the consequences of inaccurately predicted intensity of painful experiences may increase our insight into human processing of naturally occurring painful experiences. In short, these studies have shown that inaccurately predicted pain tends to be followed by immediate changes in pain predictions of the next experience. The direction of the change is in accordance with the direction of the mismatch: underpredicted pain is followed by an increase in predicted pain; overpredicted pain is followed by a decrease in predicted pain; and accurate predictions do not lead to changes in predictions (Arntz \& van den Hout, 1988; Rachman \& Lopatka, 1988; Arntz \& Lousberg, 1990). In addition to this, there appear to be dissimilarities between the two kinds of inaccurate predictions: underpredictions generally have stronger effects on subsequent predictions and cause a lasting increase in fear of the painful stimulus (Arntz \& van den Hout, 1988; Arntz \& Lousberg, 1990). A strong underprediction can even have dishabituating effects, sensitizing the $S$ for the following experience (Arntz \& Lousberg, 1990). Overpredictions (which may result from an underpredicted experience) can be relatively protracted, needing more disconfirmations before they become accurate than underpredictions (Rachman \& Bichard, 1988; Arntz \& van den Hout, 1988; Amtz \& Lousberg, 1990). These findings are potentially of clinical relevance: especially clinical pain problems are often characterized by a chronic tendency

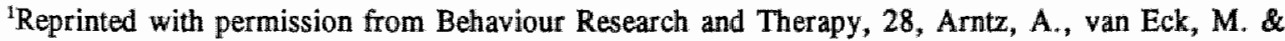
Heijmans, M., Predictions of dental pain: the fear of any expected evil, is worse than the evil itself. 1990, Pergamon Press PLC.
} 
to overpredict pain, which is sometimes accompanied by avoidance behaviour (Kleinknecht \& Bernistein, 1978; Lethem et al., 1983; Slade et al., 1983; Liddell \& May, 1984; Philips, 1987).

Whereas most laboratory findings on the effects of inaccurate predictions are robust, it still has to be shown that these findings also apply to clinical problems. With respect to chronic arthritic pain, Rachman \& Lopatka (1988) showed that underpredictions of pain due to physiotherapeutic exercises were followed by increased pain predictions, overpredictions of pain by decreased predictions, and accurate predictions were not followed by any change in predictions. Most of the inaccurate predictions were overpredictions, as is hypothesized by Rachman's match/mismatch model. The relationship between inaccurate pain predictions and fear of pain was not assessed, however.

Dental treatment is another field in which research into prediction of pain has been carried out. The dental situation offers an excellent opportunity to study the interplay between anxiety and paün. Many $S \mathrm{~s}$ suffer from dental anxiety, sometimes so severely that they avoid dental treatment and suffer from a bad condition of their teeth (Lindsay, 1983). Whereas fearless dental patients appear to have quite accurate expectations as to pain due to dental treatment, fearful dental patients, including dental phobics (who avoid dental treatment), generally overpredict pain of treatment (Kleinknecht \& Bernstein, 1978; Lindsay, 1983; Wardle, 1983, 1984; Kent, 1984, 1985; Liddell \& May, 1984). The most important issue that these patients fear appears to be extreme pain caused by treatment (Lindsay, 1983).

The origins of dental fear are not entirely clear yet. Retrospective research generally indicates onset during childhood or adolescence (Marks, 1987). Liddell and May (1984) found an age of onset with a median of $11.3 \mathrm{yr}$ and a mode of $7 \mathrm{yr}$. Though fearful subjects especially fear pain due to treatment, it is not clear whether they actually had more painful experiences. Prospective research is lacking, and retrospective research shows inconsistent results (Lindsay, 1983). In the literature on the subject, the following factors are often reported: neuroticism as a personality trait; modelling by parents or peers; information from parents or peers; unpleasant or even frightening behaviour on the part of the dentist; unpleasant or painful treatment in childhood (after the first treatment) (Lautsch, 1971; Klepac et al., 1982, Lindsay, 1983; Liddell \& May, 1984). Therefore, it may be possible that an unexpected extremely painful treatment together with other variables (such as frightening behaviour of the dentist) is related to the genesis of dental anxiety and dental phobia. Be that as it may, fearful patients appear to have the general idea that dental treatment is very painful and unpleasant. It seems to be quite difficult to adjust this conception, even though there are disconfirming experiences: in a study by Kent (1985) it was found that 3 months after treatment, the treatment was more painful in fearful patients' memories than was actually experienced and the memories were related more to the original expectation than to the experience. Despite the relative constancy of dental anxiety, decreases are found, and one study found that they were related to disconfirmed high pain expectations (Kent \& Warren, 1985). Decreases in fear and in pain predictions were relatively small, however. Therefore, long-term memories of these experiences seem to be determined rather by the $S$ 's original prediction (or, more generally speaking, by the $S$ "s schema) than by the experience itself. Thus, under certain conditions aversive experiences seem to be processed in a way that leads to chronic overestimation of then. It is still unclear why disconfirming experiences are processed so badly that they fail to influence this schema.

Whereas it is clear that dental fear is related to rather high and inaccurate pain predictions, it is unclear whether high anxiety is related to a more intense pain experience. 
Although this relationship is often assumed to exist, a recent short review of empirical studies provided little evidence to sustain this hypothesis (Arntz \& Schmidt, 1989). In the case of dental pain, a positive correlation between dental anxiety and pain is reported by Kleinknecht \& Bernstein (1978), but most studies show n.s. or even negative relationships (Kent, 1984, 1985; Wardle, 1984; Kent \& Warren, 1985; Chaves \& Brown, 1987). Mixed results, which appear to be inconsistent when the studies are set side by side, are reported by Lautsch (1971), and Klepac et al. (1980, 1982). Therefore, positive findings about a relationship between anxiety and pain are scarce and inconsistent. There is more evidence that there is no direct relationship between subjective anxiety and experienced pain.

The aim of the present study is to make a further investigation into the relationship between expectations and experiences of pain and anxiety, and their relationship to fear of pain, in a clinical setting: the dental situation. Little is known about the process of changes, both immediate and long-term, in predictions and fear of dental pain. Therefore, the present study is limited to the dental procedures that require two successive treatment sessions, both possibly painful. Immediate and follow-up measurements were planned. This procedure will offer the opportunity to study the processing of two successive pain experiences and their immediate as well as their long-term effects in detail.

The hypotheses tested are formulated below. They were derived from previous laboratory studies on the match/mismatch model of fear and pain (cf. Rachman \& Bichard, 1988; Amtz \& van den Hout, 1988; Rachman \& Lopatka, 1988; Arntz \& Lousberg, 1990); from the existing studies on expectations of dental pain; and from what is generally assumed about the interrelationship between anxiety and pain.

\section{Hypotheses}

\section{Matches and mismatches}

1a. Underpredicted pain will be followed by increased pain predictions.

b. Overpredicted pain will be followed by decreased pain predictions.

c. Correctly predicted pain will not be followed by any change in pain predictions.

2a. Underpredicted anxiety will be followed by increased anxiety predictions.

b. Overpredicted anxiety will be followed by decreased anxiety predictions.

c. Correctly predicted anxiety will not be followed by any change in anxiety predictions.

3a. Underpredicted pain will be followed by increased dental anxiety.

b. Overpredicted pain will be followed by decreased dental anxiety.

c. Correctly predicted pain will not be followed by any change in dental anxiety.

\section{The influence of dental anxiety}

4. Dental anxiety is associated with relatively high pain predictions.

5. Subjects with high dental anxiety overestimate pain; fearless subjects predict pain accurately.

6. Subjects with high dental anxiety need several disconfirmations before they predict pain accurately.

7. Dental anxiety is associated with higher experienced pain.

Memory and schemas of pain.

8. Memory of experienced pain and predicted pain several months after treatment will be related more to the original predictions of pain than to the pain that is actually experienced. 
9. Subjects with high dental anxiety have more painful memories of the most painful treatment they ever experienced than fearless subjects.

\section{METHOD}

\section{Subjects and treatments}

The $S$ s were 40 volunteers, 20 men and 20 women, who were all treated twice in succession. The average age was $33.9 \mathrm{yr}$ (range 17-71). The time between the treatment sessions varied from 1 to 37 days (mean 10.4). Both treatment sessions could be painful and the treatment consisted of placement of crowns, root canal work, placement of fillings, etc. Check-ups were excluded. The study was held in the practices of three dentists in Maastricht.

\section{Materials}

At each treatment, the $S s$ were asked to complete two questionnaires, one before and one after treatment. The first one contained a checklist of 13 items about the reasons of dental anxiety and the Dental Anxiety Scale (DAS, Corah et al., 1978). The Ss indicated predicted pain on a visual analogue scale (VAS), a $100 \mathrm{~mm}$ horizontal line ranging from 0 ('not at all painful') to 100 ('the worst pain imaginable'). Predictions of aversiveness of treatment and anxiety during treatment were measured in the same way. Patients also indicated on a VAS to what degree they feared the coming treatment ('0, not afraid at all' to '100, the most intense fear imaginable'). In the second questionnaire, administered immediately after treatment, $S \mathrm{~s}$ indicated pain, anxiety and aversiveness experienced during treatment on VASs, as well as expectations and fear concerning the next treatment. After the second treatment, expectations and fear concerned a hypothesized (similar) third treatment. The follow-up questionnaire consisted of the DAS, VASs on remembered pain, anxiety and aversiveness as to the second treatment, predictions for a following (hypothetical) similar treatment, as well as a VAS measuring remembered pain of the most painful treatment ever experienced.

\section{Procedure}

Patients were asked by the dentists assistant to participate in a study on "the pain people feel when they visit the dentist'. After they had completed the scales in the waiting room, they returned the questionnaire to the assistant in a sealed envelope. Both assistant and dentist were unaware of the scores of the patient. Immediately after the treatment, the $\mathrm{Ss}$ filled in the post-treatment scales in the waiting room and gave them to the assistant, again in a sealed envelope. Five months after the second treatment, the $S$ s received a letter asking them to fill in the enclosed scales and to send them to the university. Since some patients preferred to participate anonymously, not all patients could be contacted. Others still did not respond after a telephone call reminding them of the letter. The follow-up measurements were obtained from 31 of the 40 patients. 


\section{RESULTS}

\section{Matches and mismatches of pain predictions (hypothesis 1)}

The differences (D) between experienced and predicted pain were calculated and classified as overprediction $(\mathrm{D}<-2 \mathrm{~mm})$, underprediction $(\mathrm{D}>2 \mathrm{~mm})$ or match $(-2 \mathrm{~mm} \leq \mathrm{D} \leq 2$ $\mathrm{mm}$ ), on the basis of the criteria by Rachman et al. By means of the same criteria, changes in predictions were classified as increase, decrease or no change.

At treatment 1,27 of the $40 \mathrm{Ss}$ made an overprediction $(67.5 \%), 7 \mathrm{Ss}$ made a correct prediction $(17.5 \%)$ and $6 \mathrm{Ss}$ made an underprediction $(15 \%)$. At the second treatment there were 23 overpredictions $(57.5 \%), 10$ matches $(25 \%)$ and 7 underpredictions $(17.5 \%)$. Thus, there were more overpredictions than underpredictions of pain (1-tailed binomial probabilities under H0: $\pi=0.5$ are $<0.003$ for both sessions). As is illustrated in figure 1 , overpredictions are generally followed by decreases in predicted pain (treatment 1: $66.7 \%$; treatment $2: 78.3 \%$ ), and correct predictions are mostly not followed by any change in predictions $(85.7 \%$ and $80 \%)$. After the first treatment, most underpredictions were followed by an increase in predicted pain $(83.3 \%)$ but, contrary to what was hypothesized, only $28.6 \%$ of the underpredictions was followed by an increased prediction after the second treatment. Most of these underpredictions were not followed by any change in predicted pain $(57.1 \%)$ ). A $\chi^{2}$ test performed on the $3 \times 3$ cross-tabulations yielded highly significant results:" $\chi^{2}(4)=31.72, p<10^{-4}$ (treatment 1) and $\chi^{2}(4)=21.53$, $p=0.002$ (treatment 2).

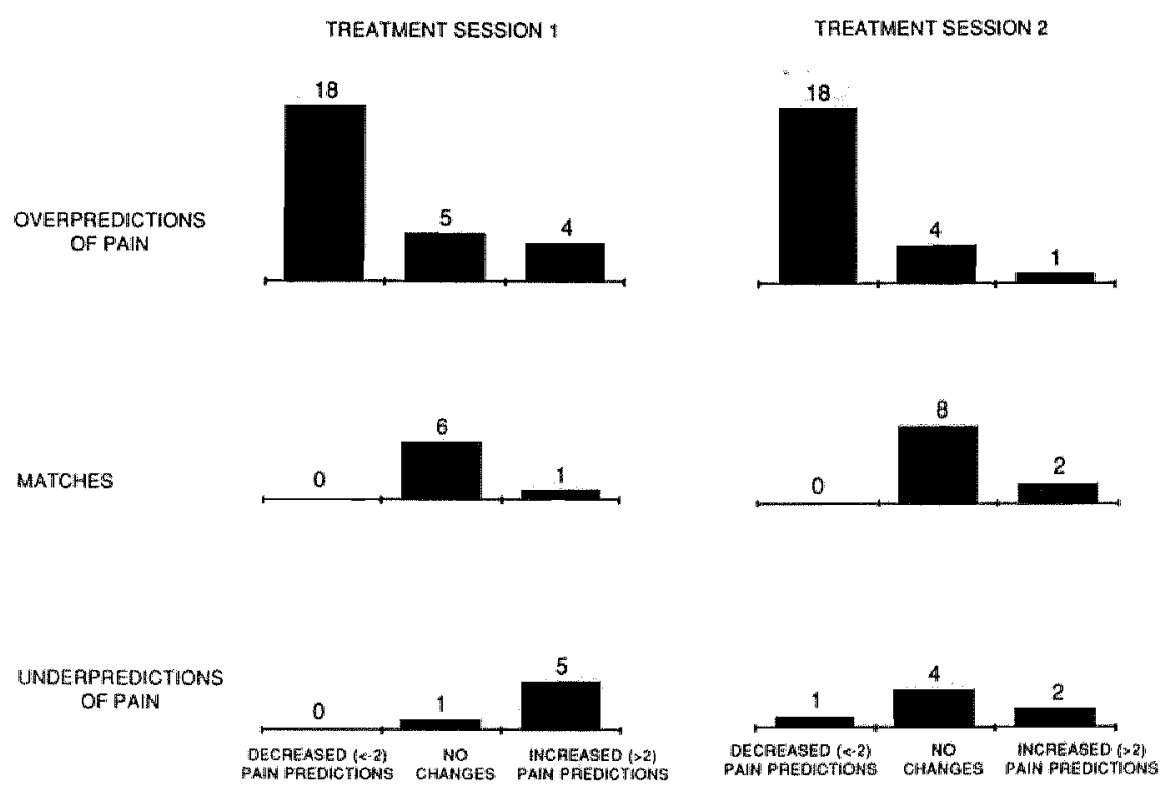

Figure 1. The relationship between inaccurate pain predictions and changes in pain predictions after treatment $1\left(p<10^{-4}\right)$ and treatment $2(p=0.002)$. Most $S \mathrm{~s}$ who made an overprediction decreased their prediction, most $S \mathrm{~s}$ who made a correct prediction did not change their prediction, and after treatment 1 most $S$ s who made an underprediction increased their prediction. 
Regression analyses in which the change in predicted pain was the dependent variable and the discrepancy between predicted pain and experienced pain was the predictor, also showed highly significant relationships: $r=0.54, B=0.56, F(38)=15.85, p=0.0003$ (treatment 1) and $r=0.75, B=0.71, F(38)=46.46, p<10^{-4}$ (treatment 2). The interval between the treatment sessions did not influence the changes in predictions after the first treatment very much: identical tesults were found when predictions just before treatment 2 were used instead of predictions immediately after treatment $1\left(x^{2}(4)=23.18, p=0.001\right.$; $r=0.45, B=0.43, F(38)=9.84, p=0.003)$. Thus, hypotheses la-c are supported for the most part.

\section{Matches and mismatches of predicted anxiety (hypothesis 2)}

Patients were somewhat more accurate in predicting subjective anxiety during treatment than in predicting pain. At the first treatment $24 \mathrm{Ss}$ made an overprediction $(60 \%), 10$ made a match (25\%) and 6 made an underprediction (15\%). At treatment 2 there were less overpredictions $(14,35 \%)$, and more matches $(20,50 \%)$. The number of underpredictions of anxiety remained constant $(6,15 \%)$. The decrease in the number of overpredictions of anxiety is significant $\left(x^{2}(1)=4.06, p=0.04\right)$.

The relationship between matches/mismatches and changes in predicted anxiety is illustrated in figure 2. Overpredictions of anxiety tend to be followed by decreases in predicted anxiety.

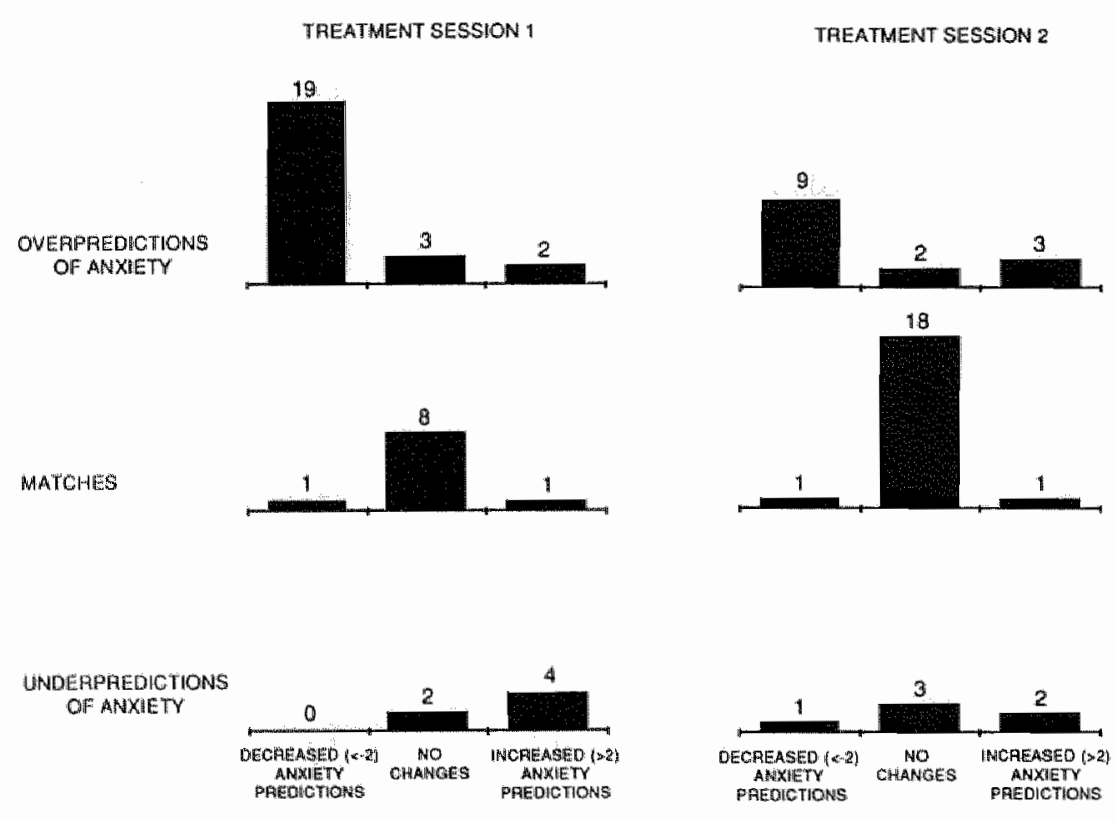

Figure 2. The relationship between inaccurate anxiety predictions and changes in anxiety predictions after treatment $1\left(p<10^{-4}\right)$ and treatment $2\left(p<10^{-3}\right)$. Overpredictions are mainly followed by decreases, correct predictions by no change, and underpredictions by increases (except at treatment 2) in predicted anxiety. 
Following a correct match there are hardly any changes in predicted anxiety. After underpredictions, the findings are similar to those of predictions of pain: after treatment 1 , most underpredictions were followed by increases in predicted anxiety $(67 \%)$, but after treatment 2 most underpredictions were not followed by any change in predictions of anxiety (50\%) and only a minority were followed by increases in anxiety predictions $(33 \%)$. At both sessions, the relationship between matches/mismatches and changes in predicted anxiety is significant $\left(\chi^{2}(4)=29.94, p=0.0001 ; \chi^{2}(4)=22.17, p=0.0002\right)$. Regression analyses show that the degree of change in predicted anxiety is linearly related to the discrepancy between predicted and experienced anxiety: $R=0.76, B=0.91, F(38)=52.18$, $p<10^{-4}$ (treatment 1); $R=0.57, B=0.58, F(38)=17.10, p=0.0002$ (treatment 2). The effect of mismatches at the first treatment was still found in the predictions just before the second treatment $\left(\chi^{2}(4)=17.87, p=0.0013 ; R=0.68, B=0.76, F(38)=33.22, p<10^{-4}\right)$. Thus, hypotheses $2 \mathrm{a}-\mathrm{c}$ are also supported.

\section{The influence of inaccurate pain predictions on dental anxiety (hypothesis 3)}

Overpredictions of pain are followed by decreases in predictions of anxiety, matches are not followed by any change in predictions of anxiety, and underpredicted pain is followed by increases in predictions of anxiety $\left(\chi^{2}(4)=14.62, p=0.006 ; \chi^{2}(4)=12.27, p=0.016\right)$ at both treatment sessions, which can be seen in figure 3 . At the second treatment there were, however, more cases of unchanged anxiety predictions after overpredicted pain and especially after underpredicted pain. It can also be seen in figure 3, that inaccurate predictions of pain at treatment 1 had less effect on general dental anxiety as measured by the DAS. There was not a significant relationship between matches/mismatches of pain predictions at treatment 1 and change in DAS score from treatment 1 to treatment $2\left(\chi^{2}(4)=\right.$ $2.61, p=0.62$ ).

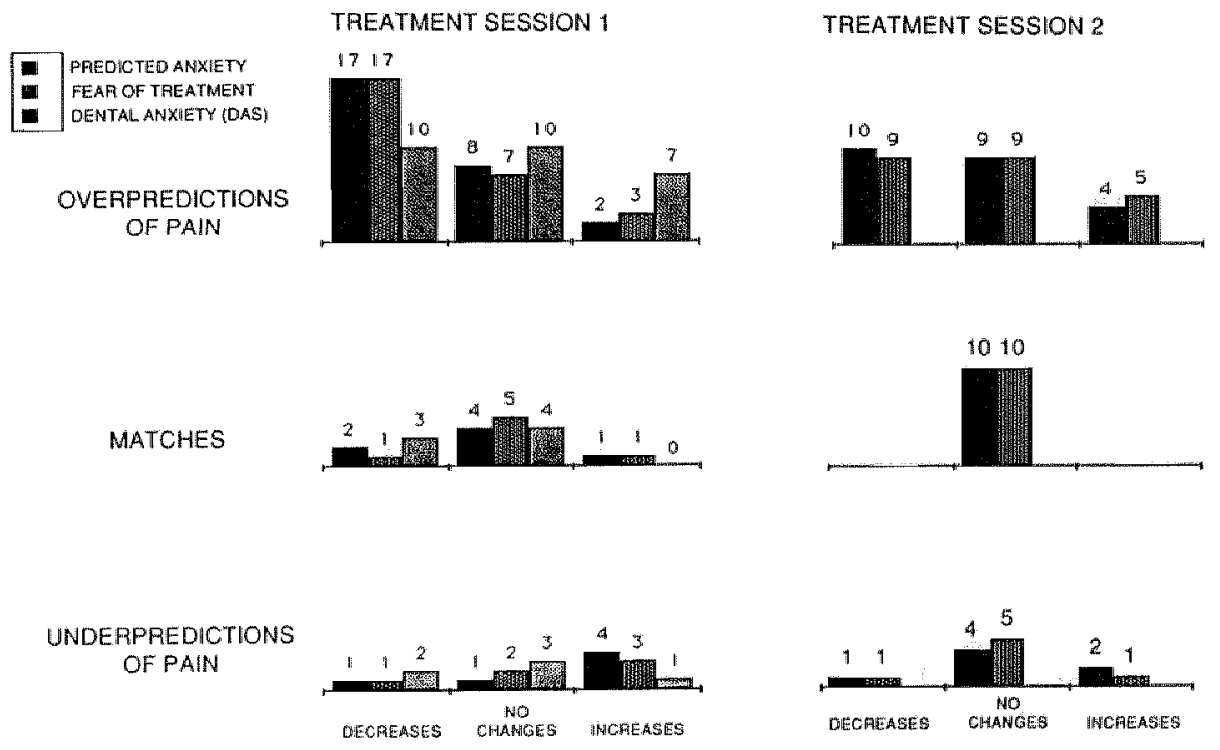

Figure 3. The relationship between inaccurate pain predictions and changes in predictions of anxiety $(p<0.01, p<0.02)$, changes in the score on the Dental Anxiety Scale (DAS) (n.s.), and fear of the next treatment $(p=0.02, p=0.02)$. Inaccurate pain predictions were related to specific 'state' measurements of anxiety, but were not related to the more general 'trait' of Dental Anxiety. 
A more specific (state) measurement of dental fear was obtained by VASs on fear of coming treatment, completed just before and after the two treatment sessions. Overpredicted pain at treatment 1 was related to decreases in fear and underpredicted pain (slightly) to increases in fear $\left(x^{2}(4)=11.72, p=0.02\right)$. At the second treatment changes in fear from before to immediately after treatment were less influenced by mismatches of pain predictions: most fear scores remained constant, but there was a tendency for overpredicted pain to be followed by reduction of fear $\left(\chi^{2}(4)=11.35, p=0.02\right.$ ).

Thus, the evidence for hypothesis 3 was mixed: whereas dental fear specifically related to a concrete treatment was influenced by mismatches of pain predictions, general dental anxiety (DAS) was not. Moreover, the effect appears to be more noticeable when there really is a next treatment than when the measurement pertains to a hypothetical later treatment (as after treatment 2).

The relationship between dental anxiety, pain predictions and experienced pain (hypotheses 4-7)

The topics that were feared most frequently by the $S s$ concerned issues related to pain (painful treatment, drilling and injections) and unpredictability of what might happen during treatment (see table 1).

Table 1. Frequency of feared topics

\begin{tabular}{lcc} 
Toplc & $N$ & $\%$ \\
\hline painful treatment & 17 & 42.5 \\
drilling & 17 & 42.5 \\
ignorance of what will happen & 13 & 32.5 \\
injections & 11 & 27.5 \\
extractions & 7 & 17.5 \\
rude dentist & 7 & 17.5 \\
general fear of doctors & 6 & 15.0 \\
shame about condition of teeth & 5 & 12.5 \\
stories from others & 4 & 10.0 \\
instruments & 3 & 7.5 \\
faulty treatment & 1 & 2.5 \\
other topics & 5 & 12.5 \\
\hline
\end{tabular}

Note $n=40$

This is in line with previous findings reported in the literature: fearful dental patients generally fear (unpredictably intense) pain due to treatment.

The $40 \mathrm{Ss}$ were divided into two groups by means of a median split on the Dental Anxiety Scale (DAS) of the first treatment. The median was 9. Ss with a DAS score $>9$ were assigned to the High Anxiety group (HA, $n=19$ ), Ss with DAS score $\leq 9$ to the Low Anxiety group (LA, $n=21$ ), $15 \mathrm{LA}$ and $16 \mathrm{HA} S \mathrm{~s}$ were contacted for the follow-up. Predicted, experienced and remembered pain of both groups are depicted in figure 4, means and $t$-tests are shown in table 2.

As will be clear from figure 4 , HA Ss expected to experience significantly more pain than the LA Ss at the first treatment, immediately after the first treatment, as well as before the second treatment. Immediately after the second treatment there seems to be a temporary decrease in pain predictions in the HA group, which is $n . s$. , however $(t(18)=$ 
1.55, $p=0.070,1$-tailed), and their predictions are still significantly higher than those of the LA group. At the follow-up, predictions are again higher.

The HA group significantly overpredicted pain at both treatment sessions, whereas Ss in the LA group were quite accurate in their pain predictions. The between group effect was significant at both treatments. Means and t-tests are shown in table 3 .

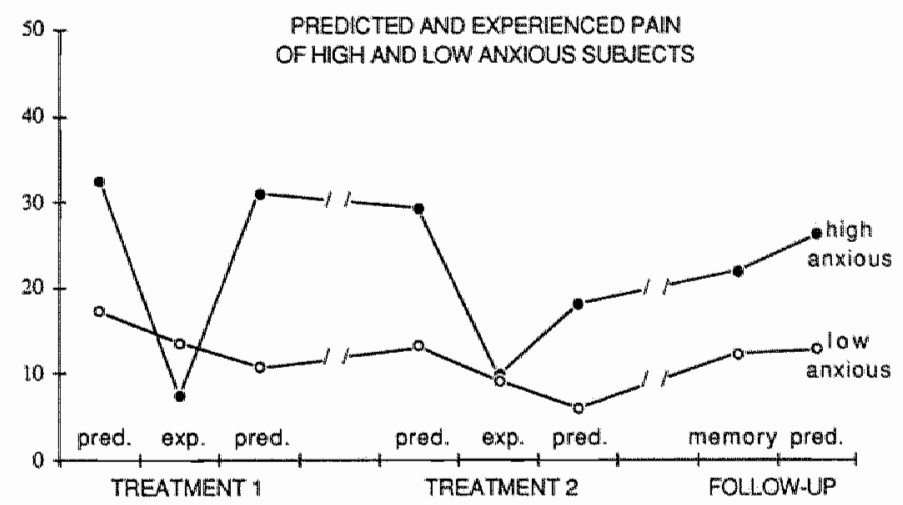

Figure 4. Predicted, experienced and remembered pain of low anxiety (LA) and high anxiety (HA) Ss. Whereas HA Ss did not experience more pain, they strongly overpredicted pain. During the treatment period they gradually became more accurate, but at the follow-up there was a return towards the original prediction level.

Table 2. Predicted and experienced pain of low and high anxious groups

\begin{tabular}{lrrrr}
\hline Measurement & $L A$ & $H A$ & $t$ & $p$ \\
\hline treatment 1 & & & & \\
prediction before & 17.3 & 32.5 & 1.84 & 0.039 \\
experience during & 13.6 & 7.4 & -1.08 & 0.855 \\
mean mismatch & 3.7 & 25.1 & 2.53 & 0.008 \\
prediction after & 10.8 & 31.0 & 2.23 & 0.018 \\
& & & & \\
treatment 2 & & & & \\
prediction before & 13.3 & 29.3 & 1.76 & 0.046 \\
experience during & 9.2 & 9.9 & 0.18 & 0.428 \\
mean mismatch & 4.1 & 19.3 & 1.94 & 0.033 \\
prediction after & 6.0 & 18.2 & 1.99 & 0.030 \\
& & & & \\
follow-up (5 months) & & & & \\
remembered experience & 12.4 & 22.0 & 1.68 & 0.053 \\
prediction & 13.0 & 26.4 & 1.98 & 0.029 \\
\hline
\end{tabular}

Note. All significance levels are one-tailed, testing the hypothesis HA $>$ LA. Since most wariances differed significantly, separate variance estimates were employed. 'Prediction after' means the pain prediction $S$ s made after the treatment session. These predictions concerned the following (hypothetical) treatment session. The "prediction' at follow-up concerned a hypothetical similar treatment. 
Table 3. Size of mean inaccuracy of pain predictions of HA and LA groups

\begin{tabular}{|c|c|c|c|c|c|c|}
\hline \multirow[t]{2}{*}{ Difference } & \multirow[t]{2}{*}{ group } & \multirow{2}{*}{$\begin{array}{l}\text { size of } \\
\text { inaccuracy }\end{array}$} & \multicolumn{2}{|c|}{ within effect } & \multicolumn{2}{|c|}{ between effect } \\
\hline & & & $t$ & $p$ & $i$ & $p$ \\
\hline $\begin{array}{l}\text { treatment I } \\
\text { pred - exp (1) }\end{array}$ & $\begin{array}{l}\text { LA } \\
\text { HA }\end{array}$ & $\begin{array}{r}3.7 \\
25.1\end{array}$ & $\begin{array}{l}0.73 \\
3.65\end{array}$ & $\begin{array}{l}0.475 \\
0.002\end{array}$ & 2.53 & 0.008 \\
\hline $\begin{array}{l}\text { treatment } 2 \\
\text { pred - } \exp (2)\end{array}$ & $\begin{array}{l}\text { LA } \\
\text { HA }\end{array}$ & $\begin{array}{r}4.1 \\
19.3\end{array}$ & $\begin{array}{l}1.68 \\
2.59\end{array}$ & $\begin{array}{l}0.109 \\
0.019\end{array}$ & 1.94 & 0.025 \\
\hline $\begin{array}{l}\text { follow-up } \\
\text { rem - exp (2) }\end{array}$ & $\begin{array}{l}\text { LA } \\
\text { HA }\end{array}$ & $\begin{array}{r}4.7 \\
11.4\end{array}$ & $\begin{array}{l}1.14 \\
2.68\end{array}$ & $\begin{array}{l}0.275 \\
0.017\end{array}$ & 1.12 & 0.135 \\
\hline pred $=\exp (1+2)$ & $\begin{array}{l}\text { LA } \\
\text { HA }\end{array}$ & $\begin{array}{r}1.5 \\
17.0\end{array}$ & $\begin{array}{l}0.30 \\
3.79\end{array}$ & $\begin{array}{l}0.767 \\
0.002\end{array}$ & 2.35 & 0.013 \\
\hline
\end{tabular}

Note. pred $=$ predicted pain; $\exp (\mathbb{1})=$ pain experienced at treatment 1 ; exp $(2)=$ pain experienced at treatment 2 ; exp $(1+2)=$ mean pain experienced at treatments 1 \& 2 ; rem $=$ remembered pain of second treatment. Between effects are tested one-tailed (hypothesis HA > LA).

Summarizing, it may be stated that anxious dental patients appear to have a chronic teridency to expect more pain than fearless patients, and they tend to overestimate this pain. Hypotheses 4 \& 5 are supported.

The decrease in pain predictions in the anxious group after the second treatment is in line with hypothesis 6: anxious dental patients can become more accurate in their predictions, but this takes several disconfirmations. Without further experiences, however, predictions will be heightened again, which is shown by the results of the follow-up.

Contrary to hypothesis 7 , dental anxiety is not associated with higher pain experiences: it will be clear from figure 4 and table 2, that the differences between the HA and LA groups as to experienced pain are n.s.

Dental anxiety was, however, associated with experiences of more intense anxiety during the first $(t(38)=3.23, p=0.003)$ and the second treatment $(r(38)=2.09, p=0.04)$. Predictions and experiences of anxiety during treatment are depicted in figure 5. As will be clear from figure 5 , both groups overpredict the anxiety they will experience during the first treatment (LA: $t(20)=2.15, p=0.04$; HA: $t(18)=2.22, p=0.04$ ), but the overprediction is not significantly larger in the HA group $(t(38)=-1.00, p=0.33)$. At the second treatment, the LA group has become quite accurate in predicting anxiety (difference n.s.), whereas the anxious group still overpredicts (between effect: $t(38)=2.67, p=0.01$ ).

\section{Memory and schemas of pain (hypotheses 8 \& 9)}

Memories of pain experienced during the second treatment were assessed 5 months after this treatment. Remembered pain was generally higher than experienced pain $(t(30)=2.73, p=0.01)$, but lower than pain predicted at treatment $1(t(30)=-2.43$, $p=0.02)$. Although the anxious $S \mathrm{~s}$ remembered significantly more pain than they actually experienced, and low anxiety $S$ s remembered there pain quite accurately, the between 


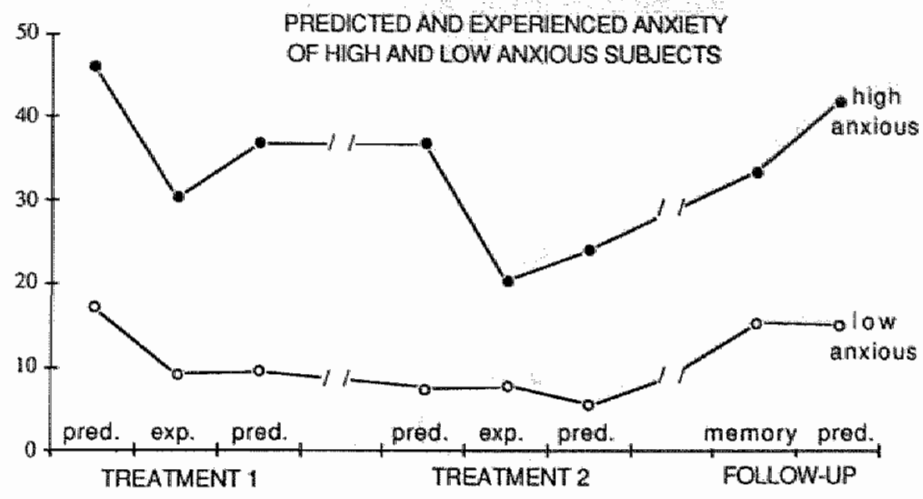

Figure 5. Predicted, experienced and remembered anxiety of low anxiety (LA) and high anxiety (HA) Ss. In contrast with experienced pain, HA Ss do experience more anxiety during treatment. Both groups overpredict at the first treatment. Only the LA group has become accurate at the second treatment.

effect was h.s. (see table 3) with respect to inaccuracy. However, a direct comparison between the memories of pain was significant $(p=0.05$, table 2$)$. It can be concluded that memories of pain were influenced both by experienced pain $(r=0.39, p=0.03)$ and by original predictions of pain $\left(r=0.66, p<10^{33}\right)$. Regression anallysis showed that these two factors have independent contributions. Although remembered pain is generally lower than originally predicted pain, the differences between $S$ s as to memories of pain are influenced more by the differences between $S$ s as to original pain predictions than by the pain that is actually experienced.

The same seems to be true of predicted pain assessed at the follow-up. At the followup, the predictions were generally lower than before the first treatment $(u(30)=-2.03$, $p=0.05$ ), but interindividual differences were still strongly related to the initial predictions $\left(r=0.76, p<10^{-3}\right)$.

Ss with high dental anxiety had somewhat more painful memories of the most painful treatment ever experienced than low anxiety $S s(t(27)=1.78, p=0.07$, one-tailed).

Summarizing, it can be stated that hypothesis 8 is supported: 5 months after the treatment sessions, memories and new predictions of pain appear to be influenced more by the original pain predictions than by the pain experiences. It must be acknowledged, however, that the experiences did have some influence, especially on memories. The higher pain predictions and memories of the HA group appear to be related to more painful memories of the most painful treatment, which is in agreement with hypothesis 9.

\section{Further exploration of change processes}

The processes underlying changes in pain prediction and in dental anxiety after overpredicted pain were further explored by means of regression analyses. First, factors that might influence decreases in inaccurately high pain predictions were investigated. Ss who made an overprediction or a match were selected. Change in pain prediction was analyzed as dependent variable in forward and backward regression analyses in which experienced pain, anxiety and aversiveness, discrepancies of expectations and the DAS score were predictors. Since initial high predictions allow of larger decreases than low predictions, 
Table 4. Factors that influence changes in pain prediction after overpredicted pain

\begin{tabular}{|c|c|c|c|c|}
\hline Dependent variable & predictor & $\beta$ & $t$ & $p$ \\
\hline \multicolumn{5}{|l|}{ predlcted pain } \\
\hline 1. change in predicted pain & predicted pain & 0.06 & 0.22 & 0.83 \\
\hline \multirow[t]{4}{*}{ measured after treatment 1} & experienced pain & 0.89 & 3.19 & 0.003 \\
\hline & dental anxiety DAS & 0.45 & 2.81 & 0.009 \\
\hline & experienced anxiety & 0.37 & 2.45 & 0.021 \\
\hline & $\mathrm{R}=0.86, \mathrm{~F}(4,29)=20.0, \mathrm{p}<10^{-4}$ & & & \\
\hline \multirow{3}{*}{$\begin{array}{l}\text { 2. change in predicted pain } \\
\text { measured before treatment } 2\end{array}$} & predicted pain & -0.64 & -4.94 & $<10^{-4}$ \\
\hline & experienced anxiety & 0.65 & 4.97 & $<10^{-4}$ \\
\hline & $\mathrm{R}=0.73, \mathrm{~F}(2,31)=18.2, \mathrm{p}<10^{-4}$ & & & \\
\hline \multirow{3}{*}{$\begin{array}{l}\text { 3. change in predicted pain } \\
\text { after treatment } 2\end{array}$} & predicted pain & -0.18 & -0.63 & 0.53 \\
\hline & experienced pain & 1.04 & 6.22 & $<10^{4}$ \\
\hline & $\mathrm{R}=0.82, \mathrm{~F}(3,29)=28.7, \mathrm{p}<10^{-4}$ & -0.33 & -1.99 & 0.056 \\
\hline
\end{tabular}

Note. Regression analyses were performed forwards and backwards and yielded almost identical results. Results of forward analyses are shown here. The initial level of pain prediction was controlled for (indicated by 'predicted pain"), since higher initial levels allow of larger decreases. Standardized $\mathrm{B}$ coefficients are given.

the initial prediction level was controlled for. The results are summarized in table 4 . These results indicate that decreases in pain predictions are larger when the experienced pain was low, which is not surprising. The effect of experienced anxiety is interesting: the lower the level of experienced anxiety is, the larger the reductions in pain predictions are. High dental ('trait") anxiety had a negative effect on reductions after the first treatment, but did not have this effect after the second treatment.

Comparable results are found when factors that influence decreases in fear of the next treatment after overpredicted pain are investigated (see table 5). Regression analyses, controlling for the initial level of fear, also show experienced pain and experienced anxiety as important predictors. Thus, experienced pain (lower than expected), as well as experienced anxiety, strongly influences the processes by which reduction of overestimation of pain and reduction of fear are brought about.

\section{DISCUSSION}

The first aim of the present study was to test the hypothesis that inaccurate pain and anxiety predictions are followed by changes in predictions in a way similar to what has been observed in the laboratory. The results clearly support this hypothesis. The deviations from the match/mismatch model observed after underpredicted pain and anxiety at the second treatment may be attributed to the absence of a real later treatment or to a chronic tendency to underpredict of some Ss. Inspection of individual data showed that some fearless $S s$ strongly underpredicted pain at both treatment sessions, but did not show any, or only minor, adjustment of prediction. It seems that the opposite of the chronic tendency to overpredict that is shown by the anxious $S$ s is formed by the tendency to underestimate that is shown by some fearless, almost overbold, $S \mathbf{s}$. Both groups seem to differ equally 
Table 5. Factors that infuence decreases in fear of treatment after overpredicted pain

Dependent variable

predictor

p

$t$

$p$

fear of treatment

1. change in fear of treatment

measured after treatment 1

$\begin{array}{lrrr}\text { initial fear } & -1.04 & -10.52 & <10^{4} \\ \text { experienced anxiety } & 0.57 & 4.44 & <10^{-4} \\ \text { experienced pain } & 0.29 & 3.64 & 0.001 \\ \text { dental anxiety DAS } & 0.31 & 2.43 & 0.022 \\ \mathrm{R}=0.90, \mathrm{~F}(4,29)=32.2, \mathrm{p}<10^{-4} & & & \\ \text { initial fear } & & & \\ \text { experienced anxiety } & -1.08 & -5.75 & <10^{-4} \\ \text { experienced pain } & 0.51 & 3.69 & 0.001 \\ \text { owerestimation of pain } & -0.35 & -2.08 & 0.047 \\ \mathrm{R}=0.78, \mathrm{~F}(4,29)=12.1, \mathrm{p}<10^{-4} & & & \\ & & & \\ \text { initial fear } & -0.66 & -3.09 & <10^{-3} \\ \text { experienced anxiety } & 0.42 & 2.44 & 0.021 \\ \mathrm{R}=0.59, \mathrm{~F}(2,29)=7.83, \mathrm{p}=0.002 & & & \end{array}$

2. change in fear of treatment measured before treatment 2

3. change in fear of treatment measured after treatment 2

$$
R=0.59, F(2,29)=7.83, p=0.002
$$

Note. Regression analyses were performed forwards and backwards and yielded identical results for $1 \& 3$. For 2 , almost identical results were obtained (results of the backward analysis are shown). The initial level of fear was controlled for, since higher initial levels allow of larger decreases. Standardized $\&$ coefficients are given.

from the moderately fearful $S$ s in the way in which they adhere to an inaccurate schema. Underpredicting $S$ s were in the minority, however. Most $S$ s overpredjcted the aversive aspects of dental treatment.

The second aim pertained to the relationship between inaccurate pain prediction and changes in dental anxiety. Whereas clear results have been obtained in the laboratory (underpredicted pain leads to increased fear of pain, owerpredicted pain to decreased fear; Arntz \& van den Hout, 1988; Amtz \& Lousberg, 1990), results in the ecologically valid situation of dental treatment are not very straightforward. Measurements of fear and predicted anxiety of a concrete treatment clearly validated the laboratory findings, but changes in the more general "trait" of dental anxiety (DAS) were not significantly related to matches/mismatches of pain predictions. Trait-like factors that are not easily influenced appear to play an important part outside the laboratory setting.

This trait-like factor connected with dental anxiety, measured by the DAS, was indeed a strong moderator. As was hypothesized, highly anxious $S$ s strongly overpredicted experienced pain and anxiety due to treatment, whereas low anxiety $S$ s were more accurate. The general conclusion can be drawn that although highly anxious $S$ s show a chronic tendency to overpredict, they do learn from experience and can become more accurate. They need quite a number of disconfirmative experiences, however, and if the number is not large enough, and experiences do not follow each other closely, the old ideas will appear to become restored in time (which is shown by the follow-up measurement), and although they are influenced by experience, they are determined most strongly by the original conceptions (see also Kent, 1985).

Although highly anxious $S$ s expect more pain and feel more anxious during treatment, they do not experience more pain. This finding is at odds with some conceptions about the relationship between anxiety and pain (e.g. see Chapman \& Turner, 1986). There does not appear to be a direct relationship between anxiety and experienced pain. Why, then, does 
the problem of dental anxiety and inaccurate pain expectations continue to exist, despite disconfirmations? Several explanations can be derived from the literature on the subject. First, it has been argued that dental treatments generally do not follow each other closely, which makes habituation of the anxiety response difficult (Lindsay, 1983). However, this does not explain why patients continue to fear things that do not happen (it only explains why a common method of fear reduction does not apply to the average dental treatment). Second, it has been proposed that highly anxious $\$$ s attribute disconfirming experiences to the atypicality of the treatment (Wardle, 1984; Kent, 1986). Thus, it is suggested that disconfirming experiences are not incorporated because they do not fit into the domain of situations to which the dominant schema of the $S$ applies. The problem of this explanation is, however, that it is not clear whether this attribution is really the cause of the continuation of the old ideas, or whether it is a post-hoc explanation used by the patient to reduce his cognitive dissonance (discrepancy between expected and experienced pain) elicited by the questions of the investigator. It should be noted that typicality was assessed post-hoc in the study by Kent. A different timing of the assessment of the typicality may clarify this issue. The third explanation proposes that the availability of more painful experiences in human memory is disproportionally high and therefore dominates the generation of expectations (Lindsay, 1983). However, it is not clear whether anxious $S$ s have really experienced more extremely painful treatments (although in the present study they had more painful memories of the most painful treatment, but these may be inaccurate).

The present study confirms the general idea that the lack of a close succession of treatment sessions is an important factor in the maintenance of dental fear, but this time the emphasis is not on habituation but on correction of inaccurate cognitions. Repeated processing of new information without allowing time to let the old schema recover appears to be necessary for inducing change. Another interesting factor was revealed by the analysis of the change processes immediately after disconfirming experiences. Two variables consistently appeared to be important: (1) the level of experienced pain (the lower, the more reduction of fear and of inaccuracy of predictions), and (2) the level of experienced anxiety (the higher, the less predictions and fear are reduced). The last factor has clinical and theoretical significance.

Clinically, it suggests that assisting $S s$ in reducing their level of experienced anxiety may help them to reduce their fear and inaccurate expectations. Procedural (information, predictability, controllability, timing) as well as interpersonal (empathic understanding, patience, humour) factors have been proposed as helpful (e.g. Lindsay, 1983). The socialemotional climate is probably an important factor (and it is often neglected by dentists) for the processing of new experiences, as it is in psychotherapy (cf. Rachman, 1980).

Theoretically, the influence of experienced anxiety may seem strange: it is not immediately clear why overprediction is reduced less when the $S$ felt anxious during treatment (other factors being constant). Several explanations can be offered. First, Eysenck's incubation theory states that the conditioned response (anxiety) elicited by the conditioned stimulus (treatment) may acquire a negative quality in itself and become a reinforcer of the CS-CR relationship (Eysenck, 1968). Similarly, Beck states that the experienced anxiety functions in a positive feedback loop, reinforcing inaccurate expectations (Beck, Emery \& Greenberg, 1985). Another explanation may be that reality testing is more difficult when $S \mathrm{~s}$ are anxious, either because the testing process itself is disturbed (which makes it difficult to test expectations against facts), or because the anxiety felt is confused with the objective danger that is feared, but does not exist in reality. These are interesting hypotheses that call for further investigation which may clarify the puzzle why such problems can continue to exist despite disconfirmations. It may also be interesting to 
investigate whether negative emotions play a comparable role in the maintenance of chronic clinical pain problems.

Whatever the underlying mechanism may be, the present study clarifies that dental anxiety and inaccurate expectations do not continue because anxiety causes increased pain experiences, but because experienced anxiety interferes with the change processes of subjective schemas. Since fearful $S s$ experience more anxiety during treatment, it is more difficult for them to correct their inaccurate expectations. 



\section{CHAPTER 3. \\ EFFECTS OF UNPREDICTABLE INCREASES \\ IN PAIN INTENSITY}

Chapter 3 addresses the effects of unpredictable increases in pain intensity. In the four studies reported in this chapter, the pain intensity is objectively increased at one or several trials without the $S$ 's knowledge. Effects of these unpredicted increases in pain intensity on habituation, fear of pain and pain predictions are investigated. The general theory that is tested is that unpredictable increases in pain intensity have a number of negative effects, notably on subjective fear of pain, on fear behaviour (escape and avoidance), on physiological fear responses, on pain predictions, and on pain responses.

In these studies two forms of expectation are manipulated together: when the increase in intensity of a stimulus is unsignalled, most $S$ s underpredict the stimulus (the pain level they consciously expect is lower than the pain experience), but the increased stimulus also deviates from a regular pattern, and lower levels of the central nervous system (CNS), not involving conscious information processing, might be sensitive for such sudden deviations. Therefore, as was argued in section 2.3, the results of this chapter should be interpreted as related to both levels (the higher, conscious level, and the lower level also sensitive for deviations from regularity).

In section 3.1 the differential effects of an early $v s$ a late unpredicted increase in pain intensity on physiological and subjective habituation, fear of pain and pain predictions are tested. It is hypothesized that a late heightened pain stimulus has relatively stronger effects than an early heightened stimulus, because the stimulus representation is less developed in the early phase of the sequence.

In section 3.2 the hypothesis is tested that unexpected increase in pain intensity is related to escape behaviour, rather than the objective intensity level of the painful stimulus.

In section 3.3 the hypothesis is tested that an unpredicted increase in pain is related to subsequent avoidance behaviour.

In section 3.4 the relationship between unpredictable increases in pain intensity and physiological and subjective indicators of fear is further explored. In this study, the hypothesis is tested that subjective fear and physiological reactions to a warning signal are more profound, and more resembling phobic anticipatory reactions, in a condition with unpredictable increases to a high pain intensity level, than in a condition with a constant (therefore predictable) high level of pain intensity. 


\section{SECTION 3.1. THE EFFECTS OF UNDERESTIMATED PAIN AND THEIR RELATIONSHIP TO HABITUATION ${ }^{\wedge}$}

\section{SUMMARY}

The intensity of a painful stimulus was experimentally mamipulated in order to induce underpredictions of pain. The experiment aimed at (1) replicating previous findings on the effects of underpredicted pain and (2) investigating the relationship between underpredicted pain and habituation. Most previous findings were replicated: underpredictions of pain were followed by raised expectations and increased fear of pain. In addition, the underprediction had long-term effects on fear of pain, uncertainty about predictions, and expected aversiveness of the painful stimulus. In contrast to previous findings and to Rachman's match/mismatch model, it was found that underpredicted pain can have dishabituating effects. It is theorized that inaccurately predicted pain can cause dishabituation, depending on the extent of the underprediction and on the subjective certainty of the prediction. Theoretical and clinical implications are discussed.

\section{INTRODUCTION}

It has recently been shown that, in addition to the unpredictable occurrence of aversive events, the unpredictable intensity of aversive events is also an important factor in the understanding of neurotic problems (Arntz \& van den Hout, 1988; Rachman \& Bichard, 1988). A distinction should be made between immediate and long-term consequences of incorrectly predicted intensity. The most important immediate consequence of a mismatch between expected and experienced intensity of fearful or painful events is that the expectation for the next experience is adjusted in the direction of this last experience. When the event is more aversive than expected (underprediction), expectations for the next event will probably be heightened; when the event is less aversive than expected (overprediction), expectations will be probably lowered; and when the expectation is accurate, expectations generally remain constant (Rachman \& Levitt, 1985; Rachman \& Lopatka, 1986a, b; Rachman \& Lopatka, 1988; Arntz \& van den Hout, 1988). It has been shown that the last experience can be very important in forming the next prediction (Arntz \& van den Hout, 1988).

There appear to be some asymmetries, however, in the effects of underpredictions and overpredictions. Underpredictions of fear and pain seem to have stronger effects on subsequent predictions than do overpredictions (cf. Arntz \& van den Hout, 1988; Rachman \& Bichard, 1988; Rachman \& Lopatka, 1986b). Furthermore, overprediction (which may result from an underprediction) can be relatively long-lasting, needing more disconfirmations before it becomes accurate. Clinical problems seem to be characterized by a chronic tendency to overpredict fear (Rachman \& Bichard, 1988), pain (Kleinknecht \& Bernstein, 1978; Wardle, 1983; Kent, 1984; Arntz, Heymans \& van Eck, 1989) or aversive events

'Reprinted with permission from Behaviour Research and Therapy, 28, Arntz, A. \& Lousberg, R., The effects of underestimated pain and their relationship to habituation, 1990, Pergamon Press PLC. 
in general (Beck, 1976; Butler \& Matthews, 1983). In the only laboratory demonstration of experimentally induced underprediction that we know of, it was found that the (relatively mild) underprediction induced by the experimenters was followed by a tendency to overpredict that needed a mean number of two trials to return to an adequate level (Arntz \& van den Hout, 1988). Rachman and co-workers hypothesize that in real life overprediction is related to avoidlance behaviour, which reduces the opportunities for disconfirmation (Rachman \& Bichard, 1988). Thus, in general people seem to prefer to be on the safe side after an underpredicted aversive event. In addition, there is evidence that an underprediction delays the decrease in fear for the event and that $S$ s remain uncertain about what to expect for a relatively longer time (Arntz \& van den Hout, 1988). These immediate and long-term effects of mismatches between expectations and experiences seem to be relatively clear. However, some issues are not yet understood.

The first issue relates to avoidance and escape. Although there appears to be ample evidence that overprediction and avoidance behaviour are correlated in clinical problems (e.g. Kleinknecht \& Bernstein, 1978; Liddell \& May, 1984; Philips, 1987), it is hardly clear whether avoidance behaviour is a direct consequence of underprediction. Rachman \& Lopatka (1986b) found that after underpredicted fear, snake phobics showed slower approach and more hesitations in the next trial of exposure to a snake. Arntz and van den Hout (1988) found that $S$ s tended to shorten the time they took for indicating that they were ready to receive the next painful shock, which may tentatively be interpreted as an escape tendency from the experiment as a whole. The very important issue of the relationship between underprediction and subsequent escape/avoidance requires more study.

The second issue pertains to the relationship between discrepancies between expectancies and experiences on the one hand, and habituation on the other hand. Theoretically speaking, it can be hypothesized that adequate expectancies further the habituation process. Modern learning theories stress the importance of expectancies and the so-called 2 -stage theories of habituation postulate that habituation is an active process of inhibiting (suppressing) the responses elicited by the stimulus. This inhibition is supposed to be based on a 'neurological model' (or a 'memory model') of the stimulus, in other words on the expectation of the stimulus (Gray, 1975; Wagner, 1979; Mackintosh, 1987). It will be clear, then, that discrepancies between expectation and experience must have effects on this suppression process: there will only be inhibition if the stimulus matches its neurological model (Gray, 1975). In addition, the biological function of signalling discrepancies seems to be clear: discrepant experiences convey more information than experiences that are identical to expectation, which seems to apply especially to underpredictions because of their greater significance for the integrity of the organism. For these reasons, it can be hypothesized that mismatches, and especially underpredictions, have dishabituating effects: the responses to the stimulus are supposed to be temporarily stronger. In fact, intensity is one of the dimensions, summarized by Gray (1975) in his review of the literature on the $S$, on which discrepancies between expectancy and experience can have habituation disturbing effects (see also Sokolov, 1960). There does not seem to be much empirical evidence from match/mismatch studies for this hypothesis, however. In none of the studies by Rachman and co-workers was any effect of mismatches on the habituation process found. Arntz and van den Hout (1988) could not find any dishabituating effects of underpredictions of painful electric shocks, either.

These studies leave us with a paradoxical situation. On the one hand, mismatches have clear cognitive (expectancy, certainty), emotional (anticipation anxiety) and behavioural (speed, hesitations, perhaps avoidance) effects; but on the other hand, there seems to be another process that controls habituation which is unconnected with it (cf. Rachman \& 
Lopatka, 1986a).

These findings are even more astonishing, as this dissociation cannot be attributed to different levels of the nervous system that would control these processes. It will be clear that the neocortex and areas that have a memory function (structures in the limbic system) have a function in forming expectancies (at least as expressed on rating scales). The same areas, however, appear to play an essential part in habituation processes (at least in more highly developed species, see Gray, 1975). Thus, it seems that this apparent dissociation cannot be explained by the involvement of different nervous systems in the two processes. Some findings in the study by Amtz and van den Hout (1988) may, however, indicate a factor that plays a part in this. In this study it was found that high certainty about the intensity expected was related to smaller effects of mismatches, and, in case of a match, to stronger habituation. Thus, there is some evidence that the certainty of expectancy is related to habituation, and that strong expectancies can protect the organism against the effects of discrepancies. This second finding, however, seems to be in contradiction with the modem learning theories mentioned above: deviations from strong expectancies give more information than deviations from weak expectancies, and should therefore have stronger effects. In a totally different context, this was, in fact, found: $S$ s who were very certain about their expected result on an IQ test got more upset when they leamed that their result (which was given to them by means of bogus feedback) differed markedly from what they expected, than uncertain Ss (Albersnagel, Arntz \& Gerlsma, 1986).

The relationship between habituation and mismatches is unclear, but is probably related to (a) the certainty of the expectation and (b) the magnitude of the discrepancy between expectation and experience. Since $S \mathrm{~s}$ become more certain about their expectations with repeated experience (see Arntz \& van den Hout, 1988), the effect of certainty can be investigated by comparing an experimentally induced late mismatch with a mismatch induced early in the series of trials. From the above discussion two mutually exclusive hypotheses follow: (a) high certainty reduces the dishabituating effect of underprediction or (b) high certainty increases the dishabituating effect.

The third issue pertains to the relationship between mismatches and various responses to the aversive stimulus. With respect to painful stimuli, pain-intensity, fear of painful shock and the time $S$ s took to indicate that they were ready for the next trial have been investigated (Arntz and van den Hout, 1988; Rachman \& Lopatka, 1988), but other responses are of interest too: the emotional experience of pain (which can be distinguished from the intensity experienced, cf. Gracely, 1979; Melzack, 1980) and physiological responses.

The main aims of the present study are the following:

1. To replicate the main findings of the study by Arntz and van den Hout (1988) on the effects of underpredicted pain and to generalize these findings to emotional and physiological pain responses.

2. To investigate the relationship between underprediction and habituation further, and more specifically the role of certainty about expectation in this.

Two groups of hypotheses are formulated according to these aims. The first group of hypotheses addresses the attempt at replication and includes hypotheses that follow directly from Rachman's match/mismatch model (hypotheses $1 \& 6$ ). The second group consists of new hypotheses regarding the effects of an underprediction, and its possible dishabituating effects when the $S$ is very certain about what to expect. 


\section{Underprediction}

1. Underpredicted pain will be followed by increases in predicted pain (which last for more than 1 trial).

2. Underpredicted pain will have a long-term negative influence on certainty of prediction, which will remain relatively lower.

3. Underpredicted pain will have a long-term negative influence on fear of painful shock.

4. After an underprediction Ss will indicate that they are ready for the next trial more quickly (escape).

Habituation and related issues

5. Experienced pain and physiological responses will decrease over time (habituation).

6. Accuracy of predictions will increase with practice.

7. Certainty of prediction will increase over time.

8. Fear of shock will decrease over time.

New hypotheses

9 a. Late underpredicted pain will have smaller effects than early underpredicted pain.

b. Late underpredicted pain will have larger effects than early underpredicted pain.

10a. Underpredicted pain will not have any effect on habituation.

b. Underpredicted pain will have a dishabituating effect.

In order to compare the possible different effects of an underprediction in certain ws uncertain conditions, an underprediction was experimentally induced by a temporary increase of the shock level at the third trial in a series of 20 painful electric shocks in one experimental condition (early mismatch), and in the second experimental condition (late mismatch) an underpredietion was induced at the 13th trial. The third group (control group) did not receive any induced mismatches.

\section{METHOD}

\section{Subjects}

The Ss were 46 volunteers who participated for a renumeration. As a result of technical problems with the computer controlling the experiment, four $S$ s did not receive the planned protocol, and were excluded. Two of the remaining $42 \mathrm{Ss}$ were unemployed, and 40 were students. The average age was $21.05 \mathrm{yr}$ (range 18-25, $\mathrm{MD}=20, \mathrm{SD}=2.99$ ). There were 17 men and 25 women.

\section{Materials}

The materials included a personality questionnaire (ABV, De Wilde, 1983) with four subscales: neuroticism, psychosomatic complaints, extraversion and defensiveness. The Ss indicated their experienced as well as predicted pain-intensity and pain-aversiveness, on visual analogue scales (VAS), $10 \mathrm{~cm}$ horizontal lines ranging from 0 ("not at all painful", "not at all aversive") to 100 ("extremely painful", "extremely aversive"). The certainty 
about these predictions was also measured by means of VAS (" 0 , not certain at all $1^{\mathrm{m}}$ $" 100$, completely certain $"$ ), and so was fear of next shock ("0, not afraid at all" " 100 , extremely afraid").

\section{Physiological recording}

Skin conductance response (SCR) and skin conductance level (SCL) were measured by a Beckman Skin Conductance Coupler (type 9844), and the method of a constant voltage $(0.5 \mathrm{~V})$ was used. The coupler allowed for a maximum sensitivity of 0.05 micromho. Electrodes were attached to the medial phalanges of the second and third fingers of the non-dominant hand with adhesive collars. The highest response within $6 \mathrm{sec}$ after stimulus onset was measured.

Finger Pulse Volume (FPV) was monitored by means of a photocell (Beckman radial transducer 215560) placed on the tip of the fourth finger of the S's non-dominant hand. The transducer was connected to a Beckman Voltage/Pressure/ Volume coupler (type 9853 A). The time constant was $0.1 \mathrm{sec}$.

The FPV-response was measured according to the following criteria by Siddle and Heron (1975): first, determine the mean pre-stimulus value during $12 \mathrm{sec}$ before stimulus onset (FPVpre). Second, determine the mean of the three smallest FPVs of 10 pulses after stimulus onset (FPVpost). Third, compute the difference between the response and prestimulus value in percentages: FPV $\%=100 \% \times$ (FPVpre-FPVpost)/FPVpre. Because of missing data, analyses were performed on the basis of the data of $33 \mathrm{Ss}$. Since the pretrial FPV level may influence the size of the response (a pretrial level of large pulses allows for a larger response than a level of small pulses) individual correction for pretrial level was executed by means of regression analysis: the final FPV\% response of a trial was defined as the FPV\% response defined above minus the contribution ( $\beta_{1} \times$ pretrial level) to the response by the prestimulus level: corrected FPV $\%=$ FPV $\%-\left(B_{1} \times\right.$ pretrial level $)=B_{0}+$ error.

Heart rate (HR) was recorded from electrodes arranged according to a lead II placement (Stern, Ray and Davis, 1980) and connected to a Beckman Voltage/Pressure/Coupler (type $9853 \mathrm{~A}$ ). Because of the noise generated by the electric shock, beats during stimulation could not be reliably detected. Because of this, acceleration due to shock could not be measured. Analyses were restricted to the mean $12 \mathrm{sec}$ pre-stimulus (anticipation) period.

For measuring $\mathrm{HR}$ and $\mathrm{SCR}$, Beckman $\mathrm{Ag}-\mathrm{AgCl}$-electrodes $(8 \mathrm{~mm}$ diameter) filled with Hewlett Packard Redux paste were used.

\section{Apparatus}

The shocks had a duration of $6 \mathrm{sec}$ and were delivered by a Siemens Neuroton 627 , passed for use with patients. Shocks were given via a concentric shock electrode, placed on the $S^{\prime \prime}$ s right ankle (cf. Tursky 1974). A microcomputer PDM-Minc 11 controlled onset of the electric stimuli, inter-trial intervals and registration. The microcomputer was also used for on-line processing of the cardiac inter-beat intervals.

\section{Procedure}

Ss were randomly assigned to the control group $(n=14)$, the 'early' experimental condition $(n=14)$ or the 'late' experimental condition $(n=14)$. After the $S \mathrm{~s}$ had been 
introduced to the laboratory and informed consent had been obtained, the electrodes (SCR and HR) were placed on the body. Then the shock level that was to be used was determined (the calibration phase). From $0.0 \mathrm{~mA}$ the level was raised in steps of $0.4 \mathrm{~mA}$, until the $S$ indicated that the shock became aversive. After that the $S$ was asked to rate its painfulness and aversiveness on the visual analogue scales. The shock-level was then raised further until both VAS scores exceeded $50 \mathrm{~mm}$. This level was used during the experiment. After this, the $S s$ were asked to complete the personality questionnaire. After completion, the $S s$ were given a loose-leaf file containing the analogue scalles. The file contained scales for a series of 100 trials. The $S s$ were instructed to complete 7 analogue scales after each shock, rating pain and aversiveness experienced, pain and aversiveness expected at the next shock, certainty of these expectations and fear of the next shock. Before the first shock, $S$ s rated only the scales that concerned this shock. The $S$ s were asked to indicate that they were ready to receive the next shock by pressing on a button after each trial. The time between the onset of the shock and the Ss's indication that they were ready was measured in seconds. $S s$ were neither given information about the induced underprediction and the number of shocks they would receive, nor about the time they were to wait between their indication that they were ready and the shock. Twenty unannounced shocks were given after a random waiting time (mean $30 \mathrm{sec}$, range 15-45 sec). $S s$ in the control group were given shocks of the level that was determined in the calibration phase (mean level $=5.36 \mathrm{~mA} ., \mathrm{SD}=3.11 \mathrm{~mA}$.). $S \mathrm{~s}$ in the early experimental group were also given shocks of the calibration level $(m e a n=3.91 \mathrm{~mA} ., S D=1.82 \mathrm{~mA}$.), except at the third trial. At this trial the intensity level was raised by $50 \%$ according the psychophysiologicall relationship

$$
\text { intensity } \sim(\text { current })^{2.1}
$$

(cf. Lindsay \& Norman, 1977). Note that a $50 \%$ increase in intensity is equal to a current that is 1.213 times stronger. From the fourth trial, the pre-test level was used again. Ss in the late experimental group were given shocks of the intensity of those of the calibration phase (mean $=4.16 \mathrm{~mA}, \mathrm{SD}=2.10 \mathrm{~mA}$.) except at the 13 th trial. As was the case in the early group, the intensity level was raised with $50 \%$. From the 14 th trial, the pre-test level was used again.

Note that 20 experienced pain and aversiveness scores, 20 time scores, 21 predicted pain and 21 certainty scores about this prediction, 21 predicted aversiveness and 21 related certainty scores, and 21 fear scores were obtained for each $S$.

\section{RESULTS}

The results of the replication hypotheses will be presented first, followed by the results pertinent to the new hypotheses. VAS-scores were analyzed by means of MANOVA for repeated measures along the lines of the approach described by O'Brien and Kister Kaiser (1985). The conditions were compared as to change scores representing hypothesized effects: early was contrasted with late + control, and late with early + control (as far as the early and control groups were NS different at the later trials; if there were significant differences, the late group would be contrasted with the control group only). Hypotheses concerning habituation and related issues were tested by comparing later trials with the average scores of the trials before the third shock. All hypotheses were tested on a 1-tailed basis, except for hypotheses 9(a) and (b). 
There were no significant pre-test differences between the three conditions as to age $(\mathrm{df}=2, F=0.88, p=0.42)$, sex $\left(\mathrm{df}=2, x^{2}=0.20, p=0.91\right)$, study $\left(\mathrm{df}=8, x^{2}=11.56, p=\right.$ 0.17 ), and shock level $(F(2)=1.44, p=0.25)$. The groups did not differ in personality characteristics as measured by the $\mathrm{ABV}$ (defensiveness: $F(2)=0.08, p=0.93$; somatization: $F(2)=0.42, p=0.66$; neuroticism: $F(2)=1.37, p=0.27$; extraversion: $F((2)=1.24$, $p=0.30)$ ).

\section{Was the induction successful?}

As can be seen in figure 1(a) and (b), subjective intensity and aversiveness scores increased significantly by the early (at trial 3 ) as well as by the late (at trial 13) experimentally increased pain stimulus (trial 3 vs 2 : intensity $t=1.85, p=0.036$; aversiveness $t=2.74, p=0.005$; trial 13 vs 12 : intensity $t=3.90, p<0.001$; aversiveness: $t=4.09, p<$ 0.001 ). The physiological responses (figure $1(\mathrm{c})$ and (d)) were also increased by the stronger pain stimulus at the 13th trial (SCR $t=3.17, p=0.002$; FPV $t=2.87, p=0.004$ ). The stronger pain stimulus at the third trial did increase the FPV response $t=1.66, p=$ $0.054)$ but the SCR was not significantly higher $(t=0.55, p=0.29)$. In short, it can be concluded that both experimentally-increased pain stimuli led to elevated subjective and physiological responses.

\section{Effects of underprediction on predictions (hypothesis 1)}

As shown in figure 2(a), there was a significant increase in predicted pain intensity after the early underprediction (as contrasted with the late + control group: trial 3 vs 2 : $t=1.85, p=0.001$ ). The scores of the fourth trial are no longer significantly higher (trial 4 vs 2 : $t=0.65, p=0.28$ ). However, long-term effects are found for predicted aversiveness (see figure 2(b)). After the early underprediction, the scores were significantly higher up to, and including, the 7 th trial (all $t s>1.61$, all $p s<0.057$ ).

Identical results were found after the late induced underprediction. The immediate increase in predicted intensity is significant when contrasted with the control group and early condition (trial 13 vs 12: $t=3.28, p=0.001$ ). There are, however, no long-term effects on the predicted intensity (trial 14 is 12: $t=1.23, p=0.133$ ). The results of the VAS for predicted aversiveness indicate that, in addition to the immediate effect, there were long-term effects as well: the 14 th prediction was still higher $(t=1.93, p=0.031)$ m

From these results it can be concluded that hypothesis 1 is confirmed. It was found that the inductions had long-term effects on predicted aversiveness, but not on predictions of intensity.

\section{Effect on certainty of underpredicted pain (hypothesis 2)}

As can be seen in figure 2 (c), the early experimental induction had neither immediate nor long-term effects on certainty about predicted pain (trial 3 vs $2: t=-0.05, p=0.48$; trial 4 vs $2: t=-0.22, p=0.41$ ).

As shown in figure 2(d), certainty of the aversiveness predictions was not influenced significantly by the early underprediction (trial 3 vs 2 : $t=-0.06, p=0.48$; trial 4 vs 2 : $t=$ $-0.35, p=0.36)$. The late induced underprediction had a very long and relatively strong effect on the certainty about predicted pain intensity. Up to and including the 20th trial (the end of the experiment), certainty about the predictions was significantly lower (all ts $<-1.89$, all $P_{s}<0.034$ ). Certainty-scores of the aversiveness predictions were significantly lower up to the 19 th trial (all ts $<-2.11$, all $P_{S}<0.021$ ) and still somewhat low at the 20th trial $(t=-1.41, p=0.084)$. 
Thus, the effects following the late underprediction confirm hypothesis 2 . However, the early underprediction was not followed by any effect on certainty.

(a)

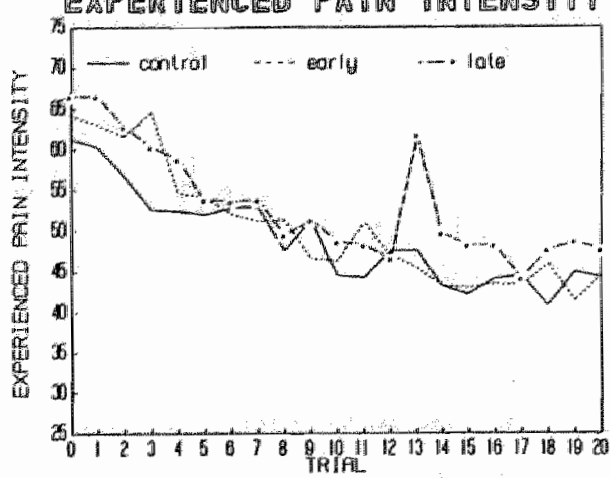

(c)

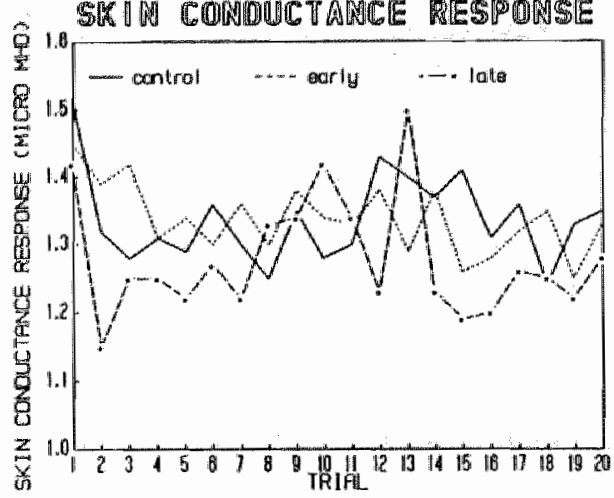

tho

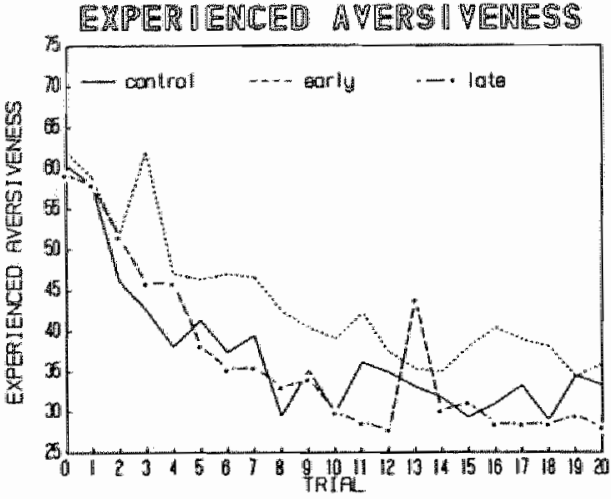

(d)

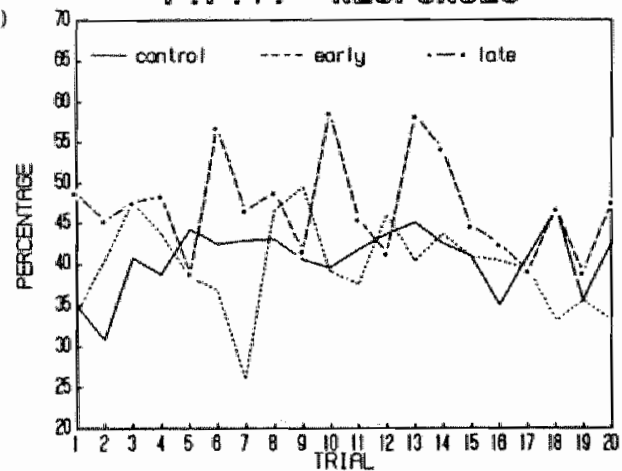

Figure 1. Experienced pain intensity (figure 1(a)), experienced pain aversiveness (figure 1(b), SCR's (figure 1(c)) and FPV responses (figure 1(d)) for the early, late and control conditions. Trial 0 indicates the calibration phase, in which shock level was determined. In the early condition the pain stimulus was temporarily increased at the third trial, in the late condition at the 13th trial, in order to induce underpredictions of pain.

\section{Prolonged fear of shock (hypothesis 3)}

The mean fear scores of each trial are shown in figure 3(a). After both experimentally induced underpredictions there were immediate and prolonged increases in fear scores. The immediate increase of fear after the early induction is significant (trial 3 vs 2 : $t=2.08, p=0.022$ ). The scores remain (marginally) significantly higher $u p$ to and including the 8th trial (all $t s>1.32$, all $P s<0.098$ ).

Inmediately after the late induction of underprediction there was a significant increase in fear of the next shock (trial 13 ws 12: $t=2.77, p=0.005$ ). There are marginally significant long-term effects up to and including the 16th trial (trial $14 \mathrm{vs} 12: t=1.52$, $p=0.068$; trial 15 w $12: t=1.67, p=0.052$ and trial 16 vs $12: t=1.28, p=0.10$ ).

Hypothesis 3 is confirmed: the experimental inductions seem to have delayed the reduction in fear of shock. The prolonged increases in fear scores after the early induction are especially remarkable. 
Effect on speed (hypothesis 4)

The average time that $S$ s used for completing the scales and for indicating that they were ready for the next trial are depicted in figure $3(\mathrm{~b})$. There is no indication that $S$ s took less time after the early underprediction (trial 3 is $2: t=-0.99, p=0.165$ ).

After the late induced underprediction there was, a marginally significant deceleration (trial 13 vs 12: $t=1.38, p=0.088$ ) which is inconsistent with the hypothesized acceleration. The effect of deceleration is also marginally significant at the 14th trial (trial 14 ws 12: $t=1.46, p=0.067$ ). Hypothesis 4 is rejected.

\section{Habituation and related issues}

Experienced pain and physiologicall responses will habituate (hypothesis 5)

The mean experienced intensity and aversiveness scores are depicted in figure 1( $a$ and $b$ ). From trial 4 onwards, there was a significant decrease of experienced pain (all trials: $F(2,39)>5.25$, all $\left.P_{s}<0.027\right)$. Experienced aversiveness was lower from the 3rd trial onwards (all trials: $F(2,39)>9.33$, all $P_{S}<0.02$ ).

SCRs did not show any habituation effect if the average SCR of trials 1 and 2 is used as a reference. This was caused by the (temporarily) strong decrease at the second trial in the late condition (see figure 1(c)). Therefore the same test was executed again, but this time only with the first trial as a reference point. In this case there was a habituation effect from the 3rd trial onwards (all trials: $F(2,39)>3.08$, all $P_{S}<0.05$ ).

(4)

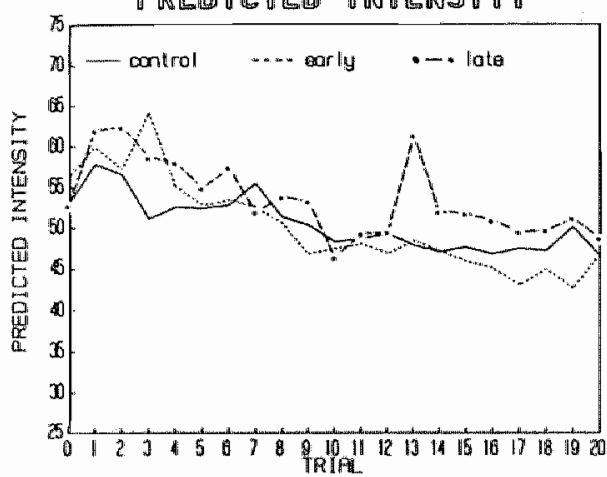

CERTA INTY PRERICTED IMTENS ITI

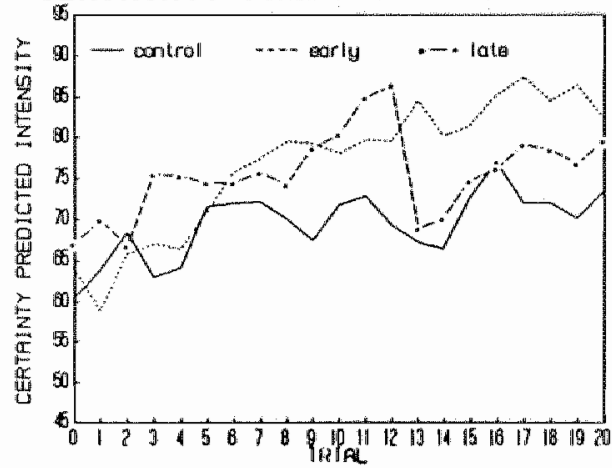

(b)

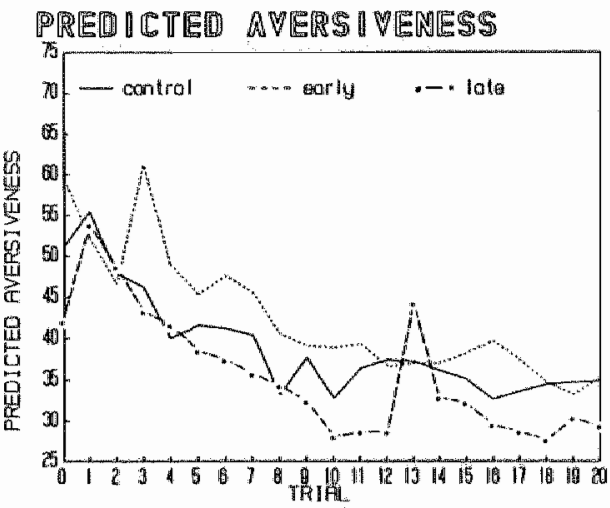

(4)

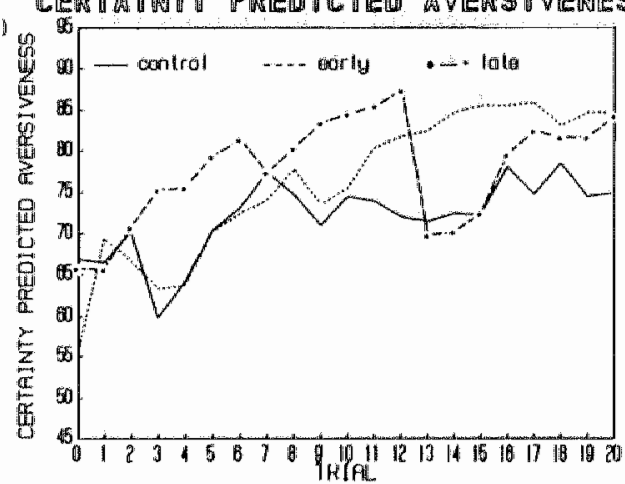

Figure 2. Mean predictions of pain intensity (figure 2(a)) and pain aversiveness (figure 2(b)), mean certainty of predicted intensity (figure $2(c)$ ) and mean certainty of predicted aversiveness (figure 2(d)) for the early, late and control conditions. 
(6) i

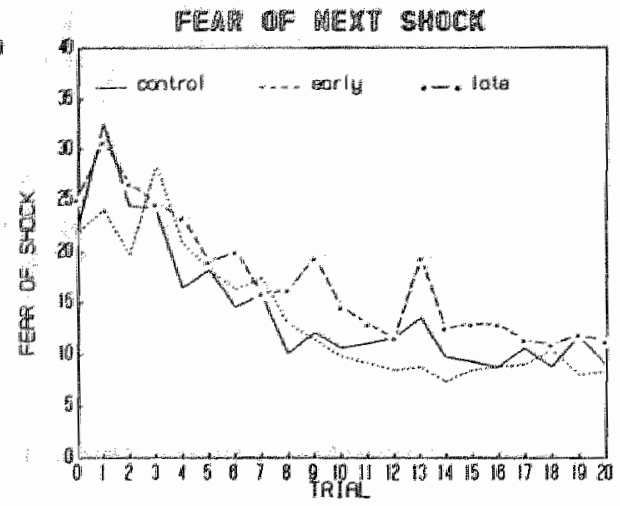

(4)

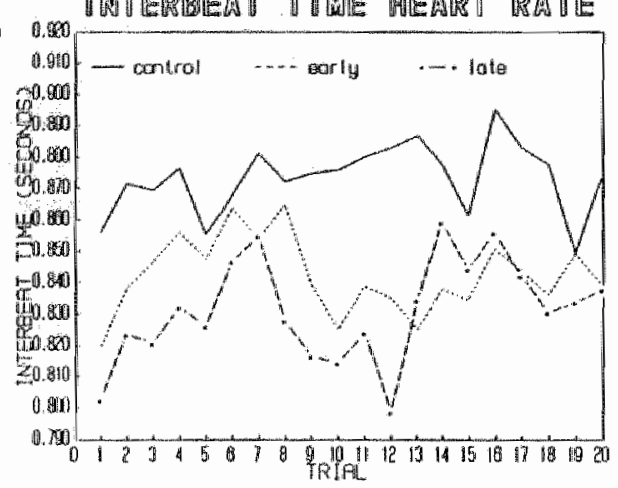

(b)

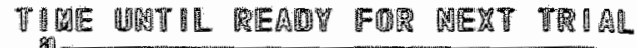

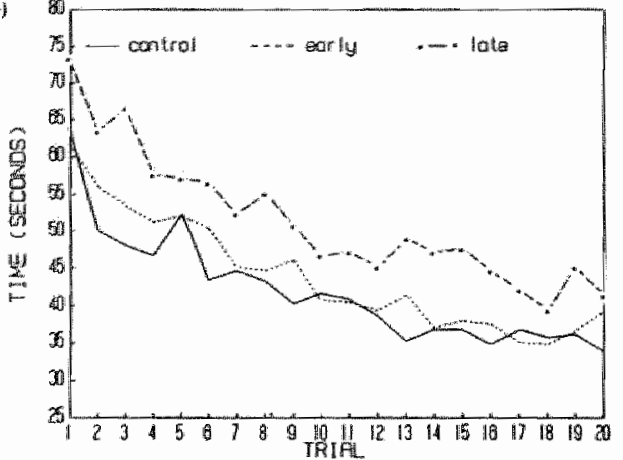

(d)

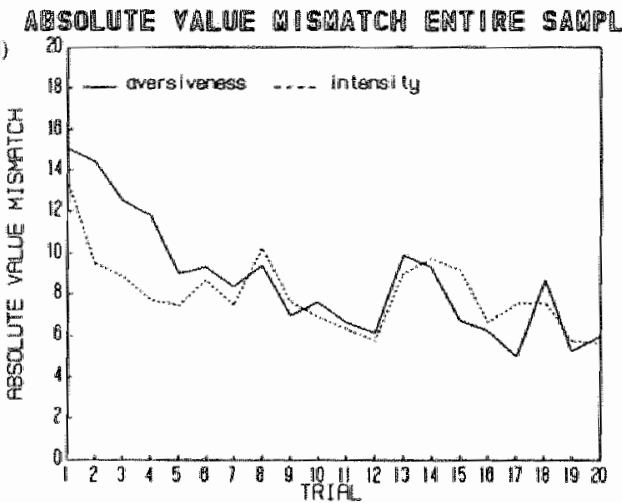

Figure 3. Mean fear of shock (figure 3(a)), time (in seconds) following shock at trial i until $S^{\text {'s }}$ indication that he is ready for the next shock (figure 3(b)), mean inter-beat intervals (heart-period) of the 12 sec prestimulus period (figure $3(\mathrm{c})$ ) and accuracy of pain predictions (defined as | prediction - experience |) (figure 3(d)).

As can be seen in figure 1(d), FPV-responses did not show habituation (all trials: $F(2,39)<2.40$, all $p s>0.06)$. There is no clear pattern of habituation in the prestimulus heartpeniods (see figure 3 (c)). The trials $4,6,7,8,14,16$ and 17 showed significantly larger heartperiods (all $F(2,39)>3.18$, all $p s<0.04$ ).

In sum, the subjective variables measured via the VAS-scales habituate. There is some evidence that the skin conductance responses habituate; the other physiological responses (FPV and HR) do not show any clear habituation.

Accuracy of predictions will increase with practice (hypothesis 6)

There is some evidence that accuracy did increase over the trials. A trend analysis was executed to investigate whether the absolute difference between predicted pain intensity/aversiveness and experienced pain intensity/aversiveness decreases over the trials. The mean absolute scores of the intensity and aversiveness mismatches are depicted in figure 3(d). There is a significant exponential decrease in the difference between prediction and experience over the trials (intensity mismatches: $F(1,41)=20.4, p<0.001$; aversiveness mismatches $F(1,41)=28.04, p<0.001$ ). Hypothesis 6 was confirmed. 
Increase of certainty over time (hypothesis 7)

From the 5 th trial onwards there was a significant increase in the certainty as to predicted pain intensity (see figure 2(c)) as well as in the certainty as to predicted aversiveness (see figure 2(d)): all trials: $F(2,39)>3.78$, all $P_{S}<0.03$.

Fear of shock will decrease over time (hypothesis 8)

From trial 4 onwards the fear scores (see figure 3 ) were significantly lower (all $F(2,39)$ $>15.35$, all $P_{s}<0.001$ ).

Table 1 . Effects of early and late underprediction

\begin{tabular}{|c|c|c|c|c|c|c|}
\hline \multirow[t]{2}{*}{ variable } & \multicolumn{3}{|c|}{ early (1) } & \multicolumn{3}{|c|}{ late 2) } \\
\hline & trial i & $t$ & $p$ & trial i & $t$ & $p$ \\
\hline \multirow[t]{3}{*}{ intensity } & 3 & 1.85 & 0.036 & 13 & 3.90 & 0.000 \\
\hline & 4 & -0.85 & 0.200 & 14 & 1.97 & 0.028 \\
\hline & 5 & -0.13 & 0.449 & 15 & 1.41 & 0.167 \\
\hline \multirow[t]{3}{*}{ aversiveness } & 3 & 2.74 & 0.005 & 13 & 4.09 & 0.000 \\
\hline & 4 & 0.60 & 0.226 & 14 & 1.67 & 0.052 \\
\hline & 5 & 0.97 & 0.168 & 15 & 1.68 & 0,051 \\
\hline \multirow{3}{*}{$\begin{array}{l}\text { predicted } \\
\text { intensity }\end{array}$} & 3 & 3.50 & 0.001 & 13 & 3.28 & 0.001 \\
\hline & 4 & 0.61 & 0.275 & 14 & 1.23 & 0.113 \\
\hline & 5 & 0.40 & 0.347 & 15 & 1.32 & 0.098 \\
\hline \multirow{3}{*}{$\begin{array}{l}\text { predicted } \\
\text { aversiveness }\end{array}$} & 3 & 4.08 & 0.000 & 13 & 4.00 & 0.000 \\
\hline & 4 & 2.87 & 0.004 & 14 & 1.93 & 0.031 \\
\hline & 5 & 1.83 & 0.037 & 15 & 1.23 & 0.114 \\
\hline \multirow{3}{*}{$\begin{array}{l}\text { certainty } \\
\text { intensity }\end{array}$} & 3 & -0.05 & 0.480 & 13 & -4.50 & 0.000 \\
\hline & 4 & -0.22 & 0.414 & 14 & -2.62 & 0.007 \\
\hline & 5 & -0.03 & 0.488 & 15 & -3.26 & 0,001 \\
\hline \multirow{3}{*}{$\begin{array}{l}\text { certainty } \\
\text { aversiveness }\end{array}$} & 3 & -0.06 & 0.476 & 13 & -3.76 & 0.001 \\
\hline & 4 & -0.35 & 0.363 & 14 & -3.45 & 0.001 \\
\hline & 5 & -0.09 & 0.464 & 15 & -3.66 & 0.001 \\
\hline \multirow[t]{3}{*}{ fear of shock } & 3 & 2.08 & 0.022 & 13 & 2.77 & 0.005 \\
\hline & 4 & 2.35 & 0.012 & 14 & 1.52 & 0.068 \\
\hline & 5 & 1.75 & 0.044 & 15 & 1.67 & 0.052 \\
\hline \multirow[t]{3}{*}{ SCR } & 3 & 0.04 & 0.486 & 13 & 3.17 & 0.002 \\
\hline & 4 & -1.64 & 0.055 & 14 & 0.30 & 0.383 \\
\hline & 5 & -0.66 & 0.258 & 15 & 0.32 & 0.374 \\
\hline \multirow[t]{3}{*}{ FPV } & 3 & 0.23 & 0.412 & 13 & 2.87 & 0.004 \\
\hline & 4 & -0.38 & 0.354 & 14 & 2.53 & 0.009 \\
\hline & 5 & -0.74 & 0.233 & 15 & 1.08 & 0.146 \\
\hline
\end{tabular}

Note: 1) Effects of the early underprediction are based on differences between trial i and trial 2 as contrasted with the combined control and late conditions; 2) Effects of the late underprediction are based on the differences between trial $i$ and trial 13 as contrasted with the combined control and early conditions. 


\section{New hypotheses}

Smaller or larger effects of late underprediction? (hypothesis $9(a)$ and (b))

There are indications that the late induced underprediction had greater consequences than the early underprediction. First, on all VAS-scales a significant effect of the late induced underprediction was found. This is not the case at the early underprediction. Second, the effects of the late underprediction are significantly larger than the effects of the early underprediction in some aspects, viz. in: experienced intensity of pain ( $t=6.80$, $p=0.015,2$-tailed), certainty of the predicted pain intensity $(t=2.53, p=0.02,2$-tailed $)$ and skin conductance $(t=2.15, p=0.04,2$-tailed $)$.

\section{Dishabituation after underpredicted pain? Mypothesis $10(a)$ and (b))}

After the early underprediction, the prolonged increases in scores of fear and of the predicted aversiveness of the shocks are remarkable (see table 1). The early underprediction did not have any long-term effects on the physiological responses.

After the late induced underprediction all VASs but one showed significant long-term effects (see table 1). Only the predicted intensity scores failed to show a significantly increase. With respect to the physiological responses, increased FPV-responses were still present at the 14th trial $(t=2.42, p=0.011)$. Concerning the late underprediction, hypothesis 10(b) was confirmed: the induction did have a dishabituation effect. Concerning the early underprediction, there were hardly any indications of dishabituation.

\section{DISCUSSION}

The present study replicates most of the findings of the study by Arntz and van den Hout (1988) on the generalizability of Rachman's match/mismatch model and gives further support to its use as a general model for human processing of experiences with aversive stimuli. The replicated findings can be summarized as follows:

1. Underpredicted pain is followed by an immediate increase in expected intensity and aversiveness of the next painful experience.

2. After an underprediction, the predictions of aversiveness are higher for more than one trial, and need several corrective experiences before they become accurate again.

3. Underpredicted pain delays the reduction of fear of the painful stimulus, and makes $S \mathrm{~s}$ uncertain about their expectations for a considerable time.

4. With practice, predictions become more accurate and certainty about these predictions increases.

5. Despite an underpredicted painful experience, most responses ultimately habituate (experienced intensity and aversiveness, fear of shock, SCR).

Some findings differed from the study by Arntz and van den Hout, however. First, in the study by Arntz and van den Hout, fear of shock did not increase after an underprediction. In the present study, fear of shock immediately increased after an underprediction. Since the $N$ in the present study is larger, there appears to be more evidence that underpredicted pain is generally followed by increased anticipation anxiety. Second, in the study by Arntz and van den Hout an (early) underprediction was followed by an immediate increase in certainty about (higher) pain prediction. In the present study certainty did not change after the early underprediction, but decreased after the late induced mismatch. The long-term effect of decreased certainty after an underprediction was found in both studies and therefore appears to be robust. The previous finding of quickening of $S$ s indications (after 
an underprediction) that they were ready for the next trial was not replicated. After the early mismatch, only a (n.s.) trend towards acceleration was found; whereas after the late underprediction, a trend opposite to deceleration was found. Thus, there does not appear to be any general effect of underpredicted pain on the time that $S$ s wait for the next trial. The last two findings on certainty and speed may indicate different effects of early ws late underpredictions in a series of aversive experiences, and will be discussed later on:

Besides the (replicated) findings that further support the applicability of Rachman's match/mismatch model to pain and aversive experiences in general, the present study indicates that underpredictions can have different effects depending on the degree of certainty that $S$ s have their expectations. The late underprediction, which occurred when there was a high degree of certainty, had a much stronger impact than an early underprediction, when $S$ s were still relatively uncertain about their expectations. It seems that the positive effects of habituation disappear immediately when the stimulus clearly exceeds what is strongly expected. Furthermore, this underprediction has dishabituating effects on the subsequent experiences with the original intensity of the stimulus: a painful shock of the original intensity is still experienced as more intense and more aversive, and causes stronger physiological responding. For some responses, this dishabituation is found during several trials. The implications of this finding are important.

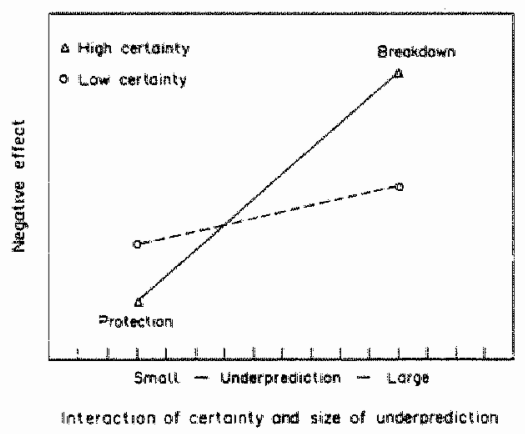

Figure 4. Illustration of the interaction between degree of underprediction and certainty of expectation. When the underprediction is small, high certainty about the expected intensity can protect the $S$ against effects of the mismatch: the effects are smaller than if the $S$ would have been certain. When the underprediction is large, the effects of an underprediction are more dramatic when the $S$ was very certain about what to expect than when the $S$ was uncertain: the underprediction causes a sort of breakdown in the schema of the $S_{\text {w }}$

Theoretically, it may clarify the relationship between matches/mismatches and the habituation process. The milder effects of the early mismatch indicate that as long as $S \mathrm{~s}$ are uncertain about their expectations (that is, they employ wide ranges in their expectations), an underpredicted aversive event is relatively less disturbing because it probably falls somewhere within the range of expectation. The finding in both studies that certainty does not decrease after an early underprediction, whereas certainty decreases immediately after a late underprediction, is in line with this analysis. When the $S$ is very certain about what to expect, small deviations from expectation (most spontaneous mismatches as analyzed in the studies by Arntz and van den Hout and in those by Rachman et al. are 
probably relatively small) do not disturb the Ss "model" of the stimulus. It seems as if new information is prevented from influencing the $S$ 's model of the stimulus. Thus, it is possible for the suppression of the effects of the stimulus (habituation) to continue and expectancy (the model) will be adjusted somewhat, but on the whole remains stable. However, large deviations from what is expected with high certainty can be very disturbing and may cause a sort of breakdown of the $S$ s model. Therefore a tentative conclusion can be drawn that high certainty (a strong model) can protect the $S$ against small deviations from what is expected. However, large deviations have more dramatic effects when the $S$ feels certain than when the $S$ feels uncertain. The different effects of underprediction as a function of certainty are illustrated in figure $4 .^{2}$

The higher impact of the painful shocks after the late underprediction may be attributed to the heightened expectations, which could bias Ss' perception. However, there are two reasons why this explanation seems improbable. First, the early underprediction also leads to a heightened expectation, but not to a greater impact of the subsequent trials. The second reason is derived from a study by Epstein and Clarke (1970). In this study $S s^{\prime}$ expectations were manipulated by means of information. Ss who overestimated the aversiveness of a loud tone initially reacted more strongly, but eventually rated the tone as less loud than correctly estimating Ss and underestimating Ss did. Epstein (1973) concludes that two processes may be operating: first, an immediate process governed by the expected impact of the stimulus - which may initially 'cause perception to function as a self-fulfilling prophecy' (p.102); and the second process: a 'reappraisal' which 'is influenced by the contrast between the expected and the real stimulus' (p.102). Whereas this hypothesized second process is able to explain the rebound effect found in the subjective experiences of the trial after the early underprediction ( $S$ s experienced it as less painful, see also Arntz and van den Hout, 1988), the experienced higher impact of the trials after the late mismatch cannot be explained by it: the reappraisal is still heightened. Therefore, the most plausible explanation is that the late underprediction had a dishabituating effect, that is, it made the active suppression of the impact of the stimulus more difficult.

The paradox of two processes involving expectancy - habituation on a more fundamental level, and the forming of conscious expectancies on a higher level of the central nervous system - that are seemingly unrelated, can be resolved. The processes probably have the same mental model of the experience in common. ${ }^{3}$ However, the point estimates of predictions that are usually asked for in match/mismatch studies, represent only one aspect of this mental model. The subjective certainty of the model and the range of what can be expected probably also play a part in the suppression process that is assumed to cause habituation. Now it becomes understandable why $S$ s tend to overestimate aversive events and why overpredictions need more corrective experiences to become accurate: overpredicted aversive events probably do not disturb the suppression of the impact of the stimulus, whereas underpredictions may have this effect. Furthermore, it can be hypothesized that especially underpredictions may have dishabituating effects (see also Rachman

${ }^{2}$ It should be noted that certainty was not experimentally manipulated, but covaried with the number of trials. Therefore, this interpretation is speculative and direct empirical tests are needed.

The first author no longer holds this view so strictly. See section 2.3 for methodological criticism and a more stringent empirical test. 
\& Lopatka, 1986b). Their significance for the organism is greater: unexpected intense pain may indicate that the stimulus could exceed intolerable limits. Pain that is unexpectedly intense can, therefore, acquire a different meaning from pain that is predictably below a tolerable limit. Since the meaning of pain can influence its subjective impact, pain that unpredictably exceeds expectations may be experienced as more intense - even when it is reduced to a lower level (see Arntz and Schmidt (1989) for a more extensive discussion of this issue).

Finally, the present findings are also clinically important. First, they indicate that after an underpredicted aversive event (like a panic attack, a stab of pain, a medical treatment, etc.) sensitizing may occur so that experiences of lower intensity can be experienced as more intense, aversive or painful than before.

Clinical problems that are characterized by varying intensity are therefore hampered by a specific obstacle; the process of habituation will frequently be disturbed and the problems will persist. In a more general sense, a strongly underpredicted aversive event, or a powerful interruption of a strongly believed schema may have disintegrating (neurotic) effects and may lead to a generalized sensitization to events that were previously experienced as less aversive.

"Section 2.3 indicates that this probably holds for fear, other anticipatory phenomena, and physiological responding to pain, but not for subjective pain experiences. 


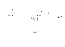




\section{SECTION 3.2. \\ THE RELATIONSHIP BETWEEN UNDERPREDICTED PAIN AND ESCAPE ${ }^{1}$}

\section{SUMMARY}

Escape and avoidance that are disproportional to the danger of the pertinent stimulus are important clinical problems that are often related to inaccurate (catastrophic) expectations. One possible source of such expectations is a prior, underestimated aversive experience. In the present experiment the hypotheses that underestimated pain leads to escape and that it leads to avoidance were tested. In order to control for the effect of the intensity of the pain stimulus, a control group that received 20 pain stimuli of high intensity was formed. Subjects in the experimental condition received 17 pain stimuli of low intensity and 3 of (unexpectedly) high intensity (experimentally induced underprediction). Underprediction of the high intensity stimulus was significantly related to escape, but not to avoidance. The results suggest that the way in which avoidance was operationalized accounts for this. The support of the hypothesized relationship between underpredicted pain and escape is an important finding, however.

\section{INTRODUCTION}

Underestimation of an aversive experience can have negative effects on its immediate and long-term impact. There is evidence that an underestimated painful stimulus is experienced as more intense and aversive than a correctly estimated stimulus (Arntz \& Lousberg, 1990). Furthermore, it has been shown that underestimation has negative longterm effects on fear of pain and on predictions of pain, which remain inaccurately high for some time (Arntz \& van den Hout, 1988; Arntz \& Lousberg, 1990) ${ }^{2}$. Rachman and coworkers developed the match/mismatch model to describe such phenomena with respect to anxiety and panic (Rachman \& Lopatka, 1986a; Rachman \& Bischard, 1988). The relationship between inaccurately predicted anxiety and behaviour related to anxiety, escape and avoidance, is central in their ideas. It appears that clinical avoidance behaviour is related to inaccurately high expectations of the aversiveness (anxiety, pain, etc.) of the avoided experience (see Rachman \& Bischard, 1988). The same is probably true of escape, as can be observed in panic patients who, during a panic attack, escape from situations in which they expect an immediate disaster. Escape and avoidance are very important clinical issues, not only because they cause large subjective problems in themselves, but also because they prevent the $S$ from testing his predictions and from adjusting them in case they are inaccurate. However, clear controlled experimental evidence that it is possible that underestimated aversive events promote escape and avoidance is still lacking. In addition to being a clinical impression, this can be expected

\footnotetext{
${ }^{1}$ Reprinted with permission from Behaviour Research and Therapy, 28, Arntz, A., van Eck, M., de Jong, P. \& van den Hout, M., The relationship between underpredicted pain and escape, 1990, Pergamon Press PLC.
}

${ }^{2}$ See, however, section 2.3 for an empirical refutation of this notion. 
theoretically, since (1) underestimated aversive events seem to be more aversive than correctly estimated events of identical intensity ${ }^{3}$, and (2) underestimation leads to prolonged overestimation, which is (probably) related to avoidance. Furthermore, it would fit in with modern cognitive reformulations of avoidance behaviour in which expectations play a central role (Seligman \& Johnston, 1973; Gray, 1975) if it were shown that underestimation may lead to escape/avoidance; and it might clarify one of the causes of escape and avoidlance.

Farlier attempts to measure escape and avoidance in match/mismatch experiments failed to produce clear results. Rachman and Lopatka (1986b) found that underpredicted fear was related to subsequent slower approach behaviour and to more hesitations during the approach. Arntz and van den Hout (1988) found that $S$ s hastened their indications that they were ready for the next trial after underpredicted pain, which can be interpreted as a tendency to escape from the experiment as a whole. However, in another study (Amtz \& Lousberg, 1990), this finding was not consistently replicated. In preparing the present study, we were impressed by the scarcity of laboratory research with human $S$ s on the spontaneous generation of escape and avoidance behaviour. Given the importance of the clinical problems of escape and avoidance, this is amazing. Most studies that were found (see Higgins \& Morris, 1984, for a review), instructed or trained Ss carefully in avoiding and therefore put a considerable experimental pressure on the $S \mathrm{~s}$ to avoid. When such procedures are used, exceptionally high rates of avoidance are found (see Higgins \& Morris, 1984). Such procedures do not appear to be relevant to the study of the spontaneous development of escape/avoidance. The procedure described below was intended to be less demanding as to escape and avoidance.

The aim of the present study is to test the hypotheses (1) that an underpredicted painful experience is more likely to lead to escape and (2) that it is more likely to be followed by avoidance behaviour than a correctly predicted painful experience of identical intensity. If underestimation has a specific effect, it should be observable separately from the effects of the intensity of the painful stimulus. In order to test these hypotheses, two groups were formed. Ss in the control group received 20 painful stimuli of high intensity. Ss in the experimental group received an equal number of stimuli, 17 of low intensity (just above pain threshold) and 3 (unexpected) of the level of intensity of the control group. This procedure reliably induces underestimations (Arntz \& van den Hout, 1988; Arntz \& Lousberg, 1990). Escape from a pain stimulus was possible by pressing a button. It was possible for $S s$ to avoid pain stimuli by reducing the number of stimuli in the experiment.

It was expected that $S \mathrm{~s}$ in the experimental group would escape from and avoid pain of high intensity more often than $S \mathrm{~s}$ in the control group.

\section{METHOD}

\section{Subjects}

Subjects were 26 students of Health Sciences who participated voluntarily and received a small remuneration. There were 22 women and 4 men. Mean age was $20 \mathrm{yr}$.

\footnotetext{
${ }^{3}$ See footnote 2.
}

${ }^{4}$ Physiological and subjective data were also obtained. They are discussed in section 3.4 . 


\section{Materials}

Materials used were a Dutch version of the Eysenck Personality Questionnaire (EPQ, Sanderman, Eysenck \& Arrindell, 1988), and visual analogue scales (VAS) measuring predicted pain, experienced pain and fear of the next pain stimulus ${ }^{2}$.

\section{Apparatus}

Painful stimulation was given via electrodes attached to the $S$ 's right ankle. The stimulation increased towards its maximum during $2 \mathrm{sec}$, remained at that level for $6 \mathrm{sec}$, and gradually decreased during 2 sec. The electrical pain stimulation was produced by a Siemens Neuroton 627 approved of for use with patients. Three seconds before the pain stimulus, a slide projector projected a white rectangle on the wall as a warning signal for $1 \mathrm{sec}$. Ss could press an escape button connected with a device for measuring reaction time to terminate the stimulation. Reactions within the stimulation duration of $10 \mathrm{sec}$ were classified as escapes. Avoidance was measured by means of a device with five buttons (button 1: reduction by one trial, button 2: reduction by 2 trials, etc.) connected with five bulbs in the control-room. A microcomputer (PDP Minc-11) controlled the experiment.

\section{Procedure}

Before entering the experiment room, Ss filled out the EPQ. On the basis of the Neuroticism score and sex, matched pairs were formed. The first $S$ of a pair was assigned to the experimental condition, the second to the control group. After $S$ s had entered the experiment room and electrodes had been fastened (for heart rate, skin conductance, finger and ear plethysmogram, and pain stimulation) ${ }^{2}$, Ss were told that they were participating in a psychophysiological study on pain. The shock level was determined in steps of $0.4 \mathrm{~mA}$. In the experimental condition, the first painful intensity $>50 \mathrm{~mm}$ on the VAS measuring pain was chosen (mean subjective level $54 \mathrm{~mm}$, mean intensity $2.6 \mathrm{~mA}$ ). For a $S$ in the control group the intensity was further raised until the subjective pain was somewhat higher on the VAS than the subjective pain score of the matched experimental $S$ at the heightened trials (mean subjective level $65 \mathrm{~mm}$, mean intensity $3.4 \mathrm{~mA}$ ). The subjective level was significantly higher in the control group than in the experimental group $(t(24)=4.02, p=0.001)$, as was the objective level $(t(24)=2.01, p=0.03$, 1 -tailed). Ss were instructed that they would receive an (unspecified) number of pain stimuli, and that each stimulus would be preceded by the warning signal. Furthermore, the Ss were told that, although the objective intensity would remain constant, subjectively experienced pain could fluctuate; that they could terminate the stimulus by pressing the stop-button; and that they could reduce the number of stimuli by pressing one of the five buttons (described above) after each trial. Ss were instructed to complete the 3 VASs after each trial. Ss in the control group received 20 shocks of the (high) level determined at the start of the experiment. $S$ s in the experimental group received 17 shocks of the (low) level that was determined at the start, and 3 heightened shocks: at the 7,12 and 17th trial the objective level (in $\mathrm{mA}$ ) was heightened by $33 \%$. The objective intensity of the high intensity pain stimulus was the same for the two groups $(t(24)=0.26, p=0.80)$. 


\section{RESULTS}

\section{Experimentally induced underpredictions}

The experimental $S s$ underpredicted the experienced pain at the 7th (discrepancy $=8$ $\left.\mathrm{mm}, t(12)=4.35, p<10^{-3}\right)$, at the 12 th $\left(12 \mathrm{~mm}, t=4.59, p<10^{-3}\right)$ and at the 17 th trial $(8$ $\left.\mathrm{mm}, t=4.03, p=10^{-3}\right)$. Thus, the experimental manipulation succeeded in inducing underpredictions. Figure 1 and 2 show experienced and predicted pain of both groups on a trial by trial basis.

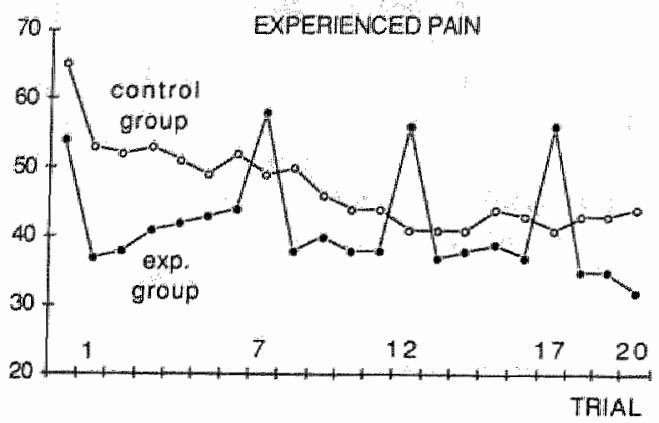

Figure I. Experienced pain of control and experimental group during the series of painful shocks. In the experimental group the stimulus intensity was increased at the 7, 12 and 17 th trial. $S \mathrm{~s}$ in the control group received stimuli of a high intenisity matched to the intensity of the experimentally increased stimuli in the experimental group. Trial 0 denotes the pretrial measurement where shock level was assessed.

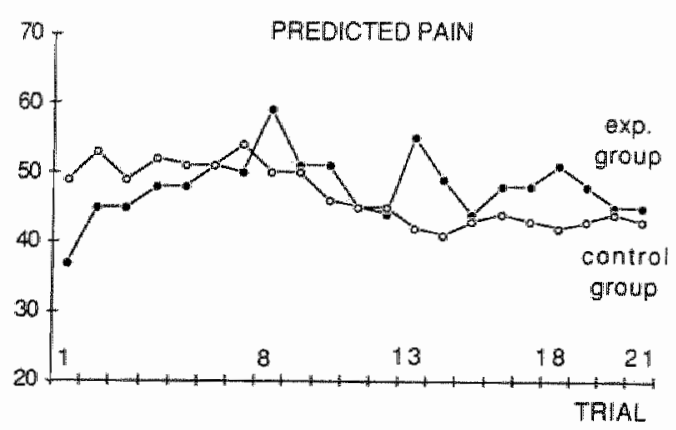

Figure 2. Predictions of pain intensity in both groups. As can be seen, the experimentally increased stimuli in the experimental group were underpredicted and caused increased pain predictions.

\section{Escape}

There was one $S$ in the control group who terminated the pain stimulus once. Inspection of the subjective data showed that the $S$ had underpredicted the 16th trial (predicted level 35, experienced level 46). Thus, the frequency of escape from a high intensity stimulus was $1 / 260(0.4 \%)$ in the control group. In the experimental group, there were four $S s$ who terminated the stimulus at least once (two $S$ s made one escape, one made three escapes, and one made four escapes). Eight of the 9 escapes were from the experimentally heightened stimulus. When analyzed over all 20 trials, the number of $S \mathrm{~s}$ escaping was higher in the experimental condition, as hypothesized, but was statistically only marginally significant (Fisher's exact test: $p=0.08$, 1-tailed). The frequency of escape from a strong stimulus is, of course, a more pertinent measurement. This frequency was much higher in the experimental condition ( 8 out of $39 ; 20.5 \%$ ) than in the control group (0.4\%). The difference was highly significant (Fisher's exact test: $p=3.5 \mathrm{x}$ $\left.10^{-7}\right)$. A $t$-test with the individual frequency of escape as dependent variable also revealed a significant between group effect $(t(12)=1.94, p<0.05,1$-tailed, separate variance estimates). It might be argued that the frequency of escape from a high intensity stimulus 
gradually decreased in the control group because of habituation to the stimulus. To control for this habituation effect, the number of escapes from a high intensity stimulus in the experimental group (8 out of 39) was contrasted with the number of escapes from the first stimulus (before habituation) in the control group (0 out of 13). The difference was significant (Fisher's exact test: $p=0.04,1$-tailed) as was the difference between the groups as to the frequency of escape $(t(12)=1.98, p<0.05,1$-tailed, separate variance estimates).

\section{Avoidance}

In each condition there were only two $S s$ who made avoidance responses. In the control condition one $S$ skipped several trials, "because the stimulus was not painful anymore (as she stated afterwards), and for a study on pain there would, therefore, be no point in completing the full program." Inspection of the subjective data indeed showed strong habituation of this $S$. The other $S$ of the control group made avoidance responses after a strongly (spontaneously) underpredicted stimulus. There were no significant differences between the conditions with respect to avoidance responses.

\section{DISCUSSION}

The findings of the present study support the general hypothesis that an underestimated painful experience is experienced as more aversive than an accurately estimated painful experience of identical intensity. It is more likely that an underestimated painful experience will lead to escape than that a correctly estimated experience will do so. The present findings are even more significant because the level of intensity of the stimulus was controlled for: the organism seems to be able to tolerate strong pain better when it is correctly expected than when it is unexpectedly strong. The results suggest that it is not so much the level of pain or aversiveness that matters, but rather the unpredictability.

However, was it really the underestimation, and not the intensity, that mattered? Although the control $S s$ received pain stimuli of a somewhat higher subjective intensity at the start than that of the experimentally increased stimuli in the experimental group, control $S s$ on average experienced less subjective pain during the series than experimental Ss did at the intensified trials, because of habituation in the control group and the dishabituating effect of underestimated pain in the experimental group. Thus, theoretically it could be reasoned that it was the higher experienced pain in itself that was responsible for the high escape rates. However, at the first trial(s) - before habituation could have taken place - the control $S$ s did not escape at all. The authors therefore feel confident that the underprediction in itself promoted escape.

Possible mechanisms underlying higher likelihood of escape from underpredicted pain may be: (1) the dishabituating effect of an underpredicted pain stimulus, making the inhibition of the effects of the stimulus ineffective (see Arntz \& Lousberg, 1990, for experimental evidence), an experience which may make the stimulus more aversives; and (2) the different meaning the stimulus gets when it is unexpectedly intense - that of a stimulus that can become intolerable at any moment. It is well known that the meaning and significance of pain can influence the way in which it is experienced (see Arntz \& Schmidt, 1989, for an extensive discussion of this issue). Be that as it may, the present

\footnotetext{
${ }^{5}$ See footnote 2.
} 
study validates clinical observations on escape and is theoretically relevant to Rachman's match/mismatch model in giving strong evidence of a causal connection between inaccurately estimated aversive events and escape behaviour.

Whereas the effects of underpredicted pain on escape are clear, the present study failed to show that underpredicted pain is followed by avoidance behaviour. It should be noted that only two $S s$ in each condition made any avoidance response at all. Therefore, there was hardly any variance for showing possible differences. We assume that the way in which avoidance behaviour was operationalized accounts for this. First, it may not have been salient to the $S$ that, and how, the number of trials was reduced if he pressed a button. Second, there was no direct connection between avoidance response and a specific trial. Third, $S \mathrm{~s}$ may have believed that they should not reduce the number of trials (which is supported by the small number of $S \mathrm{~s}$ who made avoidance responses) and be brave. The attempt not to put too much pressure on the $S$ to avoid may have been too successful. Thus, by a different operationalization and less experimental demand on tolerance of pain, the link between underpredicted pain and avoidance behaviour may be found. Such an attempt will now be undertaken. 


\section{SECTION 3.3. AVOIDANCE OF PAIN OF UNPREDICTABLE INTENSITY}

\section{SUMMARY}

Unpredictability of the intensity of an aversive event might be an important factor in producing negative effects of the event, especially if the UCS becomes stronger than could have been expected. The present experiment tested the hypothesis that unpredictability of intensity of a painful stimulus contributes to avoidance behaviour. The experiment was concealed in a shock working-up procedure, which was done to assess the pain levell $S$ s were willing to tolerate in a subsequent experiment. The experimental $S$ s, who received an unannounced sudden increase of the pain stimulus during the working-up procedure, tolerated less pain on a subjective as well as on an objective level (avoidance of high levels of pain) than the control $S \mathrm{~s}$, who received the stimuli in a predictable pattern. The results support the hypothesis that unpredictability of intensity of an aversive event contributes to avoidance behaviour.

\section{INTRODUCTION}

There is growing evidence that besides unpredictability of the occurrence of aversive events, unpredictability of the intensity of aversive events produces various disturbances to the organism, such as fear of the event that exceeds the fear of a predictable aversive event. In various experiments it has been found that pain that is unexpectedly stronger leads to immediate increases in predictions of pain expected at later trials, to a slow reduction of these predictions despite disconfirmatory experiences, to long-lasting fear of the pain stimulus, and to escape from the underpredicted event (Arntz \& Van den Hout, 1988; Arntz \& Lousberg, 1990; Arntz, Van Eck, De Jong \& Van den Hout, 1990; Arntz, Van Eck \& De Jong, 1991). Theoretically, it could be hypothesized that unpredictability of the intensity of aversive events is also related to avoidance behaviour, especially if the intensity is larger than could be expected. However, to the best of the present authors' knowledge, no study has been published that clearly shows that unpredictability of intensity of an aversive UCS is related to human avoidance. In an unpublished study (Rachman, 1990), it was found that an unexpected sudden decrease of the distance between a phobic $S$ and the feared object resulted in subsequent avoidance. Indirect evidence has been reported in a study by Rachman and Lopatka (1986): underpredicted fear was related to subsequent slower approach behaviour and to more hesitations during the approach of the feared object. However, in this study the underprediction of fear was not experimentally manipulated, which leaves the issue of causality open.

From a clinical perspective it might be interesting if it could be demonstrated that unpredictable intensities of aversive events are related to avoidance behaviour, because there seem to be severall forms of avoidance behaviour that are based on unpredictability of intensity rather than of occurrence. Especially unpredictability of the upper limits of the aversiveness of the event may be important, as in dental phobia or panic attacks, where

\footnotetext{
'Reprinted with permission from Behaviour Research and Therapy, 29, Arntz, A., van Eck, M. \& de Jong, $P_{.}$, Avoidance of pain of unpredictable intensity 1991 , Pergamon Press PLC.
} 
patients do not seem to fear moderate intensities of the aversive event, but rather sudden, unpredictable increases to intolerable levels. Indirect evidence for this proposition stems from the relationships that have been found between avoidance of dental treatment and (catastrophic) predictions of pain expected during treatment (cf. Wardle, 1983; Lindsay, 1983), and between agoraphobic avoidance and expectations of panic (Craske, Rapee \& Barlow, 1988) or subjective probability of catastrophic events (Warren, Zgourides \& Jones, 1989). From a theoretical perspective, it seems that unexpected increases in the intensity of an aversive stimulus yield important information for the organism. If an aversive stimulus increases unexpectedly in intensity, the $S$ cannot be certain any more whether the intensity will remain below bearable limits. In other words, unexpected increases of the intensity might lead to distrust of the stimulus, and consequently to avoidance.

In a previous attempt to test the hypothesis that unexpected increases of a painful stimulus to a high intensity level lead to more avoidance behaviour than predictable experiences with identically high intensities of the stimulus, Amtz et al. (1990b) found evidence for a relationship with escape behaviour, but did not observe more avoidance behaviour. In both conditions there was a very small number of instances of avoidance (measured by letting the $S$ s indicate whether they wanted to skip a number of trials of the series of painful stimuli). Thus, the variance was too low to allow for detection of between-group differences. Arntz et al. hypothesized that the very small number of avoidance behaviours could be explained by the considerable experimental demand put on the $S$ s to complete the series as planned or by the unclear effects of the avoidance response. Another reason might be that the possibility to escape from the stimulus might have reduced avoidance. There is evidence that if stimuli are escapable, less avoidance is observed (Bixenstein \& Barber, 1964; Higgins \& Morris, 1984). This can be understood by considering the possibility to escape as a form of controllability. The possibility of escaping increases the certainty that the stimulus can be endured without becoming intolerable.

In further pillot studies we gave $S$ s no possibility of escaping from the painful stimulation, and we tried to lessen the experimental demand to endure the stimulus. If, however, the experimental demand was lowered, very high rates of avoidance behaviour were observed, again leading to a variance that was too low. We concluded that human avoidance behaviour of new stimuli in the laboratory is easily determined by experimental demands, which makes it very difficult to study spontaneous avoidance behaviour (see for the strong effects of experimental instructions on human avoidance for instance: Matthews et al., 1977; Shimoff et al., 1981; Higgins \& Morris, 1984).

We therefore decided to hide the experimental manipulation in a phase of pain experiments in which $S$ s seem to display relatively more spontaneous behaviour: the introductory phase, in which the individual pain level is selected. In short, $S$ s participated in an experiment that started after the selection of the pain stimulus. A shock working-up procedure was employed to determine the highest level the $S$ was willing to tolerate during the experiment that would follow. In the experimental condition, an unexpectedly increased pain stimulation was given during the working-up procedure. In the control group, the working-up procedure followed a predictable pattern. A double-blind design was employed: the experimenter who welcomed the $S$ to the laboratory and communicated to the $S$ during the working-up procedure did not know to which condition the $S$ was allocated. The second experimenter, who controlled the pain stimulation in an adjacent room, randomly allocated the $S$ to one of the conditions. It was hypothesized that $S$ s who had experienced an unexpectedly increased stimulation would tolerate less pain (that is, would avoid high levels of pain) than $S$ s who had experienced predictable intensities of the 
pain stimulus. The hypothesis was tested both on a subjective level (experienced pain) and on an objective level (intensity of the painful electrical stimulation in $\mathrm{mA}$ ).

\section{METHOD}

\section{Subjects}

Subjects were 50 female students, who participated voluntary. A small remuneration was given for participating. There were $43 \mathrm{Ss}$ in the final sample (see procedure for $S$ sellection). Mean age was 20 years $(S D=1.5)$.

\section{Apparatus}

Apparatus was placed in an adjacent room. The pain stimulation was produced by means of a Siemens Neuroton 627, passed for use with patients. The electrical stimulation was delivered at the right ankle of the $S$ via two Beckman $8 \mathrm{~mm}$. Ag-AgCI electrodes, filled with Hewlett-Packard Redux Creme. Each stimulation consisted of a gradual rise during $2 \mathrm{sec}$. to the preselected level, a constant stimulation of $1 \mathrm{sec}$, and a gradual decrease during 2 sec. Skin conductance electrodes, two Beckman $8 \mathrm{~mm}$. Ag-AgCl electrodes, were attached to the medial phalanges of the second and third fingers of the nondominant hand. Since no physiological recording was done during the shock working-up procedure, in which the present experiment took place, no further details about physiological recording will be given. An intercom was used for communication between the experimental room and the adjacent control room.

\section{Materials}

Subjects rated experienced pain on $100 \mathrm{~mm}$ visual analogue scales (VAS). At the left end of the scale the indication " 0 , no pain at all" was printed, at the right end "100, extremely painful". The distance from zero to the vertical mark of the $S$ was measured in $\mathrm{mm}$.

\section{Procedure}

The present experiment was carried out during a shock working-up procedure of two other experiments. Therefore, $S \mathrm{~s}$ were first given the rationale of the experiment that was to take place after the shock working-up procedure. After giving this rationale electrodes were fastened and the shock working-up procedure was started. The $S$ was told that the purpose of this procedure was to determine the highest level of stimulation she was willing to tolerate during the experiment that would follow. It was also explained to the $S$ that in order to help her to judge the stimulation, three stimulations of each objective level would be given, and that she should indicate after each series of 3 stimulations whether she would like to try a higher intensity, keep the intensity on this level, or prefer a lower intensity. If the $S$ chose a higher or a lower intensity, a new series of 3 stimulations was given. The working-up procedure started at an objective level of $0.0 \mathrm{~mA}$. The level was increased in steps of $0.2 \mathrm{~mA}$ until tolerance level was reached. Pain-threshold was also measured, on subjective level (VAS filled in after the first series experienced as painful) and on objective level (in $\mathrm{mA}$ ). Each series of 3 stimulations was rated on the VAS after pain threshold had been reached. 
The experimental manipulation was accomplished by unexpectedly increasing the objective level of the third stimulation by $33 \%$ in the series following after the first stimulation level that was rated $>50$ on the VAS. A double-blind procedure was employed. One experimenter was seated beside the $S$, giving the $S$ the VASs and asking the $S$ whether the level should be increased. If the $S$ refused an increase, the experimenter asked the $S$ whether the level could be kept on the last intensity, or should be decreased. In case of an increase, this experimenter said "yes" via the intercom. However, in case of the furst level that was rated $>50$, the experimenter said "okay" which was the code to the second experimenter that the level of 50 was reached.

Only the second experimenter knew whether the $S$ was allocated to the experimental or to the control condition (allocation was random). The second experimenter controlled the pain stimulator. In case of an experimental $S$, the third stimulation of the first series after the code "okay" was increased. In case of a control $S$, the procedure of increasing the level after a series of 3 stimulations of the same intensity was continued. After the experimental manipulation, the normal procedure was continued until tolerance level was reached. There were $7 \mathrm{Ss}$ who had tollerance levels below 50 on the VAS. Since the experimental manipulation would be impossible, these $S s$ were deleted.

\section{RESULTS}

A MANOVA on the objective level and the subjectively experienced level at tolerance with pain threshold levels (objective and subjective) as covariates revealed a significant experimental effect $\left(F_{\text {Hoi }}(2,38)=4.35, p=0.02\right)$. Means and s.d. are presented in table 1 .

In case of subjective pain, the covariates were n.s. and were therefore deleted in the univariate test. In case of the objective level, only objective threshold level was found to be significant as covariate.

Univariate one-tailed $t$-tests (planned contrasts) revealed that $S_{s}$ in the experimental condition tolerated significantly less pain on the subjective level $(t(41)=-2.99 p<0.005$, table 1) as well as on the objective level $(t(40)=-1.78, p<0.05$, table 1). The weaker effect on the objective level should be attributed to the large within-group variances: $S$ s differed enormously in tolerance level, and therefore the between-group effect is relatively weak. In case of the subjective pain, within-group variances are relatively lower.

To summarize, the objective as well as the subjective findings clearly support the hypothesis that $S$ s tolerate less pain after an unpredicted sudden increase in pain.

Table 1. Objective and subjective pain levels

\begin{tabular}{|c|c|c|c|c|}
\hline & $\begin{array}{l}\text { Threshold } \\
\text { mean }\end{array}$ & $S D$ & $\begin{array}{l}\text { Tolerance } \\
\text { mean }\end{array}$ & $S D$ \\
\hline \multicolumn{5}{|l|}{ objective laval (mA) } \\
\hline control & 1.37 & 0.71 & 4.17 & 1.70 \\
\hline experimental & 1.27 & 0.81 & 2.78 & 3.22 \\
\hline \multicolumn{5}{|c|}{ subjective level $(0-100)$} \\
\hline control & 24.7 & 17.3 & 86.7 & 11.1 \\
\hline experimental & 36.3 & 17.9 & 74.0 & 15.5 \\
\hline
\end{tabular}




\section{DISCUSSION}

Interspersing an experimental manipulation in a shock working-up procedure appeared to be an adequate procedure to study avoidance without the common problem of experimental demand. The findings obtained with this paradigm support the hypothesis that $S$ s are willing to tolerate more pain if the intensity of the pain stimulus is predictable. If the pain stimulus becomes unexpectedly stronger, $S$ s tend to avoid high levels of the stinulus.

The lower tolerance level after unexpectedly increased pain can be explained in various ways. First, the unexpected increase may have induced a state of anxiety in the $S$ s, leading to avoidance of higher levels or even escape from the level that was experienced last (by having the stimulation level decreased). There is indeed evidence that unexpected increases in pain lead to increases in subjective anxiety (Arntz \& Lousberg, 1990; Arntz et al., 1991c), to less reduction of fear of the stimulus (Arntz \& Van den Hout, 1988), to increased physiological anticipation of the stimulus (Arntz et al., 1991c), and to escape from the stimulus (Arntz et al., 1990b).

Second, a cognitive explanation might be that the stimulus representation (see Davey, 1989, for the importance of the UCS representation in acquired fears) is qualitatively or quantitatively different after an unexpected increase compared to after correctly expected experiences. A quantitative difference might be that an unexpectedly strong stimulus is experienced as more painful, leading to a representation of the stimulus as being more painful. The higher painful quality of the stimulus might have led to avoidance. Although there are indications that an unexpectedly stronger stimulus might be experienced as more painful than a correctly expected stimulus of equal objective intensity (Arntz et al., $1990 \mathrm{~b}$ ), the present findings seem to contradict the explanation that the experimental $S \mathrm{~s}$ tolerated less objective pain because their stimulus representations were relativelly more painful. For if this explanation would be true, approximately equal, or even higher, subjective tolerance levels combined with lower objective tolerance levels would have been observed in the experimental condition, compared to the control group. The present findings indicate, however, that the experimental $S s$ experienced the tolerance levels as relatively less painful than the control Ss. A more simple explanation therefore seems to be that $S$ s did not dare tolerate higher levels of the stimulus because the stimulus acquired a different meaning for the $S$ on another dimension: as being dangerous because it suddenly can become stronger, as being untrustworthy because of being unpredictable with respect to its upper limits. Although this interpretation cannot be directly proven by the present experiment, it seems at least plausible. Moreover, it hooks on to theoretical explanations of the positive effects of controllability and predictability of aversive events, which stress the importance of the certainty that the $S$ gets, when the stimulus is controllable or predictable, that the stimulus will remain below unbearable levels (Miller, 1979). Lastly, such an explanation might also be in line with cognitive explanations of avoidance behaviour, which do not rely on (conditioned) fear as a necessary incentive for avoidance (Seligman \& Johnston, 1973).

Clinically, unpredictability of intensity seems to be important with respect to various aversive events. For instance, many panic patients seem to avoid agoraphobic situations because they expect that a panic attack, once above a certain threshold, can become increasingly stronger and leads to unbearable effects, like becoming crazy, loosing control, or dying (Clark, 1983). Similarly, some chronic pain patients might avoid activities because they believe that the pain may become intolerable (Lethem et al., 1983; Slade $e t$ al., 1983). Experiences with unpredicted increases of pain may have lead to such expectations. The same mechanism might play a part in acute pain, of which patients 
might become afraid, or which might lead patients to avoid painful treatment or to ask for medication, because of having once experienced an unexpectedly strong pain experience. Uncertainty about the upper limits of pain due to treatment seems to play an important part in dental phobics (Lindsay, 1983).

In sum, the present study supports the hypothesis that unexpected increases in the intensity of an UCS might lead to avoidance. Further studies are needed to unravel underlying mechanisms. For instance, it might be interesting to investigate the effects of a signalled increase in UCS intensity compared to the effects of an unsignaled increase as was employed in the present study. If signaling does not result in higher tolerance, the effects must be attributed the sudden sharp increase in pain intensity per se, and (habituation) processes at lower levels of the nervous system probably play a major role (note that the increase might still be formally unpredictable). If information can reduce the negative effects of such a sudden increase, expectations formed at higher levels of the nervous system play an important role. Similarly, the effects of perceived control over the large increases in pain intensity might be of interest in this respect. 


\section{SECTION 3.4. \\ UNPREDICTABLE SUDDEN INCREASES \\ IN INTENSITY OF PAIN AND ACQUIRED FEAR ${ }^{1}$}

\section{SUMMARY}

It is well-known that unpredictable accurrences of aversive events can produce various disturbances in the organism. Less is known about possible other relevant aspects of predictability. In the present experiment, the hypothesis was tested that sudden (unpredictable) increases in the intensity of a painful UCS contribute to the development and maintenance of fear of the UCS. In the experimental condition, $S$ s received 17 painful electrical stimulations of medium intensity, alternated with three strong stimulations. In the control group, $S$ s received 20 strong stimulations, which followed a predictable pattern because they were of constant intensity. Subjective fear ratings and autonomic responses (SCR, HRR) to a warning signal that preceded the UCS were higher in the experimental condition than in the control group, despite the fact that the control group received more stimuli of the high intensity leve $\rrbracket$. Before experimental manipulation HR deceleration after the warning stimulus was observed in both groups. The experimental manipulation resulted in HR acceleration after the warning stimulus, while the control $S$ s continued to show a deceleration. These findings support the notion that unpredictability of UCS intensity is important for acquired fears.

\section{INTRODUCTION}

The notion that unpredictable aversive events lead to stronger neurotic disturbances than predictable aversive events is well-known (Seligman, 1968; Seligman et al., 1971; Mineka \& Kihlstrom, 1978; Mineka \& Hendersen, 1985), although there is considerable dispute as to whether unpredictable aversive events are experienced as more aversive (cf. Furedy, 1975). Most of the research on unpredictability has been restricted to unpredictability of the occurrence of the aversive event. Less is known about the possible relevance of other dimensions (see Imada \& Nageishi, 1982, however). One exception is a study by D'Amato \& Safarjan (1979), in which rats preferred a condition in which signals preced ing the shock could be used for predicting the duration of the shock. Some research has been done on the effects of unpredictability of the intensity of aversive events. In an experiment by Freeman \& Badia (1975) it was found that rats did not prefer a predictable shock-intensity condition to an unpredictable condition. However, as the authors pointed out, shock intensity alternated according to a schedule that may have given sufficient information regarding shock intensity. Moreover, the relative importance of the intensity dimension probably depends on the range of intensity that is employed, because the larger the range, the higher the uncertainty is. This was demonstrated in a study by Marlin et al. (1979), in which it was found that rats only preferred a condition with information about shock intensity when the difference between intensities was relatively large. Similarly, in a study by Jones et al. (1966) it was found that human Ss made more information eliciting responses concerning shock intensity under conditions of high uncertainty about shock

'Arntz, A., van Eck, M. \& de Jong, P. Journal of Psychophysiology, accepted for publication. 
intensity than under conditions of low uncertainty. Thus, these experiments suggest that the higher the uncertanty about the UCS intensity is, the more preference for information about it is observed.

Clearly negative consequences of unpredictable intensity of painful shocks applied to human $S$ s were obserwed by Dimitrijevic et al. (1972): $S$ s who received stimuli of stochastic intensity did not show any habituation at all, in contrast with $S$ s who received stimuli of constant intensity.

Not only immediate reactions to the aversive stimulus might be influenced by sudden changes in intensity; other reactions appear to be influenced as well. Subjective anxiety and uncertainty about the stimulus that is to be expected are increased for a prolonged time after an unexpectedly stronger stimulus (Amtz \& van den Hout, 1988; Amtz \& Lousberg, 1990). When \$s receive an unannounced strong pain stimulus in a shock working-up procedure, their tolerance level is lower than when the working-up procedure follows a predictable course (Arntz et al., 1991b). It has been reported that unexpected decreases in intensity lead to a reduction of anxiety; an effect opposite to that caused by unexpected increases (Arntz \& van den Hout, 1988; Arntz et al., 1990a).

The abowe shows that there are indications that unpredictability of intensity of an UCS is important with respect to the impact of the aversive event and the development of anxiety. Unpredictable increases in intensity seem to be most important in this respect. Studying the effects of UCS unpredictability of intensity is not without clinical relevance: many pain problems, both acute and chronic, are not characterized by unpredictable occurrence of pain, but by unpredictable sudden increases in the intensity (e.g. pain during dental treatment, or chronic pain problems). Similarly, phobias seem to be characterized by (perceived) unpredictability of the intensity of the aversive event (panic attack, experienced anxiety, feared consequences of a situation) than by unpredictable occurrences. It should be noted that phobias are by definition characterized by a high (perceived) predictability of occurrence: the phobic stimulus is perceived as signal of occurrence. Nevertheless, (perceived) unpredictability (and uncontrollability) seems to play an important role in phobias Mineka, 1985; Barlow et al., 1985; Merckelbach, van den Hout \& van der Molen, 1987). In the laboratory, conditioning of autonomic responses by means of multiple pairings of a $C S$ to an (predictable) aversive UCS is often used as an analogue of clinical phobias. The validity of this laboratory model can be questioned on several grounds. Firstly, the usual laboratory situation, characterized by high predictability of the UCS (occurrence, intensity, duration), seems incomparable with the high levels of unpredictability perceived by phobic patients (see Mineka, 1985, for a more extensive discussion). Secondly, the responses after the CS are not always clear indices of fear: for instance, heart rate deceleration after a CS is sometimes found (e.g. Obrist et al., 1965; Somsen et al., 1983; Valins, 1966; even in animals, e.g., Di Cara et al., 1970), whereas heart rate acceleration is characteristic of most clinical fears. Thirdly, one-trial conditioning (in most cases the "trauma" seems to have been a panic attack, which is difficult to distinguish from the later anxiety and panic complaints; Ôst, 1989; Merckelbach et al., 1989), or even the absence of conditioning-like experiences seems to be common in phobias (Rachman, 1977). Unpredictable intensity of the UCS might lead to a more valid laboratory model of phobias and neurotic problems. All in all, the study of the effects of unpredictable increases in intensity of an aversive event seems to be worthwhile both for theoretical and clinical reasons.

In a $S_{1}-S_{2}$ paradigm, different components in the anticipatory $H R$ changes can be distinguished. With longer anticipatory periods $(>5 \mathrm{sec})$, there are generally three components: the first and third decelerative and the second accelerative (Bohlin \& Kjell- 
berg, 1979; Velden \& Sihnmacher, 1979; Somsen ei al., 1983). With shorter periods, the first and second, or only the first, components remain (see Bohlin \& Kjellberg, 1979 for a review). With an unavoidable aversive UCS, like electric shock, a global anticipatory deceleration is often found to dominate the three components (Headrich \& Graham, 1968; Obrist et al., 1969; Somsen et al., 1983). But sometimes a clear accelerative trend in anticipation of an aversive UCS is found, for instance during the inital phase of the series while later trials show less accelatory HR during anticipation (Velden \& Schumacher, 1979). Phobics also do not anticipate with a global anticipatory HR deceleration phobic stimuli: Hare and Blewings (1975) found that phobics showed a clear accelerative component during anticipation of a phobic stimulus, in contrast to their anticipation of nonphobic stimuli and the anticipatory HR of normals. Thus, there might be a curvilinear relationship between the anticipatory HR changes and the aversiveness of the UCS. Nonaversive (insignificant) UCSs generally lead to small decelerative and relatively larger accelerative components (Somsen $e t$ al.), mildly (predictable?) aversive stimuli to stronger decelerative components, and very aversive (phobic, unhabituated, unpredictable?) UCS, generally lead to strong accelerative components (Bohlin \& Kjellberg, 1979). The decelerative components are generally assumed to be related to the orienting response, the intake of information or outwardly directed attention, whereas the accelerative components are generally assumed to be related to a defensive reaction, the rejection of incoming information or flight/fight reactions (Bohlin \& Kjellberg, 1979; Lykken, 1967; Lang, 1985; Obrist, 1981). One of the aims of the present study is to test the possibility that HR during anticipation shows a qualitative change from predominantly deceleration to acceleration after the UCS intensity has become unpredictable.

In the present study it was decided to use a $3 \mathrm{sec}$ anticipation period. With such an interval, the $S$ can relatively reliably predict UCS onset and HR changes are less influenced by uncertainty about exact UCS onset during anticipation than with longer periods (Orlebeke, personal communication). It was hypothesized that the usual short latency deceleration after CS onset would be less, or even absent after the experimental manipulation in the experimental group, and that a stronger accelerative trend would be observed during the later part of the anticipatory period.

The present study aims at testing the hypothesis that unexpectedly increased pain produces more fear than pain of similar intensity that follows a predictable regular pattern. More specifically, it was hypothesized that subjective fear and autonomic fear indices during anticipation are higher in a condition that is characterized by some unpredictable sudden increases in painful stimulation to a high intensity level than in a condition of constant high intensity stimulation. The direction of the heart rate response during anticipation of the UCS is of special interest here: it was expected that HR acceleration during anticipation would be more likely after sudden unexpected increases in UCS intensity.

In order to test this hypothesis, two groups were formed: the experimental group received 17 painful stimuli of medium intensity, unpredictably alternated with 3 painful stimuli of strong intensity; the control group received 20 painful stimuli of strong intensity which followed a predictable pattern with respect to intensity because they were of constant objective intensity. This design offers the possibility of studying the effects of unpredictable, sudden increases in intensity per se, controlling for the effects of objective intensity of the stimulus. 


\section{METHOD}

\section{Subjects}

There were $26 \mathrm{Ss}_{3}$ all students, who participated voluntarily and received a small remuneration. There were 22 women and 4 men. Mean age was 20 yr (range $18-28$ yr).

\section{Materials}

Materials used were a Dutch version of the Eysenck Personality Questionnaire (EPQ, Sanderman et al., 1990), and $100 \mathrm{~mm}$ visual analogue scales (VAS) measuring predicted pain, experienced pain and fear of the next pain stimulus. The two scales pertaining to pain had at the left "no pain at all" and at the right "maximum pain" as anchors. The fear VAS had "no fear at all" and "maximum fear" as anchors. Subjects rated the VASs by placing a vertical mark. The ratings were measured in $\mathrm{mm}$.

\section{Apparatus and physiological recording}

The electric pain stimulation was produced by a Siemens Neuroton 627 via two Beckman $\mathrm{Ag}-\mathrm{AgCl}$ electrodes ( 8 diameter; $25 \mathrm{~mm}$ distance between the centres of the electrodes) attached to the $S$ 's right ankle. The electrodes were filled with Hewlett-Packard Redux Creme. The constant current AC stimulated produced a train of rectangular pulses. The stimulation was increased to the pre-selected level (described under Procedure) during $2 \mathrm{sec}$, remained at that level for $6 \mathrm{sec}$, and was gradually decreased to zero during 2 sec. Three seconds before the start of the pain stimulus, a slide projector projected a white rectangle on the wall for $1 \mathrm{sec}$ as a warning sigmal.

Subjects could press an escape button connected with a device for measuring reaction time to terminate the stimulation (see section 3.2).

Skin conductance level (SCL) and skin conductance response (SCR) were measured via two Beckman $\mathrm{Ag}-\mathrm{AgCl}$ electrodes $(8 \mathrm{~mm}$. diameter), placed on the medial phalanges of the second and third fingers of the $S$ 's non-dominant hand. The electrodes were filled with an isotonic paste and were connected with a Beckman Skin Conductance Coupler (type 9844), using the method of constant voltage (0.5 Volts). The skin was cleaned with distilled water.

Respiration was recorded from a Beckman Respiration Belt fastened around the $S$ " $s$ chest and connected to a Beckman Voltage/Pulse/Pressure Coupler (type 9853A), in order to detect SCRs due to respiratory irregularities.

Heart rate was measured via Beckman $\mathrm{Ag}$-AgCl electrodes filled with Hewlett-Packard Redux Creme. The electrodes were placed below the sternum, and at the left-hand side of the chest. The skin was clleaned with $70 \%$ alcohol. The electrodes were connected with a Beckman Voltage/Pressure/Volume Coupler (type 9853A). Before being transmitted to a microprocessor for heart beat detection, the signal was filtered through a low-pass filter (set at $40 \mathrm{~Hz}$.), in order to reduce noise due to the electrical pain stimulation.

A microcomputer (PDP Minc-11) controlled the experiment.

\section{Procedure}

Before entering the experiment room, $S$ s filled out the EPQ. On the basis of the Neuroticism score and sex, matched pairs were formed. The first $S$ of a pair was assigned to 
the experimental condition and was run first, the second $S$ was assigned to the control group. Ss were told that they were participating in a psychophysiological study on pain. After the $S$ had entered the experiment room and electrodes had been fastened, the objective stimulation level to be used was determined by increasing the level in steps of 0.4 $\mathrm{mA}$. In the experimental condition, the first painful intensity rated $>50 \mathrm{~mm}$ on the VAS measuring pain was chosen as to be used as the medium intensity level. For the high intensity stimulations the current was increased by $33 \%$ of the medium level. For the matched control $S$, the intensity was further raised in steps of $0.4 \mathrm{~mA}$ until the subjective pain rating was equal to or somewhat higher $(0-10 \mathrm{~mm})$ than the mean subjective pain score of the matched experimental $S$ at the heightened trials. All $S$ s were instructed that they would receive an (unspecified) number of painful stimulations, and that each stimulus would be preceded by the warning signal. Furthermore, the $S s$ were told that, although the objective intensity would remain constant, subjectively experienced pain might fluctuate; and that they could terminate the stimulus by pressing the stop button if they wished to do so. Subjects were instructed to complete the VASs after each trial. There were 20 trials. Four periods are distinghuished in each trial: $9 \mathrm{sec}$ pre-trial period, a $3 \mathrm{sec}$ anticipation period (starting with the presentation of the warning signal and ending with the start of the pain stimulus), a $10 \mathrm{sec}$ stimulation period, and a $9 \mathrm{sec}$ post-trial period. Fear of the painful stimulation was also rated before the first trial. Thus, 21 fear ratings and 20 responses of the other indices were obtained. Subjects in the control group received 20 shocks of the (high) level determined at the start of the experiment. Subjects in the experimental group received 17 shocks of the medium level, and 3 shocks of the high level at the 7 th, 12th and 17 th trials. The inter-trial intervals varied from 25 to $45 \mathrm{sec}$ (mean 39 sec).

In order to habituate the orienting response to the warning signal, 4 signals were given without being followed by a pain stimulus before the series of 20 trials began.

\section{Data definition, reduction and analysis}

The SCR to the warning signal was defined as the highest deflection starting during the $3 \mathrm{sec}$ anticipation period. SCR to the pain stimulus was defined as the highest deflection during pain stimulation, that is during 1 to $9 \mathrm{sec}$ after pain onset. SCRs were square root transformed (Levey, 1980). It should be noted that the anticipation and impact SCRs could be clearly distinguished, despite the somewhat short anticipation period, because impact SCRs started relatively late due to the fact that the pain stimulation level gradually increased from zero to its maximum during $2 \mathrm{sec}$. SCRs due to respiratory irregularities were replaced by estimated values, based on interpolation.

Changes in HR during anticipation (HRR $)$ were investigated by considering the last interbeat interval (IBI) before the warning signal and the three successive IBI's following that interval. In order to analyze the impact of the pain stimulus on HR, changes in HR during shock and after shock offset were analyzed using the mean pretrial HR as reference.

The responses to the warning signal and subjective fear of the next stimulus were averaged over the first seven trials (before any manipulations were carried out in the experimental condition), and over the last 13 trials. The averaged responses of the last 13 trials were analyzed by means of MANOVA. In case of subjective fear and SCRs the averaged responses of the first 7 trials were employed as covariates in order to reduce between $S$ variance. The HR changes during anticipation were analyzed by means of a trend analyses. The first and second order trends were inspected. In addition to univariate 
$F$ tests, the SPSS-X MANOVA output yields $t$-tests, which allow for directional interpretation. The between-group effect was tested one-tailed. The pain impact responses of trials 7,12 and 17 were averaged and compared between groups by means of MANOVA.

\section{RESULTS}

\section{Check of the experimental manipulation}

The selected medium intensity level of the experimental group (mean objective intensity $2.6 \mathrm{~mA}$, mean pretest VAS rating $54 \mathrm{~mm}$ ) was significantly lower than the selected high intensity level of the control group (mean objective intensity $3.4 \mathrm{~mA}$, mean pretest VAS rating $65 \mathrm{~mm}$ ): $t(24)=2.01, p<0.05$ (objective level) and $t(24)=4.02, p=0.001$ (subjective rating). The objective high intensity level was the same for the two groups $(t(24)=$ 0.26 ). Thus, it can be concluded that the experimental manipulation succeeded in selecting a high intensity level in the control group that was equivalent to the high intensity level of the experimental group.

\section{Immediate effects of the experimentally increased pain stimulations}

In order to test the immediate effects of the experimental manipulation (responses to the suddenly increased pain stimuli were expected to be larger than those to the stimuli of similar intensity in the control group, which were predictable), a MANOVA was carried out on the mean SCR, change in HR (expressed as the mean HR after pain stimulus onset minus the mean HR during the pre-trial period) and subjective pain ratings of trials 7,12 and 17 . There was a strong multivariate experimental effect $\left(F_{\text {wiks }}(3,22)=5.77, p=0.005\right)$. Univariate tests indicated that the change in HR, SCR and experienced pain of these trials were larger in the experimental group than in the control group $(p$ 's $<0.05)$. Detailed inspection of the HR revealed that two accelerations could be distinguished: an acceleration at the start of the painful stimulation, and an acceleration after shock offset. According to Turpin (1986), the short latency HR acceleration reflects a startle reflex, the long. latency acceleration a defense reflex. A separate analysis of these two HR accelerations revealed that only the short latency $H R$ acceleration was significantly stronger in the experimental condition $(t=3.57, p=0.001)$. The experimental effect on the long latency GR acceleration was marginally significant $(t=1.45, p=0.08)$. There was a significant higher frequency from the high intesnity pain stimulus in the expeirmental condition than in the control group $(p<0.05$, see section 3.2$)$. It can be concluded that the experimental manipulation succeeded in inducing stronger responses to the pain stimulus. In particular the startle response appeared to be stronger in the experimental group.

\section{Multivariate test of fear responses}

Subjective fear of the pain stimulus, as well as SCRs and HR changes to the warning signal during trials 8-20 were analyzed by means of MANOVA. There appeared to be a multivariate between-group effect $\left(F_{\text {willy }}(3,22)=5.00, p<0.01\right)$. The univariate tests are presented below. During trials $1-7$ (before any experimental manipulation) the multivariate between-group effect was $n . s .\left(F_{\text {wina }}(3,22)<1\right)$. 


\section{Subjective fear}

As is shown in figure 1, subjective fear of the pain stimulus was similar in the two conditions before the experimental manipulation $(a(24)=0.00, p=1.00)$. With the mean fear ratings during these trials $(1-7)$ as covariate, the between group effect on the averaged fear ratings of trials 8-21 was significant $(t(23)=2.21, p<0.02)$. Post-hoc tests revealed significant increases in fear in the experimental group at trial 8 (compared with fear of trial $7 ; t(24)=5.41, p<0.001$ ), at trial 13 (compared with fear of trial $12 ; t(24)=2.04$, $p=0.03$ ) and at trial 18 (compared with fear of trial $17 ; t(24)=1.85, p=0.045$ ). Therefore, these results support the hypothesis that fear of the pain stimulation would be increased by the sudden increases of intensity in trials 7,12 and 17, and that fear reduction would be slower in the experimental condition due to these increases.

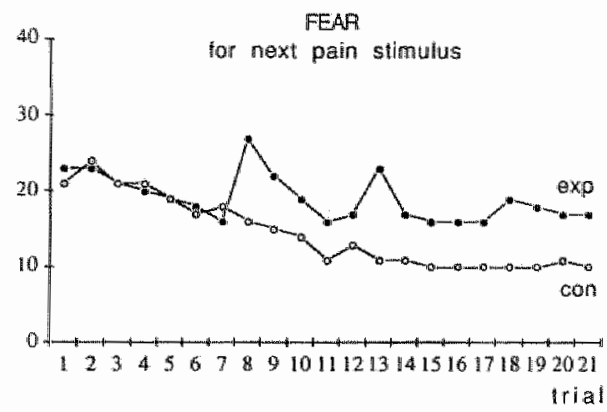

Figure 1. VAS ratings in mm. of subjective fear of the pain stimulus on a trial-by-trial basis. In the experimental condition, the pain stimulation level was unexpectedly increased at trials 7,12 and 17 . The experimental $S \mathrm{~s}$ show higher subjective fear during trials 8-21 than the control $S$ s $(p<0.02)$.

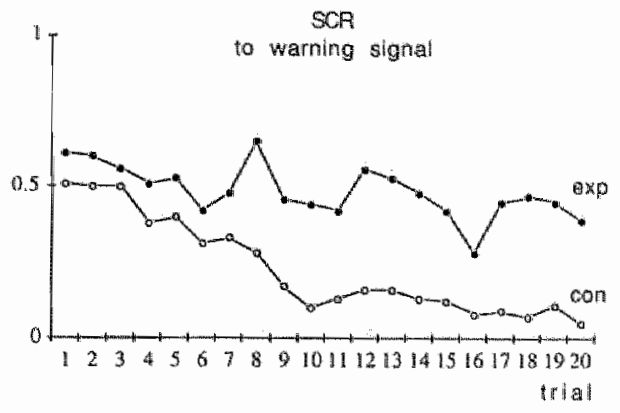

Figure 2. Anticipatory SCRs (in microSiemens $s^{1 / 4}$ ) following the warning signal that preceded the pain stimulation. During trials 8-20, the SCRs were significantly higher in the experimental condition than in the control group $(p<0.001)$.

\section{SCRs during anticipation}

During trials 1-7, the difference between the groups as to the SCRs following the warning signal was n.s. $(t(24)=0.83, p=0.41)$. When the mean SCR of trials 1.7 is employed as a covariate, the mean anticipatory SCR of trials 8-20 is larger in the experimental group than in the control group (figure $2 ; t(23)=3.62, p<0.001$ ). Note that the SCRs decreased to almost zero in the control group, despite the fact that this group received more stimuli of high intensity. The three increases in pain intensity were all followed by anticipatory SCRs increased in comparison with the anticipatory SCR in the previous trial (collapsed over the three trials: $t(12)=2.10, p<0.05$ ), but the effect becomes smaller later in the series (trial 8 ws $7: t(12)=1.97, p<0.05$; trial 13 vs 12 : $t=1.64, p=0.06$; trial $18 v s$ 17: $t=1.14, p=0.14$ ). These results support the hypothesis that unpredictable increases in pain are followed by increased anticipatory SCRs and lead to less extinction of these SCRs. 
Figure 3 (left) illustrates the mean heart rate deceleration after the warning signal in both conditions during trials $1-7$, reflected by a significant second order trend $(t(24)=$ $-2.20, p<0.05)$. The between-group differences were n.s. $\left(t(24)\right.$ 's $\left.<1, p^{\prime} s>0.60\right)$. As is shown in the right part of figure 3 , the heart rate changes after the warning signals of trials 8-21 were accelerative in the experimental condition, in contrast to the responses in the control group, which remained decelerative as indicated by a significant difference in the first order trend $(t(24)=1.92 ; p<0.05)$. The large fluctuations, and the small $N$, did not allow for detailed inspection of the heart period responses in separate trials. Summarizing: HR changes after the warning signal support the hypothesis, and suggest that conditioning of fear to a warning signal, as indicated by $H R$ acceleration, is more likely in conditions with unpredictable intensity of the UCS, than in conditions with predictable intensity. In order to test the possibility that only $S s$ who escaped from the pain stimulation showed anticipatory HR accelerations, the HRRs of $S s$ who escaped once or more were compared with the HRRs of $S s$ who did not escape. The difference was n.s. when the comparison was made over both conditions $(t(24)=1.05)$. Within the experimental condition, $S$ s who escaped once or more did not show HR accelerations, but $S$ s who did not escape reacted with HR accelerations to the warning stimulus (mean IBI decrease $=$ $0.013 \mathrm{sec}$. between effect, $t(12)=1.80, p=0.10)$. Thus, the HR acceleration in the experimental condition could not be attributed to actually escaping from stimulation.

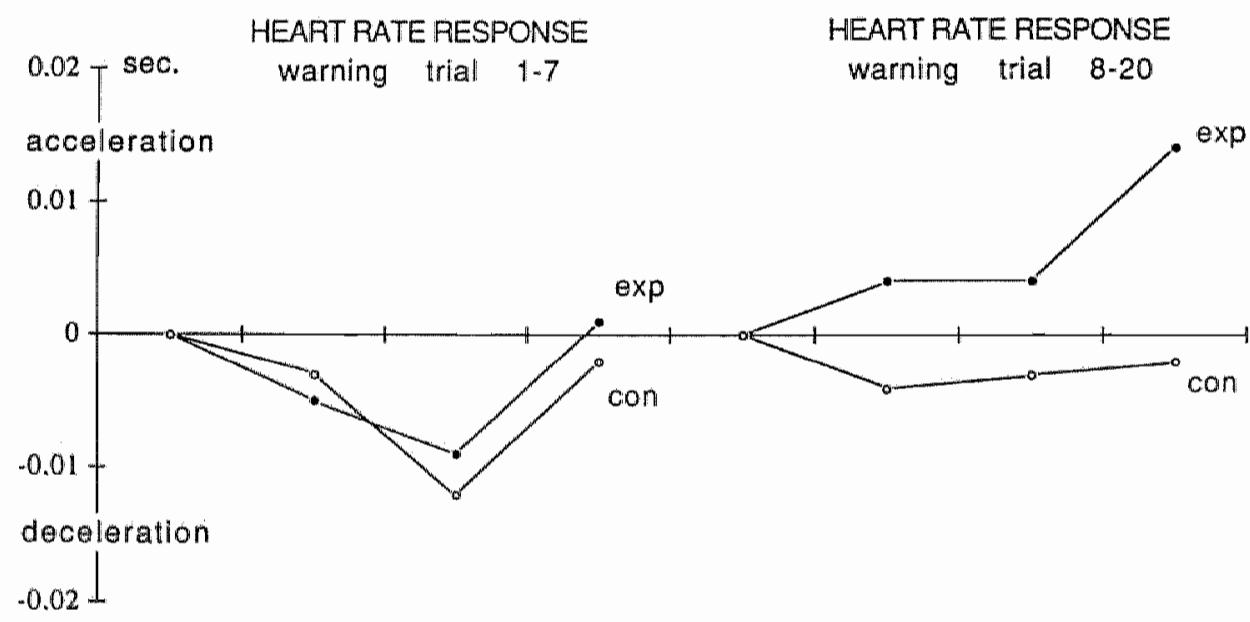

Figure 3. (Left) Heart rate response to the warning signal during trials 1-7. Both conditions showed decelerations. The figure depticts the inter-beat interval of three beats following the signal, compared to the interval preceding the signal.

Figure 3. (Right) Heart rate responses to the warning signal during trials 8-20. The control Ss still responded with HR deceleration, but the experimental Ss showed HR acceleration $(P<0.05)$.

\section{DISCUSSION}

The present study clearly supports the hypothesis that sudden increases in intensity of a UCS contribute to fear of the UCS. Subjective fear and physiological responses were 
larger in the condition in which a medium-intensity UCS was unpredictably alternated with a high-intensity UCS than in the condition in which all stimuli were predictably strong. In the case of subjective fear and SCRs, the clearest long-term effect appeared to be that the reduction of these responses was delayed. In the case of the HRRs, it was rather a qualitative difference that emerged: in the condition with unpredictable intensity, HR acceleration after the waming stimulus was found, in the condition with predictable intensity HR deceleration.

It seems unlikely that these effects should be attributed to other between-group differences. The Ss were carefully matched on the basis of their sex and neuroticism scores; the objective stimulation level in the experimental condition during the 17 medium-intensity trials was significantly lower than the level in the control group, while the experimental trials $7,12 \& 17$ had equal objective intensity in both groups; subjective pain levels were significantly lower in the experimental condition than int he control condition before the experimental manipulation took place; and changes in HR after the warning signal were similar in both groups before the experimental manipulation took place. Because of this, it can be concluded that it were the sudden increases in intensity that caused the stronger fear reactions.

The different changes in HR to the warning stimulus are intriguing. The control group displayed HR decelerations after the warning signal, as did the experimental $S$ s before any experimental manipulation had taken place ${ }^{2}$. Therefore, the observed HR accelerations in the experimental $S s$ should be attributed to the sudden increases of the pain stimulation, rather than to its intensity per se. This finding suggests that unpredicted sudden increases in intensity of an aversive UCS might be an important factor in the development of HR accelerations to a CS. The different reactions suggest that two different forms of preparation for the UCS develop, depending on the predictability of the UCS. Under conditions of high predictability (high certainty), an orienting-like preparation seems to take place (SCR and HR deceleration, cf. Lykken, 1967), whereas under conditions of unpredictability (high uncertainty about the UCS), a defensive attitude seems to predominate (large SCR combined with HR acceleration, Lykken, 1967). This interpretation suggests that under conditions of high certainty about the stability of the (tolerable) UCS intensity, the organism prepares itself by adopting something like an attitude of acceptance, whereas uncertainty about the UCS intensity may lead to a more defensive, rejecting attitude.

Heart rate acceleration has also been interpreted as a remnant of flight/fight behaviour which is not put into action (Obrist, 1981; Lang, 1985). Flight/fight reactions are usually associated with increased sympathetic drive, which takes appr. $2 \mathrm{sec}$ before HR is affected. Thus, in the present experiment possible differences between experimental and control group in this respect can only be observed during the last beat. Note that after the unpredictability manipulation the last beat in the anticipatory period showed the largest acceleration. Interpreted in this way, the present findings indicate that people prepare for an aversive UCS by means of activation, reflecting a tendency to or a preparation for a flight/fight response, under conditions of unpredictability of the important aspects of the UCS. In yet another view, HR acceleration as a reaction to a CS indicates that Ss learned to respond emotionally to the CS; whereas HR deceleration indicates (non-emotional) attention to the CS (Lang, 1985). In this view, decelerators also learn about the CS-UCS relationship (which is indicated by the relatively small SCRs and HR deceleration), but

\footnotetext{
The reduced $H R$ deceleration during anticipation of trials 8-20 in the control group is probably caused by habituation (cf. Bohlin \& Kjellberg, 1979).
} 
only those whose HR accelerates show a transference of negative evaluation from UCS to CS (Lang, 1985, p. 153). The heart rate responses to the pertinent pain stimulations (trials 7, 12 and 17) suggest that the startle response (indicated by the short latency HR acceleration during the unpredictedly increased stimuli) in particular contributed to the development of the responses to warning stimuli. Therefore, the immediate effect of a UCS that is suddenly stronger than expected seems to produce a startle response, which contributes to the development of fear and a different anticipatory response to the CS in the experimental group.

It seems difficult to explain the differences in anticipatory HR by means of the stimulus substitution rule. According to this rule, the result of classical conditioning is that the CS comes to eliciti a subset of the responses that were originally elicited by the UCS (Gray, 1975). Because post-hoc analysis revealed that both groups reacted to the UCS with a short latency HR acceleration (during the start of the pain stimulus) as well as with a long latency HR acceleration (after shock offset) in both phases of the experiment (trials 1-6 and trials 7-20), HR accelerations as a reaction to the waming signal could have been expected. Heart rate deceleration appeared to be the rule, however, and HR acceleration the exception caused by the manipulation. Modern Pavlovian conditioning theory might be more capable of explaining the different HR responses. According to this theory, Pavlovian conditioning is no longer considered mechanistic learning of S-R reflexes (Davey, 1989). The CS is considered to elicit an internal representation of the UCS, which is evaluated in turn. This evaluation results in the final responses to the CS. Thus, the responses to the CS reflect the anticipation of the coming UCS as its cognitive representation is evaluated. It seems obvious that unpredictable sudden increases in the intensity of the UCS can be an important aspect of the cognitive representation of the UCS which leads to a more negative (distrustful) evaluation of the UCS, and consequently to a more defensive (possibly flight/fight) kind of preparation for the UCS, compared to the preparation for a predictable UCS. It should be noted, however, that the present study did not examine responses to the CS during extinction. Further studies are needed to investigate this issue.

Another limitation of the present study should also be mentioned. Whereas the present findings indicate that sudden unpredicted increases in intensity of an aversive UCS might contribute to acquired fear, it is unclear whether comparable increases would have similar effects if they would be made predictable by signalling the increase. It is conceivable that sudden deviations from a regular pattern characterizing an aversive UCS whether signalled or not, might lead to the development of stronger autonomic anticipation responses and increased subjective fear, possibly via dishabituation (note that in the present study the experimental trials 7, 12 and 17 caused stronger impact responses). Thus, predictability in the sense of signalling might be ineffective. But predictability in a more formal sense, pertaining to the certainty inherent to regularity of a stimulus, might still be important. Clearly, a study comparing the effects of signalled $w s$ unsignalled sudden increases in UCS intensity is indicated. ${ }^{3}$

The effects of unpredictability of intensity open up the possibility that other aspects of predictability might also be important. The most obvious candidate seems to be duration of the aversive UCS, but other aspects might also be important (see for instance Imada \& Nageishi, 1982). It would also be interesting to investigate the effects of unpredictability of UCS intensity on conditioned UCR diminution. It is possible that conditioned UCR

${ }^{3}$ See section 2.3 for such a study. 
diminution only takes place under conditions of high predictability of the UCS. The present study cannot answer this question, because an adequate control group (a group that receives predictable UCSs of medium intensity) is lacking. Nevertheless, the (post-hoc) findings that SCRs to the pain stimulus in trials 8-11,13-16, and 18-20 were significantly larger in the experimental group than in the control group (but were comparable during trials (1-6), and subjective pain ratings of the experimental Ss were no longer significantly smaller during these trials than the ratings of the control $S$, are suggestive. 


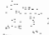

$\therefore \quad$

$\therefore$ 


\section{CHAPTER 4. INFLUENCES OF CONTROL, ATTENTION AND EMOTIONS ON PAIN}

Chapter 4 addresses the influences of control, attention and emotions on pain. Section 4.1 offers a review of experimental and clinical research on the effects of perceived control over pain. The question is addressed whether perceived control reduces the experience of pain and whether it has also a positive effect on other pain variables. It is concluded that control can, but not always does reduce pain reactions, depending on the type of pain reaction (pain tolerance and endurance are most clearly influenced) and on the type of control (controlling responses by which the $S$ can keep pain intensity or pain duration below unbearable limits have the strongest effects).

The question is also addressed whether the suffering of chronic pain patients can be understood as resulting from their being in a state of learned helplessness caused by a lack of (perceived) control over pain and life in general. This discussion leads to a hypothetical model of the relationship between chronic pain and low perceived control. Finally, possible mechanisms underlying positive effects of perceiving control over pain are discussed. From this discussion a number of hypotheses follow. Three are relevant for the experimental studies reported in this chapter. The first (executing control over pain might be a positive experience per se which can compensate pain) and the second (being able to control essential aspects of a painful stimulus increases certainty that the stimulus will not become unbearable, which allows the $S$ to distract attention from pain) are further explored in section 4.2. The third (anxiety does not influence pain., but attention does) is addressed in sections 4.3 and 4.4 .

In section 4.2 the interaction between mood (more specifically, the elated-depressed mood dimension) and control over pain is investigated. If being able to exercise control over pain induces a positive mood, and failing to exercise control over pain induces a negative mood, and the effects of control on pain reactions are mediated by these moods, it follows that these moods per se should be able to influence pain reactions, and that when these moods are manipulated independently from controllability, the effects of control should be influenced by the mood manipulation. This hypothesis is put to a test in a $2 \times 2$ design with mood (elated $v s$ depressed) and control ( $w s$ no-control) as independent factors. In this experiment the hypothesis is also tested that mood influences the effects of control through influencing perceptions of control. Finally, the hypothesis is explored that certainty about how much pain can be expected mediates the positive effects of control. In short, the results indicate that certainty mediates the effects of control on physiological pain responses, but not on the experience of pain. There are indications that not the elated/depressed emotional dimension, but the dimension of anger mediates the influence of control on the experience of pain. Lastly, though mood influenced the ratings of perceived control, these mood-congruent changes in ratings of control did not affect pain reactions.

In sections $4.3 \& 4.4$ the influences of anxiety and attention on pain are the matter of interest. A critical analysis of the lack of convergence between previous empirical studies on the influence of anxiety on pain leads to the hypothesis that the influence of anxiety on pain is for the major part mediated by the focus of attention. Depending on the relevant features of the anxiety manipulation, attention might be directed towards, or away from 
pain, thereby influencing pain reactions. Such a theory might also explain contradictory clinical findings: sometimes pain is observed to be increased when the $S$ is anxious, sometimes it is observed to be decreased, probably because in the first case anxiety motivates the $S$ to direct attention away from pain, and in the second case towards pain.

Two experiments are reported in which the focus of attention is manipulated independently from the level of anxiety. The attentional hypothesis is strongly supported by the subjective pain reports, but the physiological pain responses give less evidence for the hypothesis (though neither of the two traditional hypotheses, which state that anxiety either increases or decreases pain responses, was clearly supported). Thus, attentional focus is probably a major factor mediating the influence of anxiety on the experience of pain. 


\section{SECTION 4.1. PERCEIVED CONTROL AND THE EXPERIENCE OF PAIN}

\section{INTRODUCTION}

Pain can have uncontrollable aspects. Not only medical treatment, but also traditional ways of dealing with pain (taking rest, avoidance of activities) can fail to produce pain relief. Sometimes, pain results from medical treatment or from natural processes, such as childbirth, which are largely beyond the influence of the subject. Although it may seem self-evident that the experience of having no control over pain is a source of suffering in itself, the question can be posed whether having no control intensifies the experienced pain itself, and conversely, whether experiencing control over pain can diminish the pain experience. Besides the possible direct relationship between control and pain, the long-term consequence of failing to acquire control over prolonged pain is of interest, since many patients have to deal with chronic pain. Some chronic pain patients seem to suffer in a manner that is disproportional to medical findings and display very helpless behaviour, whereas others lead fairly undisturbed lives. As will be seen, these differences may be explained by the lack of control and the associated learned helplessness experienced by the former group. Before discussing the relationship between controllability and pain, two general issues are treated. First, what is meant by "perceiving control"? Second, what is pain and why can psychological factors influence it?

\section{The perception of control}

Very generally, control can be defined as some behaviour (overt or covert) that reliably changes something else. Thus, when a subject is exerting control over pain, the subject behaves in a certain way that alters the pain experienced. In this chapter, control is largely taken to imply ways of decreasing, limiting or preventing aversive experiences such as pain. Thus, its use is confined to the cases where control has positive consequences. Interestingly, various studies have shown that actual control does not have any effect in the absence of the perception of a reliable relationship between the controlling response and its outcome. Control can be perceived even when it is not actually present, but may still have the same effect as real control. Thus, the belief that pain can be controlled may have positive consequences, even when the controlling response is not used. What is important, then, is the perception of control.

\section{The experience of pain}

Pain can be defined as "an unpleasant sensory and emotional experience, associated with actual or potential tissue damage or described in terms of such damage" (IASP, 1986). This broad definition does justice to the many aspects of pain. Nociception, the sensory detection, transduction, and neural transmission of noxious events forms the basis for pain sensation. It is doubtful whether, even in the most controlled laboratory condi-

${ }^{1}$ Reprinted with permission from Wiley \& Sons Ltd; Arntz, A. \& Schmidt, A., Perceived control and the experience of pain. In: Steptoe, A. \& Appels, A., Stress, Personal Control and Health, $1989,131-162$. 
tons, a linear relationship is demonstrable between peripheral, nociceptive activity and the pain experienced, because the nociception can be modified strongly at low levels within the nervous system. (Wall \& Melzack, 1985). This means that the signal that finally leads to the awareness of pain can be amplified or reduced by higher processes. Nociception and the experience of pain can even be totally unrelated: pain can be reported without nociception, as in phantom pain (Sherman et al., 1987); or there can be noxious stimulation without pain sensations (Wall, 1979). Assuming that pain is a subjective sensory and emotional experience, it is clear that pain can be modified to a high degree by influencing factors such as the extemal environment or the focus of concentration (Sternbach, 1978). Thus, psychological factors play a very important role in the experience of pain. Analogous to (other) emotions, pain has various aspects that are not necessarily strongly interrelated: since subjective-emotional, cognitive, behavioural and physiological reactions can be distinguished.

\section{Conclusion}

A relationship between controllability and pain can be postulated on theoretical grounds. Having established this, two main questions can be raised. First, does perceiving control actually diminish pain? Second, if this is the case, why does perceived control have this influence? In addressing the first issue, a distinction should be made between various kinds of pain. The first category that will be discussed pertains to laboratory pain. It must be separated from naturally occurring pain, because its validity with respect to clinical pain may be questionable. However, studies of laboratory pain do allow the researcher to control pain stimulation in a systematic fashion. As will be seen, elegant experiments have been done on the influence of control over laboratory pain. In addition, pain coping strategles have been extensively studied in the laboratory, and the influence of perceived control on the efficacy of these will also be discussed.

The second category is acute clinical pain, which must be distinguished from chronic clinical pain. The lower boundary for chronicity is usually taken to be 6 months. It is generally accepted that the distinction between acute and chronic pain is not only of diagnostic value but also has far-reaching therapeutic consequences. It will be argued that, on balance, despite the differences between various kinds of pain, the first question can be answered in the affirmative. Finally, the last section dleals of the chapter outlines various explanations that can be offered to explain this effect.

\section{DOES PERCEIVED CONTROL REDUCE THE EXPERIENCE OF PAIN?}

\section{Does perceived control reduce the negative effects of laboratory pain?}

As early as 1949 (Haggard, 1949) positive effects of having control over a painful stimulus upon reactions to pain in the laboratory were reported. Previous reviews on the effects of control over aversive stimuli in general have come to different conclusions. Averill (1973) and Thompson (1981) concluded that controllability does not reliably decrease the impact of aversive events. However, both reviews used broad definitions of control, which may have obscured positive effects of control defined in more restricted ways. Miller (1979) applies a more restricted definition of control and concluded that controllable aversive events may have less negative effects. In contrast with these reviews, this section is restricted to control over noxious, painful stimuli. Animal research, where the subjec- 
tive experience of pain cannot be assessed, is not discussed. In this section, the question will be addressed whether behavioural control over the pain stimulus itself reduces the effects of laboratory pain. Controllability of aversive events is here defined as the availability of an overt response that actually modifies the event, or the perception of or belief in the availability of this response. Different modifying responses have been object of research, e.g. escape from the event, changing its probability of occurrence or self-administration of the event, etc. (cf. Miller, 1979).

\section{Experimental studies on controllability and pain: types of control and designs}

As Miller (1979) has argued, several different types of behavioural control can be distinguished. The first is selfadministration (SA), where subjects administer the painful stimulus to themselves, in contrast to administration by the experimenter. The second type of control is instrumental control (I), where the subject can (partially) avoid, escape or reduce the stimulus by some response (actual control); or believes that he or she can do so, but objectively cannot because the experimenter controls the stimulus (perceived control). In this class of experiments, the subject can, for instance, terminate the shock. In the actual control design, the group that has no control is a yoked one (every subject in the no-control group is yoked to a subject in the experimental condition so that the total amount of aversive stimulation is equal). The perceived control design is similar, except that the experimenter determines the amount of aversive stimulation, so each pair of subjects experiences the same stimulation. The third type distinguished by Miller is 'actual control equated for predictability "(CP). Predictability can be defined as the availlability of a sign that reliably signals the start, end, increase or decrease of the noxious stimulus. Predictability has been found to influence reactions to painful stimuli, and in many experiments the experimental manipulation of control is confounded with effects of predictability. For instance, when the subject can reliably stop a painful stimulus, the subject can also reliably predict when it stops. The subject that has no control, cannot predict the end of stimulation. Therefore, designs have been developed where predictability is controlled for (for instance by providing an external cue that signals when the stimulus stops in both conditions - the only difference between conditions is that subjects in the control condition believe that they caused the termination of the stimulus, whereas subjects in the no-control group do not believe this belief). The fourth type, potential control (P), refers to conditions where subjects believe or know that there is a controlling response available but they do not use it. Examples are studies where subjects are told that they can withdraw their hand out of the ice water, but are (sometimes emphatically) asked not to do so. There is also another design, not mentioned by Miller. In the loss of control design, subjects are first given control and later on deprived of it. This group is contrasted to a group that never had had control (e.g., Staub et al., 1971, Exp. I).

\section{Indices of effects of controllability}

Different indices of effects of controllability have been investigated. In the present context, the following will be discussed: subjective and physiological distress during anticipation; subjective and physiological impact of the painful stimulus; tolerance/endurance of the painful stimulus; and postexposure performance. Following Miller (1979), skin conductance responses (SCR) during anticipation and impact, as well as heart rate (HR) during impact are summarized here as most important physiological indices. Although not strictly an effect of controllability preference (subjectively expressed or actual choice) for control will also be discussed, since it highlights an important aspect of control. 


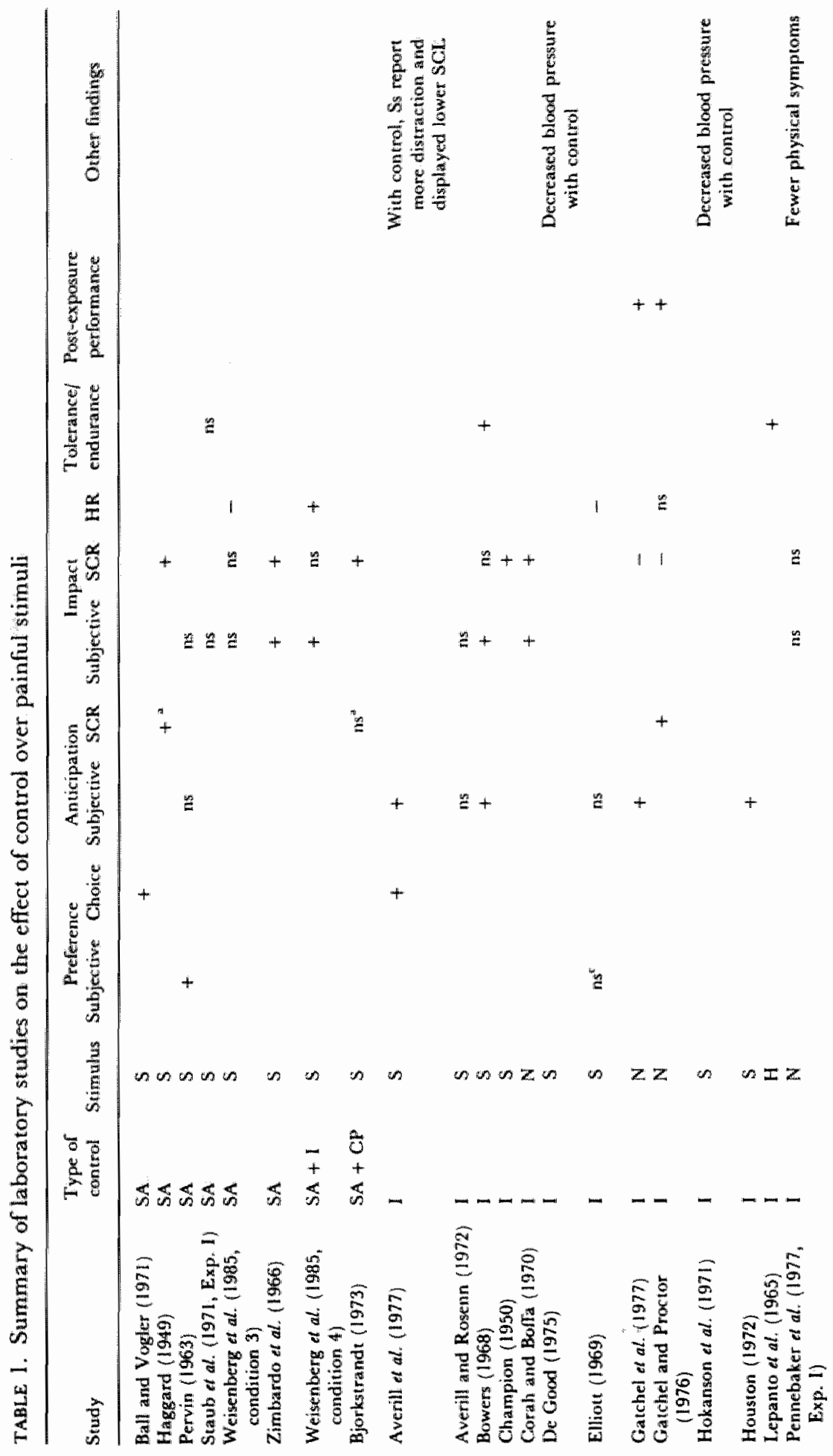




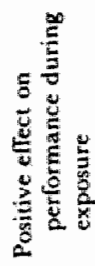

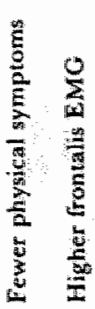

定

$\stackrel{m}{=}+1+\infty+\infty$

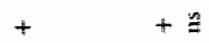

$z$ in on non zzzz

$\sin$

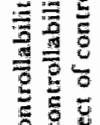

总总若

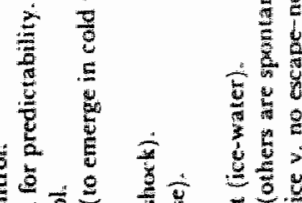

- - - ठิठีย

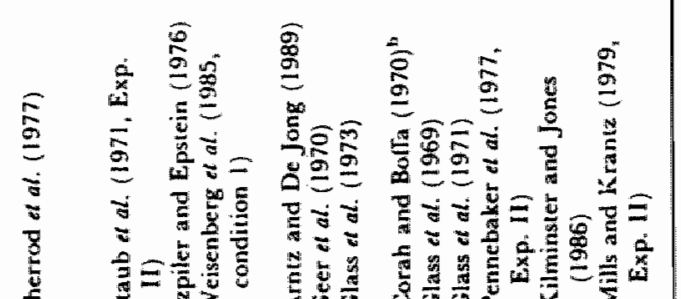

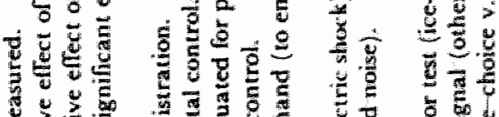

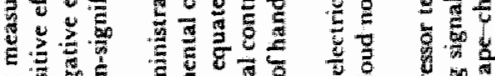


Table 1 summarizes the findings of 34 studies with respect to these indices. The studies are clustered according to the type of control.

\section{Selfadministration}

Eight studies investigated the effects of self-administration contrasted with administration by the experimenter (table 1). Although preferred, self-administration does not appear to have a powerful positive effect on anticipation and on pain. However, it does not have negative effects.

\section{Instrumental control}

Instrumental control was defined as the subject"s perception that he or she avoids, reduces or stops the painful stimulation by an overt response. Results of 17 studies (table 1) indicate that instrumental control reduces anticipatory distress, increases tolerance/endurance of pain and fosters post-exposure performance. The impact of painful stimuli can be less distressing when the subject has control. However, very difficult responses or high uncertainty about the outcome of the response may lead to less positive and even negative effects of controllability, especially on physiological impact reactions (cf. Miller, 1979). For instance, the negative effect of control on SCR in the Gatchel studies has been attributed by Miller (1979) to the fact that subjects had to learn what the escape response was. In the present context, subjective impact is an important issue. Only one study (Weisenberg et al., 1985, condition 1) reports a negative effect of control on experienced pain. The study has, however, several methodological shortcomings (no pre-test to assess subjective pain level; a small number of trials where control could be employed; a weak manipulation of control; and an unclear definition of the controlling response - the publication does not describe it). The most conservative conclusion, then, is that controllability is certainly not worse than no control with respect to subjective pain and is superior with respect to tolerance.

\section{Control equated for predictability}

Theoretically, this design is very important since it differentiates the effects of instrumental control over the end of the noxious stimulation from effects of predictability. There are, indeed, indications that control can have positive effects above predictability on experienced pain, and on physiological reactions during anticipation and impact (table 1).

Does the difficulty of the controlling response influence the effects of control on subjective pain? This question was addressed in a recent study by Arntz and De Jong (1989). Subjects had to perform IQ-test like tasks in two series, each task followed by painful electric shock. In the perceived-control condition, subjects were told that they could decrease the duration of the shock in the second series from 6 seconds to 3 seconds by giving the right answer. Actually, they always received shocks of 3 seconds. Subjects in the no-control condition also performed this task, but they were simply told that shock duration would be reduced irrespective of their performance. Thus, subjects in the nocontrol condition had equal or even higher predictability of the stopping of the stimulus than subjects in the perceived control condition (who believed that shock duration would only decrease if they choose the right answer).

Figure 1 illustrates the results on subjective pain (corrected for pretest level).

Subjects in the perceived control condition rated the shocks significally less painful than subjects who did not believe that they could control shock duration. This study illustrates that even difficult controlling responses can lead to reduced pain experience. 


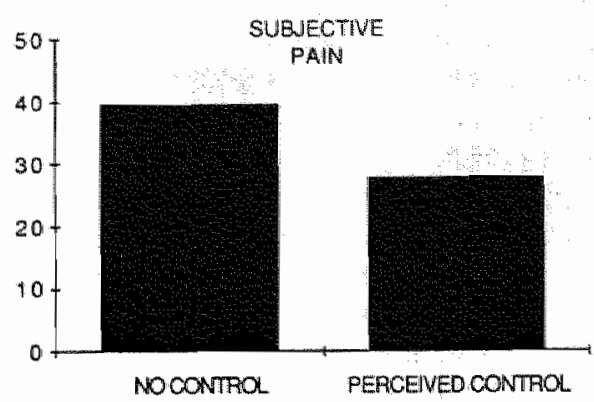

Figure 1. Subjective pain with and without perceived control over the termination of painful electric shock $(p<0.05$ ). (Arntz \& De Jong, 1989)

\section{Potential control}

With potential control, subjects clearly tolerate more pain and perform better after pain exposure (table 1). It is, however, notable that subjective pain is not consistently influenced by potential control. Physiological data sometimes indicate no effects of potential control. There are, however, methodological problems with the potential control design. The effect of controllability depends, of course, on the actual and perceived differences between potential control and no-control conditions. Subjects are with varying degrees of emphasis asked, or even instructed, to refrain from using the controlling response. Thus, the experienced controllability can be limited by strong experimenter demands. Sometimes subjects who use the response are replaced by new subjects (e.g. Corah \& Boffa, 1970). In the cold pressor test studies, subjects always have the option to withdraw their hands from the ice-water, giving them considerable potential control. Thus, clearer results may be found when the difference in controllability between conditions is maximized.

\section{Loss of control}

One study investigated the effect of loss of control (Staub et al., 1971). Subjects who could control increase in shock intensity were later on deprived of control. Compared with the control group who had never had control, they rated pain higher and showed lower shock tolerance. This study indicates that loss of control may be even worse than never having had control.

\section{Conclusions}

The question whether perceived control reduces negative effects of laboratory pain can be answered with a qualified yes: perceived control can, but not always does, reduce the negative effects of laboratory pain. Its effects depend on a number of factors, including the effect measure, the type of control and its salience.

Concerning the different measures, the dissociation between physiological, subjective and behavioural indices is remarkable. On the behavioural level, unequivocal positive effects are found on pain tolerance/endurance and on post-exposure performance. Physiological anticipation effects indicate that, with control, anticipatory distress is equal to or less than without control. Physiological impact data show equiwocal results, with the negative effects of control possibly depending on uncertainty of the controlling response in some studies. Nevertheless, there is a tendency towards a positive effect of having control on physiological impact reactions. At the subjective level, the effects of control on anticipation and impact appear to be equal to or less than those experienced without control: if 
control does not do any good, it does not do any harm either. In the present context tolerance/endurance and subjective pain are most important: people are more disturbed by their experience of pain than by physiological reactions. Therefore, our conclusions with respect to the effects of control on laboratory pain must be more positive than those of Averill (1973) and Thompson (1981).

The effects of control appear to vary with different types of control. Control over the initiation of a painful stimulus has only a limited positive effect on the impact of the stimulus. In contrast, control over the end of stimulation or over increase of noxious stimulation has more powerful positive effects. The effects on tolerance are most clear: when the subject can terminate the stimulus or control its maximum intensity, tolerance and endurance are higher. This finding is of great clinical importance in itself, for in clinical pain, tolerance and endurance of experienced pain influence to what extent patients can function normally or see themselves as ill, disabled, in need of medication or restrict$e d$. Theoretically, this observation indicates why control can have positive effects on the experience of pain. We will return to this issue later.

The positive effects of perceived control clearly depend on the degree of certainty the subject has that the behavioural response will have an effect.

Thus, Averill (1973) and Miller (1979) conclude that only when there is high certainty that the controlling response will be efficacious do positive effects of perceived control emerge.

In conclusion, the inference that "if control does not do any good, it does not do any harm either" could be more positive if experimental studies were more rigorous methodologically. First, the intensity of the pain stimulus should be individually adjusted, since subjects differ enormously in experienced pain intensity. Without controlling the individual level, inter-subject variance can obscure the effects of control versus no-control. Second, predictability should be controlled. Third, only behavioural responses that have a salient relationship with their presumed effects should be used. Fourth, the differences between control and no-control manipulations should be maximized (which is certainly not the case in cold pressor test studies). Last, and most important: the positive effects of having control depend on the subject's perception that he or she can limit or terminate the noxious stimulation. Therefore, experimental manipulations should concentrate on these aspects.

\section{Pain coping strategies and perceived control in the laboratory}

If the source of pain cannot be controlled, experience of pain seems to be inevitable. However, it has been shown that various pain coping strategies can be employed to promote pain endurance and to reduce suffering. In the following, the role of perceived control over the application of such strategies will be discussed. In this context, perceived control is defined as the perception that the coping methods can be applied during pain, and will have positive effects on the subjective pain experience, on pain tolerance or on pain endurance.

\section{Pain coping strategies}

A variety of pain coping strategies have been investigated in the laboratory: relaxation, distraction, positive self-statements, imagery strategies, hypnosis, stress inoculation training, cognitive transformation of the situation, etc. Research comparing several strategies indicates that vivid distracting mental activity (via internal or external stimuli) is a superior strategy (Kanfer \& Goldfoot, 1966; Grimm \& Kanfer, 1976; Worthington, 1978; Beers \& Karoly, 1979; for reviews: Tan, 1982; Turk et al., 1983). Investigation of spontaneous 
coping strategies also indicates that wivid distraction is most powerful (Rosenbaum, 1980). Tolerance is found to be the most sensitive parameter, while pain threshold and subjective discomfort seem to be less influenced by these strategies.

\section{Perceived control and pain coping strategies}

Several forms of control that the subject can exercise over the application of coping strategies have been investigated. In general, from this research it can be concluded that perceiving control over the practice of the strategy is a powerful factor, and is probably a necessary condition for demonstrating its effectiveness.

To begin with, various kinds of choice are found to have positive effects. Choice of coping strategy enhances pain tolerance compared with prescribed strategies (Avia \& Kanfer, 1980). Choice of elements of the coping method is also important. The effects of vivid imagination of distracting scenes on pain tolerance and on subjective pain were higher when subjects could choose the content of what should be imagined, compared to prescribed content (Worthington, 1978). It is interesting to note that subjects who chose the imagery content had more vivid imagery, and that vividness correlates with increased tolerance $(r=41)$ and decreased subjective pain $(r=-.30)$.

Personal influence over the timing of external distraction has also been found to be important: subjects who could advance distracting slides at their own rate tolerated cold pressor pain longer than yoked volunteers (Kanfer \& Seidner, 1973).

These findings might be attributed to the more powerful distracting properties of selfchosen and self-paced stimuli, rather than perceived control over the strategy . However, several studies have found that perceived control itself is important. In a second experiment, Kanfer and Seidner (1973) showed that contingent positive reinforcement of a coping response enhanced cold pressor tolerance more than mere distraction or non-contingent positive reinforcement. Thus controllability of reinforcement (controllability defined in terms of learning theory as contingency between response and reinforcement) of a pain coping response enhances pain tolerance. It can be concluded that control over reinforcement of coping fosters the belief in the ability to self control the coping strategy.

Comparable findings are reported in studies employing bogus feedback about pain coping techniques. Positive bogus feedback about relaxation performance as a coping response for cold pressor pain has been shown to increase pain tolerance (Neufeld \& Thomas, 1977). Since physiological arousall and subjective discomfort were not different between conditions, the authors concluded that the increased tolerance was not caused by more effective relaxation, but was actually caused by the subjects" belief in their ability to apply the technique. Positive bogus biofeedback about hand-warming ability has also been shown to increase cold pressor pain tolerance, as compared with negative bogus biofeedback (Litt, 1988, Exp. II). Interestingly, subjects preferred termination of the cold pressor. test to be contingent on hand-warming performance only when they were led to believe in high ability. It is unlikely that success $v$ failure feedback per se causes different pain tolerances; bogus feedback about ability to cold pressor pain did not reliably affect subsequent tolerance in a study by Litt (1988, Exp. I). In addition to perceiving controll over the use of a coping technique, subjects have to believe in its pain-decreasing properties: when told the rationale of technique, volunteers benefitted more from a pain coping technique than when they were asked just to apply it as a task (without a rationale) during the experiment (Girodo \& Wood, 1979).

\section{Atribution and pain coping}

As we have seen, perceiving pain coping strategies as effective and under voluntary 
control is essential. This can be restated in attributional terms: the person has to perceive a stable, internal source that carn positively influence the pain experience. Attributional research has further substantiated this view. Davison and Valins (1969) gave subjects a 'drug' and had them believe that it decreased the pain of electric shock by manipulating the shock level. Half of the subjects were told that they were given a placebo: these people subsequently tolerated higher shock levels than the those who believed that the drug had caused the pain relief. It can be concluded that experimental subjects attributed pain relief to their own pain coping abilities (a stable, internal source) and that this attribution promoted pain tolerance.

External, but controllable attribution can also promote pain tolerance. Nisbett and Schachter (1966) led subjects to attribute the physiological symptoms of painful shock to a (harmless) drug. These people then tolerated higher shock levels than controls. It seems that participants perceived the drug-induced symptoms as being less harmful and more controllable. An experiment by Friedman et al. (1985) supports this interpretation: giving people the opportunity to ascribe uncomfortable sensation to a non-damaging process increased pain tolerance.

Thus, two attributions have positive effects on pain tolerance: (1) a stable, internal attribution of pain tolerance or pain relief to high levels of ability in coping with pain; and (2) external attribution of sensations to a non-harmful and controllable cause. Interestingly, the first attribution may also suggest to the individual that the cause of the pain is not actually harmful, since something can be done about the pain experience by means of coping strategies ("if the cause is truly harmful, then nothing can be done about it").

\section{Conclusions}

There are several successful coping strategies that can be employed to decrease suffering and to increase pain tolerance and endurance. It is most likely that powerful distraction from pain awareness is the essential factor in these strategies. In order to be effective, subjects have to believe that the strategy helps and that they actually have the ability to use it at will during the painful experience. Without these beliefs, the positive effects disappear. Thus, perceiving control over application as well as perceiving controllable effects of coping strategies are essential. Perceiving control over pain through the use of coping techniques can be promoted by:

1. choice of technique;

2. influence on the timing of elements of the technique;

3. contingent reinforcement of the use of the technique, either through pain relief or other reinforcers;

4. attribution of pain coping to stable, internal sources.

In addition, attribution of pain sensations to non harmful, controllable sources also promotes pain tolerance.

\section{Acute clinical pain}

Various authors have stressed the importance of perceived control with respect to acute pain and its treatment (Turk et al., 1983; Chapman \& Turner, 1986; Weisenberg, 1987). In the following, we will review empirical studies on the relationship between perceived control and acute clinical pain. It is important to make a distinction between various aspects of the acute pain situation: the subjective experience of pain, the subjective emotional experience (worry, anxiety), physiological arousal and behaviours like pain endur- 
ance and medication use. There are two main areas in which research has been conducted: dental pain and pain during childbirth.

\section{Dental pain}

A number of correlational studies have examined the ways in which patients deal with the stress and pain during dental treatment. From this research, which has mainly focused on dental anxiety - and not on dental pain - it can be concluded that there is clear evidence of a relationship between anxiety or the stress experienced during dental treatment and the disproportional expectation of pain and other negative, catastrophizing thoughts about the treatment. The more that patients experience a loss of control over their negative thoughts, as well as over other symptoms, the more anxiety they experience during treatment. There also seems to be a relationship between the degree of which the treatment is experienced as an event outside personal control and aversiveness. Strong support for this conclusion comes from research by Chaves and Brown (1987), who found that $44 \%$ of the patients employed cognitive strategies to minimize pain and stress, while $37 \%$ catastrophized, e.g. exaggerated the negative aspects of the experience. Catastrophizing was related to experiencing more stress during treatment but not to higher levels of reported pain. In line with these findings is a study by Prins (1985), who interviewed 40 children referred for dental treatment to a specialized clinic because of their high anxiety. Only half of the children were actually anxious, and for most of them dental anxiety was based on fear of pain. Prins conchuded that "for most children a visit to the dentist is one undifferentiated aversive event about which not much can be done. Their behaviour is mainly controlled by external contingencies and to a lesser extent by their own plans or rules ${ }^{\text {"1 }}$ (p.650). There was a clear relationship between the child's sense of self-control over his or her fear sensations and the degree of anxiety. Highly anxious children were preoccupied with external, aversive stimuli, with the threat of pain and with escape fantasies. Unfortunately, the study did not report relationships with experienced pain. Similar findings were reported by Kent (1987).

Besides these correlational studies, there are several experimental studies that have investigated the effect of perceived control manipulations on pain as well as on anxiety. In this setting, perceived control is usually experimentally manipulated by giving a group of patients a standardized way to signal the dentist when they want the current activity (like drilling) to stop. This group is compared with patients treated in the usual way.

In general, it seems that giving the patients a way to control the pain-causing activity decreases the experienced discomfort and subjective pain. The objective (behavioural) pain and fear measurements, however, are less clearly linked to the presence of perceived control. This also applies to psychophysiological reactions, and to subjective fear.

Illustrative for these findings is Corah's work. In 1973 he reported an unpublished study be Kruger. Paedodontic patients were given a two-stage signalling device to inform the dentist when they were experiencing discomfort. Thus, patients with this device may have experienced some controll over the dentist's activities by informing him about the level of discomfort. Behaviour ratings indicated that patients with the signalling device were more cooperative and were not as restless as the control patients. Subjective experiences were not reported in this experiment.

In a later study, Corah (1973) heightened controllability in a group of young (6-11 years) dental patients. Here, the device had two buttons; the first to inform the dentist with a green light that the procedure was bothering the child, but not at such a level that he or she wanted the dentist to stop. The second button turned on a red light and a buzzer, upon which the dentist stopped until the child felt more comfortable. The contrast group 
received the usual treatment. In the experimental group, only half of the patients actually used the control device. The experimental group showed fewer spontaneous SCR fluctuations during high-stress procedures (injections of analgesia, high-speed drilling) but slightly more during low-stress procedures. Behavioural ratings of patients' anxiety and cooperativeness were not significant between groups, nor were head and body movements. Unfortunately, no subjective measures of anxiety and pain were taken. Perhaps because of the disappointing findings Corah et al. (1978) tried to replicate this study in a group of adult dental patients, with even more contradicting results: the perceived control group now displayed more spontaneous SCR fluctuations during high-stress procedures. Again, dentists' ratings of anxiety yielded no significant results.

Finally, Corah et al. (1979) compared three experimental groups (relaxation by means of instruction via headphones, perceived control by means of the signalling device, and active distraction by means of playing a video game) with normal treatment. Subjective anxiety was significantly lower in the relaxation and distraction groups, but the perceived control condition did not differ significantly from normal treatment. Patients expressed a clear preference for active distraction, but not for other conditions. Physiologic responses did not show any superionity of perceived control. Unfortunately, again no ratings of pain intensity-pain discomfort were taken. These were, however, included in the studies of Thrash et al. (1982) and Wardle (1983). Thrash et al. used the signalling device discussed above. Dental patients were asked to indicate their level of discomfort continuously by pressing one of three buttons, connected to green, yellow and red light. In one condition (actual feedback, the proper perceived control condition) the dentist stopped when the red light was on, and patients knew that the dentist would stop in the event of a red light. In a second condition (belief in feedback), subjects were shown the device, and were led to believe that the dentist could see the lights. They were not, however told that the dentist would react to their feedback; the lights were actually disconnected, so the dentist could not react. In a third condition (no feedback), subjects were asked to monitor their level of discomfort by the device, but there were no lights so they had no feedback opportunities. Results show that the actual feedback group experienced less discomfort and less pain during injection and also used the red button significantly less frequently than the nofeedback group. The group who believed (at first) in feedback fell between the proper perceived-control group and the no-feedback group. Thus, strong evidence was found for the positive effect of perceived control on experienced pain and discomfort.

Finally, in accordance with Corah et al. (1979), Wardle contrasted three experimental conditions. Dental patients were given either distraction (pictures on the ceiling), detailed information about procedures and the sensations they could expect, perceived control (the patients were asked to raise an arm if they wanted a pause) or none of these. Procedural/ sensations information and perceived control were equally effective on subjective pain. However, only information reduced subjective anxiety significantly. Dentist's ratings of pain and anxiety gave no significant results. The different effects of control and information on anxiety are intriguing. It must be stressed that the control that patients could exercise was not very powerful in this Wardle study (compared to the red lights in the dentist's line of vision or the sound of a buzzer) and patients may have been anxious whether the dentist would see the signal and react fast enough.

\section{Childbirth and perceived control}

Childbirth is a very important experience for many women and men. In contrast with medical treatments, the pain stimulus is not external, and a method to acquire direct control over the presence of the stimulus is not available. Nevertheless, many women and 
men take classes to prepare themselves for the event. Various methods are described for childbirth preparation and many of these attempt to assist women to have relatively painless childbirth (see Mulcahy \& Janz, 1973). Most methods give information about the processes of childbirth and give training in timing, relaxation, breathing and pain-control techniques. Thus, various theoretical ideas on pain control can be found in these methods: breaking the (supposed) fear-tension-pain cycle by relaxation techniques; pairing a breathing response with uterine contractions to replace the experience of pain; knowledge about processes and sensations that might reduce uncertainty; concentration on other aspects than pain (distraction); control of the behaviour in labour, etc: Mulcahy and Janz (1973) state that the integrated "Ema Wright method emphasizes that the individual remains in complete control of the situation by knowledge of what is to occur, utilizing concentration with controlled breathing and active relaxation combined with cognitive and motor activity" (p.423). Numerous studies have shown that learning and applying these techniques results in the use of less medication during childbirth compared with unprepared childbirth (see Manning \& Wright, 1983, for a short review).

In correlational studies, it has been found that the confidence of the woman in the childbirth training (rather than formal characteristics of the training) is related to experienced pain and use of medication (Cogan et al. 1976) and that internals (locus of control) benefit more from these methods than externals (Willmuth $e t$ al. 1978). Interestingly, Felton and Segelman (1978) found that the Lamaze childbirth training changed the women"s belief about personal control.

Brewin and Bradley (1982) investigated the relationship between perceptions of personal and staff control over labour discomfort and over labour duration, and experienced labour pain/discomfort. Women who had followed childbirth preparation classes perceived higher levels of personal as well as staff control. In the group who attended classes, perceived personal control over labour duration was associated with less pain/discomfort experienced during childbirth. This relationship was not found in the group of non-attenders, in which perceived control by the staff over labour discomfort was related to reduce labour pain/discomfort. This study indicates that perceptions of internal controll as well as of control by others can have pain-reducing effects. Manning and Wright (1983) investigated the relationship of expectancy for abillity to control pain without medication during the early stages of labour with actual medication use and medication-free labour time. All women had followed childbirth classes. The belief in the capacity to control the pain correlated with medication use $(r=-0.47)$ and the percentage of time in labour without medication $(r=0.42)$. The general belief that the training would make it possible to have medication-free childbirth was an almost equally strong predictor.

Thus, childbirth training methods seem to result in higher levels of internal control with regard to pain and the process and duration of labour. The level of perceived control varies, however, and the strength of the belief seems to be related to medication use, medication-free labour time, and to reports of pain and discomfort. In addition to these effects of personal control, perceiving control by professional staff over childbirth discomfort seems to be related to reduction in experienced pain.

\section{Conclusions}

Perceived control seems to be an important issue with respect to acute pain. The notion of control is, however, used in very different ways: controllability of various pain symptoms, controllability of the pain stimulus, or controllability of the internal process that causes the pain. Symptoms may be distinguished in cognitive (catastrophizing, attention) physiological (breathing, heart rate, sweating, muscle tension, etc.), emotional (anxiety, 
worry, distress) and behaviour (endurance; medication use, crying, yelling, moving, etc.). Training in methods for limiting these symptoms leads to higher levels of perceived control over symptoms, and this probably increases pain tolerance, reduces medication use, and may also blunt the pain experienced. Giving the subject control over the pain stimulus seems to have clear positive effects on pain and discomfort. However, the effect of this type of control on anxiety and physiologic responses is less clear. Probably, the effect depends on various aspects of the control response and how they are perceived: the difficulty of the response, belief in the power of the response and belief in the rapidity of beneficial effects from the response. For instance, dental patients who are told that the dentist will stop drilling when they raise their arm, may be anxious about whether the dentist will see the arm and will stop promptly.

Research on dental pain indicates that the effects of perceived control over the dentist's behaviour and the effects of procedure/sensation information are comparable. The methods have similarities in that: (1) they decrease the uncertainty about possible future worsening of pain or damage; and (2) they increase confidence that the experience can be endured without fatal damage (since controllability increases self-confidence, while information increases confidence in the professional helper). In sum, perceived control in the acutepain situation decreases the intensity of pain, aversiveness and discomfort by decreasing uncertainty.

\section{Chronic pain}

The potential for developing learned-helplessness (cf. Abramson et al., 1978) is strong arnong chronic-pain patients. This results from their repeated experiences of failing to attain control over pain either by personal action or by medical treatment. In this section, research into the perception of control in chronic pain is first described. Then several aspects of learned helplessness shown by chronic-pain patients, namely generalized perceptions of uncontrollability, lowered self-esteem and depression, decreased ability and motivation to learn, are outlined. Finally, the relationship between perceived control and the treatment of chronic pain will be discussed.

\section{Chronic pain and perceived control over pain}

The present authors asked 22 chronic low back pain patients to rate two types of perceived control: (1) the ability to decrease pain when it is present; and (2) the ability to prevent back pain. Patients perceived relatively low levels of control on both aspects. The ability to prevent back pain appears to be the most important dimension of controllability: it correlates negatively with back pain intensity $(r=-.54, p<0.01)$ and with negative evaluation of having CLBP $(r=-.50, p<0.01)$. Lack of perceived control over pain appears to be an important characteristic of those chronic-pain patients who experience pain disproportionate to organic causes (Reesor \& Craig, 1987). These ("nonorganic") patients felt more ineffective and overwhelmed in their attempts to cope with pain than did organic-pain patients. Interestingly, during an experimental pain induction the former group reported few cognitions reflecting perceived control and manifested more catastrophizing. Thus, these patients showed a generalized tendency to react helplessly to pain.

The perception of uncontrollability of pain can aggravate beliefs that one is disabled and incapable of work and other activities. Arthritic-pain patients who believe that they can exercise little influence over how much their illness affects them lead less-active lives and experience more pain than others (Shoor and Holman as cited by Bandura, 1986). Disability beliefs are associated with more severe suffering in chronic-pain patients (Dol- 
ce, 1987; Feuerstein et al., 1987; Reesor \& Craig, 1987). Sampling the daily experiences of patients with chronic low back pain, Amtz et al. (1988a) found that these patients perceive themselves as helpless, disabled and weak. Avoidance of activities that are (mostly falsely) believed to increase pain is an important characteristic of chronic-pain patients (Philips, 1987). Avoidance has been found to be strongly correlated with beliefs in the lack of ability to self-control pain $(r=-.62$; Philips, 1987$)$.

It is clear that chronic pain is associated with perceptions that pain is largely uncontrollable. The extent of suffering, disability beliefs and passive avoidance are all associated with lack of perceived control. The avoidance behaviour of chronic-pain patients is more reminiscent of the passivity of depressives than the avoidance behaviour of phobies. This similarity between chronic pain and depression is in line with learned helplessness theory. The learned helplessness of chronic-pain patients is characterized by several factors, which will be discussed one by one.

\section{Generalized perception of uncontrollability}

Chronic-pain patients appear to be characterized by a generalized tendency to perceive their activities and their lives as uncontrollable by themselves as well as by others. In their view chance is an important causal agent.

Empirical evidence for generalized perceptions of uncontrollability comes from Srivastava (1983), McGreary and Turner (1984) and Skevington (1979, 1983a): chronic-pain patients seem to have external loci of control and high beliefs in chance. There may be an alteration from personal helplessness (attribution of uncontrollability to oneself) in early phases of treatment to universal helplessness (nobody has control) when chronicity progresses (Skevington, 1983b). The generalized lack of control is apparent in a recent study of the daily experiences of chronic low back pain patients. Compared with normals, these patients report less control over ordinary events and activities during the day (Arntz et al., 1988a). In addition, chronic-pain patients experience high levels of environmental stress, like low work satisfaction, no influence over work characteristics, divorce, death of partner, accidents, etc. (Feuerstein et al., 1987), that are partly objectively uncontrollable, and partly can be perceived as uncontrollable. Prospective research is needed to establish whether environmental stress that is perceived as uncontrollable is a risk factor in the transition from acute to chronic pain. In sum, there is a relationship between chronic pain and generalized perceptions of no control, but it is not clear whether this is a causal factor or a consequence of chronic pain.

\section{Self-esteem and depression}

It is frequently reported that chronic-pain patients have lowered self-esteem (Schmidt \& Amtz, 1987) although this is not always marked (Skevington, 1979; Arntz et al., 1991d). The moderately lowered self-esteem is in agreement with the external-chance attributions that chronic-pain patients make: they do not blame themselves for their pain.

Depressive symptoms appear to be more profound. Depression, as well as anxiety, is related to perceiving that pain coping strategies are ineffective in patients with chronic low back pain (Rosenstiel \& Keefe, 1983). Hopelessness and depression may well be both a consequence and a cause of the continuation of perceived uncontrollability.

\section{Decreased ability and motivation to learn}

Learned helplessness theory predicts that chronic-pain patients will show decreased ability and motivation to learn to control their pain and to learn to increase physical functioning. Research on physical performance does indeed indicate that chronic low back 
pain patients do not show learning and are less influenced by external information about their performance (Schmidt, 1985). These patients have difficulties in learning to alter activities in circumstances that cause low levels of back pain, and may therefore overexert until pain becomes very intense (Nalibof et al., 1981). A related characteristic of chronic-pain patients is the combination of increased pain threshold and decreased pain tolerance (Schmidt \& Arntz, 1987). It is as yet unclear whether this characteristic has always been there or is caused by chronic pain. There are clinical indications that chronicpain patients had increased pain thresholds before chronicity, and had a tendency to overexert without feeling physical warning signalls. Probably, these patients have formerly experienced high levels of control over physical functioning. In any case, increased pain threshold and decreased pain tolerance may be important factors in maintaining the perceived uncontrollability of pain: at lower levels of noxious stimulation nothing is felt, so that patients cannot learn pain prevention responses; and very quickly the pain is experienced as intolerable and overwhelming, making the patient a helpless victim.

\section{Perceived control and the treatment of chronic pain}

As appears from the above that the behaviour of chronic-pain patients can partially be explained using the learned helplessness concept. This implies that controllability will also play an important role in treatment. In fact, it has been reported that chronic-pain patients with severe learned helplessness deficits show less improvement with psychological or medical treatment (Thomas \& Lyttle, 1980; Chapman \& Brena, 1982). When improvement takes place, it appears to be especially large in perceived control over pain and in related areas (Philips, 1987; Flor et al., 1983). Before further elaboration, the special situation of the chronic-pain patient who appeals for treatment should be emphasized.

First, there are clinical indications that chronic-pain patients perceive that they formerly had high levels of control over physical functioning. Experiencing severe acute pain and the associated physical limitations may have resulted in a major loss of control for these subjects. From laboratory research, it is known that loss of control is more stressful than never having had control at all (Overmier et al., 1980; Staub et al., 1971).

Second, chronic-pain patients are known to strive for total pain relief, viewing pain as a primarily medical problem that should be medically treated. As long as chronic-pain patients try to acquire this kind of control, they are doomed to experience repeated failures, continuing their helplessness. The kind of control that can be acquired first requires acceptance by the patient of having pain. One cannot learn pain coping or prevention strategies without accepting that one has pain. There are indications that subjects who do not accept unchangeable aversive facts suffer more, and adapt less (Silver et al., 1982; Rothbaum et al., 1982). Thus, it appears to be necessary to refrain from trying to acquire total control of what cannot be controlled in order to perceive control of what can be controlled.

The relationship between chronic pain, low perceived control and the other factors discussed above can be integrated in a hypothetical model (figure 2). The core of the model is the vicious circle between low perceived control and the experiences of pain and depression.

Of the many different chronic pain treatments, such as operant reinforcement of nonpain behaviour, relaxation training, cognitive-behavioural therapy, EMG biofeedback, exposure to pain-increasing stimuli, etc., one is especially suited to illustrate the role of perceived control in treatment: this is EMG biofeedback training for tension headache and chronic low back pain. 
Although the rationale seems clear (muscle tension leads to pain), no stable relationships between EMG decreases and decreased subjective pain have been found (see Turner \& Chapman, 1982; for a review). However, EMG biofeedback has been found to be superior to placebo treatment or no treatment.

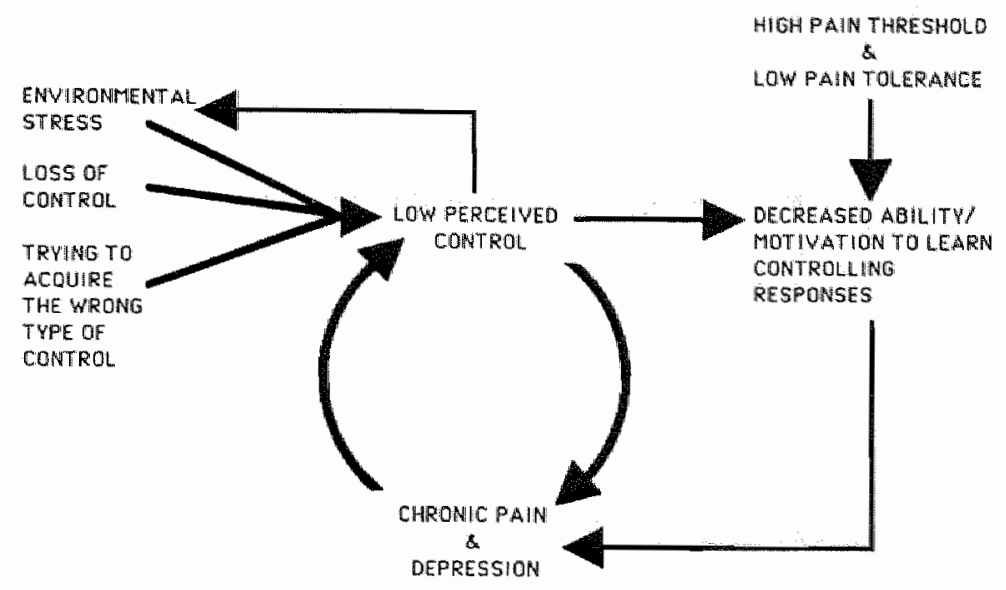

Figure 2. A hypothetical model of the relationship between chronic pain and low perceived control

Thus there seem to be other factors that result in pain relief. Nouwen and Solinger (1979) found that those chronic low back pain patients who experienced successful EMG decreases coupled with reductions in experienced pain showed stable improvement at follow-up. Those patients who were less successful did not show improvement. However, at follow-up there was no relationship between EMG and improvement in back pain. This paradox led Nouwen and Solinger to hypothesize that the successful patients perceived control over tension and pain, and that this control mediated final success. Holroyd $e t$ al. (1984) tested this hypothesis in a fascinating experiment. In a two by two design, headache patients received either audio feedback (lower tones suggesting tension decrease) contingent on decreased EMG or on increased EMG. The second factor was the degree of success (high v. low), and this was experimentally manipullated by means of bogus graphic display of EMG activity during and across sessions. High success feedback, regardless of direction of actual EMG, produced significantly greater improvement in headache complaints and medication use. Again, improvement was not related to EMG. Interestingly, high-success feedback also caused changes in locus of control and in self-efficacy. Bush $e t$ al. (1985) report similar findings, in that paraspinal EMG feedback and bogus feedback produced equal improvement in chronic low back pain patients. Another study (Biederman et al., 1987) manipulated the size of the feedback scale on the apparatus (but in all conditions feedback correlated with actual EMG decrease) and did not find significant effects. However, in this study, the number of patients was very small and the success manipulation relatively weak.

In summary: patients' helplessness and perceived uncontrollability are central themes in psychological treatment of chronic pain. They are areas in which mucht progress in treatment can be achieved. It is very probable that improvement during and after treatment is mediated by perceived control over pain and other problems. The important factor appears not to be the actual technique that is applied, but whether the patient perceives it as helpful and applicable. 


\section{Conclusions}

It appears that chronic-pain patients show characteristics of leamed helplessness as a result of experiencing chronic uncontrollable pain and stress. They perceive low control over pain and life and are avoidant in their behaviour. Thus, the relationship between low perceived control, learned helplessness and psychological suffering is apparent. It may be hypothesized that these patients suffer especially from failing to acquire total control (and complete pain relief). Instead of accepting pain and trying to acquire some control by means of coping strategies, they become helpless and think that nobody can do anything. On the other hand, psychological treatments can be effective in reducing pain and psychological suffering. Letting the patient perceive control over pain seems to be an essential mediator of treatment success.

\section{WHY DOES PERCEIVED CONTROL REDUCE PAN?}

Previous reviews of the effects of perceived control on the experience of aversive events (not restricted to pain) have come to different conclusions. Averill (1973) concluded that controllability does not reliably decrease the impact of aversive events. He states that effects of controllability depend upon the meaning of the controlling response for the subject, but theoretical predictions about the determinants of meaning and implications of different meanings remain vague. Miller (1979) concluded that controllable events may hurt less. She discussed ten theories that purported to explain the positive effects of control and concluded that seven are based on circular reasoning (e.g. explaining why control reduced physiological anticipatory arousal by stating that controllable stress is less arousing, without explaining why this is). However, the three remaining theories cannot explain all findings, most importantly the effect of controllability above predictability. Miller proposed a new theory (the "minimax" hypothesis), which explains positive effects of control as follows. In controllability conditions, the subject can rely on a stable, reliable source (his or her own response) than can modify the aversive event. Therefore, future danger can reliably be kept below an acceptable maximum level. When you have no control, there is no guarantee that future danger will be restricted. The hypothesis explains why subjects prefer control and show less anticipatory arousal. However, according to Miller the minimax hypothesis cannot explain the decreased impact of aversive events.

Finally, Thompson (1981) concludes in her review that controllability does not influence experienced aversiveness. Thompson states that the meaning of the aversive event is the important issue, but remains (like Averill) rather vague about implications of this assertion.

In this chapter, the focus was on pain, and other types of aversive events were excluded. Laboratory as well as clinical studies were discussed. There appears to be ample evidence that controllability can reduce the experience of pain and can promote tolerance and endurance.

The question will now be addressed of why perceived control may have these effects. With the exception of one theory, no theories that are circular according to Miller will be discussed. In addition, no theories will be discussed that cannot explain the superior effects of controllability above predictability. Five explanations can be put forward. 
Anxiety increases the experience of pain and controllability reduces anxiety.

Although a rather popular explanation, there are two problems with it. First, is it true that anxiety (or the associated physiological arousal) increases pain? Second, does control always reduce anxiety in cases where it reduces pain?

\section{Does anxiety increase pain?}

The frequently observed correlation between acute pain and anxiety (Chapman \& Turner, 1986) should not be confused with a causal effect of anxiety on pain. Chapman and Turner (1986) state that anxiety has physiological effects that increase the pain response: increased sympathetic activity and the release of epinephrine at the sympathetic terminals may sensitize or directly activate nociceptors; increased muscle tension, especially near the site of the wound, may cause additional pain. A psychological explanation might be that intense anxiety causes loss of control over thinking (Kent, 1987), which deprives the person of one of the most powerful coping strategies, - distraction. Bolles and Fanselow (1980), however, have proposed a controversial theory, which implies that fear and pain are mutually exclusive states. Fear is assumed to decrease the experienced pain and to activate the organism (flight-fight response), whereas pain is assumed to deactivate the organism, promoting healing. Their theory is based on findings in animal studies and the applicability to human pain is not clear yet.

In humans, research findings give conflicting results. Kleinknecht and Bernstein (1978), Dougher (1979), Weisenberg et al. (1985), Ahles et al. (1987) and Malow et al. (1987) report positive correlations between state anxiety and subjective (externally caused or internal spontaneous) pain. Klepac et al. (1982) found that dental patients who avoided treatment showed reduced pain tolerance, but that pain threshold and subjective pain were not different from those of dental fearless patients. Two studies have investigated the effect of experimentally induced anxiety on pain. In the first (Malow, 1981), anxiety induction was related to a greater tendency to report pain. In the second (Dougher et al., 1987) only anxiety that had as its focus the pain stimulus had a pain-increasing effect.

In contrast to these positive findings, many studies found no significant relationships (Arntz et al., 1990a; Bowers, 1968; Chaves \& Brown, 1987; Klepac, et al., 1980), or even negative relationships, as in a study of Kent (1984) who found that highly anxious dental patients experienced less pain during treatment than low-anxious patients.

Also in contradiction with the hypothesis, reducing anxiety and physiological arousal does not necessarily have strong pain-relieving effects, as the research on relaxation shows. The effects of relaxation and biofeedback seem not to be mediated by decreased physiological arousal (see above). Positive effects are probably caused by distraction from pain by actively involving the subject in various exercises.

Thus, on the one hand it is generally accepted that, in human beings, acute pain can be accompanied by subjective anxiety and physiological arousal. On the other hand, it is not clear whether anxiety and physiological arousal increase the experience of pain. It might by hypothesized, however, that when the focus of anxiety is on pain, the pain experience is more intense and tolerance is lower. The critical factor then seems to be attention, and not anxiety. A frightened wounded soldier who runs for life will probably experience no pain when he tries to escape the battle ground.

\section{Does control always reduce anxiety in cases where it reduces pain?}

Laboratory studies indicate that this is not always the case (table 1). As has been discussed, research on coping strategies shows that when subjective pain and pain tolerance 
are positively affected, anxiety and physiological arousal are not always reduced. Similar1y, anxiety during dental treatment does not seem to be reliably influenced by giving the subject control over the dentist's behaviour, whereas pain is affected. In short, perceiving control over pain or its cause can lead to pain relief and enhanced pain tolerance without any associated decrease in physiological arousal or subjective anxiety.

Since both parts of this anxiety-reduction hypothesis appear to be questionable, it seems unlikely that positive effects of perceiving control are mediated by decreased anxiety or decreased physiologicall arousal.

\section{Perceiving control promotes endogenous opioid release}

This explanation presumes that control promotes the release of natural analgesic opioids that reduce the experience of pain. Several arguments can be made against this explanation.

First, perceiving no control over stress appears to be related to endogenous opioid release, in contrast to perceiving control, as basic animal research has shown (Maier et al., 1983; Watkins and Mayer, 1982). It has been argued that the same processes that lead to learned helplessness deficits trigger endogenous opioid release (Maier et al., 1983). Moreover, chronic-pain patients who suffer disproportional to somatic causes display heightened opioid levels as do depressive patients (Terenius, 1985; Almay et al., 1978), probably as a result of experiencing chronic uncontrollable pain. Thus, endogenous opioid release seems to be related to uncontrollable pain, and not to perceiving control over pain.

Second, since the organism develops tolerance for endogenous opioids (Terman et al., 1984), the pain-decreasing effects of control would be only temporary if they were based on endogenous opioids. Treatment studies of chronic pain patients have, however, shown that increased control can have long-lasting positive effects.

Third, it seems unlikely that the chronic-pain patients who suffer emotionally and behaviourally most from pain, and already have very high levels of endogenous opioids can profit from still higher levels. Perceiving control can, however, help those patients. Most likely perceiving control is related to a decrease in opioid levels caused by a diminution of perceived uncontrollability.

Thus, it is unlikely that the effects of control are mediated by increased endogenous opioid release. However, other endlogenous analgesic mechanisms may play a role, although research has not yet demonstrated such effects.

\section{Controllability changes the meaning of pain or the meaning of the cause of the pain}

The experience of pain depends strongly on cognitive-emotional factors. On the basis of the meaning of the pain or its inferred cause, pain can be felt in very different degrees (Beecher, 1956). We hypothesize that the inferred harmfulness of the pain or its cause is the central issue. It can be argued that control over pain or its cause changes the meaning of the pain and hence the way it is experienced. Pain that cannot be controlled may be appraised as caused by a harmful process. Pain that can be controlled may be appraised as non-harmful and hence will have a different significance for the subject. Thus, the subject may reason that if the pain (or cause) is truly harmful, nothing can be done about it. And conversely, if something can be done to decrease it (leading to perceived control), that it means that the pain (and its cause) is not truly harmful. Some evidence for this explanation has been discussed. However, its role in the effects of controllability deserves fuller investigation. It may apply especially to clinical pain or possibly harmful laboratory pain, 
but it cannot explain all laboratory findings on the effects of behavioural control over an external pain stimulus, such as control over the duration of the stimulation.

Perceived control is a positive experience per se which can compensate negative experiences

Several authors have proposed that acquiring and exercising control is a positive experience per se, and that having no control or losing control is a negative experience. Averill (1973) argues that controllability has acquired these qualities in evolution (because of its survival value). Psychoanalytic authors have stressed its value for the development of the child (Fisher, 1986). Control has also been seen as a primary pleasure (Fisher, 1986) or an unconditioned reinforcer (Miller, 1979). What is not implied in this explanation is the reinforcement the subject experiences when he or she actually stops or decreases pain. What is meant is that knowing that the pain can be controlled or perceiving control without actually exercising it has a positive effect by itself. Miller argues that this explanation is a circular one. However, there seems to be no reason why it cannot be tested empirically.

\section{Perceiving control allows people to distract from pain}

The core of this explanation is the simple fact that pain cannot be felt when it is not in awareness. Perceiving control over pain gives the subject certainty that the pain (or its cause) will not exceed unbearable or unacceptable limits. Hence, subjects do not have to attend continuousty to internal signals of impending danger and can distract themselves. Attention to pain increases the pain experience; distraction decreases it. This explanation is actually an extension of Miller's minimax hypothesis. It is a parsimonious explanation and can be empirically tested. Like Miller's minimax hypothesis, it can account for the fact that under certain circumstances subjects choose to give others control (e.g. to the professional doctor), when these others are perceived as more reliable sources of control.

It can also explain why subjects are willing to tolerate or endure more pain when having control (because they have higher certainty that they can end the pain than when having no control) as well as why the experience of pain can be less intense and less emotional under these circumstances. It may account for the observation that the effects of perceived control over the dentist's behaviour and the effects of procedural and sensation information are comparable: the interventions are similar (1) in that they decrease the uncertainty of the subject about future worsening of pain or damage (control by giving the subject an opportunity to stop the cause of the pain, information by establishing the limits of treatment and its sensory effects); and (2) in that they increase confidence that the experience can be endured without fatal damage (control increases self-confidence, information increases confidence in the professional helper). Thus both procedures probably decrease uncertainty and increase confidence, and these allow the subject to distract themselves.

The last three explanations may in fact all play a role in the effects of controllability. It certainly seems worth while investigating them. For too long have the positive effects of perceived control over pain been taken for granted. 


\section{SECTION 4.2. \\ THE INTERACTION BETWEEN MOOD \\ AND CONTROLLABILITY"}

\section{SUMMARY}

The present study examined the hypothesis that induced mood influences the effects of control. Two possible mechanisms were investigated: (1) mood exerts its influence by influencing perceptions/expectations of control; (2) control exerts its effects by inducing a positive mood which compensates for aversive experiences. In addition, the hypothesis was tested that control leads to increased certainty about the amount of pain to be expected. Forty $S$ s all received 20 painful stimuli, which they tried to stop by pressing a combination of buttons. In a $2 \times 2$ design, induced mood (elation ws depression) was combined with control $v s$ failing to acquire control over pain offset. The results indicated that elated mood induction and control reduced pain impact, compared to depressed mood induction and no-control. Control was related to increased certainty and reduced SCRs, but mood was not. Without control, elated Ss were more persistent in trying to acquire control. There was no evidence that mood influenced subjective pain impact via perceptions of control than depressed $S \mathrm{~s}$. There was more evidence in favor of a model in which mood and control have separate, additive influences on pain.

\section{INTRODUCTION}

It is well known that perceiving control over an aversive stimulus, such as pain, can reduce the aversiveness of the stimulus (Miller, 1979; Mineka \& Henderson, 1985; Arntz \& Schmidt, 1989). The present study aims at clarifying whether mood influences the effects of (un)controllability. It might be theoretically and clinically interesting if elated mood can compensate for the negative effects of uncontrollable aversive events, and if depressed mood decreases the positive effects of control. If mood influences the effects of (un)controllability, the question arises whether mood exercises its influence by influencing perceptions of control. This model can be schematized as follows:

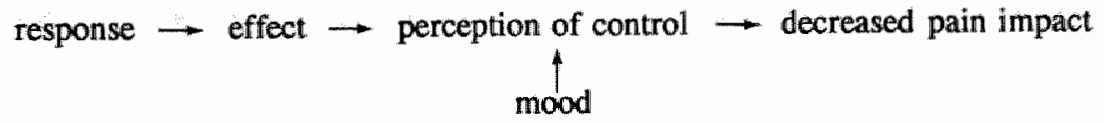

It is also possible that exercising control by itself induces a positive mood, that compensates for negative events. In other words, controllability and mood inducing factors might interact on a common dimension of mood, and exert their influences via this dimension:

mood inducing stimuli

exercising control $\rightarrow$ positive mood $\rightarrow$ decreased pain impact.

'Submitted for publication by Arntz, A., Gransier, S., de Jong, P., Oosterlaan, J., Gubbels, I. \& Jansen, $\mathbf{A}$. 
In addition to studying the influence of mood on effects of controllability, this study aims at testing a hypothesis pertaining to a possible mood independent mechanism of control. In short, the hypothesis is that when the $S$ has control, he/she is highly certain that the aversive stimulus will remain below unbearable limits, and he/she can therefore distract hisher attention from the stimulus, which results in a smaller pain impact:

perceiving control $\rightarrow$ increased certainty $\rightarrow$ distraction $\rightarrow$ decreased pain impact.

These three possible mechanisms, of which the first two are mood dependent and the third is mood independent, will be discussed in more detail below.

The first hypothetical mechanism pertains to perceptions of control. It is generally assumed that the $S$ should first perceive that he or she is in control, before being in control can have any positive effects. There are indications that mood is an important factor in this perception process. It has been shown that induced mood influences estimates of past success; depressed $S$ s make lower estimates than elated $S$ s (Teasdale \& Spencer, 1984). In addition, induced depressed mood appears to be related to giving up a response more quickly if it is no longer reinforced (Gouaux \& Gouaux, 1971). In a series of experiments; Alloy and co-workers (Alloy \& Abramson, 1979; Alloy, Abramson \& Viscusi, 1981; see also Rhodewalt, Strube \& Wysocki, 1988) demonstrated that elated mood is related to overestimation of control over neutral or positive outcomes (such as winning money). Depressed mood, on the other hand, appears to be related to a realistic estimation of control. Thus, mood seems to influence the perception of control and related issues, such as expectations of success and persistence behavior.

Whereas there appears to be clear evidence for the influence of mood on perception of control over neutral and positive experiences, it is still unclear whether the effects of control over an aversive stimulus are also influenced by mood. In other words, the question arises whether mood influences the effects of having control, as well as the effects of failing to acquire control, on the impact of aversive experiences by influencing perceptions of control. Alloy et al. asked for ratings of perceived control after the task, which gives rise to the question whether perceptions of control during the task are influenced by mood. In the present experiment indications of perceived control were obtained during the task.

Another hypothetical mechanism by which mood and control can interact might be related to mood itself. One possible explanation for the favorable effects of controll is that having control is a positive experience per se, which can compensate for negative experiences (Arntz \& Schmidt, 1989). Several writers have theorized that having control is a positive experience in itself and that not having control or losing control is a negative experience. Averill (1973) hypothesized that control has acquired these qualities in evolution because of its survival walue. In developmental theories, acquiring and exercising control is often assumed to give a child pleasure, despite the seemingly meaningless play activity (e.g., Fisher, 1986). There is also evidence that acquiring and exercising control is related to self-esteem and a pleasurable feeling of freedom (cf. Abramson \& Alloy, 1980; Brewin, 1989), whereas failing to acquire control or losing control may be a threat to, or may even mean a loss of self-esteem and freedom. It may be true, then, that control has an intrinsically positive effect which compensates negative effects of aversive experiences. It is worth to investigate whether this hypothesized effect of control is apparent from emotional indices, and whether effects of control interact with the effects of mood induction on the impact of aversive stimuli.

If control decreases the impact of negative experiences by influencing mood, two 
implications follow: (1) positive mood should have a pain decreasing effect in itself (there are indeed indications that there is such an effect, e.g. Martin et al, 1988); and (2) having control should improve mood, whereas failing to acquire control should impair mood.

A quite different explanation of the effects of control is that perceiving control over essential aspects of the aversive stimulus allows people to distract their attention from the painful experience (cf. Amtz \& Schmidt, 1989). There is considerable evidence that attention influences the impact of aversive experiences; attention to the stimulus increases the impact, distraction decreases it. It was reasoned that this explanation is an extension of Miller's minimax hypothesis (Miller, 1979), in that it is assumed that the certainty which Ss have when they are in control, that the aversive stimulus will not exceed unbearable or unacceptable limits, allows them to distract their attention. On the other hand, it is assumed that, when they are not in control, Ss pay more attention to the stimulus, mindful of signals of impending danger, because they are uncertain about the final consequences. If this is true, it may be hypothesized that $S$ s are more certain about the aversiveness they expect to experience under conditions of control than under conditions of no-control. This hypothesis suggests that controllability effects may not be mediated by mood.

In order to investigate the influence of mood on the effects of control over pain, mood was induced by having the $S \mathrm{~s}$ listen to music. This is one of the most powerful ways to induce mood (Clark, 1983). We tried to influence the Ss' mood during the whole experiment by playing music continuously. Two moods were induced: depressed mood and elated mood. The $S$ s were instructed to try and find a code, by which they could stop the painful stimulus. If they tried the wrong combination, the stimulus would be stopped by the computer later on. Half of the $S s$ were given control over the end of painful stimulation from the 5th trial onwards. The other half were not given control and therefore experienced repeated failure to execute control. Both groups received an identical amount of painful stimulation. Control over the offset of pain was chosen, because there are clear indications that control that is related to limitation of the aversive stimulus has positive effects, in contrast to other types of control, such as self-administration of the stimulus (Arntz \& Schmidt, 1989). The design offers the possibility to assess the influence of mood on the effects of having control over pain, as well as the influence of mood on the effects of failing to acquire control.

Four hypotheses were formulated about the influence of mood, and one hypothesis about the assumed certainty increasing effect of control.

- First, it was hypothesized that the effects of control over pain are influenced by mood; that is, elated $S$ s who are given control over pain experience less pain than depressed $S$ s who are given control. And similarly, we expected that effects of failing to acquire control would be smaller when the $S$ is in an elated mood than when the $S$ is depressed.

- Second, it was hypothesized that perceptions and expectations of control are influenced by mood: that is, when they are elated, $S s$ have higher perceptions/expectations of control than when they are depressed. We expected these effects in the control condition, as well as in the no control condition.

- Third, we hypothesized that depressed mood is related to giving up trying to find the controlling response in the no control conditions.

- Fourth, it was hypothesized that mood and (not) having control would interact on the affective dimensions of (1) depression - elation, (2) nervousness and (3) anger: having control was expected to be related to good moods, having no control to bad moods.

- Fifth, it was hypothesized that with control, $S$ s expect less pain, or are more certain about the amount of pain to be expected, than without control. 


\section{METHOD}

\section{Subjects}

The study was carried out with 40 normal $S$ s, all women, because there are indications that mood induction is easier with women (e.g., Velten, 1968; Alloy, Abramson \& Viscusi, 1981 , note 1). The mean age was 41 years and $74 \%$ were housewives. Subjects were recruited by means of an advertisement in a local newspaper. They received a small remuneration for participation.

\section{Design}

The design was a 2 ('MOOD'; depressed $v s$ elated mood) $\times 2$ ('CON'; control vs no control) factorial one. There were 20 trials in which the $S$ could try a response. The first 4 trials were uncontrollable for all $S$ s, that is, the responses were not followed by an immediate termination of the pain stimulation. From the fifth trial onwards, $S s$ in the control conditions were given control over all trials.

\section{Materials}

Subjects rated subjective mood before and after the mood induction on three $10 \mathrm{~cm}$. Visual Analogue Scales (VASs), one for depression - elation (downcast - elated), one for nervousness (calm - nervous), and one for anger (not angry - angry). After each triall, eight VASs were rated: (1) experienced pain (not painful at all - extremely painful); (2) perceived control over the offset of the stimulus ("To what extent did you experience a sense of control over the offset of the pain stimulation ?'; no control at all - complete control); (3) depression -elation; (4) nervousness; (5) anger; (6) predicted pain of the next trial (not painful at all - extremely painful); (7) certainty of this prediction (not certain at all - completely certain); (8) expectation of control ('how certain do you feel that you can stop the next stimulus "; not certain at all - completely certain).

\section{Apparatus}

Apparatus was placed in an adjacent room. Skin Conductance Responses (SCR) and Skin Conductance Level (SCL) were measured by a Beckman Skin Conductance Coupler (type 9844), using the method of constant voltage (0.5 volts). The coupler allowed for a maximum sensitivity of 0.05 micromho. Electrodes were attached to the medial phalanges of the second and third fingers of the non-dominant hand with adhesive collars. Respiration was measured by a Beckman Voltage/Pressure/Volume Coupler (type 9853A) via a Hewlet-Packard respiration belt fastened around the S's chest. SCR, SCL and respiration were continuously monitored by a Beckman Polygraph. The painful stimulations were delivered by an electric stimulator, passed for use with patients (Siemens Neuroton 627), on the $S$ s right ankle. The stimulation rose to its maximum level in $2 \mathrm{sec}$, remained at this level for the programmed time, and subsequently decreased to nil in 2 sec. Subjects could try a code by pressing buttons on a panel with five buttons. The panel was connected with a panel in the adjacent experimenters' room, displaying the code by means of bulbs. Feedback was given by means of small bulbs on the panel, a green bulb with the caption 'right' and a red bulb with the caption 'wrong'. There was also a button on the panel with the caption "no code", by which the $S$ could indicate that she did not try a 
code. An intercom allowed the experimenter to signal to the $S$ that she could try a code (by saying 'code'). A buzzer announced shock offset. The music was played via two loudspeakers. A microcomputer (PDP Minc-11) controlled the experiment.

\section{Procedure}

Subjects were randomly allocated to one of the four conditions. In the pretest phase the objective pain stimulus level was individually callibrated to a subjective pain level of approximately 70 on a 0 (not painful at all) to 100 (extremely painful) visual analogue scale. Three moods were assessed by means of visual analogue scales: elation, anger, and nervousness. Half of the $S$ s were subjected to a depressed mood induction by having them listen to 'Russia under the Mongolian yoke' by Prokofiev, played at half speed fo 9 min; whereas the other half were subjected to an elation induction by having them listen to music from Charlie Chaplin films. The $S$ s were not instructed about the kind of mood they were expected to get into, in order to reduce experimental demand effects. They were instructed as follows: 'Music can induce certain moods, for instance music can make you feel sad, anxious, or happy. We would like you to listen to the music and get into the mood that is elicited by the music as much as possible. We understand that it seems a bit. silly to let your feellings go here in the laboratory. But, we ask you to try. Try to get into the mood that the music is eliciting." After mood induction, current mood was assessed again and $S$ s started on the task of finding the response that could stop the pain stimulus during a series of 20 trials. Half of the $S$ s were assigned to the control conditions, the other half to the no control conditions. In the control conditions, the $S$ s were given control from the 5 th trial onwards by stopping the pain stimulus immediately after the combination they tried at the 5th trial. During the whole experiment music was played: Prokofiev and Albinoni's Adagio in the depression conditions, and in the elation conditions various pieces of music, such as film music (Charlie Chaplin, Nina Rota), but also classical music, for instance by Mozart and Johann Strauss.

Figure 1 shows the way in which we manipulated controllability. Trials without control were designed as follows:

- The painful stimulus started unannounced. After a preprogrammed interval $(7,4,8,7,5$, $5,6,8,6,7,4,6,5,7,5,4,4,5,6 \& 6$ sec for trial $1-20$ ), a signal (the instruction 'code" via the intercom) was given that the $S$ could try to stop the stimulus by choosing a code on the panel with 5 buttons.

- Feedback was given by means of a red bulb, with the caption 'wrong'.

- The pain stimulus continued until its programmed offset. The total duration of stimulation varied from 13 to $19 \sec (16,14,18,17,14,13,15,17,15,19,15,16,14,19,16,18,13$, $14,17 \& 18 \mathrm{sec}$ for trial 1-20).

- Two seconds before the end of the stimulus a warning signal was given by means of a buzzer, to control for the effects of predictability of the end of the stimulus.

Trials with control were designed as follows:

- The duration of each trial was identical to those of the no-control conditions.

- After the unannounced start of the painful stimulus, a signal was given that the $S$ could try his/her code. In contrast to the way it was done in the trials without control, this signal was given much later, 2 seconds before the end of the stimulus. The end of the stimulus was accompanied by feedback by means of a green bulb with the caption "right".

There are two important points in this manipulation: first, the length of stimulation was identical in the control and in the no control conditions, as is illustrated in figure 1 . Se- 
cond, since it is known that predictability of essential aspects of the stimulus can decrease the impact of the stimulus, predictability was kept equal in all conditions: the predictability that Ss experienced in the control conditions because they knew that the stimulus stopped after their response, was controlled for by the warning signal in the no control condition. This manipulation guarantees that effects of control cannot be attributed to objective differences in the amount of painful stimulation, or to differences in predictability.

The shocks had a mean duration of $16 \mathrm{sec}$ (range 13-19 sec). In the case of the no control shocks, the signal "code" was given on average $5.75 \mathrm{sec}$ after the onset of the shock (range $4-8 \mathrm{sec}$ ). In the case of the control shocks, this code signal was given $2 \mathrm{sec}$ before the offset of the stimulus. Ss in the control conditions who tried a wrong code (that is, a different code from the one they tried at the 5th trial) received a longer shock duration: 6 seconds longer (to ensure credibility of the manipulation). There were, however, very few instances of such trials. The intertrial intervals varied from 50 to $70 \mathrm{sec}$ (mean $60 \mathrm{sec}$ ). The mean objective shock intensity was $1.9 \mathrm{~mA}$ (s.d. 0.85 ).

\section{CONTROLLABILITY MANIPULATION}

\section{TRIALS WITHOUT CONTROL}
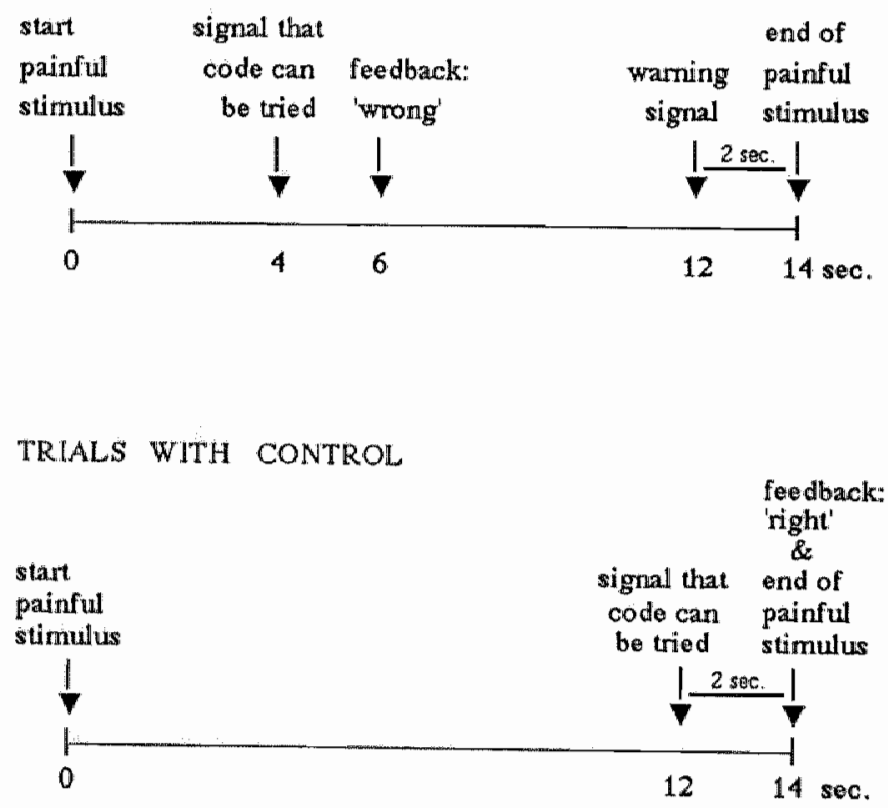

Figure $I$. Illustration of the controllability manipulation. Trials without control had an early signal that a code could be tried. Two sec after this signal, feedback ("wrong') was given. To control for predictability of the offset of the stimulus, a warning signal was given $2 \mathrm{sec}$ before offset. In the case of trials with control, the signal that a code could be tried was given $2 \mathrm{sec}$ before pain offset. At offset, feedback 'right' was given. Note that this design controlled for stimulus duration and predictability of stimulus offset. 
Responses to the 20 trials were analyzed in blocks of 4 trials. Skin conductance impact response was defuned as the largest deflection, compared with the level just before shock onset, starting after shock onset and with a response window of $4 \mathrm{sec}$ (this is before a SCR to the earliest 'code' signal in the no control conditions could take place). SCRs due to respiratory irregularities were replaced by estimated responses (on the basis of interpolation). SCRs were transformed by square root (Levey, 1980). For subjective and physiological responses, ANOVAs were planned for the first block, before the control manipulation had been introduced. The next four blocks would be analyzed by means of MANOVA trend anallysis: the first trend corresponding to the average response was considered as the critical trend, but sometimes the linear trend was also taken into consideration. The SPSSX output yields t-tests which correspond to the hypothesized effects (allowing onetailed testing of the hypotheses). There were two factors: MOOD (depressed vs elated mood induction) and CON (control $v s$ no control). For experienced pain and SCRs, objective shock intensity and subjective pain rated at the calibration were employed as covariates in a stepwise method: if their contribution was $n . s_{.}$, they were deleted from the analysis. The same procedure was used for mood, with pretest mood scores as covariates.

\section{Selection of subjects}

Because of technical problems with the shocker, $6 \mathrm{Ss}$ did not complete the protocol. One $S$ in the control condition forgot her controlling response, and could therefore not exercise control. Two $S$ s showed mood changes opposite to the intended direction (a conservative criterion was employed: all $S$ s showing any mood change in the expected direction, or no change when already very much elated (score $>90$ ) were included). These $8 S$ s were replaced by new $S$ s.

\section{RESULTS}

\section{Mood induction: immediate effects}

There were no differences between groups at the pretest, before the mood induction. Whith the pretest VAS score as covariate, ANOVA indicated a significant effect of the mood induction on the depression-elation VAS $\left(F(1,35)=51.32, p<10^{-3}\right)$. The $\mathrm{CON}$ and CON $\times$ MOOD interaction effects were n.s. $\left(p^{\prime \prime} s>0.50\right)$. As can be seen in figure 2, mood dropped after the depressed mood induction, whereas mood rose after the elated mood induction. The mean between effect of $37 \mathrm{~mm}$. is comparable to the effect reported by Clark \& Teasdale (1985, as cited by Clark, 1983).

The effects of the mood induction on nervousness and anger were less strong: $F(1,34)$ $=7.59, p<0.01$ and $F(1,34)=5.87, p=0.02$ (data from one $S$ were missing; pretest moods served as cowariates). In the ellated conditions, $S$ s became less nerwous and less angry; in the depressed conditions $S$ s became angrier but not more nervous. In conclusion, the mood induction had the clearest effect on the depression-elation dimension. 


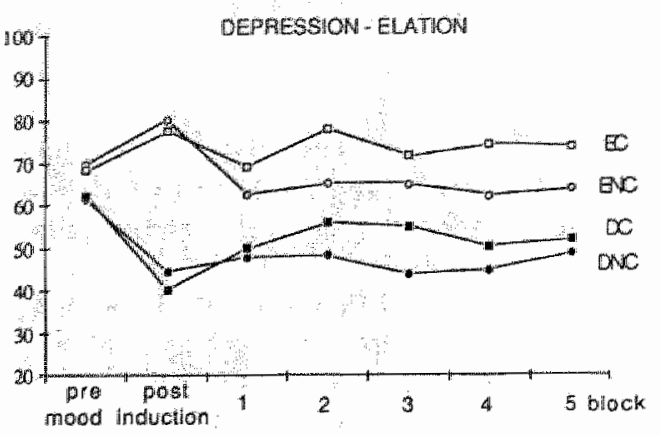

Figure 2. Mood in the four conditions, pre and post mood induction, and averaged scores of blocks of 4 trials. Low scores indicate depressied mood, high scores elated mood. $\mathrm{EC}=$ ellated mood induction/control; $\mathrm{ENC}=$ elated mood induction/no control; $D C=$ depressed mood induction/control; DNC = depressed mood induction/no control. There is a clear effect of mood induction, which lasts the whole experiment. There was a marginally significant $(p=0.10)$ effect of controllability on mood.

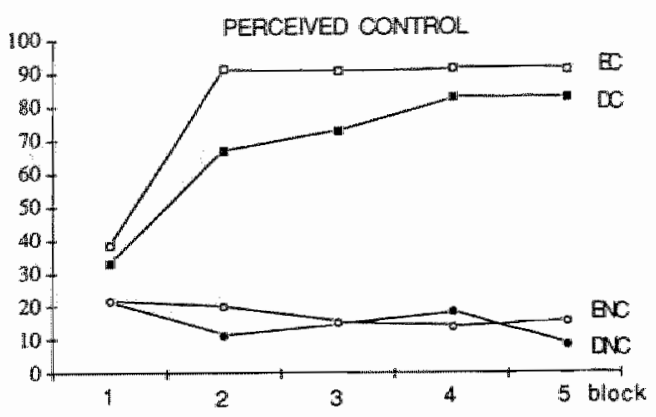

Figure 3. Perceived control ratings, aweraged in blocks of 4 trials. When Ss were given control, perceived control ratings rose sharply. There was a MOOD effect on this rise: depressed Ss showed a slower rise. Without control, no MOOD effect was apparent. EC = elated $\mathrm{mood}$ induction/control; $\mathrm{ENC}=$ elated $\operatorname{mood}$ induction/no control; $D C=$ depressed $\mathrm{mood}$ induction/control; $\mathrm{DNC}=$ depressed mood induction/no control.

\section{Perceived control}

Before $S$ s in the control conditions were given control, there were no significant effects of MOOD, CON or MOOD x CON interaction. Following the manipulation of control, perceptions of control rose sharply (see figure 3). A MANOVA trend analysis indicated higher mean levels of perceived control in the control conditions $\left(t=13.2, p<10^{-3}\right)$, and lower perceptions of control in the depressed mood conditions $(t=-1.72, p=0.047)$. The CON $x$ MOOD interaction was n.s. The linear and quadratic trends were significantly influenced by mood $(t=1.92, p=0.06 ; t=-2.26, p=0.03)$, reflecting the sharper rise in perceived control in the elated group.

Consequently, the hypothesized effects of mood on perception of control were observed: when depressed, $S$ s raised their ratings of control more slowly than when they were elated. However, within the no control conditions there was no effect of mood, which was possibly due to a floor effect.

\section{Expectations of control}

These followed the same pattern as perceptions of control and are therefore not presented here. 


\section{Experienced pain}

With pretest subjective pain ratings and objective shock intensity as covariates, there was a significant effect of mood on experienced pain in the first block: elated $S \mathrm{~s}$ rated the shocks as less painful $(t=-1.86, p=0.035$; see figure 4). The CON effect was n.s. $(t=$ $1.27, p=0.21$ ), as was the CON $\times$ MOOD interaction $(t=-0.35, p=0.73)$.

During blocks $2-5$, the covariates were no longer significant and were therefore deleted. As hypothesized, there was a significant pain decreasing effect of control on the average pain ratings $(t=-2.29, p=0.014)$. Although the elated mood conditions were associated with lower pain ratings, the MOOD effect was not significant $(t=-1.07, p=0.15)$, possibly due to a floor effect in the elation/control condition, caused by the strong effect of exercising control. The MOOD x CON interaction was n.s. $(t=0.43, p=0.67)$. In order to test more directly the hypothesis that effects of mood on perceptions of control influence pain experiences, the amount of pain reduction from the first to the second block was analyzed separately for the control conditions only. During this second block, the strongest effect of mood on perceived control was observed in the control conditions (figure 2). The mood effect on pain reduction was $n_{\text {. }}$., however $(F(1,18)=0.76, p=0.40)$.

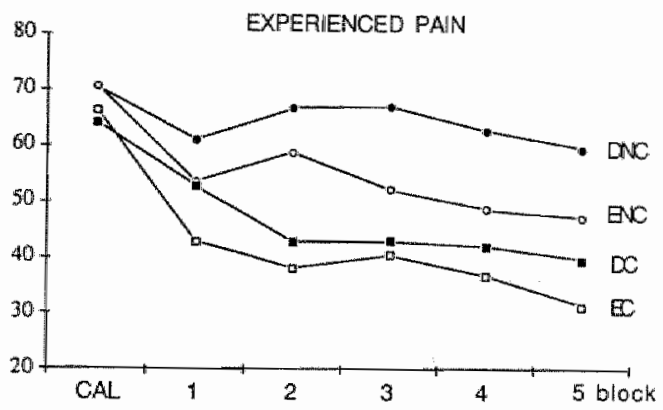

Figure 4. Experienced pain ratings, averaged in blocks of 4 trials. CAL denotes the pain rating in the calibration phase, in which the stimulation level was individually adjusted. When this CAL rating is controlled for, elated $S$ s rated the stimulus as significantly less painful than depressed $S s \quad(p=0.035)$. During the last 4 blocks, exercising control was related to lower pain ratings $(p=0.014)$. $\mathrm{EC}=$ elated mood induction/control; ENC $=$ elated $\operatorname{mood}$ induction/no control; $\mathrm{DC}=$ depressed mood induction/control; $\mathrm{DNC}=$ depressed mood induction/no control.

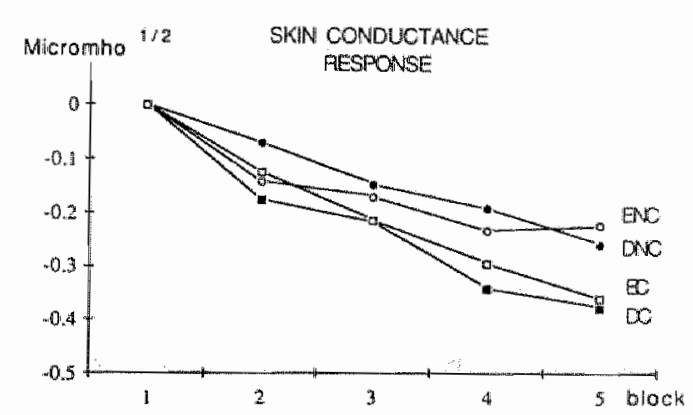

Figure 5. Skin conductance responses (SCRs), averaged in blocks of 4 trials, depicted as change scones from the first block. Raw SCRs were transformed by square root. With control, SCRs decreased faster than without control $(p=0.035) . \quad \mathrm{EC}=$ elated mood induction/control; $\mathrm{ENC}=$ elated mood induction/no control; $\mathrm{DC}=$ depressed mood induction/control; $\mathrm{DNC}=$ depressed $\operatorname{mood}$ induction/no control.

In sum, exercising control over the end of the painful stimulus had a pain decreasing effect. In contradiction with the hypothesis, however, there was no indication that mood has any effect on experienced pain by influencing perceptions of control or expectations of control. Firstly, there was an effect of mood on pain experiences, but not on perceptions of control, during the first block, before the controllability manipulation, and during the 
rest of the experiment in the no control conditions, which suggests that mood has an independent effect on pain. This mood effect is shown clearly by the correlation between mood ratings and pain experiences in the first block $\left(r=-.60 ; p<10^{4}\right)$. Secondly, in the instances in which mood infleenced perceptions of control, no additional effect on experienced pain was apparent.

\section{Skin Conductance Responses}

During the first block, there was a marginally significant MOOD $\times$ CON interaction, caused by stronger responding in the elated - no control condition $(F(1,35)=2.57, p=$ 0.12 ). Controlling for this initial difference (assuming that it was caused by individual differences), there was a significant CON effect on the average SCRs during block 2-5: as is shown in figure 5, SCRs decreased more with control than without control $(t=-1.88$, $p=0.035)$. There was no MOOD $(p=0.27)$, or MOOD x CON effect $(p=0.28)$.

\section{Mood: general}

Multivariate tests revealed a significant CON effect on the three moods $\left(F_{\text {Hot }}(3,35)=\right.$ $3.28, p=0.032$ ) during block $2-5$ when contrasted (as change scores) with the first block. There were no significant MOOD $(p=0.95)$ or MOOD $\times$ CON $(p=0.87)$ effects. Thus, exercising control significantly influenced $S \mathrm{~s}$ ' moods. Separate moods are presented below.

\section{Mood: depression - elation}

Despite the reduced effect of the music on depressive/elated mood during the pain stimulation (see figure 2; especially in the elated conditions mood dropped, $t=-3.55, p=$ $0.001)$, there was still a significant effect of the mood manipulation in the first block ( $t=$ $3.19, p<0.005$; pretest mood served as covariate) and in the blocks $2-5(t=2.36, p<$ 0.02 ; pretest mood served as covariate). In the first block, there were no significant CON or CON $x$ MOOD effects. During the last four blocks, there was a weak, marginally significant, $\mathrm{CON}$ effect in the expected direction $(t=1.29, p=0.10)$ : exercising control influenced mood positively. The MOOD $x$ CON interaction did not reach significance $(p=$ $0.73)$.

In sum, $S s$ found it difficult to remain as elated as immediately after the mood induction when the pain stimulation started. There is some evidence that having control influenced mood positively.

\section{Mood: nervousness}

There were no significant MOOD, CON or MOOD x CON effects on nervousness in the first block. During block 2-5, there was a significant positive effect of control on nervousness compared with the first block $(t=-2.15, p=0.04)$ : with control $S$ s became less nervous. However, when nervousness ratings were analyzed with the pretest score as covariate, the CON effect disappeared $(t=0.57, p=0.45)$. No MOOD and MOOD x CON effects were apparent. 
During the first block, there was still a MOOD effect on anger scores $(F(1,34)=4,66$; $p=0.04$; pretest anger served as covariate). Figure 6 shows that having control was related to decreases in anger during block $2-5$, whereas failing to acquire control was related to increases in anger $(t=3.02, p=0.005$; anger during block 1 served as covariate). However, with pretest anger as covariate the CON effect was no longer significant $(F(1,34)=$ $1.78, p=0.19)$. MOOD $(p=0.15)$ and MOOD $\mathrm{x}$ CON $(p=0.79)$ effects were n.s.

\section{Depressed mood and giving up}

In the depressed mood/control condition the "no code" button was not used at all; in the elated mood/no control condition as well as in the elated mood/control condition the mean number of presses on the "no code" button was 0.225 ; in the depressed mood/no control condition the mean number of presses was 0.625 . A non-parametric analysis of variance (Kruskal-Wallis) with the four conditions as groups revealed a significant group effect (corrected for ties: $\chi^{2}=10.07, p<0.02$ ). The depressed mood/no control condition differed significantly from the combined other three conditions (corrected for ties: $\chi^{2}=$ $8.04 ; p<0.005$ ), whereas there was no significant difference between these three conditions (corrected for ties: $\chi^{2}=3.57, p=0.17$ ). To summarize, in the depression/no control condition, $S s$ used the "no code" button more often than in the other conditions.

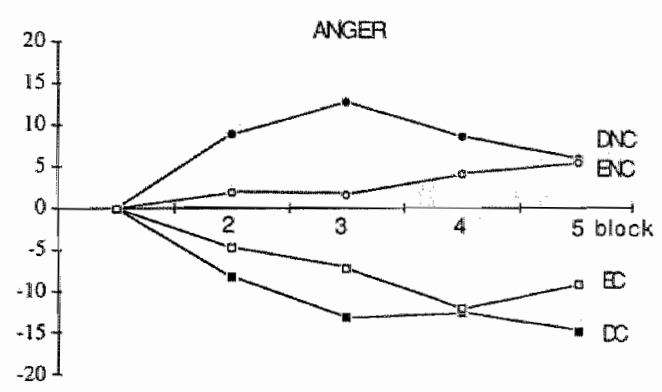

Figure 6. Ratings of anger, averaged in blocks of 4 trials, depicted as change scores from the first block. Exercising control was related to reductions in anger, whereas failing to acquire control resulted in increased anger $(p=0.005) . \mathrm{EC}=$ elated mood induction/control; $\mathrm{ENC}=$ ellated mood induction/no control; $\mathrm{DC}=$ depressed $\operatorname{mood}$ induction/control; $\mathrm{DNC}=$ depressed mood induction ino control.

\section{Controllability and pain predictions}

During block 2-5, there was no significant $\mathrm{CON}$ effect on the discrepancy between pain predictions and pain experiences $(F(1,36)=0.06, p=0.81)$ : without control, $S$ s did not overestimate pain more than with control. In accordance with the certainty hypothesis, Ss who exercised control were more certain about their pain predictions $(t=2.05, p=0.024)$. There were no MOOD or MOOD x CON effects. 


\section{Variables related to pain reduction}

In order to get more insight into factors that might be related to pain reduction, a series of regression analyses on the whole sample $(n=40)$ was executed. First, experienced pain served as dependent variable, objective shock intensity, pretest pain rating, elation-depression mood change with respect to pretest, change in nervousness with respect to pretest, the summed perceived control and expected control ratings ${ }^{2}$, and certainty rating as predictors. A backward procedure was employed. Results of the separate blocks 2-5, as well as of the awerage ratings during these blocks, all indicated two variables that were significantly related to lower pain experiences: perception/expectation of control and mood change (elated mood is related to less pain). The results of the averaged scores of blocks 25 are summarized in table 1 . The separate contributions of control and positive mood are also given in table 1 . Note that the overlap in explained variance is $0.1 \%$. Therefore, perceiving control and elated mood appear to have separate influences on the pain experience.

Table 1. Summary of regression analysils on experienced pain

\begin{tabular}{|c|c|c|c|}
\hline Predictor & $\beta$ & $t$ & $p$ \\
\hline \multicolumn{4}{|l|}{ not in equation } \\
\hline objective shock level (mA) & 0.20 & 1.50 & 0.14 \\
\hline pretest pain (VAS) & 0.11 & 0.72 & 0.48 \\
\hline mean certainty (VAS) & 0.10 & 0.69 & 0.50 \\
\hline mean change in nervousness (VAS) & -0.21 & -1.26 & 0.22 \\
\hline \multicolumn{4}{|l|}{ in equation } \\
\hline mean mood change (VAS) & -0.46 & -3.36 & 0.002 \\
\hline mean perceived/expected control (VAS) & -0.30 & -2.21 & 0.033 \\
\hline \multicolumn{4}{|c|}{$\begin{array}{l}\text { Explained variance }=32.2 \% \\
F(2,37)=8.39 ; p=0.001\end{array}$} \\
\hline \multicolumn{4}{|l|}{ separate contributions } \\
\hline mean mood change (VAS) & -0.47 & -3.28 & 0.002 \\
\hline \multicolumn{4}{|c|}{ Explained variance $=22.1 \%$} \\
\hline mean perceived/expected control (VAS) & -0.23 & -2.07 & 0.045 \\
\hline \multicolumn{4}{|c|}{ after entering anger ratings } \\
\hline objective shock level (mA) & 0.23 & 1.76 & 0.087 \\
\hline pretest pain (VAS) & 0.40 & 3.06 & 0.004 \\
\hline mean change in anger (VAS) & 0.57 & 4.49 & 0.001 \\
\hline \multicolumn{4}{|c|}{$\begin{array}{l}\text { Explained variance }=43.9 \% \\
F(3,36)=9.14, p=0.0001\end{array}$} \\
\hline
\end{tabular}

Results of backward regression analysis on the averaged pain ratings during block 2-5. Pretest pain, objective intensity, and mean ratings of perceived/expected control, certainty, nervousness, and elated mood served as predictors. Results indicate two independent variables relating to lower pain experiences: positive mood (change score with respect to pretest) and control. However, after entering anger (corrected for pretest rating), these variables were no longer significantly related to pain. Therefore, their contributors appear to be mediated by anger.

${ }^{2}$ Since the closely interrelated $(r=0.93)$ perceptions of control and expectations of control had similar contributions, these two variables were combined into a sum score. 
However, when anger ratings (change score with respect to pretest) were included as predictors, the equation changed dramatically: anger appeared to be strongly related to subjective pain (table 1). Elated mood and control disappeared from the equation, which suggests that their effects were mediated by anger.

Second, the mean SCR during block 2-5 was used as a dependent variable, with the averaged SCRs during the first block as an additional predictor, to control for initial differences. Results of the backward regression analysis are summarized in table 2.

Table 2. Summary of regression analysis on skin conductance responses

\begin{tabular}{lccc}
\hline Predictor & $\beta$ & $t$ & $p$ \\
\hline not in equation & & & \\
pretest pain (VAS) & -0.07 & -0.69 & 0.50 \\
objective shock level (mA) & 0.16 & 1.66 & 0.11 \\
mean certainty (VAS) & -0.15 & -1.47 & 0.15 \\
mean mood change (VAS) & -0.02 & -0.15 & 0.88 \\
mean change in anger (VAS) & 0.07 & 0.67 & 0.51 \\
mean change in nervousness (VAS) & 0.06 & 0.56 & 0.58 \\
in equation & 0.75 & 7.51 & $<10-4$ \\
mean SCR during block 1 & -0.24 & -2.43 & 0.02 \\
mean perceived/expected control (VAS) & Explained variance $=64 \%$ & \\
\multicolumn{5}{c}{$F(2,37)=32.15 ; p<10-4$} \\
contribution of certainty after deleting experienced/expected control & \\
mean SCR during blok 1 & 0.76 & 7.48 & $<10-4$ \\
mean certainty (VAS) & Explained variance $=62 \%$ & -2.02 & 0.05 \\
& $F(2,37)=29.99 ; p<10-4$ \\
\hline
\end{tabular}

Results of backward regression analysis on the SCRs (transformed by square root) during block 25. Pretest pain, objective intensity, mean SCR during block 1, and mean ratings of perceived/expected control, certainty, and elated mood served as predictors. Experienced/expected control and certainty have almost interchangeable contributions.

As can be seen in table 2, the perceived/expected control sum score is related to lower SCRs, when initial SCR differences are controlled for. Note that elated mood is not, nor the other subjective mood changes. The regression analysis indicated that subjective certainty could have an important contribution, overlapping with the contribution of perceived/expected control. Therefore, perceived/expected control was deleted from the analysis. The results (table 2) indicate that high certainty is indeed related to smaller SCRs. The explained variance of the two equations is almost equal ( $64 \%$ vs $62 \%$ ), which suggests that perceived/expected control and certainty play interchangeable roles in the regression equation.

\section{DISCUSSION}

There is clear evidence that control over pain has positive effects: the stimulus is experienced as less painful and physiological responding decreases more quickly. There is much weaker evidence that mood is influenced positively in the three dimensions, elation, nervousness and anger, when the $S$ has control compared to when the $S$ fails to acquire control. As hypothesized, mood influenced the perception of control: when given control, depressed $S$ s perceive control more slowly than elated Ss. However, there is not much 
evidence that mood influences the effects of control by influencing the perception of control. Whereas mood influenced the perception of control in the controllability conditions, it did not lead to different effects of control on experienced pain. In the no control conditions, the mood induction did not influence perceptions/expectations of control, but mood did influence the pain experience (also clearly evident from the first block). Therefore, there appears to be no evidence for a model of interaction between mood and perceptions of control. There is much more evidence for an additive model: mood and control have independent effects on experienced pain.

This leaves us with a paradoxical situation. On the one hand, it is generally assumed that the effects of control are mediated by perceiving control; on the other hand, the present findings suggest that the lower perceptions of control of the depressed $S$ s who exercised control have no negative consequences for the effects of control. There does not seem to be a relationship between the degree of subjectively perceived control and the effects of control. An explanation might be that the rating of perceived control in the controllability conditions was only superficially influenced by mood, but that the "true" perception of control was not. It should be noted that there was a very clear relationship between the control response and its effect in the present study. Therefore, the 'true" sense of control may have been more influenced by this clear response-effect relationship than by mood. With responses that have a less clear effect on the aversive stimulus, there may be more opportunity for mood to influence the 'true' sense of control. ${ }^{3}$

There was, however, also an indication for the hypothesized interaction: a clear effect of mood on the behavioural dimension of trying to find the controlling response. However, whereas depressed $S s$ made less attempts to find the controlling response, expectations of control were not lower than in the elated group. These findings therefore suggest that elated mood may compensate for the negative effects of failing to exercise control, without necessarily inducing an illusion of control.

The clinical meaning of these findings may be that mood does not influence the effects of having control - having control reduces the impact of aversive events in elated as well as in depressed mood - , but that depressed mood retards the perception of the $S$ that he/she is truly in control. Furthermore, there is evidence that when a $S$ is depressed, it is more difficult for him/her to persist in trying to acquire control. On the one hand, this is an encouraging finding: there seems to be no evidence that depressed patients cannot profit from exercising control. On the other hand, it will be a more difficult task to make them persist.

The certainty - distraction hypothesis of the effects of control was supported by the finding that $S s$ were more certain about the amount of pain they could expect when they had control. Although there was no direct test of the hypothesis that $S$ s who are certain that the pain will remain below unbearable limits distract their attention from the pain more easily, the present findings suggest that future tests of this hypothesis are indicated. This thypothesis, which is in fact an extension of Miller's minimax hypothesis, is not only attractive because of its parsimony, but also because it seems capable of connecting controllability theory with recent information processing theories, which stress the importance of attentional capacity ("central channel', 'working memory', 'central processor') in the processing of information. For instance, recent theoretical explanations of habituation

\footnotetext{
${ }^{3}$ Another possibility is that with a qualitatively different measurement of perceived control - asking for preceived control over experienced pain instead of perceived control over the offset of the pain stimulation - clearer results will be found.
} 
assume that when the representation of the stimulus remains in the working memory (and does not decay), stronger responding occurs and habituation is delayed (e.g. the Al state in Wagner's theory, see Wagner 1979; Donegan \& Wagner, 1987; see also Ohman, 1979). Especially SCRs are proposed as indices for the amount of central processing that is called for by the stimulus (Ohman, 1979). The finding that SCRs show a stronger decrease with control, supports the notion that less attention needs to be given to the stimulus with control. It is also understandable that perceptions/expectations of control were related to SCRs: the SCR occurs during the painful stimulation, before control could be exercised, and is therefore related to expected control. Therefore, these findings suggest that when the $S$ is highly certain about his/her expectation that control can be exercised effectively, less attention and information processing capacity is applied to the painful stimulus, which results in smaller SCRs. The interchangeable relationships of perceiving/ expecting control and subjective certainty with SCRs suggest that control has this physiological impact reducing effect by increasing certainty. Therefore, the following model is proposed with respect to the influence of controllability on SCRs:

\section{perceiving/expecting control $\rightarrow$ increased certainty $\rightarrow$ distraction $\rightarrow$ less processing of pain $\rightarrow$ decreased SCR.}

Although this explanation was supported with respect to SCRs, the findings with respect to subjective pain suggest that control and mood do not only exercise their influences via certainty. Having control seems to induce a state which can compensate for aversive experiences, as elated mood seems to do. The regression analyses suggests that this state is related to anger: high levels of anger (associated with failure to exercise control and with the depressed mood induction) were related to higher subjective pain, whereas low levels of anger (associated with exercising control and with the elated mood induction) were related to lower pain impact. It might be hypothesized that the ratings of anger are related to frustration (resulting from failing to find the controlling response), and that the effects of mood inducing stimuli and of uncontrollability are mediated as follows:

mood inducing stimuli

failure to exercise control

frustration/anger $\rightarrow$ increased pain impact

The effects of control are possibly mediated by a positive counterpart of frustration/ anger, perhaps something like a sense of 'mastery'. If this hypothesis is true, the model opens the interesting possibility that $S$ s could protect themselves from the negative effects of uncontrollability by stopping to try to acquire control. There are indeed indications that not trying to acquire control sometimes results in better adaptation than trying to acquire control in uncontrollable circumstances (Silver et al., 1982; Rothbaum et al., 1982).

This model supports Lang's (1985) view, partly based on a review of factor analytic studies of emotions, in which control is one of the three fundamental mood dimensions, independent of the dimension of evaluation (positive $v s$ negative feelings), and the dimension of activity. According to Lang, feelings of dominance, mastery, and control reflect a fundamental emotional dimension. It can be speculated that the concept of frustrative nonreward (Gray, 1975), pertaining to the negative effect of the absence of expected reward, is also related to it. According to Lang, anger is not only related to negative evaluation, but also to the control dimension. It can be speculated that anger results from failure to exercise the desired amount of control. In terms of Lang's model, the relation- 
ship between anger and subjective pain reflects the influence of two emotional dimensions on subjective pain: evaluation and control. Therefore, the present findings support the model that elated mood and control have independent pain decreasing influences.

In conclusion, mood and control seem to have independent (additive) subjective pain decreasing influences. Although depressed mood sometimes 'colours' perceptions of control, there is no evidence that the pain reducing effects of mood are mediated by these perceptions. In broad terms, mood seems to colour any evaluation (of pain, of expectations of control, of experiences of frustration/mastery, etc.), if there is room for a shift in evaluation (e.g. because of ambiguity). With respect to SCRs, perceiving/expecting control seems to reduce responses by increasing certainty, which possibly allows the $S$ to distract. With respect to subjective pain, the effects of (un)controllability seem to be mediated by anger. 


\section{SECTION 4.3. ATTENTION, NOT ANXIETY, INFLUENCES PAIN ${ }^{1}$}

\section{SUMMARY}

Four hypotheses about the influences of anxiety and attention on pain impact were tested in a critical experiment: (1) anxiety increases pain; (2) anxiety decreases pain; (3) attention to pain increases pain; (4) only the combination of anxiety and attention to pain increases pain (interaction hypothesis). In a $2 \times 2$ design, anxiety (low versus high) and attention (attention to versus distraction from the pain) were experimentally manipulated. Subjects received 20 electrically produced painful stimuli. Subjective pain experiences, skin conductance responses and heart rate responses gave no support for a pain impact increasing effect of anxiety. The anxiety-attention interaction hypothesis did not receive any support either. There was some support, only from the heart rate responses, that anxiety reduces pain impact. The critical factor appeared to be attention. Attention to the pain stimulus was related to a stronger pain impact (indicated by all measures) and to less subjective habituation, compared to distraction.

\section{INTRODUCTION}

It is still a controversial issue whether or not anxiety increases pain. Clinicians often seem to believe that anxiety intensifies pain (e.g., Chapman \& Turner, 1986). "This notion can also be found in theoretical views. For example, Melzack"s (1973) model on the psychological influences on pain states that anxiety increases pain. In the dual-process theory of habituation (Groves \& Thompson, 1970) it is stated that arousal increases the responses to a stimulus. Although the concept of arousal is not clearly defined in the dual-process. theory, anxiety could be hypothesized to increase the state of arousal, thereby intensifying pain. On the other hand, it has also been proposed that anxiety decreases pain. In an influential article, Bolles and Fanselow (1980) reasoned that fear and pain are mutually exclusive states. Fear is assumed to have priority, and therefore to reduce pain and activate the organism (flight-fight response), whereas pain is assumed to deactivate the organism, promoting healing. A possible mechanism of the pain reducing effects of anxiety may be endogenous opioid release. There are, indeed, animal studies (reviewed by Bolles and Fanselow, 1980) and anecdotal reports of severely wounded anxious human $S$ s who appear to feel no pain (e.g. Beecher, 1956), which seems to support the idea that anxiely has a pain-reducing effect.

As for physiological processes, both views can be defended. Chapman and Tumer (1986) argued that anxiety increases sympathetic activity and the release of epinephrine at the sympathetic terminals, which may sensitize or directly activate nociceptors. These authors have also suggested that increased muscle tension, which often accompanies anxiety, may cause additional pain, especially when muscles near the site of the source of the pain (e.g. the wound) are involved. On the other hand, there are physiological processes accompanying anxiety that may reduce pain, such as increased endogenous opioid

'Reprinted with permission from Behaviour Research and Therapy, 29, Arntz, A., Dreessen, L. \& Merckelbach, H., Attention, not anxiety, influence pain, 1991, Pergamon Press PLC. 
release (Thyer \& Matthews, 1986). Moreover, it is far from settled that reducing anxiety and physiological arousal, by relaxation or biofeedback for instance, directly causes pain reduction (Amtz \& Schmidt, 1989). Increasing physiological arousal may be as effective as decreasing arousal, as long as the $S$ believes in the effectiveness of the response (Holroyd et al., 1984; Bush et al., 1985).

Two types of studies have sought to illuminate the relationship between anxiety and pain in humans: correlational and experimental studies. As for the first type of studies, several studies have found that state anxiety and (experimentally caused or internal spontaneous) pain are correlated (Kleinknecht \& Bernstein, 1978; Dougher, 1979; Weisenberg et al., 1985; Ahles et al., 1987; Malow et al., 1987; Malow et al., 1989). However, many studies did not find significant relationships (Arntz et al., 1990a; Bowers, 1968; Chaves \& Brown, 1987; Klepac et al., 1980), or even negative correlations (Kent, 1984). Moreover, a positive correlation should not be confused with a causal effect of anxiety on pain: pain can, of course, be accompanied by anxiety and physiological arousal, but this leaves any causal direction open. Pain may as well lead to anxiety, or a third variable might be involved. Similarly, increased pain responses, as found in high trait anxious $S$ s (e.g., Dougher, 1979) may be caused by a third variable, and not by anxiety itself. To clarify the issue of causality, studies are needed in which anxiety is experimentally manipulated.

To the present authors' knowledge only six studies have investigated the effect of experimentally induced anxiety on pain. Unfortunately in two of these (Haslam, 1966; Bobey \& Davidson, 1970) the effectiveness of the anxiety induction was not assessed. Three studies did find (sometimes mixed) evidence for a pain sensitivity-increasing effect of anxiety (Haslam, 1966; Dougher et al., 1987; Cornwall \& Donderi, 1988), one failed to demonstrate any clear effect (Weisenberg et al., 1984), and the other two found indications for the opposite effect: anxiety decreased pain sensitivity (Bobey \& Davidson, 1970; Malow, 1981). Interestingly, in the Dougher et al. study only pain-related anxiety had pain sensitivity-increasing effects. Anxiety that was not related to pain did not lead to lower pain thresholds and lower pain tolerance levels. The Weisenberg et al. study also reports some evidence for this influence of focus of anxiety. However, in the study of Cornwall \& Donderi, pain-irrelevant anxiety had also a pain impact increasing effect. The main difference with anxiety manipulations in other studies is, that $S \mathrm{~s}$ in this condition were not confronted with an anxiety object during pain stimulation (the anxiety was induced by telling the $S$ s that hey would get a stressful interview after the pain test). Thus, the effect of anxiety on pain might be related to the presence $v s$ absence of an anxiety evoking stimulus during pain stimulation.

In summary, empirical consensus on the influence of anxiety on pain is lacking. One reason for this might be that a third, hitherto overlooked, factor modulates the relationship between anxiety and pain. One obvious candidate in this respect is attention. In most studies possible attentional effects were not controlled for. There is evidence that attention towards pain increases the pain experience, whereas distraction reduces it (Arntz \& Schmidt, 1989; Kanfer \& Goldfoot, 1966; Grimm \& Kanfer, 1976; Worthington, 1978; Beers \& Karoly, 1979; Tan, 1982; Turk et al., 1983). Therefore, it seems evident that if the focus of fear/anxiety is directed away from the pain, the pain experience is reduced; and conversely, if the pain itself is focused upon because of pain-related fear/anxiety, the pain experience will be stronger. The influence of such an attention factor might explain why some studies found a positive anxiety-pain relationship, whereas other studies found the opposite to be true. Thus, in the empirical studies on the influence of anxiety on pain, anxiety may have been confounded with attention. Therefore, it is worthwhile to re-examine the influence of anxiety on pain, controlling for the effects of attention. 
The present study was designed as a critical experiment of several hypotheses that can be put forward with regard to the influence of anxiety and attention on pain. $A 2 \times 2$ design was employed, the first factor being anxiety (low ws high), and the second being attention (towards pain (attention) ws towards an extemal object (distraction)). In the low anxietyl attention condition, $S_{s}$ were given non-threatening information about the stimulus and were asked to concentrate on the pain stimulus as much as possible. In the high anxiety/ attention condition, $S s$ were made anxious for the stimulus and were instructed to concentrate on the stimulus. In the low anxiety/ distraction condition, $S s$ were distracted and were instructed to disregard the stimulus as much as possible. In the high anxiety/distraction condition, $S$ s participated in an exposure in vivo procedure with a phobic object (spider) and were instructed to disregard the pain stimulus as much as possible. A similar experiment has been done by Weisenberg et al. (1984), but this study suffers from some methodological inadequacies, such as too small cell sizes, unclear tests of the hypothesis, and failure to demonstrate that $S$ s in the pain-attention conditions attended more to the pain than $S$ s in the pain-distraction conditions. Moreover, this study failed to demonstrate clear effects of attention, possibly due to the weak manipulation of attention.

Four hypotheses were tested:

1. the hypothesis that anxiety, irrespective of the focus of attention, increases pain impact.

2 the Bolles-Fanselow hypothesis, which would be supported if, irrespective of the focus of attention, anxiety would decrease pain impact.

3. the hypothesis that attention, irrespective of anxiety, is related to pain impact.

4. the possibility of an interaction was also considered. When pain impact is larger only when the $S$ is anxious and attention is directed towards the pain, this view would be supported:

Two types of pain measures were employed: subjective pain experience and physiological responses to pain. In many studies, threshold and tolerance/endurance levels are measured, which have a clear behavioral component (especially tolerance and endurance, which are related to escape from the pain stimulation). It should be noted, however, that the correlation between subjective and physiological pain impact responses on the one hand, and pain behaviour on the other hand, is generally low. In the present context, the influence of anxiety and attention on pain impact seemed to be most relevant.

\section{METHOD}

\section{Subjects}

Because of the anxiety manipulation used in the high anxiety/distraction condition (exposure to spiders), it was decided to use spider phobics as $S$ s not only in this condition, but, to secure comparability, in all four conditions. They were recruited by means of announcements at publicity boards in several university buildings, in the local hospital, and in the local library. Before starting a session, procedural information was given and $5 s$ signed statements of informed consent. Subjects with pain complaints or with serious cardiac trouble were excluded, as were pregnant $S \mathrm{~s}$. A small remuneration was given for participating. There were 52 women and $3 \mathrm{men}$ in the final sample (see below). Mean age was 26 yrs (range 17-56). The mean score on the Spider Phobia Questionnaire (SPQ, Klorman et al., 1974) was 16.3, which indicates that this sample was definitively phobic when American and Swedish norms are considered (Klorman et al., 1974; Fredrikson, $1983)$. 


\section{Materials}

Anxiety as experienced during the shock and experienced pain were measured by means of $10 \mathrm{~cm}$. Visual Analogue Scalles (VASs). Fear of spiders was assessed by means of the Spider Phobia Questionnaire (SPQ, Klorman et al, 1974; Fredrikson, 1983). After the last trial, $S$ s rated degree of attention given to the pain during stimulation on a $10 \mathrm{~cm}$. VAS. An open question requested to rnake a note of the main focus of attention during the experiment. In the low anxiety/distraction condition, $S s$ were given a video tape as distractor. The Ss could choose between a nature movie (without spiders and insects), an episode of L.A. Law, and a performance by a Dutch cabaret artist.

\section{Apparatus and Physiological Recording}

Apparatus was placed in an adjacent room. Skin Conductance Responses (SCRs) and Skin Conductance Level (SCL) were measured by a Beckman Skin Conductance Coupler (type 9844), using the method of constant voltage ( 0.5 Volts). The coupler allowed for a maximum sensitivity of 0.05 micromho. Electrodes were attached to the medial phalanges of the second and third fingers of the non-dominant hand. The highest deflection within the $10 \mathrm{sec}$ stimulation period and the $10 \mathrm{sec}$ post-stimulus period was measured. SCRs were square root transformed (cf. Levey, 1980) and range corrected (Lykken, 1972).

Heart rate was measured by a Beckman Voltage/Pressure/Volume Coupler (type 9853A). Heart rate was measured via electrodes placed below the sternum and at the left edge of the chest. The signal was first filtered through a low-pass filter (set at $40 \mathrm{~Hz}$.), in order to reduce noise due to the shock, before being led to a microprocessor for heartbeat detection. Interbeat intervals were transformed to heartbeats (per min) on a second by second basis, employing weights corresponding to the amount of the second occupied by the interbeat interval (Graham, 1978).

The pain stimulation was produced by means of a Siemens Neuroton 627, passed for use with patients. The painful shock was delivered at the ankle of the $S$ via a concentric shock electrode (Tursky, 1974). The stimulation was raised during two sec to the preselected level, remaining at that level for $6 \mathrm{sec}$, and was returned to zero during $2 \mathrm{sec}$. This stimulation produces a painful, stinging sensation, which is unlike electric shocks as known from barbed wire, electric mains, etc. The intertrial intervals varied randomly from 21 to $59 \mathrm{sec}$ (mean $40 \mathrm{sec}$ ).

A microcomputer (PDP Minc-11) controlled the pain stimulation, intertrial intervals, and registration.

\section{Procedure}

Subjects were randomly allocated to one of the four conditions. After obtaining informed consent, the electrodes were fastened. In case of the high anxiety/distraction condition (see below), a hierarchy of spider anxiety related items was constructed. Next, shock level was established by increasing the amperage from zero up in steps of $0.2 \mathrm{~mA}$., until the $S$ indicated that the shock was painful and rated it approx 60 on a $0-100$ VAS. This level was kept constant over trials. Following the selection of the shock level, Ss were asked to relax for $5 \mathrm{~min}$, at the end of which a $15 \mathrm{sec}$ baseline measurement was done. After this measurement, the second author entered the room, announced that the series would start (without mentioning the number of trials or the duration of the series), 
and instructed the $S$ to fill in the VASs after each pain stimulation. A loose-leaf file contained VASs for more than 50 trials.

In the low anxiety/attention condition, $S s$ were instructed to concentrate as much as possible on the pain stimulation, and to try and find descriptions of the local sensations evoked by it. Subjects were instructed that they would be interviewed after the series about their experiences. It was also made clear to the $S$ that the stimulus was entirely safe, produced by medical apparatus used by neurologists and physiotherapists. After these instructions, the experimenter left the room and the $S$ started with the task.

In the low anxiety/distraction condition, $S$ s were instructed to concentrate as much as possible on the video, and try not to let themselves be distracted by the pain stimulus. The Ss were told that they already were familiar with the stimulus, suggesting that it was unnecessary to give any attention to it. As in the other low anxiety condition, it was attempted to make clear to the $S$ that the pain stimulus was safe, being produced by medical apparatus, etc. After these instructions, the experimenter left the room and the $S$ started with the task.

Pilot studies on different ways to induce anxieiy for the pain stimulus indicated that none of them was reliably effective for all $S$ s Consequently, the two most effective methods were chosen (described below as 'first version' and 'second version' of the high anxiety/attention condition) and both were run. More $S$ s were run than in the other conditions, because it was anticipated that we would fail to induce anxiety in approx. half of the Ss. Note that anxiety is not a dependent measure in the present experiment, but a necessary condition for the high anxiety conditions.

In the first version of the high anxiety/attention condition, $S s \quad(n=13)$ were given exactly the same information and instructions as in the low anxiety/attention condition. Via the intercom, the start of the series was announced. The intercom was not switched off, and several personal remarks about the $S$ were made by the experimenters in order to enhance credibility of the manipulation. It was intended to suggest that the experimenters had forgotten to switch off the intercom. After these personal remarks, a tape was played via loudspeakers in the control room. The $S$ could hear the conversation on the tape via the intercom. The conversation contained various non-veridical alarming topics, such as: technical troubles with the shocker; disturbances of the heart rate caused by the shocker and wrong connection of the apparatus; troubles with the capacitor of the shocker, which might discharge itself on the $S$, leading a technician, who dropped in, to insist on immediate discontinuation of the experiment; the application of the wrong type of paste in the shock electrode, possibly leading the shock to burn the skin, etc.

In the second high anxiety/attention version, $S s(n=14)$ were given the same attention instruction as in the low anxiety/attention condition. After baseline measurement, the second author told the $S$ that some shocks might become suddenly very strong at the moment that the standard shock would decrease to null. To increase credibility and anxiety, an example of such a shock was given. The $S$ was told that a random generator in the computer would decide whether this would occur, that every shock could suddenly become stronger, and that if there would be several shocks without a sudden increase, this would not mean that this would decrease the possibility of a sudden increase (the analogy of throwing a dice was made).

In the high anxiety/distraction condition, the second author started the exposure after having given the instructions about the VASs. There were two or three types of spiders available in order to control the level of exposure. It was attempted to dose the exposure at a subjective level of anxiety of appr. 50 on the 0-100 VAS. Subjects were instructed to attend to the spider, and to try not to let themselves be distracted by the pain stimulus. 
Following the series of 20 pain stimuli, Ss in all four conditions rated degree of attenthon paid to the stimulus during stimulation, and indicated main topics of attention during the series. After carefully debriefing the $S$, a $15 \mathrm{sec}$ baseline measurement was obtained. Finally, the $S$ filled out the SPQ. Electrodes were then removed, the $S$ was paid and thanked for participating.

\section{Data reduction and analysis}

Inspection of the heart rate patterns in the four conditions revealed that a HR acceleration took place after shock offset. Heart rate responses of each trial were therefore computed as the difference between the mean HR during 9 sec after shock and the mean HR during $9 \mathrm{sec}$ before the shock. Subjective pain ratings, SCRs and HRRs were averaged in blocks of 4 trials, and subsequently analyzed by means of a MANOVA trend analysis. Two trends were inspected: the mean trend (reflecting the average response) and the linear trend (as a measure of habituation). In case of the SCRs, the linear trend with the log of the trial number was used to test for differences in exponential decrease. Two covariates were employed: pretest subjective pain as indicated on the VAS and objective pain level (in $\mathrm{mA}$ ). In case of SCRs, SCL served as additional covariate. The covariates were deleted if their contribution was n.s. $(p>0.10)$. The SPSSX MANOVA output yields $t$-tests, which allow for directional interpretation (these $t$-tests of course result in the same levels of significance as $F$-tests when interpreted two-tailed). The anxiety effect was tested twotailed, because two opposite hypotheses were formulated (anxiety increases pain; anxiety decreases pain). The attention effect was tested one-tailed, because a directional hypothesis was formulated for the effect of attention.

\section{RESULTS}

\section{Manipulation check and selection of subjects}

Since the anxiety induction in the high anxiety/attention condition had rather different effects on the various $S s$, the median of the anxiety ratings over all $S$ s was used as a criterion for $S$ selection in this condition. $S$ s who had a mean anxiety rating that was smaller than the median (16.35 on the 0-100 VAS) were excluded from the high anxiety condition (when this criterion is used, none of the $S$ s who participated in the spider exposure has to be excluded). Similarly, distraction as measured by a post-test VAS appeared to have been very modest in some $S s$ in the low anxiety/distraction condition. Therefore, the median of this VAS over all $S \mathrm{~s}(62.50)$ was used as a criterion for $S$ selection. Of the 72 original $S s$, 17 were excluded, 5 from the low anxiety/distraction condition because they attended too much to the pain stimulus and 12 from the high anxiety/attention condition because of failure to induce anxiety in them. Table 1 displays the final $n$ 's.

An ANOVA on the post-test attention ratings (table 1) revealed that $S \mathrm{~s}$ in the distraction conditions rated a smaller amount of attention paid to the pain stimulation than $S s$ in the attention conditions $\left(t=10.94, p<10^{-3}\right)$. The anxiety main effect was $n . s .(t=0.66, p=$ $0.51)$, as was the anxiety $\mathrm{x}$ attention interaction $(t=-0.10, p=0.92)$. At the open question, all $S s$ in the distraction conditions named subjects not related to the pain stimulus, whereas all but one $S \mathrm{~s}$ in the attention conditions named pain stimulus-related topics. 
An ANOVA on the averaged anxiety ratings (table 1) revealed a significant effect of anxiety induction $\left(t=8.13, p<10^{-3}\right)$. The main effect of attention vs distraction was $n . s_{\text {. }}$ $(t=1.06, p=0.29)$, as was the interaction $(t=1.91, p=0.06)$.

Table 1. Mean attemtion and anxiety ratings in the four conditions

\begin{tabular}{lccccc}
\hline Condition & \multicolumn{2}{c}{ attention } & \multicolumn{2}{c}{ anxiery } & $n$ \\
& mean & $5 . d$. & mean & $s . d$ & \\
\hline Low anxiety/attention & 83 & $(17)$ & 12 & $(11)$ & 13 \\
Low anxiety/distraction & 31 & $(18)$ & 9 & $(12)$ & 14 \\
High anxiety/attention & 80 & $(11)$ & 35 & $(15)$ & 15 \\
High anxiety/distraction & 28 & $(23)$ & 46 & $(15)$ & 13 \\
\hline
\end{tabular}

Attention to pain was assessed by means of a posttest VAS (0-100), anxiety by means of VASs $(0$ 100) filled in after each trial the averaged score is presented here).

\section{Subjective pain ratings}

A MANOVA trend analysis revealed that there was a significant attention effect on the mean pain rating as well as on the linear trend (table 2). Distraction was related to lower pain ratings and to habituation (figure 1), whereas attention was related to higher pain ratings and absence of habituation. The anxiety effect was n.s., as was the anxiety $\mathrm{x}$ attention interaction (table 2). Thus, these results support the hypothesis that only attention is related to the experience of pain, whereas anxiety has no effect.

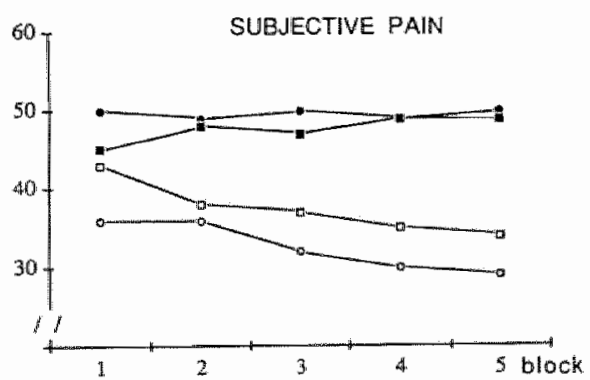

Figure 1. Subjective VAS ratings of the pain stimulus. Ratings are averaged over blocks of four trials. Attention to the pain stimulus was clearly related to higher pain ratings and lack of habituation, whereas distraction was related to lower pain ratings and habituation. $(L A=$ low anxiety; $H A=$ high anxiety; $A$ $=$ attention directed to the pain stimulus; $D$ = distraction from the pain stimulus).

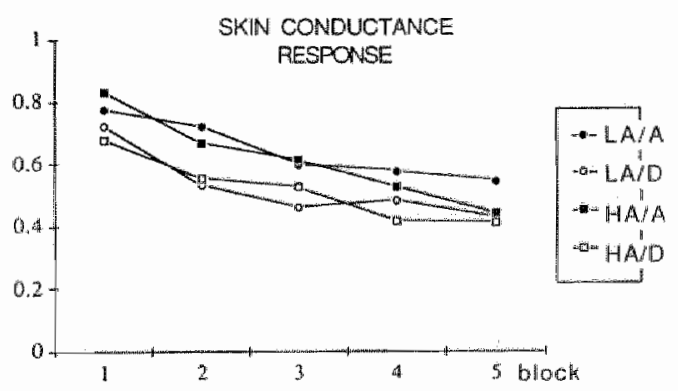

Figure 2. Skin conductance responses (SCRs) to the pain stimulus. SCRs were transformed by square root, range corrected and averaged over blocks of 4 trials. Distraction was relatad to smaller SCRs. (LA = low anxiety; HA $=$ high anxiety; $\mathrm{A}=$ attention directed to the pain stimulus; $D=$ distraction from the pain stimulus). 
Table 2. Trend analysis on subjective pain ratings

experimental factorcowariates

\begin{tabular}{lllllllllll}
\hline & anxiety & \multicolumn{2}{c}{ attention } & \multicolumn{2}{c}{$\begin{array}{l}\text { anxiety } x \\
\text { attention }\end{array}$} & \multicolumn{2}{l}{$\begin{array}{l}\text { pretest } \\
\text { pain }\end{array}$} & amperage \\
\hline Trend & $t$ & $p$ & $t$ & $p$ & $t$ & $p$ & $t$ & $p$ & $t p$ \\
\hline mean & 0.75 & 0.46 & 2.26 & 0.014 & -0.85 & 0.40 & 3.01 & 0.004 & -1.030 .31 \\
linear & 0.22 & 0.83 & 1.79 & 0.04 & 0.48 & 0.63 & 1.53 & 0.13 & 0.690 .49 \\
\hline
\end{tabular}

The anxiety effect was tested two-tailed, the attention effect one-tailed.

\section{Skin Conductance}

An ANOVA on the SCL during the series, with pretest baseline $S C L$ as covariate, revealed a significant main effect of anxiety: in both high anxiety conditions, SCL was significantly higher than in the low anxiety conditions $(t=2.21, p=0.03)$. The attention main effect was n.s. $(t=-0.78, p=0.44)$, as was the anxiety $\mathrm{x}$ attention interaction $(t=1.15, p=$ 0.26 ). The anxiety effect should, of course, be attributed to the anxiety manipulations.

A MANOVA trend analysis on the SCRs revealed a significant attention effect on the mean SCR: attention to pain was related to stronger SCRs $(t=2.34 ; p=0.01)$ than distraction from pain. The anxiety and interaction effects were n.s. (figure 2 , table 3 ). There were no significant differences with regard to SCR habituation. To summarize, there was clear evidence that attention was related to stronger SCRs, whereas anxiety appeared to have no effect at all on SCRs to pain. ${ }^{2}$

Table 3. Trend analysis on SCRs

\begin{tabular}{lllllllllllll}
\hline & \multicolumn{3}{c}{ experimental factor } & \multicolumn{3}{c}{ covariates } \\
\hline & anxiety & attention & $\begin{array}{l}\text { anxiety } x \\
\text { attention }\end{array}$ & $S C L$ & \multicolumn{1}{l}{$\begin{array}{l}\text { pretest } \\
\text { pain }\end{array}$} & amperage \\
\hline Trend & $t$ & $p$ & $t$ & $p$ & $t$ & $p$ & $t$ & $p$ & $t$ & $p$ & $t$ & $p$ \\
\hline mean & -0.36 & 0.72 & 2.34 & 0.01 & -0.21 & 0.84 & 1.30 & 0.20 & -1.00 & 0.32 & -1.03 & 0.31 \\
linear & -0.94 & 0.35 & -0.53 & 0.70 & -1.10 & 0.28 & 0.57 & 0.57 & -0.07 & 0.95 & 0.69 & 0.49 \\
\hline
\end{tabular}

The anxiety effect was tested two-tailed, the attention effect one-tailed.

\section{Heart rate}

Inspection of the heart rate patterns of the 4 groups showed that acceleration took place after the shock. According to Turpin (1986), this long latency acceleration reflects a defense reflex, and must be distinguished from early acceleration, reflecting a startle reflex. A MANOVA trend analysis on the heart rate accelerations revealed marginally significant effects of anxiety (anxious $S$ s showed on the average less HR acceleration than non anxious $S$ s, figure $3 \&$ table 4 ) and attention (attention to pain was related to stronger HR

\footnotetext{
${ }^{2}$ The first SCR to the pain stimulus was also analyzed. Results were similar to those of the maximum deflection: attention was related to stronger SCRs $(t=2.22, p=0.015)$, anxiety did not relate to SCRs $(t=-0.91, p=0.34)$, and the interaction effect was also $n . s .(t=0.21, p=0.84)$.
} 
acceleration than distraction). The interaction was $n . s$. There were no significant anxiety or attention effects on HRR habituation (table 4).

Mean heort rate accelerotion

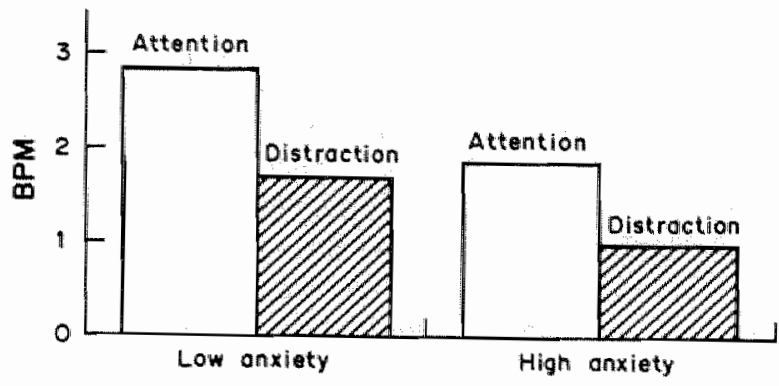

Figure 3. Heart rate responses (HRRs) to the pain stimulus averaged over 20 trials. HRRs were computed as the mean HR 9 sec after the pain stimulus, subtracted from mean HR during the 9 sec pretriall period. Distraction, as well as anxiety, were related to smaller HRRs (both marginally significant).

Table 4 Trend analysis on heart rate responses

\begin{tabular}{|c|c|c|c|c|c|}
\hline \multirow[b]{3}{*}{ Trend } & \multicolumn{3}{|c|}{ experimental factor } & \multicolumn{2}{|c|}{ covariates } \\
\hline & anxieby & attention & $\begin{array}{l}\text { anxiety } x \\
\text { attention }\end{array}$ & $\begin{array}{l}\text { pretest } \\
\text { pain }\end{array}$ & amperage \\
\hline & $p$ & $p$ & $p$ & $p$ & $p$ \\
\hline mean & -1.690 .10 & 1.380 .09 & $-0.23 \quad 0.82$ & 1.380 .18 & $-0.16 \quad 0.88$ \\
\hline \inear & -0.010 .99 & $-0.46 \quad 0.67$ & $\begin{array}{lll}1.02 & 0.32\end{array}$ & -2.100 .04 & $-0.05 \quad 0.96$ \\
\hline
\end{tabular}

The anxiety effect w/as tested two-tailed, the attention effect one-tailed.

The anxiety effect on HRRs could be attributed to increased mean pretrial HR in the high anxiety conditions, caused by the anxiety manipulations. If the difference between baseline HR and mean pretrial HR is employed as a covariate, the anxiety effect shrinks to a n.s. level $(t=-1.28$, $p=0.21$, whereas the attention effect remains essentally the same $(t=1.31, p=0.10)$. Thus, the anxiety effect may have been caused by the effect of the anxiety manipulation on tonic $H R$.

\section{Are the results dependent on the selection of subjects?}

It could be argued that the observed distraction effects result from selecting $S$ s with low attention scores in the low anxiety/distraction condition: the high attention scores of the removed $S s$ may have been caused by a strong pain experience. The remaining $S s$, likewise, may have had low attention scores because of low pain. Thus, instead of distraction causing lower pain impact, lower pain impact may have led to distraction. To control for this possible artefact, all analyses were repeated without selecting $S$ s on the basis of their attention VAS scores $(n=60)$. However, the results appeared to be very robust: distraction from pain was still related to a smaller experience of pain $(t=2.25, p=0.01)$, to slower 
subjective habituation $(t=2.03, p=0.02)$, to smaller SCRs $(t=2.03 ; p=0.02)$ and (marginally significant) to smaller HRRs $(t=1.60, p=0.06$ ). The anxiety and interaction effects also remained the same. Thus, the effect of attention versus distraction appeared to be no artefact of the $S$ selection procedure, but to depend on the availability of a distractor. Since only anxious $S s$ were selected in the high anxiety conditions, it is unlikely that the observed lack of effect of anxiety can be attributed to $S$ selection. The analyses over the whole sample $(n=72)^{3}$ indeed revealed that distraction from pain was related to smaller subjective pain responses $(t=-1.94 ; p=0.028)$, faster habituation $(t=-1.47 ; p=0.072)$, and smaller SCRs $(t=-2.10 ; p=0.02)$. The effects of anxiety were n.s. (mean subjective pain rating: $t=-0.42, p=0,68$; subjective pain habituation: $t=-0.17, p=0.87$; mean SCR: $t=-$ $0.01, p=0.99$ ), as were the interaction effects (mean subjective pain rating: $t=0.64$, $p=0.53$; subjective pain habituation: $t=0.64, p=0.53$; subjective pain habituation: $t=$ $0.35, p=0.73$; mean SCR: $t=-0.49, p=0.62$ ). Thus, the lack of effect of anxiety cannot be attributed to $S$ selection either. The conclusion that anxiety has no influence on pain as derived from analyzing the whole sample can, however, be critized because of failure to induce anxiety in many $S$ s in the high anxiety/attention condition. Therefore, the selection of $S$ s who were successfully made anxious seems to give a more valid base for testing the effects of anxiety.

\section{DISCUSSION}

Three unambiguous conclusions can be drawn from the data presented above. First, attention to pain is clearly related to stronger pain responses. Second, results do not support the hypothesis that anxiety is related to stronger pain responses. Third, the opposite theory, that anxiety is related to less responding to pain, did not receive much support either. Only heart rate responses appeared to be slightly less in the anxiety conditions. This effect may, however, have been caused by the influence of the anxiety manipulation on the tonic HR level (law of initial values). It seems difficult to attribute the lack of influence of anxiety on pain to too low levels of anxiety: especially in the high anxiety/ distraction condition, the anxiety was relatively high and 'real' because it was induced by means of exposure to a phobic object. There were, however, no indications whatsoever that pain responses were more strongly influenced in this condition.

Attention appears to be the critical factor: subjective pain, SCRs and (marginally significant) HRRs were less when the $S$ directed his/her attention away from the pain stimulation. The importance of attention is perhaps most clearly shown by the subjective pain ratings: when attention is focused upon the pain, there appears to be virtually no subjective habituation at all (figure 1). Distraction, on the other hand, appears to be related to ongoing habituation. The present data suggest that it might be fruitful and clinically relevant to examine the effect of distraction on long term habituation of pain responses.

The present study opens the possibility that the contradicting findings found in previous research on the influence of anxiety on pain results from the operation of the focus of

${ }^{3}$ The heart rate data of the Ss who failed to get anxious were not further processed. Because of the relatively wak effects of the experimental factors on the HRRs, this seemed to be not very relevant. An additional check revealed that the removed $S s$ of the high anxiety/attention condition did not differ from the selected $S s$ of this condition with respect to mean experienced pain $(F(1,25)=$ $1.09, m . s$.$) and SCRs (F(1,25)<1.00)$. Therefore, the selected high anxiety/attention $S$ s were not anxious because their pain responses were stronger. 
attention as a third factor. For instance, in the Malow (1981) study, experimentally induced anxiety was found to be related to lower pain reports and reduced pain discrimination ability. These effects may have been caused by the attention diverting effect of the anxiety induction manipulation, which was accomphished by warning the $S$ with a red light on a shock apparatus: the red light may have diverted the $S$ s attention from the pain, and therefore reduced pain report and pain discrimination ability. Similarly, the anxiety induction in the Bobey and Davidson (1970) study (letting the $S$ s listen to a tape with cries, screams and moans) may have diverted the $S$ 's attention away from the pain and therefore caused higher pain tolerance levels. With respect to correlational studies which measured state or trait anxiety before the pain application, the present findings suggest that positive anxiety - pain correlations might have been caused either by response tendency (high anxious $S$ s showing a negative tendency in rating any stimulus), or to the different use of attention diverting strategies in high $w$ low anxious $S$ s. The hypothesis that anxious $S$ s attend more to pain, or find it more difficult to avert their attention from pain, as long as there is no other powerful distracting stimulus, might be worthwhile to investigate. A study by Cornwall and Donderi (1988) seems to give some support for this hypothesis: $S$ s who were made anxious for a stressful interview, to be conducted after the pain test, showed elevated heart rate and elevated pain ratings during stimulation, compared to a control group. However, the majority of measures (pain threshold, pain tolerance, EMG, facial grimaces, and pain rated after the pain stimulation) were n.s. different from the control group. Moreover, attentional effects were not controlled for. Thus, a more direct test of the hypothesis that anxious $S$ s have more difficulties to distract from pain as long as there is no powerful distracting stimulus, would be welcome.

The lack of influence of anxiety does not mean that anxiety is never relevant with respect to clinical pain. When the $S$ is anxious about the causes or the course of pain, he/she may pay a lot of attention to the pain, thereby increasing the pain. However, the present study indicates that in such instances the critical pain increasing factor is attention, rather than anxiety or anxiety-related arousal. Pain-related anxiety merely motivates the $S$ to direct attention to the pain. Anxiety might also produce pain via anxiety-induced muscle tension, which can produce muscle aches. Clearly, this mechanism was not addressed in the present study. However, muscle tension will play no part at all in many cases of pain.

It is interesting to note that the present findings seem to contradict Groves and Thompson's dual-process theory (1970) of habituation. Groves and Thompson state that two processes play a part in habituation: firstly, a decremental one, taking place in the direct connection between stimulus receptor and response effector (causing habituation); and secondly, an incremental process (causing sensitization), which reflects the general 'state" of the organism. More specifically, Groves and Thompson state that arousal (excitation', "activation", etc.) is the key concept in this second process." high arousal is related to stronger responding (and can therefore cause dishabituation, sensitization and delayed habituation). The present study indicates, however, that anxiety induced arousal is not related to stronger subjective and autonomic responses to pain, nor to delayed habituation. Instead of anxiety or arousal, attention seems to be the important state variable. It should be noted, however, that Groves and Thompson's work is based primarily on the study of motor responses, and therefore should not necessarily apply to autonomic or subjective responses. It might be interesting to investigate anxiety and attention effects on human motor responses to pain.

Although of considerable theoretical importance, the relationships between anxiety, attention and pain are not without practical implications. The present findings strongly indicate that strategies to distract attention from pain (as already widely practised by clini- 
cians e.g the G.P. who distracts the child's attention from a painful intervention by telling a nonsense story, asking the child to correct the story ${ }^{4}$ ) seem to be more indicated than techniques or medicines for anxiety reduction. Nevertheless, when anxiety is the motivating factor in attending to pain, anxiety reduction might be helpful. In any case, anxiety reduction seems not to be contraindicated, as the Bolles-Fanselow hypothesis leads one to believe. On the contrary, the present findings suggest that anxiety reduction does not lead to an increased pain experience, as long as attention is not focused more on the pain. Moreover, reduction of suffering due to anxiety might be a legitimate goal in itself, which will not necessarily be in conflict with the goal of reducing suffering due to pain. With regard to chronic pain, the present findings suggest that attention-diverting techniques might be a powerful. ingredient of psychological treatment, because distraction immediately decreases the experienced pain, and seems to promote subjective habituation. In addition, the experience to be able to master the attention-attracting properties of pain might be very important for chronic pain patients and may serve to reduce feelings of helplessness and increase a sense of control over the pain and life in general.

"See also: Beers \& Karoly (1979); Grimm \& Kanfer (1976); Kanfer \& Goldfoot (1966); Miller \& Grant; Tan (1982); Turk et al. (1983) for spontaneous and instructed use of distraction as a pain coping strategy. 


\section{SECTION 4.4. ANXIETY, ATTENTION, AND PAIN ${ }^{1}$}

\section{SUMMARY}

In a within $S$ design the hypothesis was tested that focus of attention is a major factor mediating the influence of anxiety on pain. The hypothesis states that if anxiety leads the $S$ to direct attention to the painful stimulus, pain responses are increased; but if anxiety leads the $S$ to direct attention away from pain (for instance because of the presence of a phobic object), pain responses are reduced. Twenty-four spider phobics received a moderately painful electrical stimulation in each of four conditions: low anxiety/attention directed towards pain; low anxiety/attention distracted from pain; high anxiety/attention directed towards pain; high anxiety/attention distracted from pain. Anxiety was induced by means of exposure to a spider. Subjective pain ratings strongly supported the hypothesis: pain was rated lower when the $S$ diverted attention away from than when the $S$ attended to the pain stimulus, regardless of level of anxiety. The SCR to the first pain stimulus of the series of four in each condition was, however, higher when the $S$ distracted than when the $S$ attended to the pain stimulus. There were no experimental effects on later SCRs. Most importantly, there was no influence of anxiety on any of the pain responses. Attentional focus seems to be an important factor modulating the relationship between anxiety and pain.

\section{INTRODUCTION}

Despite more than 20 years of experimental work, the influence of anxiety on the experience of pain and on other pain responses is still largely unclear. Various mechanisms have been suggested. One possible mechanism is based on the analgesic effects of endorphins. It is well known that uncontrollable stress can produce endorphin-mediated analgesia (Watkins \& Mayer, 1982; Maier, Sherman, Lewis, Terman \& Liebeskind, 1983), and anxiety might also have these effects (Bolles \& Fanselow, 1980). Other physiological reactions that accompany anxiety and that can influence pain should also be mentioned. Chapman and Tumer (1986), for instance, argued that anxiety increases sympathetic activity and the release of epinephrine at the sympathetic terminals, which may sensitize or directly activate nociceptors. It has also been stated that increased arousal (which might accompany anxiety) intensifies reactions to UCSs and delays habituation (Groves \& Thompson, 1970). Finally, the gate-control theory of pain states that anxiety increases pain by opening the gate (Melzack, 1973). Thus, various physiological mechanisms have been proposed that might mediate influences of anxiety on pain. Note that the nature of the hypothesized mechanisms varies widely: some formulations imply that anxiety increases pain; others that anxiety decreases pain.

'To the present authors' knowledge nine studies have investigated the effects of experimentally induced anxiety on pain. In two of these the effectiveness of the anxiety induction was not assessed (Haslam, 1966; Bobey \& Davidson, 1970). Three studies found evidence for a pain-sensitivity-increasing effect of anxiety (Haslam, 1966; Dougher, Gold-

'Submitted for publication by Arntz, A. \& de Jong, P. 
stein \& Leight, 1987; Cornwall \& Donderi, 1988), two did not find clear effects of anxiety (Weisenberg, Aviram, Wolf \& Raphaeli, 1984; Arntz, Dreessen \& Merckelbach, 1991), and four found indications for a pain decreasing effect of anxiety (Bobey \& Davidson, 1970; Malow, 1981; Willer \& Ernst, 1986; Pitman, van der Kolk, Orr \& Greenberg, 1990). The last two studies (Willer \& Ernst, 1986; Pitman et al., 1990) demonstrated that the stress-induced analgesic effects could be reversed by an opioid blocker (naloxone), which indicates that the analgesia was endorphin-mediated. However, the Willer \& Ernst study did not include a low-anxiety condition and it is unclear whether the observed naloxone-reversible analgesia was caused by anxiety per se, or by the repeated application of very strong shocks, which constituted the stress induction. The Pitman et al. study demonstrated that Vietnam veterans with a Post Traumatic Stress Disorder (PTSD) manifested a naloxone-reversible analgesia when watching a video with Vietnam combat scenes. This important study gives rise to the question whether the analgesia was induced by anxiety, or by the (conditioned) experienced uncontrollability during actual combat. All in all, it can be concluded that empirical consensus on the influence of anxiety on pain is lacking.

Interestingly, two studies observed that the focus of anxiety influenced the results. In the Dougher et al. study, anxiety unrelated to pain had no pain sensitivity increasing effects. Only pain-related anxiety had pain sensitivity increasing effects. Some evidence for this effect was also found in the Weisenberg et al. study. However, in the Cornwall and Donderi study, pain-irrelevant anxiety also had a pain impact increasing effect. A critical analysis of previous experiments led Arntz et al. (1991.a) to hypothesize that a third, hitherto overlooked, factor modulates the relationship between anxiety and pain: attentional focus. There is clear evidence that attention to pain increases the pain experience, whereas distraction reduces it (Kanfer \& Goldfoot, 1966; Grimm \& Kanfer, 1976; Worthington, 1978; Beers \& Karoly, 1979; Tan, 1982; Turk et al., 1983; Arntz et al., 1991a). Thus, if anxiety is related to a pain-irrelevant stimulus, and this stimulus is present, it seems evident that pain responses will be reduced because attention is drawn towards the anxiety provoking stimulus. If, however, anxiety has pain as its focus, attention will probably be directed towards pain and pain responses will be increased. Therefore, attentional factors might modulate the effects of anxiety on pain to an important degree. A phobic might experience a reduction in pain during confrontation with a phobic object, whereas an hypochondriac might experience more pain when anxious about painful bodily sensations than when not anxious about them, because of attentional factors.

The Arntz et al. (1991a) study attempted to test this attention hypothesis. It was found that anxiety did not influence pain experiences and autonomic responses to a painful stimulus when attentional focus was controlled for. As hypothesized, attentional focus was more important: attention towards pain increased pain responses, whereas distraction decreased pain responses. However, the study can be criticized because of failure to induce anxiety in appr. half of the $S \mathrm{~s}$ in the high anxiety/attention condition. Nevertheless, analyses over the whole sample and over a selection of the $S s$ who met the criteria for their condition yielded identical results. Moreover, the Arntz et al. study has been criticized because two different types of anxiety were induced: anxiety related to a spider in the high anxiety/distraction condition, and anxiety related to the pain stimulus in the high anxiety/attention condition ${ }^{2}$. The present study was designed in order to overcome these problems by using the same phobic object to induce anxiety in both high anxiety conditions.

${ }^{2}$ An anonymous reviewer is acknowledged for mentioning this point. 
The aim of the present study was to investigate further the influence of anxiety on pain responses, controlling for attentional effects. A $2 \times 2$ design was employed, the two factors being anxiety (low us high) and attention (towards pain vs distracted from pain). If anxiety itself were to influence pain responses, an effect of anxiety would be found irrespective of attentional focus. If, however, influences of anxiety are for the most part mediated by attentional effects, a strong main effect of attention would be found and no, or only a slight effect of anxiety.

\section{METHOD}

\section{Subjects}

The Ss were 24 spider phobics who applied for treatment at the University spider phobia treatment project. Treatment was announced via the press and by means of a demonstration of Ost's (1989) one-session treatment in a popular television programme. All Ss were women. Their mean age was $30.3 \mathrm{yr}$ (range $15-59 \mathrm{yr}$, SD $10.3 \mathrm{yr}$ ). The mean score on the Spider Phobia Questionnaire (SPQ, Klorman, Weerts, Hastings, Melamed \& Lang, 1974) was 23.5 (SD 3.2). Subjects participated on a voluntary basis before their treatment started.

\section{Materials}

Subjective pain, subjective anxiety and amount of attention paid to the pain stimulus were rated on $100 \mathrm{~mm}$ Visual Analogue Scales (VASs). Scores were measured in $\mathrm{mm}$. Fear of spiders was assessed by means of the SPQ (Klorman et al., 1974; Frederikson, 1983). A video tape recording of an episode of 'L.A. Law' was used to distract $S$ s in the low anxiety/distraction condition. Spiders of various sizes were available in order to control the level of anxiety in both high anxiety conditions. An untransparent jar (height 137 $\mathrm{mm}$; diameter $110 \mathrm{~mm}$ ) was used in the high anxiety/attention condition so that the contained spider could not be seen by the $S$.

\section{Apparatus and physiological recording}

Apparatus was placed in an adjacent room. The pain stimulation was produced by a Siemens Neuroton 627 . The constant current stimulation was delivered to the right ankle of the $S$ via two $8 \mathrm{~mm} \mathrm{Ag-AgCl} \mathrm{Beckman} \mathrm{electrodes} \mathrm{(distance} 2.5 \mathrm{~cm}$ ). Electrodes were filled with Hewlett-Packard Redux Creme. The skin was thoroughly rubbed with Redux creme in order to reduce the electrical resistance of the skin and keep it constant (cf. Tursky, 1974). The pain stimulus had a rising time of $2 \mathrm{sec}$ to the preselected level, remained at that level for $2 \mathrm{sec}$, and was returned to zero during $2 \mathrm{sec}$. The stimulation produces a painful, stinging sensation, which is unlike electric shocks caused by electric fencing, electric mains, etc.

Skin Conductance Responses (SCRs) and Skin Conductance Level (SCL) were measured by a Beckman Conductance Coupler (type 9844), using the method of constant voltage $(0.5 \mathrm{~V})$. Electrodes $(8 \mathrm{~mm}$. Ag-AgCl Beckman) were filled with isotonic paste based on Unibase (as recommended by Fowles, Christie, Edelberg, Grings, Lykken \& Venables, 1981) and were attached to the medial phalanges of the second and third fingers 
of the non-dominant hand The first SCR starting 1-5 sec after pain stimulus onset was measured. SCRs were square root transformed (cf. Levey, 1980).

\section{Design}

A within $S$ design was employed with two within $S$ factors: anxiety (low ws high) and attertion (attention directed to the pain stimulus ws attention distracted from it). Thus, each $S$ participated in all four conditions: low anxiety/attention (LA/A); low anxiety/distraction (LA/D); high anxiety/attention (HA/A); and high anxiety/distraction (HA/D). The order of the four conditions was completely randomized across $S \mathrm{~s}$ (there are $4 !=24$ permutations). Eight variables (VAS ratings of attention, anxiety, and pain; SCL; and four SCRs) were analyzed by means of MANOVA"s with two within $S$ factors. Thus, 8 (variables) $x$ 3 (2 main factors and 1 interaction) tests were performed. A Bonferroni correction yields a level of 0.002 for each test when an overall p-level of 0.05 is employed.

\section{Procedure}

After obtaining informed consent, the $S$ filled out various rating scales and participated in a Behavioral Approach Test (BAT). Following fastening of electrodes the pain stimulation level was individually established. Stimulation level was increased in steps of $0.2 \mathrm{~mA}$ until pain threshold level was reached. A $100 \mathrm{~mm}$ VAS (not painful at all - extremely painful) was shown with at $25 \mathrm{~mm}$ an indication of the level of the pain threshold. Stimulation level was increased further in steps of $0.2 \mathrm{~mA}$. The $S$ rated subjective pain on 100 mm VASs after each step. The level was increased until a rating of more than $70 \mathrm{~mm}$ was obtained. This shock working-up procedure was performed twice in order to control for possible habituation effects. For the experiment proper, the objective level was chosen which corresponded with the first rating larger than 70 of the second working-up procedure. The mean threshold level of the second working-up procedure was $1.5 \mathrm{~mA}$, the mean level used in the experiment was $2.8 \mathrm{~mA}$. After establishing stimulation level, the first condition was introduced.

In the low anxiety/attention (LA/A) condition, the $S$ was instructed to concentrate as much as possible on the pain stimulus. It was also made clear to the $S$ that throughout the series, the stimulus would be of the same intensity and of the same duration. After instructing the $S$, the experimenter left the room and the series began after $2 \mathrm{~min}$.

In the low anxiety/distraction (LA/D) condition the $S$ was told that an episode of L.A. Law would be shown. The $S$ was instructed to concentrate as much as possible on the video and try not to let herself be distracted by the pain stimulus. It was explained to the $S$ that the pain stimulus would always remain of the same intensity and of the same duration. After instructing the $S$, the experimenter started the video and left the room. The series began 2 min later.

In the high anxiety/attention (HA/A) condition, the $S$ was instructed to concentrate as much as possible on the pain stimulus. Before starting the series, a spider was individually selected so that it made the $S$ anxious (about $60 \mathrm{~mm}$ on the VAS) when put into an untransparent jar and placed as near the $S$ as the $S$ could tolerate. The $S$ was instructed to move the jar towards herself if subjective anxiety decreased. It was also made clear to the $S$ that throughout the series, the pain stimulus would be of the same intensity and of the same duration. After placing the jar before the $S$, the experimenter left the room and the series started after 2 min. 
In the high anxiety/distraction (HA/D) condition it was explained to the $S$ that she would be confronted with a spider that would make her anxious (about 60 on the VAS) and that she would be asked to engage in various exposure exercises (such as touching the spider with a pencil, etc.). It was made clear to the $S$ that shit had complete control over what she could do and that the experimenter would not force her to do things against her will. The $S$ was instructed to concentrate as much as possible on the spider, and to disregard the pain stimuli as much as possible. It was made clear to the $S$ that throughout the series, the pain stimulus would be of the same intensity and of the same duration. After these instructions, a spider was individually selected, and the experimenter and the $S$ started with the exposure exercises. The series of pain stimuli began after 2 min.

Each condition consisted of four trials with a mean intertrial interval of $20 \mathrm{sec}$. Intertrial intervals varied randomly (range 15-25 sec). After each series of 20 trials, the $S$ was given 3 VASs (measuring subjective ratings of pain, anxiety and attention), and was left alone to fill them in.

\section{RESULTS}

\section{Effects of position of the condition}

Before the final analyses, it was checked for each dependent variable whether the position of each condition (first, second, third or fourth position in the series) influenced the results. Randomized block designs ANOVA"s with the $24 S$ s as blocks and anxiety (low ws high), attention ( $v s$ distraction), and position of the condition $(1,2,3,4)$ as factors revealed that all main and interaction effects involving position were n.s. $(p$ 's $>0.05)$, with one exception: there was a significant anxiety $x$ position interaction on the SCR of the first trial of the series of 4 trials $(F(3,57)=3.68, p=0.017)$. A Bonferroni correction (56 relevant tests are performed) would lead to the conclusion that this effect is $n . s$. , however. Moreover, the experimental effects on the first SCR were the same with or without controlling for position (controlling for position: attention: $F(1,57)=13.26, p=0.001$; anxiety: $F(1,57)=0.13$, n.s.; anxiety $\mathrm{x}$ attention interaction: $F(1,57)=0.002$, n.s.; see below for the effects without controlling for position). Therefore, the position factor was left out of consideration. A MANOVA approach was used for further analyses (that is, the four measurements of each variable were analyzed as a multivariate set, and transformed into within $S$ effects of anxiety, attention, and the interaction between these variables).

\section{Manipulation check}

As is shown in table 1 and in figure 1 , the subjective ratings of attention paid to the pain stimulus were much higher in both attention conditions than in both distraction conditions. There was a strongly significant attention effect $(F(1,23)=156,9, p<0.001)$. There was also a (much smaller) significant anxiety effect $(F(1,23)=8.14, p<0.01)$, caused by the lower attention ratings in the H.A/A condition (compared to those of the LA/A condition), and a significant interaction effect $(F(1,23)=28.5, p<0.001)$, reflecting the reduced effects of the attention/distraction manipulations in the high anxiety conditions compared to the low anxiety conditions (figure 1). 


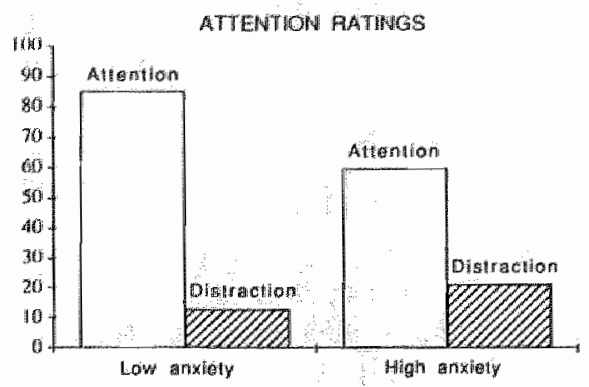

Figure 1. Mean VAS (0-100) ratings of attention paid to the pain stimulation by the $S$ in the four conditions.

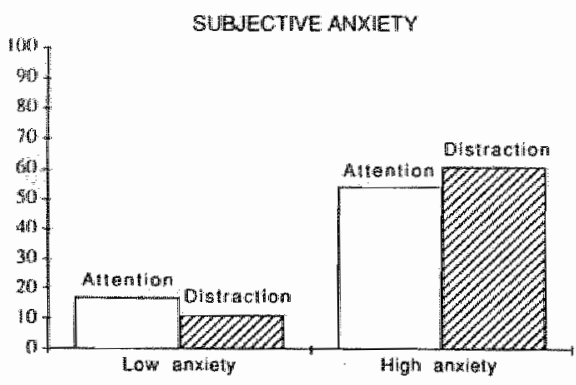

Figure 2. Mean VAS (0-100) ratings of subjective anxiety in the four conditions.

The anxiety manipulation proved to be very effective as indicated by VAS ratings of anxiety and SCL (table 1; figure $2 \& 3$ ). There were strongly significant effects of the anxiety manipulation on subjective anxiety $(F(1,23)=84.5, p<0.001)$ and on SCL $(F(1,23)=22.9, p<0.001)$.

Table 1. Means (and s.d.'s) of dependent variables in the four conditions

\begin{tabular}{|c|c|c|c|c|}
\hline \multirow[b]{2}{*}{ Variable } & \multicolumn{2}{|c|}{ Low anxiety } & \multicolumn{2}{|c|}{ High anxiety } \\
\hline & Attention & Distraction & Attention & Distraction \\
\hline Attention rating & $86(15)$ & $13(11)$ & $60(22)$ & $21(25)$ \\
\hline Subjective anxiety & $17(17)$ & $11(13)$ & $55(24)$ & $61(23)$ \\
\hline SCL (microSiemens) & $4.8(2.8)$ & $4.4(2.6)$ & $5.0(2.8)$ & $5.5(3.0)$ \\
\hline Subjective pain & $51(16)$ & $33(16)$ & $53(19)$ & $30(22)$ \\
\hline \multicolumn{5}{|l|}{ SCR (microSiemens ${ }^{3 / 2}$ ) } \\
\hline first trial & $0.62(0.43)$ & $0.77(0.38)$ & $0.63(0.39)$ & $0.79(0.41)$ \\
\hline second trial & $0.41(0.34)$ & $0.46(0.42)$ & $0.37(0.26)$ & $0.49(0.37)$ \\
\hline third trial & $0.32(0.29)$ & $0.44(0.45)$ & $0.37(0.31)$ & $0.33(0.27)$ \\
\hline fourth trial & $0.36(0.36)$ & $0.34(0.35)$ & $0.31(0.23)$ & $0.45(0.36)$ \\
\hline
\end{tabular}

The attention effects were n.s. (subjective anxiety: $F(1,23)=0.009 ;$ SCL: $F(1,23)=$ 0.09). The interaction effect was only just significant at the 0.05 level $(F(1,23)=4.39)$ in the case of subjective anxiety, and somewhat stronger in the case of SCL $(F(1,23)=7.67$, $p=0.011)$, but these effects do not reach significance if a Bonferroni corrected $p$ - level of 0.002 is employed. The interactions reflect the stronger effects of the exposure in vivo manipulation (HA/D) compared to the manipulation in the HA/A condition (spider in untransparent jar) and the anxiety/SCL reducing results of the video (LA/D) compared to non-anxious attending to the pain stimulus (LA/A); see figure $2 \& 3$ ). Nevertheless, the anxiety main effect proved to be the most important with respect to subjective anxiety and SCL.

To summarize, the experimental manipulation proved to be very effective in manipulating attention (as indicated by the attention ratings) and in inducing anxiety (as indicated by the anxiety ratings and SCL). 


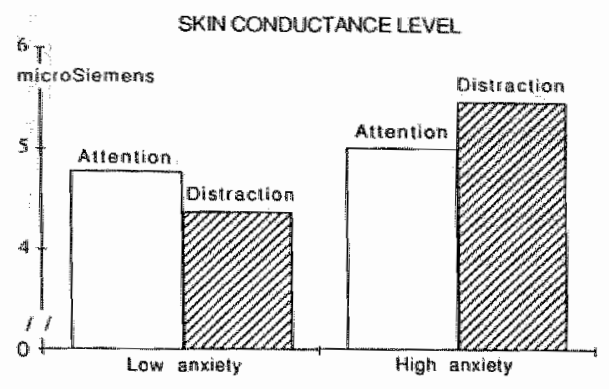

Figure 3. Mean SCL in the four conditions.

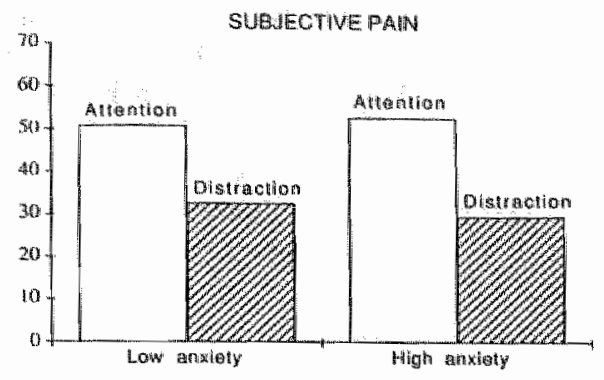

Figure 4. Mean VAS $(0-100)$ ratings of experienced pain in the four conditions.

\section{Subjective pain}

As is shown in figure 4 (and table 1), subjective pain ratings were much higher in the attention conditions than in the distraction conditions (attention effect $F(1,23)=42.1, p<$ $0.001)$. The anxiety effect was n.s. $(F(1,23)=0.02)$, as was the interaction effect $(F(1,23)$ $=0.74$ ). Thus, results strongly support the hypothesis that pain responses are influenced by focus of attention, but do not support the hypothesis that anxiety influences pain.

\section{SCRs}

Figure 5 depicts the mean SCRs to the four pain stimuli in each condition (see also table 1).

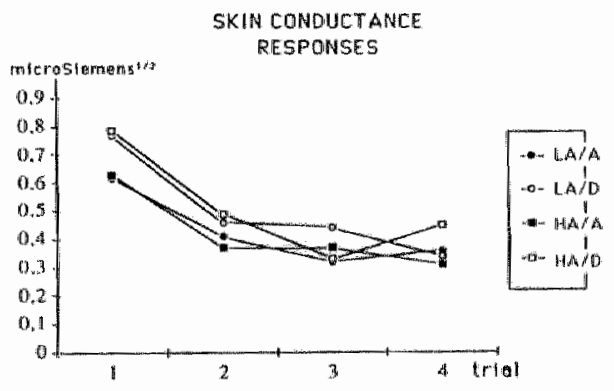

Figure 5. Mean SCRs on a trial-by-trial basis in the four conditions. (LA/A $=$ low anxiety/attention; LA/D = low anxiety/distraction; HA/A = high anxiety/attention; HA/D = high anxiety/ distraction). SCRs were transformed by square root.

There was a significant attention effect during the first trial $(F(1,23)=15.63, p=$ $0.001)$, which was much weaker during the second trial $(F(1,23)=5.87, p=0.024)$ and disappeared during the third $(F(1,23)=0.80)$ and fourth trials $(F(1,23)=2.26, p>0.10)$. Employing a Bonferroni corrected $p$-level of 0.002 leads to the conclusion that only during the first trial the attention effect was significant. The SCRs of this trial were larger in both distraction conditions compared to both attention conditions (Figure 5). Anxiety and interaction effects were n.s. for all trials $\left(p^{\prime} s>0.10\right)$, with one exception: during the fourth 
trial the mean SCR of the HA/D condition was stronger than in the other 3 conditions (Figure 5), resulting in a significant interaction effect $(F(1,23)=5.97, p=0.02)$. This effect fails to reach the Bonferroni corrected level of significance, however.

To summarize, results from SCRs do not support the hypothesis that distraction leads to weaker pain responses. On the contrary, the first SCR was clearly stronger when the $S$ diverted her attention away from the pain stimulation than when the $S$ attended to the pain stimulation. This effect was temporary, however: during later trials it disappeared. It should be noted that the anxiety manipulation did not influence SCRs to the pain stimulus at all.

\section{DISCUSSION}

The results of the present study indicate that when focus of attention is controlled for, anxiety has no, or onlly minimal influences on subjective and physiological reactions to pain. Focus of attention appears to be crucial: the experience of pain is strongly influenced by attentional focus, whether the $S$ is anxious or not. This result replicates previous findings (Arntz et al., 1991a), but now without the problems of failure to induce anxiety in a number of $S \mathrm{~s}$, with the same phobic stimulus in both high anxiety conditions, with even higher levels of subjective anxiety than in the previous study, and a more powerful within $S$ design. The pain-response-reducing effects of attention, however, were not found in the case of the SCRs. The first SCR was even larger when the $S$ distracted from the pain stimulation than when the $S$ attended to the pain stimulus. This effect might have been caused by the more surprising effect of the first pain stimulus when the $S$ is distracted compared to when the $S$ is waiting for the stimulus. It should be noted that the first stimulus was given after 2 min waiting time. In a previous study (Arntz et al., 1991a) the mean SCR of 20 trials was lower in both distraction conditions. In this study stimulation began almost immediately after the $S$ was instructed. The difference between both studies might also be attributed to the number of trials. In the present study, only four trials in each condition were given. In the Arntz et al. (1991a) study, 20 trials in each condition were given. Thus, with a larger number of trials, distraction might be related to reduced SCRs, despite a larger SCRs in the first trial compared to attention to pain.

Although the present study shows that attentional focus is an important mediating factor in the impact of anxiety on pain, it does not rule out other mediating mechanisms. That is, pain decreasing (endorphins) or pain increasing (epinephrine, arousal, etc.) factors might also play a role. It should be noted that the aim of the present paper was not to dispute the role of stress/anxiety produced endorphins and other biochemical substances that might influence pain, but to investigate another factor, the focus of attention, that might mediate effects of anxiety on pain. The present results suggest that attention is a very important factor. This conclusion is strengthened further by a more detailed comparison of the present results to those of Pitman et al. (1990). Whereas in the Pitman et al. study (also employing a within $S$ design) a pain-decreasing mean effect of the combat video of 6.6 on a 0-100 VAS was found, the present study observed a much larger effect of attention (a mean decrease of 20.7 on a 0-100 VAS). However, in the Pitman et al. study much lower pain levels (mean rating appr. 20 on the 0-100 VAS in case of the PTSD Ss) were used than in the present study (mean rating appr. 42 across all conditions), so that a floor effect may have limited the findings in the Pitman et al. study. The present study does not dispute that clear effects of stress-induced endorphins on pain responses can be demonstrated, nor that other anxiety-induced physiological factors can be demonstrated. Our data do 
suggest that attentional factors might be even more important in mediating the influence of anxiety on pain, and that attentional effects can overrule other mechanisms. This might help explain the clinical observation that in some anxious $S$ s pain experiences seem to be disproportionally elevated (despite the possible release of endorphins): anxiety about pain might lead to the direction of attention to the painful sensations.

The lack of any effect of anxiety in the present study might also be related to the pain level employed in the present study. Whereas most studies investigating stress-induced analgesia, whether using animal or human $S$ s, use pain stimuli at, or just above, pain threshold level (threshold level is often the most important dependent variable), the present study used pain levels clearly above pain threshold. Thus, it is conceivable that stressinduced analgesia can be most clearly detected at pain threshold level, but that other factors, like attention, are more important at moderate pain levels. Moreover, the type and level of anxiety might also be important. In the present study, for instance, anxiety was induced via exposure to a phobic object, which was a relatively controllable procedure for the $S$. Stimuli inducing anxiety with higher levels of experienced uncontrollability might show clearer analgesic effects. The effects of the level of anxiety should also be investigated further. It is conceivable that anxiety should surpass a threshold before it influences pain. Therefore, dose-effect investigations are needed, which document the effects of different levels of anxiety on different levels of pain, controlling for attentional effects (as in the present study) and involving a double-blind test of the effects of endorphin-receptor blockers. Such studies could also clarify whether the attentional effect observed in the present study is endorphin mediated or not (see Rose \& Orlowski, 1983, for the possible role of endorphins in the regulation of attentional focus). 


\section{CHAPTER 5. GENERAL DISCUSSION}

In this thesis various psychological influences on and psychological effects of recurrent painful experiences were investigated. In the final chapter the general discussion is organized according to these two aspects.

\section{PSYCHOLOGICAL INFLUENCES ON PAIN}

\section{Effects of incorrect pain-intensity expectations on pain}

Two forms of pain-intensity expectation should be distinguished. Firstly, "expectation" can refer to a theoretical construct, which implies that the organism is sensitive to various forms of regularity of a recurrent stimulus, forms a representation of the regular aspects of the stimulus, and reacts to deviations from this representation. One of the most clear instances of the reaction to a deviation from this type of expectation can be seen in the socalled omission response, which appears when a regularly appearing stimulus is omitted. (Sokolov, 1963; Gray, 1975). Because this type of expectation can be demonstrated in

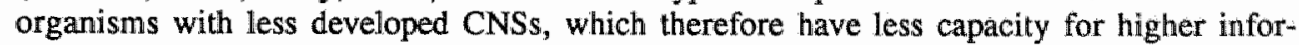
mational processes, we refer to it as taking place at 'lower" levels of the CNS. Secondly, 'expectation' can refer to the conscious anticipation of an event. This type of expectation can be symbolically expressed and can be directly influenced by various forms of information. These two forms of expectation are not necessarily perfectly associated, nor are they necessarily completely dissociated. As was concluded in section 1.2 , verbally transmitted information about a pain stimulus may, or may not, influence pain responses, depending. on the type of information (e.g. about occurrence or about pain-sensations), and depending on the pain responses (e.g. subjective experiences or physiological responses). Thus, higher informational processes may partially influence pain expectations formed at lower levels.

Effects of discrepancies between expectation and experience at the "higher' level can only be studied with careful experimental control, because otherwise the two forms of incorrect expectation are confounded. In the earlier studies on the effects of incorrect pain intensity expectations (e.g. sections $2.1,3.1-3.4$ ) these levels were not properly distinguished. Although the manipulation of the objective pain intensity level in these studies yielded experimental control over incorrect pain predictions, the 'lower' level expectation was also manipulated: as was argued in section 2.3 , the lower levels may be sensitive for sudden changes in pain intensity (even if they are correctly predicted at the higher level). Therefore the results of chapter 3 should be interpreted as related to incorrect expectations at both levels, and not only to deviations from expectations of the higher type. The results of section 2.3 can be solely ascribed to incorrect predictions at the higher level.

The effects of unpredictable increases in pain intensity on pain impact (chapter 3) can be summarized as follows. There is evidence that unsignalled sudden increases in intensity of a painful stimulus lead to a stronger immediate impact of the stimulus, both on a subjective and on a physiological level, than when the stimulus is of a constant high level of comparable intensity (section 3.4). There is also evidence that the startle response is larger with such a sudden increase (section 3.4). Furthermore, the physiological and subjective 
impact of later pain stimuli (although retumed to the previous, lower level) seems to be larger after an unsignaled increase in intensity (sections 3.11 \& 3.4). Thus, it seems that not the intensity, but the rapid unpredictable increase leads to these (dishabituation) effects. These effects may be more prominent in later phases of exposure to the stimulus than in the earlier period, probably because dishabituation is stronger the more habituation has develloped (section 3.1).

The isolated effects of incorrect pain expectations (referring to expectations the $S$ is aware of ) on the immediate pain impact divert partially from those of the unpredictable, sudden increases in pain intensity (section 2.3). Immediate physiological pain responses to the incorrectly expected pain stimullus are probably not influenced. The subjective experience may be distorted into the direction of expectation, although the results are not conclusiwe (see discussion of section 2.3). The physiological responses to later pain stimuli were found to be influenced by incorrect pain expectations, and probably more strongly by underpredictions than by overpredictions. However, subjective pain experiences were not influenced. Thus, it seems that correct conscious expectation can (partially) compensate for the negative consequences of suddenly increased pain stimuli upon later pain responses, but not for those upon the immediate impact responses. However, some remarks should be made.

Firstly, the study in which pain expectations were properly manipulated by signalling the intensity of the stimulus (section 2.3) made use of 20 stimuli of highly varying intensities. Habituation is therefore probably less than when a constant stimulus intensity is used. Thus, it is still interesting to investigate the effects of correct vs incorrect expectations about sudden increases in intensity during a series of constant stimuli (see discussion section 3.4). Such an experiment can be performed by comparing the effects of unsignalled and signalled sudden increases in pain intensity placed in a series of stimuli of constant intensity.

Secondly, in section 2.3 the assumption was made that expectations manipulated by strong external information are equiwalent to an expectation formed by the $S$ on the basis of his or her own inferences without strong extermal predictors. As was discussed in section 2.3 , strong external information might lead the $S$ to distort his or her experiences into the direction of the anchor provided by the information. In the 'real world', external information is often much more ambiguous and there are often no clear anchors. Discrepancies between expectation and experience might then result in contrast effects (which means that the painful experience is experienced as less painful the more it is overpredicted, and as more painful, the more it is underpredicted (Epstein \& Clarke, 1971; Epstein, 1971). Is this idea too far-fetched? Many people use preparatory strategies which seem to rely on a contrast effect: for instance, people often express negativistic expectations about a party, an examination, etc., and claim that 'if one expects the worst, life can bring some agreeable surprises'. ${ }^{2}$ This suggests that pessimistic expectations may have a self-serving basis. Why did we not observe such an effect? There are at least two possibilities. Firstly,

\footnotetext{
From here on, the term expectation is used as referring to expectations the $S$ is aware of; other meanings of expectation are explicitly indicated.

${ }^{2}$ This is further discussed at page 204. Moreover, the strategy might help in ambiguous domains (like the social domain), where evaluations are, to a considerable extent, constructed, but not in more unambiguous domains (such as acute, sharp pain in the laboratory, e.g. electric shock), where there is less room for construction of experiences as 'intensity'. Another possibility is, that the strategy has a social function, in preventing criticism by others after the event.
} 
people may believe in such an effect, but it may simply be non-existent. Secondly, there may be a qualitative difference between extemal information and self-inferred expectations. In cognitive therapy it has been stressed over and over again that the therapist should not argue with the patient, but facilitate the achieving of new insights by the patient on the basis of his or her own reasoning. Nisbett \& Ross (1980) demonstrated that $S$ s change their opinions radically to a new view, when they write argumentations in order to defend this new view. Debating and providing disconfirmatory information seems to have much less effect. Thus, ideas formed on the basis of own reasoning are not necessarily equivalent to ideas imposed by externall authority. These considerations are no proofs, but they lead to the possibility that incorrect pain-intensity expectations that are primarily based on the $S^{\prime}$ s own reasoning may have different effects than expectations that are based on a strong external anchor. The major research problem is, however, how to manipulate such expectations experimentally without losing experimental control over the painful stimulus. Here lies a field of research that needs a lot of development.

A related issue pertains to the subjective certainty of pain-intensity expectations. In sections 2.1 and 3.1 it was speculated that high certainty protects the $S$ against effects of relatively small deviations from expectation on pain impact, but makes the $S$ vulnerable to impact-increasing effects of large deviations (more specificaliy, to those of large underpredictions). It should be noted that this speculation is based entirely upon indirect (correlational) evidence: a third factor, for instance amount of experience with the stimulus, might cause a spurious relationship between certainty and habituation, and therefore between certainty and effects of underpredictions. Only an experimental manipulation of certainty might clarify this issue. Such a study can be done, for instance, by comparing effects of incorrect intensity signals with different degrees of certainty (e.g. ranging from pointestimates to large interval-estimates).

Returning to the observed effects of incorrect pain-intensity expectations on pain responses, the similarity with effects of unpredictable occurrences of painful experiences is striking: both do not seem to influence (long-term) subjective pain experiences, but lead to stronger physiological responding to pain. Thus, the autonomic nervous system seems to be sensitive to unpredictability, whereas the subjective judgement of the stimulus is insensitive to it. This suggests that the class of theories which assume that response-suppression develops gradually with the formation of a representation, and that a mismatch between representation and stimulus temporarily disrupts habituation, holds for autonomic responses, but cannot be applied to the subjective experience of pain ${ }^{3}$ This does not mean, however, that the parts of the CNS involved in experiences like expectations and pain sensations are completely uncoupled from the parts involved in autonomic pain responses. For the higher levels, involved in controlled information processing, seem to influence the autonomic responses to pain, as is indicated by the effects of incorrect pain expectations (of occurrence, intensity, sensations, etc.). This observation suggests that pain-responses and the experience of pain are qualitatively different (see also section 1.2).

This also implies that the A1 state in Wagner" $s$ theory is only in a metaphorical sense a short term memory and cannot be equated with the short term memory as is known from memory theoriess (Atkinson \& Shiffrin, 1968), despite Wagner"s suggestions that they are equivalent and that controlled processes may play a role (Wagner, 1978, p.180; Wagner, 1981, p.6). For if we apply the theory to controlled short term memory processes, it follows that pain responses are less the more the intensity is overpredicted. This was not observed in the experiment reported in section 2.3 . Moreover, it is unlikely that the activation of the stimulus representation into the $A 1$ state is a unitary process, because different responses generally do not wane in parallel curves. 
In the representational theories it is generally assumed that pain responses are suppressed. Thus, if the experience of pain is not a response (but more an input phenomenon), it is understandable that unpredictability does not affect it. Does this nullify the clinical imporance of correct expectations? Given that autonomic responses and general autonomic arousal may themselves lead to psychosomatic complaints, and that specific autonomic feedback loops can incrase pain complaints (Chapman \& Tumer, 1986), it seems still of clinical importance to correctly predict painful experiences.

\section{Emotions, arousal and attention}

The present thesis inwestigated two possible emotional influences on pain: those of depression/elation and those of anxiety. There was evidence that experienced pain, but not SCR, is influenced by depressed/elated mood (section 4.2). However, indirect evidence was found for the possibility that the effects of depressed/elated mood on experienced pain are mediated by another, slightly related, emotional dimension, mastery-dominance/frustration-anger. Thus, further research is indicated into the possible effects of frustrationanger on pain impact. One issue pertains to the generality of such an influence: does it only apply to situations in which the experiences of mastery-dominance us frustrationanger are directly related to the pain stimulus (such as in the study presented in section 4.2 , in which $S$ s either succeeded or failled to stop a painful stimulation); or does it apply to all sorts of angry moods, irrespective of their source? Clearly, studies in which angry mood is experimentally manipulated (perhaps by letting the $S$ observe norm-violations ?) are called for.

With respect to anxiety it was argued that attentional focus might mediate the infiuences of anxiety on pain impact (sections $4.3 \& 4.4$ ). The divergent findings of previous experiments on the influence of anxiety on pain can be explained by considering this factor. Moreover, the clinical observations that some anxious $S$ s do not seem to experience any pain when wounded, whereas other anxious $S$ s seem to suffer disproportionally, might also be explained by considering this attentional factor: when anxiety leads the $S$ to direct attention towards the anxiety-provoking stimulus (or towards flight/fight behaviour), less attention will be directed to pain and consequently the experienced pain will be increased. However, when anxiety leads the $S$ to attend to the painful sensation itself, the pain experience will probably be increased. The two experiments reported in sections 4.3 and 4.4 gave clear evidence for the mediational role of attention: anxiety, and concomitant arous-

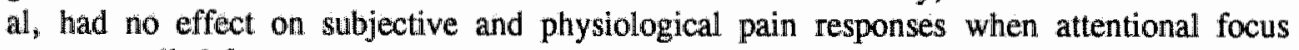
was controlled for.

These obserwations raise a number of questions. First, how is it possible that anxiety does not intensify pain and depressed mood does seem to, whereas they have the negativeevaluative aspect in common (see Tellegen, 1985; Lang, 1985)? One possibility is that moods may influence the experience of pain, whereas strong emotions do not directly intluence pain, because a strong emotion is related to an object and therefore determines the focus of attention and evaluation. As was speculated in section $4.3, \mathrm{~S}$ s in an anxious mood, for instance caused by anticipation of a feared event, might experience intensified pain when the anxiety-provoking stimulus is not immediately present. At least four possible mechanisms may play a role: (1) a possible loss of voluntary control over attention caused by anxiety, depriving the $S$ of the important pain-coping strategy of distraction from pain (see section 4.3); (2) an (even stronger) attentional effect of anxiety, leading to the automatic directing of attention towards danger-stimuli, such as pain: in the absence of other danger-cues, there may be an attentional bias towards pain (Williams et al., 1988); 
(3) a generalization of negative evaluation over all sorts of experiences; and (4) a physiological pain-intensifying mechanism evoked by the anxious mood (perhaps based on epinephrine). These mechanisms might also play a role in the influence of other negative moods on pain.

Second, how can it be explained that other studies found clear (physiologically mediated) influences of anxiety on pain? It should be noted that pain-increasing and pain-decreasing effects of anxiety have been reported and both may play a role. It is conceivable that a number of factors influence the relative strength of these effects: (1) the pain level may be important: most animal and human studies demonstrating endorphin-mediated effects of anxiety used pain threshold as dependent variable or investigated pain responses at threshold level; it is unclear whether the effects are still important for higher pain levels; (2) the level of anxiety might influence the effects: it is conceivable that physiological and attentional effects of anxiety depend on the level of anxiety - e.g. pain increasing physiological effects of anxiety (e.g. involving epinephrine) might dominate pain-decreasing physiological processes (e.g. involving endorphins) at low anxiety levels, whereas during intense anxiety their positions might be reversed; (3) the type of anxiety might be important: the possible influence of the presence $u s$ absence of the anxiety eliciting stimulus might be important (see above) because of attentional factors, and the level of experienced uncontrollability might be important: there is evidence that endorphins are especially released during high levels of experienced uncontrollability (Dantzer, 1989; Maier, 1986). Thus, there are many possibilities for further research. Nevertheless, the studies reported in the present study indicate that attentional focus is a major factor mediating the influence of anxiety on the experience of pain.

Third, the possibility should be considered that the reduced pain impact during distraction from pain is (partially) based on an oploid mechanism (see Rose \& Orlowski, 1983, for the possible role of endorphins in the regulation of attentional focus). Therefore, it would be interesting to study the effects of an endorphin blocker in studies on the influences of attention and anxiety, like those reported in sections $4.3 \& 4.4$.

Fourth, the observation that attention to pain resulted in an absence of any subjective habituation, whereas distraction resulted in steady subjective habituation, gives rise to the question whether long term habituation is similarly influenced by focus of attention (Iacono \& Lykken (1984) argue that habituation involves learning to ignore the stimulus and maintain that this can be promoted by voluntary behaviour, like distracting attention away from a stimulus). If long-term habituation is promoted by "learning to ignore" the painful stimulus, this is obviously of great clinical importance. If, however, long-term habituation is not influenced, or even hampered, by distraction from pain, the practical importance is much smaller.

\section{Perceived control}

In the theoretical section (4.1) on the effects of perceived control, a large number of laboratory studies was reviewed. It was concluded that control can, but not always does decrease pain. The effects of control vary with the type of control and the dependent variable. Positive effects are most clearly observed when the $S$ can in some way limit the painful experience (duration, intensity) by the controlling response. Pain tolerance is most clearly affected by control. Taken together, this suggests that the underlying mechanism is related to the increased certainty the $S$ gets when he or she can limit the pain. This notion is clearly related to Millers MINIMAX hypothesis (Miller, 1979; see section 4.1), but Miller could not explain why control can have a pain-decreasing effect. On the basis of 
this interpretation, it was speculated that control may have a pain-cushioning effect because the increased certainty allows the $S$ to distract his/her attention away from the painful stimulus. The present thesis does not include a direct test of this hypothesis, though the controllability study in section 4.2 gave some indirect evidence. A direct test is conceivable, by measuring attentional capacity directed to the pain stimulus via a procedure developed by Wachtel (1968) in a control and a no-control condition. Such a study would also clarify whether Miller's (1979) opposite theory is right (that with control, Ss attend more to the painful stimulus than without control), although the possibility should be considered that this may depend on the exact nature of the controlling response, which may require attention to the painful sensation in different degrees.

A direct test of the control-certainty-distraction hypothesis is the more so indicated, as we are confronted with contradictory effects of two uncertainty-increasing experimental procedures. Whereas (un)controllability and incorrect predictions both affected certainty and physiological pain responses, uncontrollability also influenced the experience of pain, but incorrect predictions did not (sections $2.3 \& 4.2$ ). If uncertainty about how much pain is to be expected leads to increased (involuntary) attention to pain, then both types of uncertainty-increasing experiences should have resulted in an increased experience of pain. At least two explanations can be offered.

First, although both procedures affect the ratings of certainty about pain-intensity predictions, the underlying variable may be qualitatively different. As was argued by Miller (1979), the possibility of exercising control over an aversive stimulus places the source of certainty within the $S$ : thus, the $S$ can limit the aversiveness of the event by his or her own action. In contrast, certainty that is based upon correct predictions leaves the $S$ at the mercy of external forces. Thus, it may be hypothesized that (automatic) reduction of attentionall capacity allocated to an aversive stimulus is faster with an internal source of certainty that the stimulus will remain below tolerable levels.

Second, certainty about how much pain to expect may affect physiological pain responses (SCRs), and anticipatory fear, but not the experience of pain. This explanation (correctly) predicts that physiological responding is influenced by both types of experimental procedures, but it cannot explain why controllability affects the experience of pain. Perhaps the influence of controllability on the experience of pain is based on another mechanism. In fact, in section 4.2 evidence was found for the notion that the effects of controllability on SCRs are mediated by certainty, whereas the effects on the experience of pain seemed to be mediated by an emotional variable. Thus, the influences of controllability on pain responses may not be based on a unitary process involving certainty.

Two other underlying mechanisms were suggested in section 4.1 . One states that a painful experience gets a different meaning if it can be controlled. The $S$ may reason as if controllable pain is not based on a harmful process and uncontrollable pain is. The resulting different meanings might influence the experienced pain level and physiological responses. However, it is not clear how this theory can explain all laboratory findings, which generally employ externally applied harmless stimuli. The other suggested mechanism is that acquiring and exercising control is a positive experience per se, and failing to exercise control (and losing control) is a negative experience. In section 4.2 an attempt was made to investigate whether the presumed positive/negative experiences of (no) con- 
trol are related to elated/depressive mood."

In this study it was concluded that elated/depressed mood and control seem to have independent, additive influences on expenenced pain. Thus, there was little evidence that the pain-decreasing effects of control were mediated by increased mood on the elation/depression dimension. However, there were indications that another emotional dimension is essential: anger seemed to mediate the influences of both the elated/depressed mood and control on experienced pain. Thus, controllability might be closely related to a specific emotional dimension of dominance-mastery/frustration-anger (Lang, 1985; Osgood, 1962). One obvious test of this interpretation, the investigation of effects of induced angry mood on pain, was already suggested. Another implication is that $S s$ who refrain from trying to acquire control over uncontrollable pain should suffer less from pain, because they should feel less frustrated and angry. In section 4.1 it was theorized that chronic pain patients who try to acquire total control over their pain (total relief) may suffer disproportionally because of the negative effects of their failure, whereas chronic pain patients who accept their pain and try to find control over realizable aspects might suffer less (see also Silver et al., 1982; Rothbaum et al., 1982). Clinical observations give indirect evidence for this view, and also suggest that anger might play a mediating role. Clearly, prospective research is indicated.

Some other possibilities for future research on clinical aspects of controllability should be mentioned. First, the view that chronic low back pain patients suffer from a specific form of learned helplessness deserves further tests. Field studies of high methodological quality seem to support this theory (e.g. Ruby et al., 1988), but experimental tests would give further evidence. In a laboratory study Sandra Gransier and I (1991) tried to test the hypothesis that CLBP patients profit less from exercising control over laboratory pain than normal controls, because learned helplessness related to chronic pain would imply that the $S$ has difficulties in learning/experiencing new control possibilities. The results supported the hypothesis: whereas normals who exercised control over pain experienced less pain and reacted physiologically less to the pain stimulus than normals who could not exercise control, CLBP patients did not profit from exercising control. However, to the best of the present author's knowledge, this has been the only direct experimental test so far. Therefore, more tests are needed.

With respect to acute clinical pain, research on controllability (for instance in the dental treatment setting) has used controllability manipulations that seem to be much less than optimal (see section 4.1). More powerful ways of giving the patient control should be investigated (for instance giving the patient control over the power of the drilling apparatus).

This study also investigated whether induced mood influenced perceptions of control and, via these perceptions, pain responses. Little evidence was found for the theory that mood influences pain by influencing perceived control, although depressed mood retarded perceiving control when control was exercised. However, a number of methodological problems should be mentioned: (1) possibly, a floor effect obscured mood effects in the no-control conditions; (2) the way perceived control was measured may have been inadequate, stressing the correctness of the response more than the experienced sense of control over pain. On the other hand, ratings of perceived control over pain intensity may become redundant, if the $S$ bases this rating only on the experienced pain reduction. 
Most of the research on psychological influences on pain has been directed to factors that may decrease pain responses. The major clinical problem, however, is how pain experiences that are disproportionally elevated compared to their (possibly) physiological basis, or have no physiological basis at all, can be understood. Although it has been argued in section 1.2 that it is unlikely that the experience of pain can be classically conditioned, research into the possibility that evaluative conditioning (Martin \& Levey, 1987) can change the evaluative aspects of the pain experience might lead to interesting new insights and treatment possibilities. Of course, this possibility is highly speculative. Another potentially fruitful research area pertains to the influence of worrying or other forms of negativistic mental activities, that are not corrected by external information, on the experience of pain. Recent developments indicate that human $S$ s can increase their (neurotic) responses to conditioned and unconditioned stimuli by engaging in rehearsal of the UCS (Jones \& Davey, 1990; van den Berg, 1989), and that worrying can have an incubation effect (Borkovec, 1985; York et al., 1987). It is conceivable that pain experiences can be increased by such processes (for instance, a chronic pain patient may, as soon as he or she feels some pain, engage in negative ideation about the injustice that has been done to him or her during or after the incident that led to the initial pain complaints, thereby amplifying immediate and later pain experiences). Finally, it has often been suggested that the meaning of the pain influences the way it is experienced (section 4.1). However, this notion is mainly based on clinical observations and needs further empirical study. Even if these prospects shed new light on pain problems, the issue of whether pain experiences can be produced purely by psychological processes (without any neuronal transmission of pain-signals) remains unresolved. On the basis of present scientific knowledge, the claim of some clinicians that this is possible seems unjustified.

\section{PSYCHOLOGICAL EFFECTS OF PAINFUL EXPERTENCES}

The effects of two kinds of painful experiences were studied. Firstly, the effects of incorrectly expected pain; and secondly, the effects of pain which varies unpredictably in intensity.

\section{Pain expectatilons}

In this thesis various tests with increasingly stringent methods were performed to test the notion that people immediately correct disconfurmed pain predictions. More specifically, it was argued that the observed changes in prediction level after spontaneous mismatches can to a large degree be explained by random processes (see section 2.2). Nevertheless, statistical procedures controlling for these processes still indicated that people do show 'real' changes in pain predictions. However, this does not necessarily increase our understanding of clinical problems: people probabily correct predictions of all sorts of events in a similar way, and they probably show the same sort of recency effect as was observed in section 2.1 (in that the previous experience strongly influences the next prediction, at least when there is a short interval between the two instances). What is clinically more interesting, are (1) the effects of incorrect pain predictions on the acquisition of fear (on the subjective, physiological and behavioural level); (2) the asymmetrical deviati- 
ons from this simple model; and (3) the relationships between pain expectations, fear and avoidance behaviour.

\section{Acquilred fear}

Fear acquired by experiences with painful events is not only related to the intensity of the pain, but also to its predictability. In the present thesis an altempt was made to test the influences of unsignalled sudden increases in pain intensity and of incorrect pain expectation on several aspects of acquired fear: subjective fear, anticipated pain (pain expectation), physiological anticipatory reactions, escape and avoidance behaviour. The results indicate that unsignalled sudden increases in pain-intensity contribute to all these fear parameters. Incorrect pain predictions, more preciselly underpredictions and not overpredictions, were also found to be related to fear, but here a smaller number of fear-parameters was tested. Originally, the sudden increases in pain intensity were designed to induce underpredictions, but, as was argued before (e.g. section 2.3), this procedure confounds sudden increases per se with underpredictions. Nevertheless, the results of the unconfounded underprediction induction procedure (section 2.3) are strikingly similar to those of the intensity-increase procedures used in chapter 3 . What is the relative importance of these two types of fear induction? Can the effects of unsignalled, sudden increases in pain be ascribed to the underprediction of the $S$ ? If we assume that the fear responses are primarily based upon expectation, we might expect that this is true. However, it is conceivable that the sudden increases themselves lead to autonomic fear responses that can only partially be influenced by knowing the intensity of each experience in advance. Our experiments cannot resolve this issue. A direct investigation of the relative effects of these two factors is therefore indicated.

Such an experiment might also clarify a related issue, namely the development of defensive $v s$ orienting anticipatory reactions. In section 3.4 the influence of unpredictability of pain-intensity on the kind of physiological anticipation (defensive ws orienting; or, in another interpretation, activation for flight/fight ws 'acceptance'; as indicated by the direction of heart rate changes, acceleration $v s$ deceleration) was investigated. Unsignalled, sudden increases to a high pain level were found to lead to anticipatory heart acceleration, whereas a constant (therefore predictably) high level of pain was anticipated with heart rate deceleration. It was speculated that unpredictable UCS intensity might be related to the development of a 'phobic' type of fear. It would not only be interesting to replicate this finding, but also to unravel the contributions of incorrect expectations and the rapid intensity increase per se. ${ }^{5}$

In sections 2.1 and 3.1 correlational observations led to the speculation that high certainty about expected pain-intensity would reduce the effects of small discrepancies between expectation and experience, but would be related to more disrupting effects of large discrepancies (underpredictions). Although no experimental test was done, a generalization of the finding that underpredicted painful events contribute to acquired fear, support the second part of this hypothesis: if certainty is low, the $S$ bears in mind that the event will fall in a wider intensity range than when certainty is high. Therefore, the 'size' of underprediction is less in the first case than in the second case, in other words: the loss of predictability and the breakdown of the predictive schema are less. Still, it would be

In the experiment reported in section 2.3 heart rate was unfortunately not measured, because of resource limitations. 
interesting to test this supposition directly by experimentally manipulating the range of expectation. This would also produce the opportunity to investigate the hypothesized interaction between certainty and size of discrepancy.

\section{Asymmetrical effects}

Although immediate correction of expectation after an incorrectly predicted experience seems the rule, a striking asymmetry was observed: whereas underpredictions generally lead to an immediate increase in expected pain approx. to the level of the last experience, overpredictions are followed by more moderate corrections. More disconfirmations are needed before the $S$ lowers his or her prediction to the level of the last experience, whereas one disconfirmatory underprediction is enough to increase the prediction level. A similar pattern is shown by the certainty the $S$ s have about their expectations. The experience of an underprediction leads to protracted uncertainty, whereas overprediction does not disturb the certainty the $S$ s experience about their expectations (although there may be some temporary uncertainty after an overprediction, this is not long-lasting). ${ }^{6}$

How can this asymmetry be explained? Several explanations have been suggested (see for instance Rachman \& Arntz, 1991). Initially it was suggested that underpredicted painful experiences hurt more than correctly and overpredicted experiences. If this is true, overprediction and a reluctance to lower increased predictions after an underprediction might be understood as a "voluntary" attempt of the $S$ to protect him or herself against the alleged negative effects of an underprediction. However, a direct test of this supposition did not give any evidence for it at all (see however Epstein \& Clarke, 1970, for positive evidence), although it might be argued that avoidance of underprediction has a functional value because underpredictions lead to autonomic arousal (see sections $2.3 \& 5.1$ for a more extensive discussion). If overprediction is not a sort of 'voluntary' covert behaviour, it leads to another interpretation of the explanation people often give about their pessimistic predictions (see the previous section): perhaps the explanation that a pessimistic strategy helps reducing the effects of aversive events is a rationalization, an attribution about an experience (namely that they have involuntary pessimistic expectations) people find otherwise difficult to explain and they cannot change. They may even believe in it, although it does not work!

Several other explanations for the asymmetry can be offered. First, cognitive research has demonstrated that people have more difficulties in processing "non-events" than events, and that "vivid and salient events' exert a disproportional influence in judgments under uncertainty (Alloy \& Tabachnik, 1984; Nisbett \& Ross, 1975; Tversky \& Kahneman, 1974). These stimulus properties may influence the immediate processing of the event and/or to the retrievability of the event in memory (Wardle, 1984). Thus, it can be deduced that a painful experience will have an impact on later predictions (and probably on memories) that depends on its intensity. But this does not explain the effects of the discrepancy between expectation and experience. If we are to explain the effects of discrepancies in this theoretical framework, it must be assumed that the saliency of painful experience, at least with respect to expectations, fear and memories, is not only a function of its intensity, but also of the discrepancy between expectation and experience. In other

\footnotetext{
${ }^{6}$ The ratings of certainty were interpreted as indications of the range in which the $S$ expects the intensity of the next experience will fall. It would be interesting to investigate this more directly by asking $S s$ to make interval estimations, or even (computerized) chance distributions.
} 
words, the saliency of the painful experience is partly determined on the basis of the direction and size of discrepancy with expectation.

A second explanation is based on the observation in our study with dental patients (section 2.4) that experienced anxiety during a disconfirmatory experience reduced the disconfirmatory effect of it on later expectation level and fear. Three underlying processes were suggested: (1) the experience of anxiety may be an aversive experience per se, which reinforces fear and pessimistic expectations (such processes have been proposed by Beck et al., 1985, and Eysenck, 1986); (2) a state of anxiety may disrupt information processing, so that reality testing is hampered (e.g., Wachtel, 1968); and (3) at least some (neurotic?) $S$ s may have difficulties distinguishing real danger and the subjective experience of anxiety.

Lastly, it can be maintained that the asymmetry has a functional basis, not with respect to the impact of the painful event, but with respect to preparation for fight/flight and avoidance behaviour. Why does the loss of predictability the organism is confronted with after a mismatch have different effects depending on the direction of the discrepancy between expectation and experience? An underprediction might lead to fear and caution because certainty is lost that the event will not exceed a tolerable level ('if it exceeds the expected level unpredictably, it might as well become unpredictably intolerable or lifethreatening'). An overpredicted event, on the other hand, means loss of certainty with respect to a less dangerous direction, obviously less important information for survival. It should be noted that this functionality interpretation does not imply teleology: as with habituation, classical conditioning, and other forms of learning, mechanical (causality) models and functionality analyses can both be made. Thus, the 'conservative bias' after an underpredicted painful experience might help the organism to increase survival chances by promoting caution, preparation for flight/fight and avoidance.

\section{Pain expectations, fear and avoidance/escape behaviour}

The tendency to chronically overpredict the aversive consequences of events seems to be a characteristic of neurotic problems (e.g. section 2.4). In other words, overprediction might be an important cognitive component of it, and is probably related to avoidance behaviour. However, it seems that not only the expected intensity of the aversive event, but also its predictability, notably whether it unpredictably exceeds expectation or not, is related to fear, and therefore to escape and avoidance. In two experiments an attempt was made to test the hypothesis that underpredicted painful experiences lead to escape and avoidance behaviour (sections $3.2 \& 3.3$ ). However, because of the employed experimental manipulation we cannot be sure that the discrepancy between expectation and experience is causally related to the observed escape and avoidance behaviours, or that the sudden increase was the essential factor (see sections 2.3 and 5.1 for a discussion of this issue). Nevertheless, the final test of the influences of incorrect pain expectations gave clear evidence that they affected subsequent pain expectations, certainty, and fear (section 2.3). Assuming that voluntary behaviour, including avoidance/escape, is at least partially based on the subject"s considerations about the expected consequences of the event, it follows that the discrepancy between consciously expected and experienced events pllays a causal 
role in awoidance/escape behawiour. ${ }^{7}$ Some interesting new research issues follow. For example, the possibility tha: some sort of (empathetic) confrontation of the discrepancy between expected and experienced aversiveness may help $S$ s to reduce overprediction, fear and avoidance behaviour (Rachman \& Amtz, 1991). More specifically, is the reduction of these variables only dependent on formal characteristics of disconfirmatory experiences (such as number of, intervals between, and length of the experience ${ }^{8}$ ), or can the effects be increased by promoting the processing of each event (e.g. by repeated re-processing of the discrepancy between expectation and experience; by construing a new experience as an explicit test of the expectation and optimalizing informational processing during the experience)? A second research issue is the investigation of separate clinical problems on which kind of overprediction plays a major rolle in maintaining the problem. For example, are panic patients characterized more by an overestimation of the chance of a fatal effect of a panic attack, or by an overestimation of the amount of experienced anxiety during an attack? Are CLBP patients who avoid physical activities characterized by expected immediate or long-term pain?

\section{Some more new research possibilities}

The asymmetry between the effects of both types of incorrect pain predictions gives rise to the question whether there is also such an asymmetrical effect on long-term memories of pain. In other words, are underpredicted painful experiences more memorizable than overpredicted experiences of equal intensity? If the present interpretation of the asymmetry is correct, this might be expected, because of the importance of the information of an underprediction, and its breakdown effects on the $S$ 's schema. Experimental tests of this hypothesis are clearly indicated. A related issue pertains to the relative importance of early ws late intensely painful events in a series of moderately or non-painful experiences on the acquisition of fear. Learning theory predicts that late intensely painful experiences should have less effect on the acquisition of fear, because of latent inhibition. The present findings suggest however that a late intensely painful experience will also lead to fear, if it occurs umpredictably, because of the associated alarming loss of predictability. In fact, Davey (1989) found in a retrospective study on dental fear that both early and later very painful experiences are related to dental fear. In other words, intense UCSs later in a series might nullify the effects of latent inhibition. Davey did not discuss the contribution of the incorrect expectation the $S$ probably had to the acquisition of fear, but our findings suggest that this may be essential for the fear-evoking effects of later intense UCSs. Finally, a potentially fruitful research area has already been suggested in the context of pain-increasing factors: the effects of worrying, catastrophic fantasizing, and other types of reprocessing of painful experiences, like neutralizing, suppression, etc., on the

\footnotetext{
The observation that (phobic) $S s$ engage in approach behaviour after receiving information that influences expectation is also an indication that conscious expectations play an important rolle in voluntary behaviour (e.g. dental phobics who apply for 'pain-free' treatment; agoraphobics doing exposure exercises on the basis of a rationale; pain patients doing physiotherapeutic exercises they formerly feared because of expected increase in pain).

The findings in section 2.4 indicate that the interval between disconfirmatory experiences should not be too long (better weeks than months), and that the disconfirmations should continue until a correct prediction level is reached, otherwise memories of the event and new predictions will (partially) return to the original, high level.
} 
development of anticipatory variables deserve further study. This is a relatively new area of experimental research, possibly because it is difficult to exert reliably experimental control over such processes. But, these processes seem to be very much related to processes that are often observed clinically, and investigating them seems to be essential for the development of valid cognitive theories of the acquisition of disproportionally fearful and pessimistic anticipation of painful events. Insight in these processes might be especially important for the understanding of human anticipation of pain. 


\section{SUMMARY}

This thesis deals with some psychological effects of and psychological influences on pain. More specifically, the influences of emotions, attentional focus, incorrect predictions of pain, unpredictable increases in pain intensity, and controllability are investigated.

Chapter 1 offers an introduction to psychological aspects of pain, resulting in the selection of research issues. It is argued that pain is a multidimensionally psychological phenomenon. The following potential influences on pain are discussed: arousal, emotions, attention, operant conditioning, classical conditioning, predictability of occurrence, certainty, pain-intensity expectations, and controllability. Of the effects of pain, pain expectations, memories of pain, emotional after-effects (including anticipatory fear), and learned helplessness are treated. Finally, the chapter presents an overview of research questions.

Chapter 2 presents four studies on the interrelationships between experiences of pain, expectations of pain, memories of pain, and fear of pain. The studies concentrate on the effects of incorrect predictions of the intensity of a painful experience. Some hypotheses of Rachman's match/mismatch model are put to the test with increasingly stringent methods, methodological criticism on previous studies is discussed, and new hypotheses are formulated. Special attention is paid to the asymmetrical effects of under- vs over-predicted painful experiences: underpredictions, but not overpredictions, appear to cause fear, the tendency to overpredict new experiences, uncertainty, and stronger physiological responding to pain. One hypothesis, that overprediction results from avoidance of under-predicting pain because underpredicted pain hurts more, is refuted. Information-processing ('null effects are more difficult to detect than positive effects') and functional ('the alarming effects of an underprediction leads to caution and prepares for flight/fight or avoidance behaviour, which might increase survival chances') explanations are discussed. Finally, incorrect pain predictions are studied in the clinical context of dental treatment. In agreement with the hypotheses, fearful patients tend to overpredict pain, slowly reduce their prediction levels to more correct levels, and show a partial return to the initial prediction level at a 5-months follow-up. Their memories of pain also appear to be biased. Change processes are explored, and the observation that a state of anxiety interferes with the effects of disconfirmations is discussed.

Chapter 3 presents studies on the effects of unpredictable increases in pain intensity. These appear to cause temporary dishabituation of subjective and physiological responding to pain, and to contribute to subjective fear and physiological anticipation reactions, as well as to escape from and avoidance of the pain stimulus. There are indications that not so much the intensity of the pain stimulus as the unpredictability of its intensity causes these effects. It is speculated that unpredictability of the intensity of a painful stimulus contributes to the acquisition of phobic fear of the aversive stimulus, whereas predictability of intensity is related to a more neutral anticipation of the aversive event.

Chapter 4 starts with a theoretical section on controllability and pain. First, laboratory studies are reviewed and it is concluded that those forms of control by which the $S$ can limit the pain, so that the pain cannot become of intolerable duration or intensity, have the clearest positive effects. Second, studies on the effects of controllability on acute pain in two clinical contexts, dental treatment and childbirth, are reviewed. It is concluded that there are clear indications that control can reduce the experience of acute clinical pain. Third, the role of lack of perceived control over pain in chronic pain problems is discussed. Two major issues are treated: whether learned helplessness theory can increase our insight in chronic pain problems, and the role of experiencing control in the successful 
treatment of chronic pain problems. Finally, possible processes underlying the pain-decreasing effects of control are discussed.

The next sections present laboratory studies on the influences of control, attentional focus, elated/depressed mood, and anxiety on pain. The possible influences of elated/depressed mood on the effects of controllability are investigated, with special reference to two theoretical notions. First, that mood influences effects of control via influencing perceptions of control. Second, that the effects of controllability are mediated by elated/depressed mood. The findings give rise to the speculation that not elated/depressed mood, but another emotional dimension, dominance-mastery/frustration-anger, mediates the influence of controllability on pain. Finally, the theory that attentional focus mediates the influence of anxiety on pain is put to the test in two experiments. This theory might explain the lack of convergence of previous empirical studies, and the contradictory clinical observations. Subjective pain reports give strong evidence for this theory, but physiological responses yield more equivocal results.

Chapter 5 is devoted to a general discussion of the major findings of the studies of the present thesis. It is argued that two forms of pain expectations can be distinguished, one form referring to a theoretical construct, and one form referring to a variable more directly observable by reports of the $S$. Painful experiences exceeding both types of pain-intensity expectations appear to contribute to the development of anticipatory fear and to dishabituation on the physiological level. Our observation that incorrect pain-intensity predictions do not influence immediate pain reactions is further discussed in relationship with the degree of ambiguity of external sources of information about pain intensity and the often observed explanation people offer for their pessimistic expectations. The observed emotional and attentional influences on pain reactions give rise to a number of new research issues, such as a direct experimental test on the possible influence of angry mood on pain, the effects of different levels and types of anxiety on different levels of pain-intensity, the role of endorphins in attentional influences on pain, and an investigation into the effects of attentional focus on long-term habituation. With respect to controllability, it is stressed that the certainty-distraction hypothesis of the positive effects of control should be studied more directly, the more so as other certainty influencing manipulations do not seem to influence the experience of pain. The failure of psychological theories to clearly explain how the experience of paim can be disproportionally elevated, or even exist without any neurological pain-signal, is discussed and some new research possibilities are suggested.

With respect to the development of pain-anticipation reactions, the relative contributions of the two types of expectations are discussed. Experiences exceeding both types of experiences seem to contribute to the acquistion of fear, the tendency to overpredict pain, and uncertainty about pain predictions. A direct test comparing the contributions of incorrect pain expectations of the second form with the effects of rapid (but predictable) intensity increases per se might be interesting, especially with respect to the development of phobic-like anticipatory reactions (heart rate acceleration and avoidance). Possible explanations for the asymmetrical effects of both types of incorrect pain predictions (under- and overpredictions) are discussed. The role of overpredictions and uncertainty, pertaining to the lack of predictabillity of intensity of aversive events, in fear and avoidance is treated. New research is suggested with respect to the influences of incorrectly predicted painful experiences on memory bias (are underpredicted painful experiences more memorable than correctly predicted experiences?) and on the disruption of latent inhibition. Finally, it is speculated that research into specific ways of reprocessing painful experiences might increase our insight into human anticipation of pain. 


\section{SAMENVATTING}

In dit proefschrift worden enkele psychologische effecten van en psychologische invloeden op pijn behandeld. Centraal in het onderzoek staan de invloeden van emoties, de focus van de aandacht, onjuiste pijn voorspellingen, onvoorspelbare verhogingen in pijn intensiteit, en controleerbaarheid.

Hoofdstuk 1 biedt een inleiding tot de psychologische aspecten van pijn, hetgeen resulteert in een selectie van de onderzoeksthema's. Het wordt beargumenteerd dat pijn een multidimensioneel psychologisch fenomeen is. De volgende potentiele invloeden op pijn worden bediscussieerd: arousal, emoties, aandacht, operante conditionering, klassieke conditionering, voorspelbaarheid van optreden van pijn, zekerheid, pijn-intensiteits verwachtingen, en controleerbaarheid. Wat betreft de gevolgen van pijn worden pijn verwachtingen, herinneringen aan pijn, emotionele na-effecten (waaronder anticipatie angst), en aangeleerde hulpeloosheid besproken. Het hoofdstuk eindigt met een overzicht van de onderzoekswragen.

In hoofdstuk 2 worden vier studies naar de onderlinge relaties tussen pijnlijke ervaringen, pijn verwachtingen, herinneringen aan pijn, en angst voor pijn gepresenteerd. Deze onderzoeken concentreren zich op de effecten van onjuiste voorspellingen van de intensiteit van een pijnlijke ervaring. Enkele hypothesen van Rachman's match/mismatch model worden getoetst met toenemend striktere methoden, methodologische kritiek op eerdere studies wordt besproken, en nieuwe hypothesen worden geformuleerd. De aandacht gaat speciaal uit naar de asymmetrische effecten van onder- versus overschatte pijnlijke ervaringen: onderschattingen, maar niet overschattingen, blijken te leiden tot angst, tot de geneigdheid om nieuwe ervaringen te overschatten, tot onzekerheid, en tot sterkere fysiologische reacties op pijn. Eén hypothese, dat de geneigdheid tot overschatten ontstaat door het vermijden van onderschattingen, omdat onderschatte pijn pijnlijker zou zijn, wordt verworpen. Verklaringen gebaseerd op informatieverwerkingstheorie ('de afwezigheid van een gebeurtenis is moeilijker te detecteren dan de aanwezigheid van een gebeurtenis') en op functionaliteit ('de alarmerende effecten van een onderschatting leiden tot voorzichtigheid en prepareren het organisme voor vlucht, vecht of vermijdings gedrag, hetgeen de overlevings kansen kan vergroten') worden besproken. Tenslotte worden onjuiste pijn verwachtingen bestudeerd in de klinische context van de behandeling bij de tandarts. In overeenstemming met de hypothesen overschatten angstige patiënten de pijnlijkheid van de behandeling, stellen zij hun onjuiste pijn verwachtingen slechts langzaam bij, en vertonen zij een gedeeltelijke terugkeer naar het oorspronkelijke hoge pijn verwachtingsniveau bij een follow-up meting 5 maanden later. Hun herinneringen aan de pijnlijkheid van de behandeling blijken eveneens vertekend te zijn. Veranderings-processen worden geëxploreerd, en de bevinding dat een angstige toestand tijdens de behandeling interfereert met de effecten van disconfirmerende ervaringen wordt besproken.

In hoofdstuk 3 worden experimentele studies naar de effecten van onvoorspelbare verhogingen in pijn intensiteit gepresenteerd. Deze blijken een tijdelijke dishabituatie van subjectieve en fysiologische pijn responsen te veroorzaken, bij te dragen tot subjectieve vrees en fysiologische anticipatie reacties, en te leiden tot ontsnappings- en vermijdingsgedrag. Niet zozeer de intensiteit van de pijnlijke stimulus, als de onvoorspelbaarheid van de intensiteit lijkt deze effecten te veroorzaken. Er wordt over gespeculeerd dat de onvoorspelbaarheid van de intensiteit van een pijnlijke stimulus bijdraagt aan het ontstaan van fobische angst voor de aversieve stimulus, terwijl voorspelbaarheid van intensiteit gerelateerd is aan een meer neutrale anticipatie van de aversieve gebeurtenis. 
Hoofdstuk 4 begint met een theoretische sectie over controleerbaarheid en pijn. In de cerste plaats worden laboratorium studies besproken en wordt geconcludeerd dat die vormen varn controleerbaarheid waarmee het subject de pijn kan begrenzen, zodat de pijn niet onverdraagligk sterk of langdurig wordt, de duidelijkste positieve effecten hebben. In de tweede plaats worden onderzoeken naar de effecten van controleerbaarheid op acute pijn in twee klinische situaties, de behandeling bij de tandarts en bevallingen, besproken. De conclusie luidt dat er duidelijke aanwijzingen zijn dat het uitoefenen van controle de ervaring van acute klinische pijn kan verminderen. In de derde plaats wordt de rol van gebrek aan ervaren controle over pijn bij chronische pijn problemen besproken. Twee hoofdzaken worden behandeld: de vraag of de aangeleerde hulpeloosheids theorie ons inzicht in chronische pijn problematiek kan vergroten, en de rol die het ervaren van controle speelt in de succesvolle behandeling wan chronische pijnproblemen. Tenslotte worden mogelijke processen die aan de pijn-verminderende effecten van controle ten grondslag liggen bediscussieerd.

In de volgende secties worden laboratorium experimenten naar de invloeden van controle, de focus van de aandacht, opgewekte/depressieve stemming, en angst op pijn gepresenteerd. De mogelijke invloeden van opgewekte/depressieve stemming op de effecten van controleerbaarheid worden onderzocht, waarbij de aandacht in het bijzonder uitgaat naar twee theoretische mogelijkheden. In de eerste plaats zou stemming de effecten van controleerbaarheid kunnen beinvloeden door de perceptie van controle te kleuren. In de tweede plaats zouden de effecten van controleerbaarheid gemedieerd kunnen worden door opgewekte/depressieve stemming. De bevindingen geven aanleiding tot de speculatie dat niet opgewekte/depressieve stemming, maar een andere emotionele dimensie, dominantie-overmacht/frustratie-woede de invloeden van controleerbaarheid op pijn medieert. Tenslotte wordt de theorie dat de focus van de aandacht de invloed van angst op pijn medieert getoetst in een tweetal experimenten. Deze theorie zou in staat kunnen zijn om het gebrek aan overeenstemming tussen eerdere experimenten, alsmede de elkaar tegensprekende klinische observaties, te verklaren. De theorie wordt sterk ondersteund door de uitkomsten op het gebied van subjectieve pijn ervaringen, maar de fysiologische responsen blijken een minder eenduidig beeld te geven.

Hoofdstuk 5 is gewijd aan een algemene bespreking van de belangrijkste bevindingen van de onderzoeken die in dit proefschrift zijn opgenomen. Het wordt beargumenteerd dat twee soorten van pijn verwachtingen onderscheiden kunnen worden, waarvan eén verwijst naar een theoretisch construct, de ander naar een variabele die meer direct observeerbaar is door rapportage van het subject. Bij beide soorten pijn verwachtingen blijkt dat, wanneer de pijn ervaring de verwachting overstijgt, anticipatie angst langdurig toeneemt en dishabituatie van fysiologische pijnresponsen optreedt. De observatie dat onjuiste pijnintensiteits verwachtingen de onmiddellijke pijn reacties niet beïnvloeden wordt besproken in relatie tot de mate van ambiguitteit van externe bronnen van informatie over pijn intensiteit en de vaak gehoorde verklaring die mensen geven over hun pessimistische verwachtingen. De gevonden invloeden van emoties en aandacht op pijn leiden tot een aantal nieuwe mogelijkheden tot verder onderzoek, zoals een directe experimentele test van de mogelijke invloed van een geërgerde stemming op pijn, de invloeden van verschillende niveaus en soorten van angst op verschillende intensiteiten van pijn, de rol van endorfinen in de aandachts invloeden op pijn, en een bestudering van de effecten van de focus van aandacht op lange-termijn habituatie. Met betrekking tot controleerbaarheid wordt benadrukt dat de zekerheid-afleiding hypothese aan een directe toets moet worden onderworpen, te meer omdat andere zekerheid beïnvloedende manipulaties niet de pijn ervaring lijken te beinvloeden. Het falen van psychologische theorieën in het geven van een duidelijke verkla- 
ring voor disproportioneel verhoogde pijn ervaringen, of voor pijn ervaringen zonder enig corresponderend neurologisch pijn signaal, wordt bediscussieerd en enige nieuwe onderzoeksmogelijkheden worden aangeroerd.

Ook m.b.t. de totstandkoming van pijn-anticipatie reacties worden de relatieve bijdragen van de twee bovengenoemde soorten pijn verwachtingen besproken. Ervaringen die beide soorten pijn verwachtingen te boven gaan lijken bij te dragen aan het ontstaan van angst voor pijn, aan de geneigdheid om toekomstige pijn te overschatten, en aan onzekerheid over hoeveel pijn te verwachten. Een directe experimentele vergelijking van de bijdrage van onjuiste pijn verwachtingen van het 'tweede soort' met de bijdrage van snelle (maar voorspelbare) pijn intensiteits verhogingen op zich zou interessant zijn, met name voor de studie naar de totstandkoming van fobische anticipatie reacties (hartslag acceleratie en vermijding). Mogelijke verklaringen voor de asymmetrische effecten van twee typen onjuiste pijn verwachtingen (onder- en overschattingen) worden kritisch besproken. De rol die overschatten en onzekerheid (betrekking hebbend op het gebrek aan voorspelbaarheid van de intensiteit van aversieve gebeurtenissen) spelen in vrees en vermijding wordt behandeld. Er worden suggesties gedaan voor verder onderzoek naar de invloeden van onjuiste pijn verwachtingen op geheugen bias (zijn onderschatte pijnlijke ervaringen beter te herinneren dan correct verwachte ervaringen?) en op de verstoring van latente inhibitie. Ten slotte wordt de mogelijkheid geopperd dat onderzoek naar de specifieke wijzen waarop pijnlijke ervaringen na de eigenlijke ervaring verwerkt worden ons inzicht kan vergroten in de menselijke anticipatie van pijn. 


\section{REFERENCES}

Abramson, L.Y. and Alloy L.B. (1980). Judgements of contingency Errors and their implications. In: A. Baum and J. Singer (Eds.), Advances in Environmental Psychology, Volwme 2. Hillsdale, N.J.: Erlbaum.

Abramson, L.Y., Selligman, M.E.P. and Teasdale, J.D. (1978).) Learned helplessness in humans: critique and reformulation. Journal of Abnormal Psychology, $78,47-49$.

Ahles, T.A., Cassens, H.L. and Stalling, R.B. (1987). Private Body consicousness, anxiety and the perception of pain. Journal of Behavioral Therapy and Experimental Psychiatry, 18, 215-222.

Albersnagel, F.A., Arntz, A. and Gerlsma, C. (1986). Some limitations of the attributional learned helplessness model on understanding effects of (non)-contingency: A controlled study in Dutch adolescents. Advances in Behaviour Research and Therapy, 8, 1-42.

Alloy, L.B. and Abramson, L.Y. (1979). Judgement of contingency in depressed and non-depressed students: sadder but wiser? Journal of Experimental Psychology ${ }_{n} 108$; 441-485.

Alloy, L.B. and Tabachnik, N. (1984). Assessment of covariation by humans and animals: the joint influence of prior expectations and current situational information. Psychological Review, 91, 112-149.

Alloy, L.B., Abramson. L.Y, and Viscusi, D. (1981). Induced mood and the illusion of control. Journal of Personality and Social Psychology, 41, 1129-1140.

Almay, G.L., Johansson, F. and Knorring, won L. (1978). Endorphins in chronic pain; I. differences in CSF endorphin levels between organic and psychogenic pain syndromes. Pain, 5, 153-162.

Armentrout, D.P. (1979). The impact of chronic pain on the self-concept Journal of Clinical Psychology, 35, 517-521.

Arntz, A. and Hout van den, M. (1988). Generalizability of the match/mismatch model of fear. Behaviour Research and Therapy, 26, 207-223.

Arntz, A. and De Jong, P.J. (1988). CLBP and perceived control: an experimental study. In preparation.

Arntz, A. and Schmidt, A.J.M. (1989). Perceived control and the experience of pain. In: A. Steptoe and A. Appels (Eds.), Stress, Personal Control and Health. Chichester: John Wiley, $131-162$.

Arntz, A. and Lousberg, R. (1990). The effects of underestimated pain and their relationship to habituation. Behaviour Research and Therapy, 28, 15-28.

Arntz, A., DeVries, M. and Schmidt, A.J.M. (1988a). Daily experiences of chronic low back pain patients. In preparation.

Arntz, A., Eck, M. van and Heijmans, M. (1990a). Predictions of dental pain: The fear of any expected evil is worse than the evil itself. Behawlour Research and" Therapy, 28, 29-41.

Arntz, A., Eck, M. van, Jong, P. de, and Hout, M.A. van den (1990b). The relationship between underpredicted pain and escape. Behaviour Research and Therapy, 28, 87-90.

Arntz, A., Hout, M.A. van den, Lousberg, R, and Schouten, E. (1990c). Is the match/mismatch model based on a statistical artefact? Behaviour Research and Therapy, 28, 249-253.

Arntz, A., Dreessen, L. and Merckelbach, H. (1991a). Attention, not anxiety, influences pain. Behaviour Research and Therapy, 29, 41-50.

Arntz, A., van Eck, M. \& Jong, P. de (1991b). Avoidance of pain of unpredictable intensity. Behaviour Research and Therapy, 29, 197-201.

Arntz, A., van Eck, M. \& Jong., P. de (1991c). Unpredictable sudden increases in intensity of pain and acquired fear. Journal of Psychophysiology, accepted for publication.

Arntz, A., Merckelbach, H., Peters, M. and Schmidt, A.J.M. (1991d). Chronic low back pain, response specificity and habituation to painful stimuli. Journal of Psychophysiology, 5, in press. 
Aronoff, G.M. and Evans, W.O. (1982). The prediction of treatment outcome at a multidisciplinary pain center. Pain, 14, 67-73.

Atkinson, R.C. and Shiffrin, R.M. (1968). Human memory: a proposed system and its control processes. In: K.W. Spence (Ed.) The Psychology of Learning and Motivation, Volume 2. New York: Academic Press.

Averill, J.R. (1973). Personal control over aversive stimuli and its relationship to stress. Psychological Bulletin, $80,286-303$.

Averill, J.R. and Rosenn, M. (1972). Vigilant and nonvigilant coping strategiles and psychophysiological stress reactions during the anticipation of an electric shock. Journal of Personality and Soctol Psychology, 23, 128-141.

Averill, J.R., O'Brien, L. and DeWitt, G.W. (1977). The influence of response effectiviness on the preference for warning and on psychophysiological stress reactions. Journal of Personality, 45, 396-418.

Avia and Kanfer (1980). Coping with Aversive Stimulation: The effects of training in a selfmanagement context. Cognitive Theropy and Research, 4, 73-81.

Badia, P., Suter, S. and Lewis, P. (1967). Preference for warned shock: Information and/or preparation. Psychological Reports, 20, 271-274.

Badia, P., McBane, B., Suter, S. and Lewis, P. (1966). Preference behavior in an immediate versus variably delayed shock situation with and without a warning signal. Journal of Experimental Psychology, 72, 847-852.

Ball, S. and Vogler, R.E. (1971). Uncertain pain and the pain of uncertainty. Perceptual and Motor Skills, 33, 1195-1203.

Bandura, A. (1986). Self-efficacy mechanism in physiological activation and health-promoting behavior. In: J. Madden IV, S. Matthysse and J. Barchas (Eds.), Adaptation, learning and affect. New York: Raven Press.

Bandura, A., O'Leary, A., Taylor, C.B., Gauthier, J., Gossard, D. (1987). Perceived selfefficacy and pain control: Opioid and Nonopioid Mechanisms. Journal of Personality and Social Psychology, 53, 563-571.

Barlow, D.H. (1985). In: A.H. Tuma and J.D. Maser (Eds.), Anxiety and the Anxiety Disorders. Hillsdale, N.J.: Erlbaum.

Barlow, D.H., Vermilyea, J., Blanchard, E.B., Vermilyea, B.B., DiNardo, P.A. and Cerny, J.A. (1985). The phenomenon of panic. Journal of Abnormal Psychology, 94, 320-328.

Beck, A. (1976). Cognitive therapy and the emotional disorders. New York: International Universities Press.

Beck, A.T., Emery, G. and Greenberg, R.L. (1985). Anxiety disorders and phobias. New York: Basic Books.

Beecher, H.K. (1956). Relationship of significance of wound to pain experienced. Journal of the American Medical Associarion, 161, 1609-1613.

Beers, T.M. and Karoly, P. (1979). Cognitive Strategies, Expectancy, and Coping Style in the Controll of Pain. Journal of Consulting and Clinical Pychology, 47, 1, 179-180.

Bellissimo, A and Tunks, E. (1984). Chronic pain, the psychotherapeutic spectrum. New York: Praeger Publishers.

Berg, 0 . van den (1989). Is it possible to habituate in imagery to fear stimuli? Paper presented at the 19th Annual Congress of the European Association of Behaviour Therapy, Vienna, sept. 2024, 1989 .

Berlyne, D.E. (1960). Conflict, Arousal and Curiosity. New York: McGraw-Hill.

Biederman, G.B., Furedy, J.J. and Heighington, G.A. (1984). The double alternative double start experimental chamber: A new procedure for measuring preference. Journal of Experimental Analysis of Behavior, 42, 137-141.

Biederman, H.J., McGhie, A., Monga, T.N. and Sharks, G.L. (1987). Perceived and actual control in EMG treatment of back pain. Behaviour Research and Therapy, 25, 137-147. 
Bing, F.D. (1961). A practical training course for the psychoprophylactic method of chidbirth. New York: American Society for Psychoprophylaxis in Obstetrics.

Bixenstein, V.E. and Barber, E. (1964). Further analysis of the determinants of avoidance behaviour. Journal of Comparative and Physiology, $58,339-343$.

Björkstrand, P. (1973). Electrodermal responses as affected by subject - versus experimenter controlled noxious stimulation. Journal of Experimental PSychology, 97, 365-369.

Blitz, B., and Dinnerstein, A.J. (1971). Role of attentional focus in pain perception: Manipulation of response to noxious stimulation by instructions. Journal of Abnormal Psychology, $77,42-45$.

Bloom, L.J., Houston, B.K., Holmes, D.J., and Burish, T.S. (1977). The effectiveness of attention diversion and situational redefinition for reducing stress to a nonambiguous threat. Journal of Research in Personality, 11, 83-96.

Blumer, O. and Heilbronn, M. (1976). The pain-prone disorder: a clinical and psychological profile. Psychosomatics, 17, 395-402.

Blumer, O. and Heilbronn, M. (1982). Chronic pain as a variant of depressive disease. Journal of Nervaus and Mental Disease, $170,381-406$.

Bobey, M.J. and Davidson, P.O. (1970). Psychological factors affecting pain tolerance. Journal of Psychosomatic Research, 14, 371-376.

Bohlin; G. and Kjellberg, A. (1979). Orienting activity in two-stimullus paradigms as reflected in heart rate. In: H.D. Kimmel, E.H. van Olst and J.F. Orlebeke (Eds.), The Orienting Reflex in Humans. New York: Wiley, 169-197.

Bolles, R.C. (1978). The role of stimulus learning in defensive behavior. In: S.H. Hulse, H. Fowler \& W.K. Honig (Eds.), Cognitive Processes in Animal Behavior. Lawrence Erlbaum Ass. Hillsdale N.J., 89-107.

Bolles, R.C. and Fanselow, M.S. (1980). A perceptual-defensive-recuperative model of fear and pain. Behavioral and Brain Sciences, 3, 291-323.

Bolles, R.C. and Fanselow, M.S. (1982). Endorphins and behavior, Annual Review of Psychology" $33,87-101$.

Borkovec, T.D. (1985). Worry: A potentially valuable concept. Behaviour Research and Therapy, $23,481-482$.

Bowers, K.S. (1968). Pain, anxiety, and perceived control. Journal of Consulting and Clinical Psychology, 32, 596-602.

Bowers, K.S. (1971). The effect of UCS temporal uncertainty on heart rate and pain. Psychophysiology, 8, 382-389.

Breitner, L. (1971). Anxiety and intolerance of ambiguity. Unpublished study, cited in Epstein (1973).

Brewin, C.R. (1989). Cognitive Foundations of Clinical Psychology. Hove and London: Lawirence Erlbaum.

Broadbent, D.E. (1958). Perception and Comumunication. London: Pergamon Press.

Burger, J.M. and Arkin, R.M. (1980). Prediction, control and learned helplessness. Journal of Personality and Social Psychology, 38, 482-491.

Bush, C., Ditto, B. and Feuerstein, M. (1985). A controlled evaluation of paraspinal biofeedback in the treatment of chronic low back pain. Health Psychology, 4, 307-321.

Buss, A.H. and Portnoy, N.W. (1967). Pain tolerance and group identification. Journal of Personality and Social Psychology, 6, 106-108.

Butler, G. and Matthews, A. (1983). Cognitive processes in anxiety. Advances in Behaviour Research and Therapy, 5, 51-62.

Carlsson, A.H. (1983). Assessment of chronic pain. 1. Aspects of the reliability and validity of the visual analogue scale. Pain, 16,87-101.

Carlsson, A.H. (1984). Assessment of chronic pain. II Problems in the selection of relevant questionnaire items for classification of pain and evaluation and prediction of therapeutic effects. Pain, 19, 173-184. 
Champion, R.A: (1950). Studies of experimentally induced disturbance. Australian Journal of Psychology, 32, 596-602.

Chapman, C.R. (1976). Measurement of pain: Problems and issues. In: J.J. Bonica and D. AlbeFessard (Eds.), Advances in pain research and therapy, Volwne 1. New York: Raven Press.

Chapman, C.R. (1980). Pain and perception: Comparison of sensory decision theory and evoked potential methods. In: J J. Bonica (Ed.), Pain. New York: Raven Press, 111-142.

Chapman, C.R. and Gagliardi, G.J. (1980). Clinical implications of Bolles and Fanselow"s pain/fear model Behavioral and Brain Sciences, 3, 305-306.

Chapman, C.R. and Brena, S.F. (1982). Learmed helplessness and responses to nerve blocks in chronic low back pain patients. Pain, 14, 355-364.

Chapman, C.R. and Bonica, J.J. (1983). Acute Pain. Current Concepts.

Chapman, C.R. and Bonica, JJ. (1985). Chronic pain (Current Concepts). Kalamazoo, MI: The Upjohn Co.

Chapman, C.R. and Turner, J.A. (1986). Psychological Control of Acute Pain. Journal of Pain and Symptom Management, 1, 9-20.

Chaves, J.F. and Brown, J.M. (1987). Spontaneous cognitive strategies for the control of clinical pain and stress. Journal of Behavioral Medicine, 10, 263-276.

Chertok, L. (1959). Psychosomatic Methods in painless childbirth. New York: Pergamon Press.

Chew, A.C.N., Chapman, C.R. and Harkins, S.W. (1979). Brain evoked potentials are functional correlates of induced pain in man. Pain, 6, 365-374.

Clark, D.M. (1983). On the induction of depressed mood in the laboratory: evaluation and comparison of the Velten and Musical Procedures. Advances in Behaviour Research and Therapy, 5, 27-49.

Clark, D.M. and Teasdale J.D. (1985). Constraints on the effects of mood on memory. Journal of Personality and Social Psychology, 48, 1595-1608.

Cogan, R., Hennebron, W. and Klopfer, F. (1976). Predictors of pain during prepared childbirth. Journal of Psychosomatic Research, 20, 523-533.

Coles, M.G.H., Herzberger, S.D., Sperber, B.M. and Goetz, T.E. (1975). Physiological and behavioral concomittants of mild stress: The effects of accuracy of temporal information. Journal of Research in Personality, 9, 168-176.

Collins, G.A., Cohen, M.J., Naliboff, B.D. and Schandler, S.L. (1982). Comparative analysis of paraspinal and frontalis EMG, heart rate and skin conductance in chronic low back pain patients and normals to various postures and stress. Scandinavian Journal of Rehabilitation Medicine, 14, 39-46.

Cook, J.O. and Barnes, L.W., Jr. (1964). Choice of delay of inevitable shock. Journal of Abnormal and Social Psychology, 68, 669-672.

Corah, N.L. (1973). Effects of Perceived Control on stress reductions in pedodontic patients. Journal of Dental Research, 52, 1261-1264.

Corah, N.L. and Boffa, J. (1970). Perceived control, self-observation, and response to aversive stimulation. Journal of Personality and Social Psychology, 16, 1-4.

Corah, N.LL.., Bissell, D. and Illig, S.J. (1978). Effect of perceived control on stress reduction in adult dental patients. Journal of Dental Research, 57, 74-76.

Corah, N.L., Gale, E.N. and Illig, S.J. (1979). Psychological stress reduction during dental procedures. Journal of Dental Research, 58, 1347-1351.

Corey, D. (1989). Pain. learning to live without it. New York: Plume.

Cornwall, A, and Donderi, D.C. (1988). The effect of experimentally induced anxiety on the experience of pressure pain. Pain, 35, 105-113.

Craske, M.G., Rapee, R.M. and Barlow, D.H. (1988). The significance of panic-expectancy for individual patterns of avoidance. Behawior Therapy, 19, 577-592.

D"Amato, M.R. and Gumenik, W.E. (1960). Some effects of immediate versus randomly delayed shock on an instrumental response and cognitive processes. Journal of Abnormal and Social Psychology, 60, 64-67. 
D'Amato, M.R. and Safarjan, W.R. (1979). Preference for information about shock duration. Animal Learning and Behaviour, 7, 89-94.

Dantzer, R. (1989). Neuroendocrine correlates of control and coping. In: A. Steptoe and A. Appels (Eds.), Stress, Personal Control and Health, 277-294. Chichester: Wiley.

Davey, G.C.L. (1989). UCS revaluation and conditioning models of acquired fears. Behaviour Research and Therapy, 27, 521-528.

Davey, G.C.L. (1989). Dental phobias and anxieties: evidence for conditioning processes in the acquisition and modulation of dental fear. Behaviour Research and Therapy, 27, 51-58.

Davies, P. (1987). Conditioning and perception. In: G. Davey (Ed.), Cognitive Processes and Pavlovian Conditioning in Humans. Chichester: J. Wiley, 183-210.

Davison, G.C. and Valins, S. (1969). Maintenance of self-attributed and drug-attributed behavior change. Journal of Personality and Social Psychology, 11, 25-33.

DeGood, D.E. (1975). Cognitive Control Factors in Vascular stress response. Psychophysiology, $12,399-401$.

De Wilde, G.J.S. (1983). Neurotische labiliteit gemeten volgens de vragenlijst methode. (Neurotic Lability Measured by Means of Questionnaire.) Amsterdam: Van Rossen.

Di Cara, L.V., Braun, J.J., and Pappas, B.A. (1970). Classical conditioning and instrumental learning of cardiac and gastrointestinal responses following removal of neocortex in the rat. Journal of Comparative and Physiological Psychology, 73, 208-216.

Dimitrijevic, M.R., Faganel, J., Gregoric, M., Nathain, P.W. and Tronelj, J.K. (1972). Habituation effects of regular and stochastic stimulation. Journal of Newrology, Neurosurgery and Psychiatry, 35, 234-242.

Dolce, J.J. (1987). Self-efficacy and disability beliefs in behavioral treatment of pain. Behaviour Research and Pain, 25, 4, 289-299.

Donegan, N.H. and Wagner, A.R. (1987). Conditioned diminution and facilitation of the UR: A

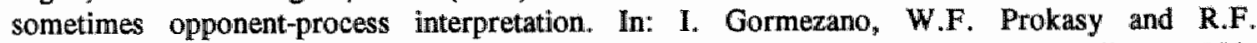
Thompson (Eds.), Classical Conditioning (Rev. Ed.). Hillsdale, N.J.: Lawrence Erlbaum, 339369.

Dougher, M.J. (1979). Sensory decision theory analysis of the effects of anxiety and experimental instructions on pain. Journal of Abnormal Psychology, 88, 137-144.

Dougher, M.J., Goldstein, D., and Leight, K.A. (1987). Induced anxiety and pain. Journal of Anxiety Disorders, $1,259-264$.

Eikelboom, R. and Stewart, J. (1982). Conditioning of drug-induced physiological responses. Psychological Review, 89, 507-528.

Elliot, R. (1969). Tonic heart rate: Experiments on the effects of collative variables lead to a hypothesis about its motivational significance. Journal of Personality and Social Psychology. $12,211-228$.

Elton, D., Stanley, G.V. and Burrows, G.D. (1978). Self-esteem and chronic pain. Journal of Psychosomatic Research, 22, 25-30.

Engel, G.L. (1959). "Psychogenic" pain and the paimprone patient. American Journal of Medicine, 26, 899-918.

Epstein, S. (1971). Heart rate, skin conductance, and intesnity ratings during experimentally induced anxiety: Habituation within ong days. Psychophysiology, 8, 187-197.

Epstein, S. (1973). Expectancy and magnitude of reaction to a noxious UCS. Psychophysiology, 10, 100-107.

Epstein, S. and Clarke, S. (1970). Heart rate and skin conductance during experimentally induced anxiety: effects of anticipated intensity of noxious stimulation and experience. Journal of Experimental Psychology, 84, 105-112.

Epstein, S. and Roupenian, A. (1970). Heart rate and skin conductance during experimentally induced anxiety: the effect of uncertainty about receiving a noxious stimulus. Journal of Personality and Social Psychology , 16, 20-28. 
Epstein, s. and Kling, J.S. (1971). Reactivity during awictpation and impact of an unavoidable noxious stimulus as a function of time uncertatity. Unpublished study, cited in Epstein (1973).

Eysenck, HJ. (1967). The Blological Basis of Persionality. Springfield: Thomas.

Eysenck, H.I. (1979). The conditioning model of neurosis, Behavioral and Brain Sciences, 2, 155199.

Eysenck; H.J. (1980). Fear, pain and anxiety. Behovioral and Brain Sciences, 3, 307-308.

Eysenck, H. . (1986), A theory of the incubation of anxiety/fear responses. Behaviour Research and Therapy, $6,309-321$.

Fanselow, M.S. (1980). Signaled shock-free periods and preference for signaled shocks. Journal of Experimental Psychology: Animal Behavior Processes, 6, 65-80.

Fanselow, M.S. and Bolles, R.L. (1979). Triggering of the endorphin analgesia reaction by a cue previcuisly associated with shock: Reversal by Naloxone. Bulletin of the Psychonomic Society, 14, 88-90.

Feinman, $\mathrm{Ch}$. (1985). Pain relief by antidepressants: possible modes of action. Pain, 23, 1-8.

Felton, G. and Segelman, F. (1978). Lamaze childbirth training and changes in belief about personal control. Birth and the Founily Journal, 5, 141-150.

Feuerstein, M., Papciak, A.S. and Hoon, P.E. (1987). Biobehavioral mechanisms of chronic low back pain. Clinical Psychology Review, 7, 243-273.

Finkelman, J.M. and Glass, D.C. (1970). Reappraisal of the relationship between noise and human performance by means of a subsidiary task measure. Journal of Applied Psychology, 54, 211213.

Fisher, S. (1986). Stress and strategy. London: Lawrence Erlbaum.

Flor, H., Haag, G., Turk, D.C. and Koehler, H. (1983). Efficacy of EMG biofeedback pseudotherapy, and conventional medical treatment for chronic rheumatic back pain. Pain, 17, 21-31.

Fordyce, W.E. (1974). Pain viewed as learned behaviour.. In: J.J. Bonica (Ed.), Advances in Neurology, Volume 4, 415-422. New York: Raven Press.

Fordyce, W.E. (1976). Behavioral Methods in chronic pain and illness. St. Louis: Mosby.

Fordyce, W.E., Roberts, A.H. and Sternbach, R.A. (1985). The behavioral management of chronic pain: a response to critics. Pain, 22, 113-125.

Fordyce, W., Lansky, D., Calsym, D., Shelton, J., Stolow, W. and Rock, D. (1984). Pain measurement and pain behavior. Pain, 18, 53-69.

Fowles, D.C., Christie, M.J., Edelberg, R., Grings, W.W., Lykken, D.T. and Venables, P.H. (1981). Committee report. Publication recommendations for electrodermal measurements. Psychophysiology, 18, 232-239.

Fredrikson, M. (1983). Reliability and validity of some specific fear questionnaires. Scandinavian Journal of Psychology, 24, 331-334.

Freeman, J. and Badia, P. (1975). Do rats prefer information about shock intensity? Bulletin of the Psychonomic Sociery; $6,75-78$.

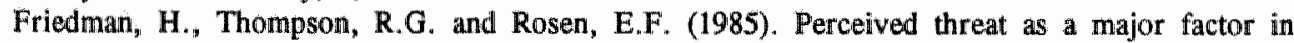
tolerance for experimentally induced cold-water pain. Journal of Abnormal Psychology, 94, 624-629.

Furedy, J.J. (1970). Test of the preparatory adaptive response interpretation of aversive classical autonomic conditioning. Journal of Experimental Psychology, 84, 301-307.

Furedy, J.J. (1973). Auditory and autonomic tests of the preparatory-adaptive-response interpretation of classical aversive conditioning. Journal of Experimental Psychology, 99, 280-283.

Furedy, J.J. (1975). An integrative progress report on informational control in humans: some laboratory findings and methodological claims. Australian Journal of Psychology, 27, 61-83.

Furedy, J.J. and Chan, R.M. (1971). Failures of information to reduce rated aversiveness of unmodifiable shock. Australlian Journal of Psychology, 23, 85-94.

Furedy, J.J. and Doob, A.N. (1971a). Autonomic responses and verbal reports in further tests of the preparatory-adaptive-response interpretation of reinforcement. Journal of Experimental Psychology, 89, 258-264. 
Furedy, J.J. and Doob (1971b). Classical aversive conditioning of human digital volume-pulse change and tests of the preparatory-adaptive-response interpretation of reinforcement. Jowmal of Experimental Psychology. 89, 403407.

Furedy, J.J. and Doob, A.N. (1972). Signalling unmodifiable shocks: Limits on human informational cognitive control. Journal of Personality and Social Psychology, $21,111-115$.

Furedy, J.J. and Ginsberg, S. (1973). Effects of varying signalling and intensity of shock on an unconfounded and novel electrodermal autonomic index in a variable and long-interval classical trace conditioning paradigm. Psychophysiology, 10, 328-334.

Furedy, J.J. and Klajner, F. (1972a). Preference, verbal ratings and autonomic data on the preparatory-response thsory: Subjects believe in it, but it is false. Paper presented at the 7th Annual Conference of the Australian Psychological Society. Canberra, August 1972. (cited in Furedy, 1975).

Furedy, J.J. and Klajner, F. (1972b). Unconfounded autonomic indices of aversiveness of signalled and unsignalled shock. Joumal of Experimental Psychology, 93, 313-318.

Furedy, J.J., Riley, D.M. and Fredrikson, M. (1983). Pavlovian extinction, phobias, and the limits of the cognitive paradigm. The Pavlovian Journal of Biological Science, 18, 126-135.

Furedy, J.J., Fainstat, D., Kulin, P., Lasko, L. and Nichols, S. (1972). Preparatory-response vs. information-seeking interpretations of preference for signalled loud noise: Further limits on human informational cognitive control. Psychonomic Science, 27, 108-110.

Gaebelin, J., Taylor, S.P. and Borden, R. (1974). Effects of an external cue on psychophysiological reactions to an external event. Psychophysiology, 11, 315-320.

Gatchel, R.J. and Proctor, J.D. (1976). Physiobiological correlates of עearned helplessness in man. Journal of Abnormal Psychology, 85, 27-34.

Gatchel, R.J., McKinney, M.E. and Koebernick, L.F. (1977). Learned Helplessness, Depression and Physiologic Responding. Psychophysiology, 14, 25-31.

Geer, J.H. and Maisel, E. (1972). Evaluating the effects of the prediction-control confound. Journal of Personality and Sacial Psychology, 23, 314-319.

Geer, J.H., Davison, G.C. and Gatchel, R.J. (1970). Reduction of stress in humans through nonveridicall perceived control of aversive stimulation. Journal of Personality and Social Psychology, 16, 731-738.

Girodo, M. and Wood, D. (1979). Talking yourself out of pain: The importance of believing that you can. Cognitive Therapy and Research, 3, 1, 23-33.

Glass, D.C., Singer, J.E. and Friedman, L.N. (1969). Psychic cost of adaptation to an environmental stressor. Journal of Personality and Social Psychology, 12, 200-210.

Glass, D.C., Reim, B. and Singer, J.E. (1971). Behavioral consequences of adaptation to controllable and uncontrollable noise. Journal of Experimental and Social Psychology, 7, 244. 257.

Glass, D.C., Singer, J.E., Leonard, H.S., Krantz, D., Cohen, S. and Cummings, H. (1973). Perceived control of aversive stimulation and the reduction of stress responses. Journal of Personality, 41, 577-595.

Gouaux, C. and Gouaux, S.M. (1971). The influence of induced affective states on the effectiveness of social and non-social reinforcers in an instrumental learning task. Psychonomic Science, $22,341-343$.

Gracely, R.H. (1979). Psychophysical assessment of human pain. In: J.J. Bonica, J.C. Liebeskind, D.G. and Albe-Fessard, (Eds.), Advances in pain research and therapy, 805-824.

Gracely, R.H., Dubner, R., Wolskee, P.J. and Deeter, W.R. (1983). Placebo and naloxone can alter post-surgical pain by separate mechanisms. Nature, 306, 264-265.

Graham, F.K. (1978). Constraints an measuring heart rate and period through real and cardiac time. Psychophysiology, 15, 492-495.

Gransier, S. \& Arntz, A. (1991). Chronic low back pain and learned helplessness. An experimental test. In preparation.

Gray, J.A. (1975). Elements of a Two-Process Theory of Learning. London: Academic Press. 
Gray, J.A. (1976). The behavioral inhibition system: a possible substratum for anxiety. In: M.P. Feldman, and A. Broduhurst, (Eds.). Theoretical and Experinental Bases of the Behaviour Therapies. London: Wiley, $3-41$.

Gray, J.A. (1982). The newropsychology of anxiety. Oxford: Oxford University Press.

Gray, J.A. (1985). Issues in the newropsychology of anxiety. In: A. Tuma, and I. Maser, (Eds.). Anxiety and the anxiety disorders. Hillsdalle, N J.: Erlbaum.

Grimm, L. and Kanfer, F.H. (1976). Tolerance of Aversive Stimulation. Behavior Therapy, 7, $593-601$.

Grings, W.W. and Schell, A.M. (1971). Effects of trace versus delay conditioning, interstimulus interval variability, and instructions on UCR diminution. Journal of Experimental Psychology, 90, 136-140.

Groves, P.M. and Thompson, R.F. (1970). Habituation: A dual-process theory. Psychological Review; 77, 419-450.

Haggard, E.A. (1949). Experimental studies in affective processes: I. Some effects of cognitive structure and active participation on certain autonomic reactions during and following experimentally induced stress. Journal of Experimental Psychology, 33, 257-284.

Haley, W.E., Turner, J.A. and Romano, J.M. (1985), Depression in chronic pain patients: relation to pain, activity, and sex differences. Pain, 23, 377-343.

Hanvik, L.I. (1951). M.M.P.I profiles in patients with low back pain. Journal of Consulting Psychology, 15, 350-353.

Hare, R.D. and Blewings, G. (1975). Conditioned orienting and defensive respones. Psychophysio$\log y, 77,223-239$.

Haslam, D.R. (1966). The effect of threatened shock upon pain threshold. Psychonomic Sclence, 6, 309-310,

Headrick, M.W. and Graham, F.K. (1969). Multiple-component heart rate responses conditioned under paced respiration. Journal of Experimental Psychology, 79, 486-494.

Higgins S.T. and Morris $\mathbb{E} . K$. (1984). Generality of free-operant avoidance conditioning to human behavior. Psychological Bulletin, 96, 247-272.

Hilgard, E.R. (1975). The alleviation of pain by hypnosis. Pain, 1, 213-231.

Hokansion, J.E., DeGood, D.E., Forrest, M.S. and Brittain, T.M. (1971). Availabillity of avoidance behaviors in modulating vascular-stress responses. Journal of Personality and Social Psychology, 19, 60-86.

Holroyd, K.A., Penzien, D.B., Hursey, K.G., Tobin, D.L., Rogers, L., Holm, J.E., Mancille, P.J., Hall, J.R. and Chila, A.G. (1984). Change mechanisms in EMG biofeedback training: Cognitive changes underlying improvements in tension headache. Journal of Consulting and Cinical Psychology, 52, 1039-1053.

Holzman, A.D., Turk, D.C. and Kerns, R.D. (1986). The cognitive-behavioral approach to the management of chronic pain. In: A.D. Holzman and D.C. Turk (Eds.), Pain Management. New York: Pergamon, 31-50.

Houston, B.K. (1972). Control over stress, locus of control, and response to stress. Journal of Personality and Social Psychology, 21, 249-255.

Hout, M.A. van den, Arntz, A., Hoekstra, R., and Schouten, E. (1989). In preparation.

Iacono, W.G. and Lykken, D.T. (1984). The effects of instructions and an engaging visual task on habituation to loud tones: An evaluation of an alternative to the traditional habituation paradigm. Physiological Psychology, 12, 23-29.

Imada, H. and Nageishi, $Y_{*}(1982)$. The concept of uncertainty in animal experiments using aversive stimulation. Psychological Bulletin, 91, 573-588.

International Association for the Study of Pain (IASP) (1979). Pain terms: a list with definitions and notes on usage. Pain, 6, 249-252.

International Association for the Study of Pain, subcommittee on Taxonomy. Classification of chronic pain; describtions of chronic pain syndromes and definitions of pain terms. (1986). Pain, 3, S1-S226 (suppl.11). 
Jackson, R.L, Maier, S.F, and Coon, D.J. (1979). Long-term analgesic effects of inescapable shocks and learned helplessness. Science, 209, 91-94.

Jacobsson, $\mathbb{E}$. (1959). How to relax and have your baby. New York: MoGraw-Hill.

Jensen, J. (1988). Life events in neurological patients with headache and low back pain (in rellation to diagnosis and persistence of pain). Pain, $32,47-53$.

Jensen, M.P., Karoly, P. and Braver, S. (1986). The measurement of clinical pain intensity: a comparison of six methods. Pain, 27, 117-126.

Johnson, J.E. (1973). Effects of accurate expectations about sensations on the sensory and distress components of pain. Journal of Personality and Social Psychology, 27, 261-275.

Johnson, J.E., Morrisey, J.F. and Leventhal, H. (1973). Psychological preparation for an endoscopic examination. Gastrointestinal Endoscopy, 19, 180-182.

Jones, K.E. and Furedy, J.J. (1989). Effects of signalling parameters on human electroshock perception: informational control versus stimulus error. Australian Journal of Psychology, 4I, 79-87.

Jones, T. \& Dawey, G.C.L. (1990). The effects of eued UCS rehearsal on the retention of differential "fear" conditioning: An experimental analogue of the "worry" process. Behaviour Research and Therapy, 28, 159-164.

Jones, A., Bentler, P.M. and Petry, G. (1966). The reduction of uncertainty concerning future pain. Journal of Abnormal Psychology, 71, 87-94.

Jong, P.J. de, Merckelbach, H. and Arntz, A. (1991). Illusory correlation, on-line probability estimates, and ellectrodermal responding in a (quasi) conditioning paradigm. Biological Psychlogy, in press.

Kahneman, D. (1973). Attention and Effort. Englewood Cliffs, N.J.: Prentice-Hall.

Kanfer, F.H. and Goldfoot, D.A. (1966). Self-control and tolerance of noxious stimulation. Psychological Reports, 18, 79-85.

Kanfer, F.H. and Seidner, M.L. (1973). Self-control: factors enhancing tolerance of noxious stimulation. Journal of Personality and Social Psychology, 25, 381-389.

Karmel, M. (1965). Thank you Dr. Lamaze. New York: Delphin Books,

Katz, $\mathbb{R}$. (1984). Unconfounded electrodermal measures in assessing the aversiveness of predictable and unpredictable shocks. Psychophystology, 21, 452-458.

Kent, G. (1984). Anxiety, pain and type of dental procedure. Behaviour Research and Therapy, $22,465-469$.

Kent, G. (1985). Memory of dental pain. Pain, 21, 187-194.

Kent, G. (1986). The typicality of therapeutic "surprises" Behaviour Research and Therapy, 24, 625-628.

Kent, G. (1987). Self-efficacious control over reported physiological, cognitive and behavioural symptoms of dental anxiety. Behaviour Research and Therapy, 25, 341-347.

Kent, G. and Warren, P. (1985). A study of factors associated with changes in dental anxiety. Journal of Dental Research, 64, 1316-1318.

Kilminster, S.G. and Jones, D.M. (1986). Perceived control and the cold pressor test. Stress Medicine, 2, 73-77.

Kimmel, H.D. (1966). Inhibition of the unconditioned response in classical conditioning. Psychological Review, 73, 232-240.

Kimmel, E. (1967). Judgements of UCS intensity and diminution of the UCR in classical GSR conditioning. Journal of Experimental Psychology, 73, 532-543.

Kimmel, H.D. and Pennypacker, H.S. (1962). Conditioned diminution of the uncondioned response as a function of the number of reinforcements. Journal of Experimental Psychology. 64, 20-23.

Kleinknecht, R.A. and Bernstein, D.A. (1978). The assessment of dental fear. Behavior Therapy, 9, 626-634.

Klemp, G.O. and Rodin, J. (1976). Effects of uncertainty, delay, and focus of attention on reactions to an aversive situation. Journal of Experimental Social Psychology, 12, 416-421. 
Klepac, R.K. Dowling, I. and Hange, G. (1982). Characteristics of clients seeking therapy for the reduction of dental avoidance: reactions to pain. Journal of Behavior Therapy and Experimental Psychlatry, 13,293-300.

Klepac, R.K. McDonald, M. Hange, G. and Dowling, J. (1980). Reactions to pain among subjects high and low in dental fear. Journail of Behavioral Medicine, 3, 373-384.

Klorman, R., Weerts, Th.C., Hastings, J.E., Melamed, B.G., Lang, P. (1974). Psychometric description of some specific fear questionnaires. Behowior Therapy, 5, 401-409.

Lang, P. (1985). The cognitive psychophysiology of emotion: Fear and anxiety. In: A.H. Tuma and I.D. Maser (Eds.), Anxiety and the Anxiety Disorders. Hillsdale, N.J.: Lawrence Erlbaum, $247-272$.

Lanzetta, J.T. and Driscoll, J.M (1966). Preference for information about an uncertain but unavoidable outcome. Journal of Personality and Social Psychology, 3, 96-102.

Latimer, P.R. (1982). External contingency management for chronic pain: critical review of the evidence. American Journall of Psychiatry, 139, 1308-1312.

Lautsch, H. (1971). Dental phobia. British Journal of Psychiary, 119, 151-158.

Lavy, E., Hout, M.A. van den and Arntz, A. (1990). Prediction of aversive events. Effects of matches and mismatches in agoraphobic and neutral situations. Behaviour Research and Therapy, 28, 43-50.

Lepanto, R., Moroney, W. and Zenhausern, R. (1965). The contribution of anxiety to the laboratory investigation of pain. Psychonomic Science, 3, 475-476.

Lethem, J., Slade, P.D., Troup, J.D.G. and Bentley, G. (1983). Outline of a fear-avoidance model of exaggerated pain perception. Behaviour Research and Therapy, 21, 401-408.

Leventhal, H., Nerenz, D.R. and Strauss, A. (1980). Self-regulation and the mechanisms for symptom appraisal. In: D. Mechanic (Ed.), Psychosocial Epidemiology. New York: Neal Watson Academic Publications, 55-86.

Leventhal, H., Brown, D., Shacham, S. and Engquist, G. (1979). Effects of preparatory information about sensations, threat of pain, attention on cold pressor distress. Journal of Personality and Social Psychology, 37, 688-714.

Levey, (1980). Measurements units in psychophysiology. In: I. Martin and P.H. Venables (Eds.), Techniques in Psychophysiology. New York: Wiley, 597-628.

Levine, J.D., Gordon, N.C. and Fields, H.L. (1978). The mechanism of placebo analgesia. Lancet, 2, 654-657.

Liddell, A. and May, B. (1984). Some characteristics of regular and irregular attenders for dental check-ups. British Journal of Clinical Psychology, 23, 19-26.

Lindsay, S.J.E. (1983). The fear of dental treatment: a critical and theoretical analysis. In: S. Rachman (Ed.), Contributions to Medical Psychology, 3, Oxford: Pergamon Press.

Lindsay, $\mathbf{P}_{4} \mathbf{H}$. and Norman, D.A. (1977). Human Information Processing. An Introduction to Psychology. New York: Academic Press.

Linton, S.J. (1986). Behavioral remediation of chronic pain: a status report. Pain, 24, 125-141.

Litt , M.D. (1988). Self-efficacy and perceived control: cognitive mediators of pain tolerance. Journal of Personality and Social Psychology, 54, 149-160.

Lovibond, S.H. (1968). The aversiveness of uncertainty: An analysis in terms of activation and information theory. Australian Journal of Psychology, 20, 85-91.

Lykken, D.T. (1959). Preliminary observation on the preception phenomenon. Psychophysiological Measurements Newsletter, 5, 2-4.

Lykken, D.T. (1967). Valins" "emotionality and autonomic reactivity": an appraisal. Journal of Experimental Research in Personality, 2, 49-55.

Lykken, D.T. (1968). Neuropsychology and psychophysiology in personality research. In: E.F. Borgatta \& W.W. Lambert (Eds.), Handbook of personality theory and research. New York: Rand McNally, 413-509.

Lykken, D.T. (1972). Range correction applied to heart rate and GSR data. Psychophysiology. 9, 373-379. 
Lykken, D.T. and Tellegen (1974), On the validity of the preception hypothesis. Psychophysiolo* $g y, 11,125-132$.

Lykken, D.T., Macindoe, J and Tellegen, A. (1972). Preception: autonomic response to shocks as a function of predictability in time and locus. Psychophysiology, 9, 318-333.

Lynn, $_{3}$ B. and Perl, E.R. (1977). A comparison of four tests for assessing the pain sensitivity of different subjects and test areas. Pain, 3, 353-365.

Mackintosh, N.J. (1987). Neurobiology, psychology and habituation. Behaviour Research and Therapy, 25, 81-97.

MacLennan, A.J., Jackson, R.L., and Maier, S.F. (1980). Conditioned analgesia in the rat. Bulletin of the Psychonomic Society, 15, 387-390.

Magora, A. (1973). Investigation of the relation between low back pain and occupation. Scandinavian Journal of Rehabilitation Medicine, 5, 191-196.

Maier, S.F. (1986). Stressor controllability and stress-induced analgesia. Annals New York Academy of Sciences, 467, 55-72.

Maier, F. and Seligman, M.E.P. (1976). Learned helplessness: theory and evidence. Journal of Experimental Psychology: General, 105, 3-46.

Maier, S.F., Sherman, J.E., Lewis, J.W., Terman, G.W. and Liebeskind, J.C. (1983). The opioid/nonopioid nature of stress-induced analgesia and learned helplessness. Journal of Experimental Psychology, Animal Behavior Processes, 9, 80-90.

Malow, R.M. (1981). The effects of induced anxiety on pain perception: A signal detection analysis. Pain, $11,397-405$.

Malow, R.M., West, J.A. and Sutker, P.B. (1987). A sensory decision theory analysis of anxiety and pain responses in chronic drug abusers. Journal of Abnormal Psychology, 96, 184-189.

Malow, R.M., West, J.A. and Sutker P.B. (1989). Anxiety and pain response changes across treatment: sensory decision analysis. Pain, 38, 35-44.

Maltzman, L. and Wolff, C. (1970). Preference for immediate versus delayed noxious stimulation and the concomitant GSR. Journal of Experimental Psychology, 83, 76-79.

Manning, M.M. and Wright, T.L. (1983). Self-efficacy expectancies, outcome expectancies, and the persistence of pain control in child birth. Journal of Personality and Social Psychology, 45, $421-431$.

Marks, I.M. (1987). Fears, phobias and rituals. New York: Oxford University Press.

Marlin, N.A., Sullivan, J.M., Berk, A.M. and Miller, R.R. (1979). Preference for information about intensity of signaled tailshock. Learning and Motivation, 10, 85-97.

Martin, I. \& Levey, A.B. (1987). Learning what will happen next: conditioning, evaluation and cognitive processes. In: G.Davey (Ed.), Cognitive processes and Pavlovian conditioning. Chichester: John Wiley, 57-82.

Martin, P.M., Nathan, P.R., Milech, D. and wan Keppel, M. (1988). The relationship between headaches and mood. Behoviour Research and Therapy, 26, 353-356.

Maruta, T., Swanson, D.W. and Swenson, W.M. (1979). Chronic pain: which patients may a paili management program help? Pain, 7, 321-329.

Matthews, B.A., Shimoff, E., Catania, A.C. and Sagvolden, T. (1977). Uninstructed human responding: sensitivity to ratio and interval contingencies. Journal of the Experimental Amalysis of Behavior, 27, 453-467.

Matthews, K.A., Scheier, M.F., Brunson, B.I. and Carducei, B. (1980). Attention, unpredictability and reports of physical symptoms: eliminating the benefits of predictability. Journal of Personality and Social Psychology, 38, 525-537.

McGreary, Ch. and Turner, J. (1984). Locus of control, regression-sensitization, and psychological disorder in chronic pain patients. Journal of Clinical Psychology, 40, 897-901.

Melzack, R. (1973). The Puzzle of Pain. New York: Basic Books.

Melzack, R. (1975). The McGill pain questionnaire: major properties and scoring methods. Pain, 1, 277-299.

Melzack, $\mathbb{R}_{.} \mathbf{M}_{n}$ (1980). Psychological Aspects of pain. Paln, New York: Raven Press, 143-154. 
Melzack, R. (Ed.) (1983). Pain measurement and assessment. New York: Raven Press.

Melzack, R. and Wall, P.D. (1965). Pain mechanisms: a new theory. Sclence, 150, 971-979.

Merckelbach, H, and Jong, P. de (198\%). Geconditioneerde inhibitie van psychofysiologische reacties op pijnprikkels: feiten en speculaties (Conditioned inhibition of psychophysiological responses to painful stimuli: facts and suggestions). Psychologüca Belgica, 28, 139-155.

Marckelbach, H., Hout, M.A. wan den, and Molen, G.M. van der (1987). Fear of animals: correlations between fear ratings and percelved characteristics. Psychological Reports, 60, 1203-1209.

Merckelbach, H, Ruiter, C. de, Hout, M.A. van den, and Hoekstra, R. (1989). Conditioning experiences and phobias. Behaviour Research and Therapy, 27, 657-662.

Miller, S.M. (1979). Controllability and humam stress: method, evidence and theory. Behaviour Research and Therapy, 17, 287-304.

Miller, S.M. (1981). Predictability and human stress: toward a clarification of evidence and theory. In: L. Berkowitz (Eds.), Adwances in Experimental Social Psychology, 14, 203-256.

Miller, S.M. and Crant, R.P. (1979). The blunting hypothesis: A view of predlictability and human stresss. In: P.O. Sjöden, S. Bates and W.S. Dochens (Eds.), Trends in Behaviour Therapy. New York: Academic Press.

Mills, R.T. and Krantz, D.S. (1979). Information, choice and reactions to stress: A field experiment in a blood bank with laboratory analogue. Journal of Personality and Social Psychology, 4, 608-620.

Mineka, S. (1985) Animal models of anxiety-based disorders: their usefulness and limitations. In: A.H. Tuma and J.D. Maser (Eds.), Anxiety and the Anxiety Disorders. Hillsdalle, N.J.: Erlbaum, 199-244.

Mineka, $\mathbf{S}$. and Kihlstrom, J.F. (1978), Umpredictable and uncontrollable stress: A mew perspective on experimental neurosis. Journal of Abnormal Psychology, 87, 256-271.

Mineka, $\mathbf{S}$. and Hendersen, R.W. (1985). Controllability and predictability in acquired motiwation. Annual Review of Psychology, 36, 495-529.

Mineka, S. and Tomarken, A.J. (1989). The role of cognitive biases in the origins and maintenance of fear and anxiety disorders. In: T. Archer and L.G. Nilson (Eds.), Aversion, avoidance, and anxiety: Perspectives on aversively motivated behavior. Erlbaum, Hillsdale, N.J., 195-221.

Monat, A., Averill, J.R. and Lazarus, $\mathbb{R}$.S. (1972). Anticipatory stress and coping reactions under various conditions of uncertainty. Journal of Personality and Social Psychology, 24, 237-253.

Morrow, M.C. (1966). Recovery of conditioned UCR diminution following extinction. Journal of Experimental Psychology, 71, 884-888.

Mowrer, O.H. (1939). A stimulus-response analysis of anxiety and its role as a reinforcing agent. Psychological Review, 45, 553-564.

Mulcahy, R.A. and Janz, N. (1973). Effectiweness of Raising Pain Perception Threshold in Males and Females Using a Psychoprophylactic Childbirth Technique during Induced Pain. Nursing Research, 22, 5, 423-427.

Nagi, S.Z., Riley, L.E. and Newby, L.G. (1973). A social epidemiology of back pain in a general population. Journal of Chronic Diseose, 27, 769-779.

Nalibof, B.D., Cohen, M.J., Schandller, S.L. and Heinrich, R.L. (1981). Signal detection and thresthold measures for chronic back pain patients, chronic illness patients, and cohort controls to radiant heart stimuli. Journal of Abmormal Psychology, 90, 271-274.

Neufeld, R.W.I. and Thomas, P. (1977). Effects of perceived efficacy of a prophylactic controllling mechanism on self-control under pain stimulation. Canadian Journal of Behavioural Silence, 9, 224-232.

Nisbett, R.E. and Ross, L. (1980). Human Inference: Strategies and Shortcomings of Social Judgements. Englewood Cliffs, N.J.: Prentice Hall.

Nouwen, A. and Solinger, J.W. (1979). The effectiveness of EMG Biofeedback training in low back pain. Biofeedback and Self-Regulation, $4,103=11$. 
O'Brien, R.G. and Kister Kaiser, M. (1985). MANOVA Method for analyzing repeated measures designs: an extensive primer. Psychological Bullerin, 97, 316-333.

Obrist, P.A. (1981). Cardiowascular Psychophysiology, a Perspective. New Yoirk: Plenum Press.

Obrist, P.A., Wood, D.W., and Perez-Reyes, M. (1965). Heart rate during conditioning in humans: effects of UCS intensity, vagal blockade, and andrenergic block of vasomotor activity. Journal of Experimental Psychology, 70, 32-42.

Obrist, P.A., Webb, R.A. and Sutterer, J.R. (1969). Heart rate and somatic changes during aversive conditioning and a simple reaction time task. Psychophysiology $5,696-723$.

Öhman, A. (1979). The orienting response, attention and learning: an information-processing perspective. In: H. Kimmel, E.H. van Olst and J.F. Orlebeke (Eds), The Orienting Reflex in Humans. Hillsdale, N.J.: Erlbaum, 443-471.

Osgood, C.E. (1962). Studies on the generality of affective meaning systems. American Psychologist, 17, 10-28.

Ost, L.G. (1985). Ways of acquiring phobias and outcome of behavioural treatments. Behaviour" Research and Therapy, 23, 683-689.

Öst, L.G. (1989). One session treatment of specific phobias. Behaviour Research and Therapy, 27, $1-7$.

Overmier, J.B., Patterson, Jo and Wielhiewicz, R.M. (1980). Environmental contingencies as sources of stress in animals. In: S. Levine and H. Ursin (Eds.), Coping and Health. New York: Plenum Press, 1-38.

Peeke, S.C. and Grings, W.W. (1968). Magnitude of UCR as a function of variability in CS-UCS relationship. Journal of Experimental Psychology, 77, 64-69.

Pennebaker, J.W., Burnam, M.A., Schaeffer, M.A. and Harper, D.C. (1977), Lack of control as a determinant of perceived physical symptoms. Joumal of Personality and Social Psychology, $35,167-174$.

Perkins, C.C., Jr., Seyman, R.G., Levis, D.J., and Spence, H., Jr. (1966). Factors affecting preference for signal-shock over shock-signal. Journal of Experimental Psychology, 72, 190196.

Pervin, L.A. (1963). The need to predict and control under conditions of threat. Journal of Personality, 31, 570-587.

Philips, C. (1987). Avoidance Behaviour and its Role in Sustaining Chronic Pain. Behaviour Research and Therapy, 25, 4, 273-279.

Pitman, R.K., Kolk, B.A. van der, Orr, S.P. \& Greenberg, M.S. (1990). Naloxone-reversible analgesic response to combat-related stimuli in posttraumatic stress disorder. Archives of General Psychiatry, 47, 541-544.

Price, K.P. and Geer, J.H. (1972). Predictable and unpredictable aversive events: Evidence for the safety-signal hypothesis. Psychonomic Science, 26, 215-216.

Price, D.D., Barrell, J.J. and Gracely, R.H. (1980). A psychophysiological analysis of experiential factors that selectively influence the affective dimension of pain. Pain, 8, 137-149.

Prins; P.J.M. (1985). Self-speech and self-regulation of high and low-arixious children in the dental situation: an interview study. Behaviour Research and Therapy, 23, 641-650.

Rachman, S. (1977). The conditioning theory of fear-acquisition: A critical examination. Behavlour Research and Therapy, 15, 375-387.

Rachman, S. (1980). Emotional processing. Behaviour Research and Therapy, 18, 51-60.

Rachman, S. (1987). Personal Communication.

Rachman, S. (1988). Panics and their consequences: a review and prospect. In: S. Rachman and J.D. Maser (Eds.), Panic: Psychological Perspectives. Hillsdale, N.J.: Erlbaum, 259-265.

Rachman, S. (1990). Personal Communication.

Rachman, S. (1991). Neoconditioning and the classical theory of fear acquisition. Clinical Psychology Review, in press.

Rachman, S. and Levitt, K. (1985). Panics and their consequences. Behaviour Research and Therapy, 23, 585-600. 
Rachman, S: and Lopatka, K. (1986a). Match and mismatch in the prediction of fear -1. Behaviour Research and Therapy, 24,387-393.

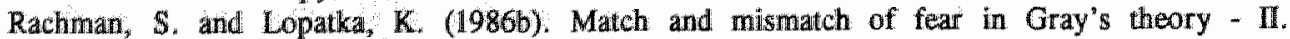
Behaviou Research and Therapy, 24, 395-401.

Rachmiar, S. and Bichard, S. (1988). The overprediction of fear. Clinical Psychology Review, 8, 303-313.

Rachman, s. and Lopatka, K. (1988). Accurate and inaccurate predictions ofpain. Behaviour Research and Therapy, 26, 291-296.

Rachmam, S.J. and Eyrl, $K$ (1989). Predicting and remembering recurrent pain. Behaviour Research and Therapy, 27,621-636.

Rachman, S. and Arnte, A. (1991). The overprediction and underprediction of pain. Cinical Psychology Review, in press.

Read, G.D. (1959). Childbirth without fear." The principles and practice of natural childbirth. New York: Harper.

Reesor, K.A. and Craig, K.P. (1987). Medically incongruent chronic back pain: physical limitations, suffering; and ineffective coping. Pain, 32, 35-45.

Rescorla, R.A. (1978). Some implications of a cognitive perspective on Pavlowian conditioning. In: S.H. Hulse, H.Fowler \& Wh. Honig (Eds.), Cognitive Processes in Animal Behavior. Lawrence Erlbaum Ass., Hillsdale N.J., 15-50.

Rescorla, R.A. and Wagner, A.R. (1972). A theory of Pavlovian conditioning: Variations in the effectiveness of reinforcement and nonreinforcement. In: A.H. Black \& W.F. Prokasy (Eds.), Classical conditioning, Vol.2. Applleton-Century-Crofts, New York, 64-99.

Rhodewalt, F., Strube, M.J. and Wysocki, J. (1988). The Type A behaviour pattern, induced mood, and the illusion of control. European Journal of Personality, 2, 231-237.

Rodin, J., Rennert, K. and Solomon, Sh. (1980). Intrinsic motivation for control: fact or fiction. In: A. Baum and J.E. Singer (Eds.). Advances in Environmental Psychology, Volume 2. Applications of Personal Control. Hillsdale, N.J :: Lawrence Erlbaum.

Romano, J.M. and Turner, J.A. (1985). Chronic pain and depression: Does the evidence support a relationship? Psychological Bulletin, 97, 18-34.

Rose, S.R. \& Orlowski, J. (1983). Review of research on endorphins and learning. Developmental and Behavioral Pediatrics, 4, 131-135.

Rosenbaum, M. (1980). Individual Differences in Self-Control Behaviors and Tolerance of Painful Stimulation. Journal of Abnormal Psychology, 89, 581-590.

Rosenstiel, A.K. and Keefe, F.J. (1983). The use of coping strategies in chronic low back pain patients: relationship to patient characteristics and current adjustment. Pain, 17, 33-44.

Ross, R.T. (1986). Pavlovian second-order conditioned analgesia. Journal of Experimental Psychology: Animal Behawior Processes, 12, 32-39.

Roth, W.T. (1973). Auditory evoked responses to unpredictable stimuli. Psychophysiology, 10 , 125-138.

Rothbaum, F, Weisz, J.R, and Snyder, S.S. (1982). Changing the world and changing the self: A twa-process model on perceived control. Journal of Personality and Social Psychology, 42. 5-37.

Roy, $\mathbb{R}$., Thomas, M., and Matas, M. (1984). Chronic pain and depression, A review. Comprehensive Psychiatry, 25, 96-105.

Sanderman, R., Eysenck, S.B.G. and Arrindell, W.A. (1991). A cross-cultural comparison of personality traits: a study with the Eysenck Personality Questionnaire (EPQ) in Great Britain and the Netherlands. Submitted for publication.

Sanders, A.F. (1961). The influence of noise on two discrimination tasks. Ergonomics, 4, 253258.

Sandman, C.A. (1975). Physiological responses during escape and nonescape from stress in field independent and field dependent subjects. Biological Psychology, 2, 205-216. 
Schell, A.M. and Grings, W.W. (1971). Judgements of UCS intensity and diminution of the unconditioned GSR. Psychophysiology, 8, 427432:

Schmidt, A.J.M. (1985). Performance level of chronic low back pain patients in different treadmill test conditions, Journal of Psychosomatic Research, 29, 639-645.

Schmidt, A.J.M. and Brands, A.M.E.F. (1986). Persistence behavior of chronic low back pain patients in an acute pain situation. Journal Psychosomatic Research, 30, 339-346.

Schmidt, A.J.M. and Arntz, A. (1987). Psychological research and chronic low back pain: a stand-still or breakthrough? Social Sciences and Medicine, 25, 1095-1104.

Seligman, M.E.P. (1968). Chronic fear produced by unpredictable shock. Journal of Comparative and Physiological Psychology, 66, 402-411.

Seligman M.E.P. and Johnston J.C. (1973). A cognitive theory of avoidance learning. In: $F . J$. McGuigan and D.B. Lumsden (Eds.), Contemporary approaches to learning and conditioning. Washington D.C.: Winston and Sons.

Seligman, M.E.P., Maier, S.F., and Solomon, R.L. (1971). Unpredictable and uncontrollable aversive events. In: F.R. Brush (Ed.), Awersive Conditioning and Learning. New York: Academic Press, 1971.

Sherman, R.A., Sherman, C.J. and Parker, L. (1984). Chronic phantom and strump pain among American veterans: results of a survey. Pain, 18, 83-95.

Sherman, R.A., Sherman, C.J. and Bruno, G.M. (1987). Psychological factors influencing chronic phantom limb pain: an analysis of the literature. Pain, 28, 285-295.

Sherrod, D.R., Hage, J.N., Halpern, P.L. and Moore, B.S. (1977). Effects of personal causation and perceived control on responses to an aversive environment: the more control, the better. Journal of Experimental Social Psychology, 13, 14-27.

Shimoff, E., Catania, A.C., and Matthews, B.A. (1981). Uninstructed human responding: sensitivity of low-rate performance to schedule responding. Journal of the Experimental Analysis of Behavior, 36, 207-220.

Siddle, D.A.T. and Heron, P.A. (1975). Stimulus omission and recovery of the electrodermal and digital vasoconstrictive components of the orienting response. Biological Psychology, 3, 277 * 293.

Silver, R.L., Wortman, C.B., and Klos, D.S. (1982). Cognitions, affect and behavior following uncontrollable outcomes: A response to current human helplessness research. Journal of Personality, 50, 480-514.

Sjölund, B., Terenius, L. and Eriksson, M. (1977). Increased cerebrospinal fluid levels of endorphins after electro-acupunture. Acta Physiologica Scandinavica, 100, 382-384.

Skevington, S.M. (1979). Pain and locus of control: a social approach. In: D.J. Oborne, M.M. Gruneberg and J.R. Eisen (Eds.), Research in Psychology and Medicine, Volume 1, 61-69. London: Academic Press.

Skevington, S. (1983a). Social cognitions, personality and chronic pain. Journal of Psychosomatic Research, 27, 421-428.

Skevington, S. (1983b). The changing beliefs, attributions and expectations of early synovitis patients: a longitudinal study of the effects of chronic pain and hospitalization. Pain, supplement 2, S180.

Slade, P.D., Troup, J.D.G., Lethem, J. and Bentley, G. (1983). The fear-avoidance model of exaggerated pain perception - II. Behaviour Research and Therapy, 21, 409-416.

Sokolov, E.N. (1960). Neuronal models and the orienting reflex. In: M.A.B. Brazier (Ed.), The Central Nerwous System and Behaviour. Boston: Josiah Macy Jr." Foundation.

Somsen, R.J.M., Molen, M.W. van der and Orlebeke, J.F. (1983). Phasic heart rate changes in reaction time, shock avoidance, and unavoidable shock tasks: are hypothetical generalizations about different S1-S2 tasks justified? Psychophysiology, 20, 88-94.

Srivastava (1983). Locus of control in migraine patients. Pain. supplement 2, S181.

Staub, E., Turskey, B. and Schwartz, G.E. (1971). Self-control and predictability: their effects on reactions to aversive stimulation. Journal of Personality and Social Psychology. 18, 157-162. 
Watkins, L.R. and Mayer, D.J. (1982). Organization of endogenous opiate and nonopiate pain control systems. Sclence, $216,1185-1192$.

Weichner, G; and Mathews, K.A. (1978). Reported physical symptoms elicited by unpredictable events and the Type A coronary-prone behavior pattern. Journal of Personality and Social Psychology, 36, 1213-1220.

Weisenberg, M. (1987). Psychological intervention for the control of pain. Behavious Research and Therapy. $25,4,301-312$.

Weisenberg, M., Aviram, O., Wolf, Y., and Raphaeli, N. (1984). Relewant and irrelevant anxiety in the reaction to pain. Pan, 20, 371-383.

Weisenberg, M, Wolf, Y., Mittwoch, F., Mikulincur, M. and Aviram, O. (1985). Subject versus experimenter control in the reaction to pain. Pain, 23, 187-200.

Weitkunat, R. and Rosenfeld, I.P. (1986). Pain control by biofeedback of somato sensory evoked potentials. In: Vinck, J. et al. (Eds.), Annual Series of European Research in Behavior Therapy, Volume 1. Topics in Behavioral Medicine, Lisse: Swets and Zeitlinger.

Willer, $J_{1} \mathrm{C}$. and Ernst, M. (1986). Somatovegetative changes in stress-induced analgesia in man: An electrophysiological and pharmacological study. Annals New York Academy of Siciences, $467,256-272$.

Williams, J.M.G., Watts, F.N., MacLeod, C., \& Mathews, A. (1988). Cognitive Psychology and the Emotional Disorders. Chichester: John Wiley.

Willmuth, L., Weaver, L. and Borenstein, J. (1978). Satisfaction with prepared childbirth and locus of control. Journal of Obstetrical and Gynaecological Nursing, 7, 33-37.

Woodrow, K.M., Friedman, G.D. "Siegelaub, A.B. and Collew, M.F. (1972). Pain tolerance. Differences according to age, sex and race. Psychosomaric Medicine, 34, 548-556.

Worthington, (1978). The effects of imagery content, choice of imagery content, and selfverbalization on the self-control of pain. Cognitive Therapy and Research, 2, 3, 225-240.

York,D., Borkovec, T.D., Vasey, M. \& Stern, R. (1987). Effects of worry and somatic anxiety induction on thoughts, emotion and physiological activity. Behawiour Research and Therapy, $25,523-526$.

Zborowski, M. (1969). People in Pain, San Francisco: Jossey-Bass.

Zimbardo, P.G. e.a. (1966). Control of pain motivation by cognitive dissonance. Science, 151, 217-219. 


\section{LIST OF ABBREVIATIONS}

A

$\mathrm{ABV}$

ANOVA

approx.

A1

A2

BAT

BIS

c

C

CAL

CLBP

$\mathrm{cm}$

CNS

$\mathrm{CP}$

CR

CS

D

D

DAS

DC

DNC

E

$\mathrm{Ei}$

EC

EMG

ENC

EPQ

$\mathrm{Fi}$

FPV

$\mathrm{H}$

HA

HA

HR

HRR

HO

I

IASP

IBI

k

L.

LA

LA

M

M

M

$\mathrm{mA}$

Attention directed towards pain condition (sections $4.3 \& 4.4$ )

Amsterdamse Biografische Vragenlijist (De Wilde, 1983)

analysis of variance

aproximately

Actived state of stimulus representation in Wagner's theory

Decay state from A1 to $S$ state in Wagner's theory

Behavioural Approach Test

Behavioural Inhibition System (see Gray, 1975, 1976)

a constant (section 2.3)

correct information ( $=$ control) condition (section 2.3)

calibration phase (refers to measurements during the pretest phase when pain intensity is calibrated)

Chronic Low Back Pain

centimeter

central nervous system

Control (over pain) equated for predictability (section 4.1)

Conditioned Response

Conditioned Stimulus

Difference (discrepancy) between experienced and predicted pain (sections $2.1 \&$ 2.4)

Distraction condition (away from pain)(sections $4.3 \& 4.4$ )

Dental Anxiety Scale (section 2.4)

Depressed mood/Control condition (section 4.2)

Depressed mood/No-Control condition (section 4.2)

Expected (=predicted) pain (section 2.1)

Experienced pain at trial i (sections $2.2 \& 2.3$ )

Elated mood/Control condition (section 4.2)

Electro Myogram (registration of the elctrical activity of a muscle)

Elated mood/No-Control condition (section 4.2)

Eysenck Personality Questionnaire (Sanderman et al., 1991)

subjective Fear at trial i (section 2.3)

Finger Pulse Volume (used to measure vasoconstriction)

High pain prediction or experience (section 2.2)

High dental Anxiety group ('section 2.4)

High Anxiety condition (sections $4.3 \& 4.4$ )

Heart Rate

Heart Rate Response

null hypothesis

Instrumental control over pain (section 4.1)

International Association for the Study of Pain

Inter Beat Interval (heartbeat)

a constant (section 2.3)

Low pain prediction or experience (section 2.2)

Low dental Anxiety group (section 2.4)

Low Anxiety condition (sections $4.3 \& 4.4$ )

mean

mean individual prediction level (section 2.2)

Medium pain prediction or experience (section 2.2)

milliAmpere 


\begin{tabular}{ll} 
MANCOVA & multivariate analysis of covariance \\
MANOVA & muitivariate analysis of variance \\
MD & median \\
min & minute \\
mm & millimeter \\
n.s. & not significant \\
P & Overestimation condition (section 2.3) \\
P & Potential control over pain (section 4.1) \\
P & Pain experience (stection 2.1) \\
Pi & Prediction of pain intensity at trial i (section 2.2 \& 2.3) \\
PC & Personal Computer (section 2.3) \\
PTSD & Post-Traumatic Stress Disorder (section 4.4) \\
P-300 & evoked potential component \\
S & Inaetived state of stimulus representation in Wagner"s theory ('long-term memory") \\
S & Stimulus (section 3.4) \\
S(s) & Subject(s) \\
SA & Self-Administration of a painful stimulation (section 4.1) \\
SC & Skin Conductance \\
SCL & Skin Conductance Level \\
SCR & Skin Conductance Response \\
SD & Standard Deviation \\
Sec & second \\
SPQ & Spider Phobia Questionnaire (Klorman et al., 1974) \\
U & Underestimation condition (section 2.3) \\
UCR & Unconditioned Response \\
UCS & Unconditioned Stimulus \\
VAS & Visual Analogue Scale \\
yr & year \\
\hline
\end{tabular}




\section{ACKNOWLEDGEMENTS}

The author is indebted to Isel van Noppen for her enthusiastic and energetic assistance and her perfect lay-out of the manuscript.

The promotores, Marcel van den Hout and Jack Rachman, gave stimulating and critical comments on research plans and previous versions of research reports and theoretical sections that comprise the present thesis.

Henk Schmidt was chairman of the committee that reviewed the present thesis. Walter Everaerd, Jelle Jolles, Gerjo Kok and Gudrun Sartory were members of the committee.

Peter de Jong assisted during his alternative national services in many experiments and was co-author of various manuscripts. Without his help, and the work of many students, Germie van den Berg, Laura Dreessen, Marleen van Eck; Mariëlle Gelens, Sandra Gransier, Ilse Gubbels, Monique Heijmans, Pieternel Kamp, Richel Lousberg, Birgit Mayer, Anja Meijboom, Henk Nijman and Jaap Oosterlaan; who executed several pilot studies and experiments as part of their study, the present thesis would simply not exist.

Harald Merckelbach, Rutger Lulofs, Margo van der Molen and Co Orlebeke gave valuable psychophysiological advice.

Marcel van den Hout, Anita Jansen, Peter de Jong, Harald Merckelbach, Ton Schmidt, Eric Schouten and most of above mentioned students were co-authors of various papers reprinted in the present dissertation. Andrew Steptoe and Ad Appels reviewed the manuscript which appeared as chapter 7 in their book, and which is reprinted as section 4.1 in the present dissertation.

Angela Verweij gave general secretarial assistance for the research project Experimental Psychopathology. Riet Gierlings, Dorien Wolfs and Gerry van Wunnik gave indispensable assistance during the experiments. Jan Talmon made a computer-program for transforming heart beat intervals into beats per minute. Theo van Aerts perfected this program, and did also other valuable computer-programming. Jan van Houtem and Eric Schouten executed some complex computations (sections $2.1 \& 2.2$ ). Dr. van Grunswen provided us with a Dutch translation of the Dental Anxiety Scale. Math Frederikson sent us a version of the Spider Phobia Questionnaire. Prof. van der Lugt and mr. Cauberg of the Academic Hospital Maastricht gave invaluable help in finding a suitable pain stimulator. Jan Geilen, Jeroen Hameleers, Maurice Huinck, Hans Koper and Eugene Wintjens of the Instrumental Department did the necessary electronic work. Olga Sollet assisted in the production of the figures.

The dentists, R.H.J. Klinkers, M. Ruypers and Th. Kluskens, their assistants, G. Gilissen, T. Huijnen and M. Bessems, and their patients are acknowledged for their help in the dental anxiety and pain study.

Pavlos Anastasiades, Omer van den Berg, Gillian Butler, David Clark, Paul Eelen, Melanie Fennell, Gerry Kent, Clare Philips, Paul Salkovskis, and numerous (anonymous) others gave valuable comments.

Marijke van der Horst and Alan Ralston proof-read and corrected the text.

Last, but not least, the subjects that participated in the various experiments are acknowledged. 



\section{CURRICULUM VITAE}

De auteur werd geboren op 30 november 1956 te Utrecht. Hij behaalde in 1975 het eindexamen gymnasium B. Van 1975 tot 1980 studeerde hij natuurkunde aan de Rijksuniversiteit Groningen met als bijvak filosofie. Hij behaalde in 1979 het kandidaatsexamen natuurkunde. In 1980 beëindigde hij de studie natuurkunde om psychologie aan dezelfde universiteit te gaan studeren. In mei 1985 behaalde hij het doctoraal examen psychologie, met als afstudeerrichting klinische psychologie. In 1985 en 1986 verrichtte hij als wetenschappelijk assistent aan de Rijksuniversiteit Groningen onderzoek naar adaptief psychologisch testen. Sinds april 1986 is hij als universitair docent verbonden aan de Rijksuniversiteit Limburg, vakgroep Medische Psychologie, alwaar hij naast het geven van onderwijs binnen de faculteit Gezondheidswetenschappen, onderzoek verricht naar pijn. Daarnaast heeft hij organiserende en psychotherapeutische taken in de Geacademiseerde Sectie Gedragstherapie van de RIAGG Maastricht. In dat kader volgt hij ook de RINOopleiding tot basis-psychotherapeut en verricht hij onderzoek naar angst- en persoonlijkheidsstoomissen en naar hun behandeling. 